\title{
DESARROLLO DE UN MODELO ANALÍTICO DE PREDICCIÓN DE FUERZAS EN EL MICROFRESADO APLICADO A MICROFABRICACIÓN
}

Autor: Pablo Rodríguez Mateos

Director: Julio Labarga Ordóñez 


\section{ÍNDICE}

1. OBJETIVO GENERAL DEL ESTUDIO

1.1 Importancia actual de la Microfabricación

1.2 Alcance del Modelo

1.3 Materiales y equipos utilizados

2. ESTADO ACTUAL DE LA TECNOLOGÍA DEL MICROFRESADO

2.1 Visión global de la investigación relacionada

2.2 Microtecnologías en general

2.3 Microfresado

2.4 Fresado convencional

2.4.1 Estudios iniciales

2.4.2 Estudios realizados sobre el proceso de fresado

2.4.3 Análisis de los parámetros del fresado

2.4.4 Modelos de fuerzas en el fresado

2.4.5 Investigación en microfresado

2.4.6 Análisis dinámico

2.4.7 Control adaptativo

3. ANÁLISIS DE LOS FACTORES DE INFLUENCIA EN EL PROCESO

3.1 Fresado en condiciones ideales

3.1.1 Expresiones para fresado en oposición

3.1.2 Expresiones para fresado en concordancia

3.2 Influencia de la desviación excéntrica de la herramienta

3.3 Corte asimétrico

3.4 Influencia de la deflexión de herramienta

3.4.1 Formulación del modelo

3.4.2 Cálculo de constantes

3.4.2.1 Fresas Sandvik

3.4.2.2 Fresas DIXI

4. DESARROLLO DEL MODELO

4.1 Fresado en oposición 
4.1.1 Procedimiento de cálculo del espesor de viruta en condiciones de corte simétrico

4.1.2 Efecto tamaño

4.1.3 Resultados experimentales

4.2 Microfresado en concordancia

4.2.1 Procedimiento de cálculo del espesor de viruta en condiciones de corte simétrico

4.2.2 Efecto tamaño

4.2.3 Resultados experimentales

5. PROGRAMACIÓN DE LA RESOLUCIÓN DEL MODELO

5.1 Cómputo de los diferentes tipos de fuerzas

5.1.1 Fresado en oposición

5.1.2 Fresado en concordancia

6. DETERMINACIÓN DE LOS PARÁMETROS DEL MODELO

6.1 Ensayo de fuerza constante

6.1.1 Fresado en oposición

6.1.2 Fresado en concordancia

6.2 Ensayo de fuerza variable

6.2.1 Fresado en oposición

6.2.2 Fresado en concordancia

6.3 Medición de la desviación excéntrica de herramienta

6.4 Medición del radio de filo de la herramienta

6.5 Medición de la rigidez de la herramienta

7. ANÁLISIS DE LAS VIBRACIONES DEL SISTEMA

7.1 Determinación de la frecuencia natural del sistema

7.2 Trabajo en condiciones de retemblado

7.3 Trabajo en condiciones de no retemblado

7.4 Filtrado de la señal de fuerzas

7.4.1 Filtro paso bajo

7.4.2 Métodos alternativos de suavizado 
8. APLICACIONES DEL MODELO

8.1 Monitorización del proceso

8.2 Control adaptativo

8.2.1 Descripción del sistema

8.2.2 Función de Transferencia discreta del sistema de control

9. REFERENCIAS BIBLIOGRÁFICAS 


\section{OBJETIVO GENERAL DEL ESTUDIO}

\subsection{Introducción}

El objetivo de la investigación realizada es obtener un modelo de fuerzas para las diversas operaciones de microfresado que permita realizar predicciones de los valores de las fuerzas del proceso de manera automática mediante su programación en un ordenador, de tal manera que pueda ser útil en sistemas de monitorización y control adaptativo.

\subsection{Importancia actual de la Microfabricación}

En la actualidad la miniaturización de dispositivos requiere la producción de componentes electromecánicos en los que se precisan geometrías en el rango de unos pocos micrómetros a varios cientos de micrómetros en campos como la óptica, la electrónica y la medicina. Algunas aplicaciones específicas son las células de energía, los microrreactores químicos que requieren microbombas, micromoldes y muchas más. La figura 1.1 muestra algunos ejemplos de piezas y geometrías obtenidas por micromecanizado. Estas aplicaciones requieren tolerancias muy estrechas así como ciertas especificaciones estructurales y funcionales. Muchas de estas piezas deben ser metálicas, para lo cual el microfresado es uno de los métodos más adecuados para fabricarlas [1].

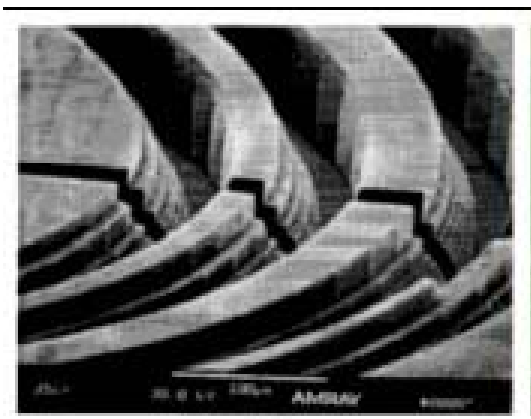

(a)

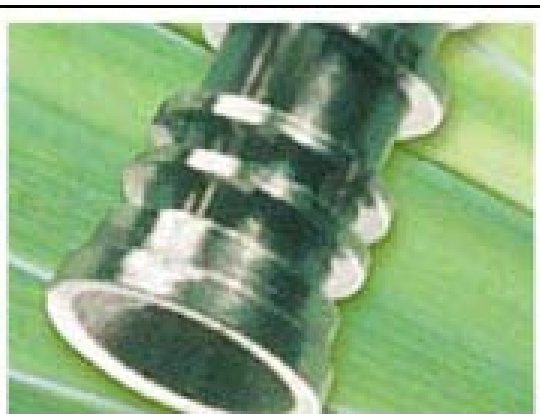

(b)

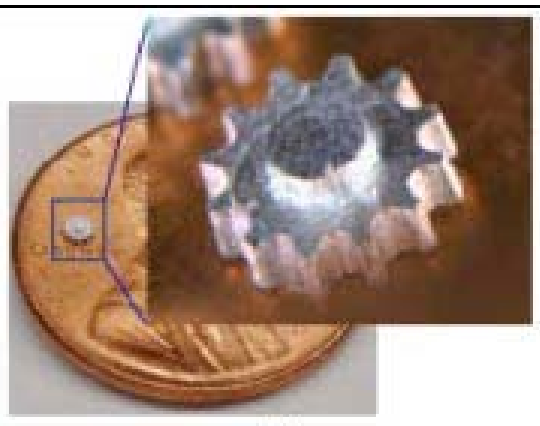

(c)

Figura 1.1. a) Cavidades microfresadas con paredes escalonadas, b) componente de un dispositivo neurovascular, c) microengranaje 
En el caso del microfresado hay factores que no se consideran en el fresado convencional, como la desviación excéntrica de la herramienta, la deflexión de la herramienta o el efecto tamaño, ya que su influencia no es significativa a escalas macro, mientras que a pequeñas escalas sí son importantes, dado que cualquier pequeña desviación de la posición de herramienta respecto a su posición teórica provoca variaciones significativas en su trayectoria y por tanto en las fuerzas del proceso.

\subsection{Importancia de la predicción de fuerzas en el Microfresado}

Como se ha indicado, esta Tesis Doctoral persigue el estudio de las fuerzas del proceso con el objeto de desarrollar un modelo predictivo de uso en la industria del microfresado.

El modelo analítico de fuerzas constituye una herramienta de crucial importancia para el desarrollo de sistemas de control adaptativo, en los que se regula el proceso de acuerdo con sus condiciones instantáneas. Asimismo, puede ser evaluado el desgaste de herramienta y detectada la rotura, ya que ambos fenómenos tendrán un reflejo en las fuerzas medidas del proceso. Todo ello tiene gran importancia en la práctica industrial del microfresado, en la que el proceso presenta condiciones de trabajo adversas que conviene controlar estrechamente para conseguir una adecuada productividad.

Mediante la determinación de los parámetros del modelo se podrá confirmar la importancia de los distintos factores de influencia en el proceso de microfresado con datos cuantitativos, que justifican su consideración en el modelo.

Dado que el microfresado es un proceso con cierta complejidad será conveniente fijar algunas pautas para aplicar en la práctica. Gracias al modelo de fuerzas se podrán deducir algunas condiciones geométricas en las que la herramienta trabaja mejor debido a la estabilidad de las fuerzas, a la ausencia de cambios bruscos y a la eliminación de picos de alto valor. Asimismo, el estudio de las medidas experimentales de las fuerzas permitirá establecer un rango de velocidades de corte para cada material en el que el proceso es más seguro. Lo mismo sucederá con otras condiciones de corte como la 
profundidad axial de corte y el avance por diente. Esto permitirá fijar una serie de recomendaciones que hagan más seguro el proceso de microfresado.

Se puede indicar la utilidad del modelo de fuerzas mediante los siguientes puntos:

A. El modelo de fuerzas permitirá deducir algunas magnitudes del proceso de gran importancia para su caracterización, tales como el par, la potencia, la energía y el calor generado en el proceso. La estimación de la temperatura en las distintas zonas de corte puede servir de guía para un ulterior desarrollo del modelo en el que se incluyan los efectos térmicos, que pueden tener a su vez una influencia importante en la precisión de la pieza y sobre todo en el estudio de la microestructura de la superficie mecanizada.

B. El modelo analítico de fuerzas permitirá por otra parte determinar la desviación de la herramienta respecto a su posición teórica en cada momento del proceso, lo cual se podrá utilizar para estudiar la precisión de la superficie mecanizada y evaluar, por tanto, la validez del proceso para la obtención de piezas con determinadas tolerancias.

C. La predicción de fuerzas de corte a partir de los parámetros del proceso permite además llevar a cabo un control eficaz del proceso, de manera que se pueda actuar rápidamente ante situaciones de falta de estabilidad, pérdidas de homogeneidad en el material y errores de programación.

En resumen, se puede decir que esta Tesis Doctoral se propone el objetivo de analizar el proceso de microfresado en orden a comprender mejor las variables que intervienen, identificar los factores de influencia y controlar mejor el proceso.

Por ello, en primer lugar, se pretende realizar un análisis exhaustivo de los factores que intervienen en el proceso a partir de estudios ya realizados para fresado convencional y extendiendo la investigación al proceso específico del microfresado. Los principales factores de influencia en las fuerzas de corte que se analizarán en esta investigación serán los siguientes: 
- Efecto Tamaño

- Trayectoria trocoidal del filo

- Desviación excéntrica de la herramienta

- Deflexión de herramienta

- Desviaciones dinámicas de la herramienta

Se realizará una caracterización cuantitativa de estos factores, analizando las variables físicas y geométricas que intervienen en cada uno de ellos, de tal manera que pueda hacerse una evaluación de su importancia para el microfresado y por tanto de la conveniencia o no de incluirlos en un modelo analítico para el estudio del proceso.

Con los resultados de dicho análisis se podrán integrar los factores con influencia significativa en el proceso para desarrollar un modelo analítico de fuerzas que proporcione predicciones fiables para el proceso real.

El modelo utilizará algunos parámetros que caracterizan los distintos factores de influencia que se han considerado para su desarrollo. Estos parámetros se han obtenido mediante medición directa en algunos casos, como la desviación excéntrica y la rigidez de la herramienta, y en otros se han calculado analíticamente, como el momento de inercia o el momento resistente de las herramientas. Eventualmente se han tomado algunos datos experimentales que aportan estudios previos, como la relación entre el espesor mínimo de viruta y el radio de redondeo del filo.

\subsection{Alcance del Modelo}

El modelo se desarrollará para fresas de dos dientes con forma helicoidal. Estas fresas son las más comúnmente usadas en las operaciones de microfresado, debido a la dificultad que existe en la fabricación de fresas de reducidas dimensiones con más de dos dientes. Por otro lado, partiendo de un modelo para fresas de dos dientes es relativamente sencilla la generalización del procedimiento de modelado para fresas con más dientes, dado que consistiría únicamente en añadir a la señal de las fuerzas nuevos 
periodos angulares de corte, mediante la estrategia de programación usada en el modelo original.

La predicción de fuerzas se ha limitado a las direcciones horizontales $x$ e $y$. La razón de esta simplificación es que la fuerza horizontal es la que más relevancia tiene en la deflexión de herramienta y por tanto la que puede provocar la rotura de la herramienta. Por otro lado, el control adaptativo del proceso puede tener lugar de manera adecuada mediante la monitorización de las fuerzas en las direcciones horizontales, ya que con ellas se puede llevar a cabo el sistema de estimación automática de parámetros y por lo tanto caracterizar perfectamente el proceso y sus condiciones.

\subsection{Material y equipos utilizados}

Los ensayos de mecanizado fueron realizados en una Máquina KERN de Microfresado y Microtaladrado, que dispone de un husillo de hasta 160.000 r.p.m. de velocidad de giro y un Control Numérico Computerizado con una resolución de posicionamiento de $0.1 \mu \mathrm{m}$. La Máquina de Microfresado se muestra en la figura 1.2.

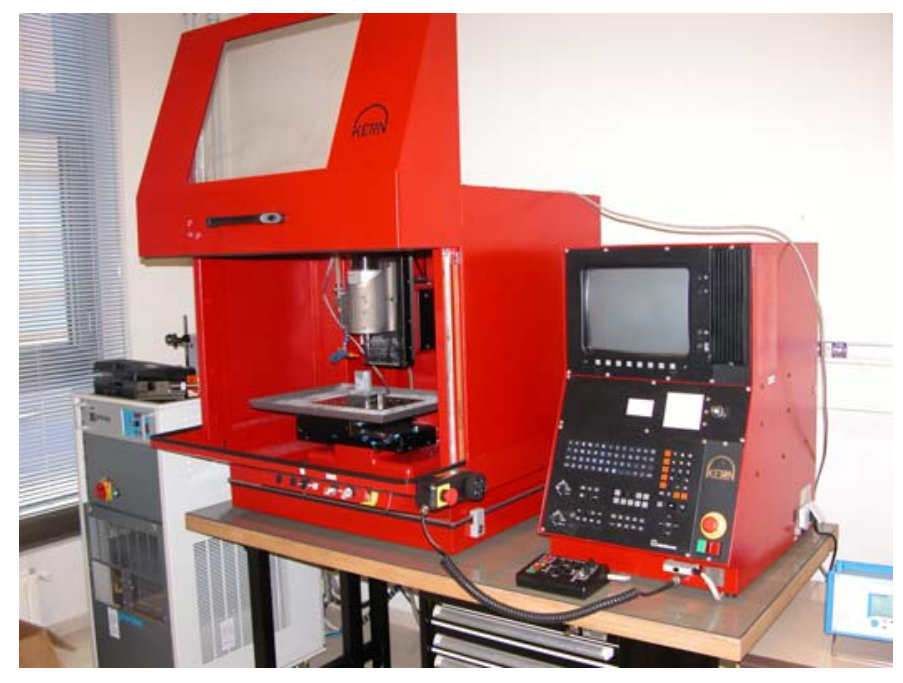

Figura 1.2. Máquina de Microfresado

Para validar el modelo se realizará un contraste de los resultados del modelo con las mediciones experimentales para distintas condiciones de corte y tipos de fresado. De esta manera se podrá realizar un estudio del campo de validez del modelo e identificar 
sus límites. En la tabla 1.1 se indican las principales variables tenidas en cuenta en la experimentación y los valores considerados.

\begin{tabular}{l|cc}
\hline Variables del proceso & \multicolumn{2}{|c}{ Valores considerados } \\
\hline Tipo de fresado & En oposición & En concordancia \\
Material & Aluminio & Acero \\
Velocidad de corte & Baja & Media \\
\hline Tipo de herramienta & DIXI & Sandvik \\
\hline
\end{tabular}

Tabla 1.1. Valores de las distintas variables utilizados en la experimentación

Los ensayos fueron realizados en Aluminio 7075, así como en acero F-1110 y F-1540, con el objeto de caracterizar el proceso en los materiales más habituales usados en la industria. Se llevaron a cabo operaciones de fresado en oposición y en concordancia. El tamaño de las fresas usadas varía entre 0.2 y $1 \mathrm{~mm}$ de diámetro.

Se utilizaron fresas de Carburo de Wolframio sin recubrimiento, de dos marcas: DIXI y Sandvik. Cada tipo tiene distinto valor del radio de filo, lo cual permitió observar la influencia de la variación de esta magnitud en los datos experimentales de las fuerzas, como se indicará más adelante.

Las medidas de las fuerzas de corte fueron tomadas con un dinamómetro Kistler 9256C2. Este dispositivo utiliza el efecto piezoeléctrico de los cristales de cuarzo de que está compuesto y envía la señal a un amplificador, que a su vez la envía al ordenador mediante una tarjeta analógico-digital IOTech DaqBoard 2000. Desde el ordenador se captura la señal mediante el software DaisyLab, que permite la visualización y el tratamiento de los datos.

Se llevó a cabo mecanizado en seco, ya que, según se indicará en el capítulo 2, los fluidos de corte presentan numerosos inconvenientes y las ventajas que se obtienen de 
su utilización, consistentes en la disminución de las fuerzas de corte, no justifican el recurso a dichos fluidos.

Los ensayos se hicieron fuera de la situación de retemblado. Aun así, se empleó un filtro paso-bajo de entre 2000 y $3000 \mathrm{~Hz}$ para eliminar la señal procedente de la frecuencia natural del sistema, que tiene lugar entre 4000 y $5000 \mathrm{~Hz}$. Los datos se tomaron a una frecuencia de muestreo de $100 \mathrm{kHz}$, de tal manera que se pudiese apreciar con suficiente nitidez las señales a la frecuencia de trabajo. 


\section{ESTADO ACTUAL DE LA TECNOLOGÍA DE MICROFRESADO}

\subsection{Introducción}

El Microfresado es un proceso que se enmarca dentro del campo de las Microtecnologías, siendo el fin de estas últimas la fabricación de microcomponentes a partir de diversos procesos, como el mecanizado convencional, la electroerosión, el moldeo por inyección, el haz de iones, el mecanizado láser, o procesos más específicos de la escala microscópica como la litografía o la deposición química de vapor. También comprende el estudio del amplio campo del microensamblaje, una tecnología necesaria para la obtención de productos terminados en grandes o pequeños volúmenes. Por su parte, el Microfresado es una tecnología fundamentada en el fresado convencional y, por tanto, en las técnicas de mecanizado. Por tanto, para realizar un recorrido en el estado actual del Microfresado habrá que estudiar primero su contexto dentro de las Microtecnologías y posteriormente prestar atención al conocimiento en el campo del fresado convencional, así como de los modelos analíticos de fuerza, que se aplicarán íntegramente al campo de estudio del presente trabajo.

En el proceso de Microfresado confluyen varias líneas de estudio que aportan conocimiento en dicha tecnología y de las cuales se toman los resultados y conclusiones para su aplicación inicial a la presente investigación. Todo ello se une a los estudios realizados en el terreno específico del Microfresado que analizan aspectos concretos del proceso. Las líneas más importantes que se han tenido en cuenta en la presente revisión se señalan a continuación:

- Microfabricación

- Microfresado

- Fresado convencional

o Modelos de fuerzas

o Estudios de desgaste y vida de herramienta

o Precisión y calidad superficial en la pieza 


\section{o Análisis dinámico \\ o Control adaptativo}

El Microfresado es un proceso al que se aplican todos los resultados obtenidos en el estudio del mecanizado, además de otros aspectos específicos que surgen por estar trabajando a pequeñas dimensiones, que se detallarán en los estudios sobre Micromecanizado en general. En este campo se han estudiado con cierta extensión varios procesos como Microtorneado, Microtaladrado, Microrrectificado o el Micromecanizado láser. Asimismo, existen otras técnicas como las litográficas, la de deposición de vapor química o física o la Microelectroerosión, que completan el enorme campo de la Microfabricación. El estudio comparado de todos ellos ayudará a situar la técnica del Microfresado en su contexto, así como a determinar el campo de aplicación del proceso y por tanto los aspectos en que es conveniente resaltar la comprensión de esta tecnología.

Por otro lado, la investigación en Fresado convencional es de aplicación necesaria al proceso del Microfresado, ya que conceptualmente es la misma técnica. Son de interés, por tanto, todos los estudios que aborden las características generales del proceso, los materiales de herramientas utilizadas, la microestructura de la pieza mecanizada, las vibraciones y el retemblado y las aplicaciones y limitaciones del proceso

Dado que los errores de posición y velocidad de la herramienta son de especial relevancia en el proceso de microfresado debido a sus reducidas dimensiones, se prestará atención a todos los trabajos llevados a cabo en este terreno tanto a grandes como a pequeñas escalas, revelando así factores de influencia en el proceso a los que se prestará atención en el desarrollo del modelo.

El modelo desarrollado en este trabajo se considera de gran utilidad para el control adaptativo del proceso y la evaluación de la precisión de la superficie mecanizada. Por tanto, en la investigación es necesario tener en cuenta el desarrollo de las técnicas actuales de control adaptativo y monitorización de variables en el proceso, en orden a desarrollar un modelo en línea con su futura aplicación en este campo. Asimismo, convendrá revisar también los estudios existentes sobre precisión en el proceso para destacar las aportaciones que representa el uso del modelo en este terreno. 
El desgaste es una variable de influencia en el mecanizado en general y por tanto, será necesario tenerla en cuenta también en el microfresado, dado que afectará significativamente a las fuerzas de corte y a la precisión de las piezas mecanizadas de una manera mayor que en el mecanizado convencional, afectando también a la vida de herramienta.

También se revisará la bibliografía existente en todos estos terrenos, destacando las contribuciones más importantes a la comprensión y caracterización del microfresado, dejando para una sección posterior la revisión de los modelos de fuerzas en mecanizado, dado que merecen una especial atención por su especial relación con el presente trabajo.

\subsection{Microtecnologías en general}

\subsubsection{Procesos de Microfabricación}

En los últimos años las microtecnologías han experimentado un fuerte avance fuera del campo de la microelectrónica, ámbito en el que estaba confinada la fabricación de elementos a pequeña escala.

Actualmente existen varios procesos de micromecanizado en desarrollo. Los procesos llevan asociados unos parámetros que dan idea de las ventajas y desventajas de cada uno. Algunos parámetros que se utilizan son la Unidad de Eliminado de material (UR) [2], precisión, volumen total de material eliminado y tipo de forma que se puede mecanizar.

Se describen a continuación los procesos más importantes de micromecanizado de piezas.

- Micromecanizado por fusión o vaporización del material:

En este proceso se elimina material mediante fusión y evacuación, que se consigue aportando una gran cantidad de calor en un área muy pequeña de la pieza. Esta 
vaporización se puede conseguir por varios métodos, entre ellos el de la Electroerosión (EDM), que da lugar al Micromecanizado por Electroerosión.

Con alguno de estos métodos se producen cambios microestructurales en las proximidades del material eliminado, sin embargo son dimensionalmente muy precisos.

- Micromecanizado por ablación láser

- Moldeado y deformación plástica

- Litografía

\subsubsection{Resultados obtenidos}

Mediante estas técnicas se han podido fabricar piezas de muy pequeño tamaño con gran nivel de resolución.

Se han llegado a fabricar microbombas de engranajes con rotores de $596 \mu \mathrm{m}$ de diámetro, de gran utilidad en medicina [3]. Con la técnica de Micromecanizado por Electroerosión con hilo se han conseguido fabricar agujas de $4.5 \mu \mathrm{m}$ de diámetro. Otros ejemplos de microfabricación son microturbinas, microinductores [4], herramientas para sistemas de fibra óptica [5], etc.

Estas microtecnologías han obtenido ya unos resultados muy prometedores, pero requieren un desarrollo mayor y una adaptación a la fabricación a nivel industrial.

La tabla 2.1 resume la utilización y características de las microtecnologías más frecuentes. 


\begin{tabular}{|c|c|c|c|c|}
\hline & $\begin{array}{c}\text { Microfresado } \\
\text { mecánico }\end{array}$ & $\begin{array}{c}\text { Electro } \\
\text { descarga }\end{array}$ & $\begin{array}{c}\text { Microfresado } \\
\text { láser }\end{array}$ & Ultrasonidos \\
\hline $\begin{array}{l}\text { Tamaño } \\
\text { objeto }\end{array}$ & $20 \mu \mathrm{m}$ & $5 \mu \mathrm{m}$ & $10 \mu \mathrm{m}$ & $5 \mu \mathrm{m}$ \\
\hline $\begin{array}{l}\text { Tamaño de } \\
\text { la partícula } \\
\text { retirada }\end{array}$ & $1 \mathrm{~nm}$ & $0.01 \mathrm{~nm}$ & $0.5 \mathrm{~nm}$ & $0.5 \mu \mathrm{m}$ \\
\hline Error & $0.04-0.5 \mu \mathrm{m}$ & $0.2 \mu \mathrm{m}$ & $0.2 \mu \mathrm{m}$ & $0.05 \mu \mathrm{m}$ \\
\hline Proceso & Fresado & $\begin{array}{l}\text { Electroerosión } \\
\text { por hilo }\end{array}$ & Excimer Láser & $\begin{array}{l}\text { Vibración de } \\
\text { herramienta } \\
\text { abrasiva }\end{array}$ \\
\hline $\begin{array}{c}\text { Geometría } \\
\text { obtenida }\end{array}$ & Cavidades & $\begin{array}{c}\text { Formas cóncavas } \\
\text { y convexas }\end{array}$ & $\begin{array}{l}\text { Duplicación } \\
\text { superficies }\end{array}$ & Cavidades \\
\hline Ventaja & $\begin{array}{l}\text { Equipamiento } \\
\text { conocido y de } \\
\text { fácil manejo }\end{array}$ & $\begin{array}{c}\text { Fuerzas de } \\
\text { mecanizado muy } \\
\text { pequeñas }\end{array}$ & $\begin{array}{l}\text { No influencia } \\
\text { térmica } \\
\text { alrededor }\end{array}$ & $\begin{array}{l}\text { Capacidad de } \\
\text { mecanizar } \\
\text { materiales } \\
\text { frágiles }\end{array}$ \\
\hline $\begin{array}{c}\text { Materiales } \\
\text { mecanizables }\end{array}$ & $\begin{array}{l}\text { Metales, } \\
\text { plásticos, } \\
\text { compuestos }\end{array}$ & $\begin{array}{l}\text { Metales y } \\
\text { aleaciones }\end{array}$ & Casi todos & $\begin{array}{l}\text { Vidrio, } \\
\text { Cerámicos, } \\
\text { Grafito }\end{array}$ \\
\hline
\end{tabular}

Tabla 2.1. Resumen de algunas de las Microtecnologías más frecuentes

\subsection{Microfresado}

La investigación en microfresado es muy amplia y se ha venido desarrollando desde finales de los años 90, con numerosas contribuciones en distintos aspectos de esta tecnología. Sin embargo, la tecnología del microfresado es un desarrollo natural del fresado convencional, ya que conceptualmente es el mismo proceso, aunque influyan distintos factores y se lleve a cabo en distintas condiciones. En el campo específico de modelos de fuerzas de microfresado se ha llevado a cabo un desarrollo ulterior a partir de los modelos de fuerzas de fresado convencional añadiendo la consideración de factores de influencia específicos de las pequeñas escalas.

Un aspecto muy importante del fresado es el comportamiento dinámico del conjunto herramienta-pieza, que da lugar a vibraciones en la posición de la pieza y por tanto en 
las fuerzas de corte. Este aspecto es más importante aún a pequeñas escalas, ya que cualquier pequeña variación de posición implica una distorsión relativa muy importante en las fuerzas estáticas. Este estudio permite evitar además el fenómeno del retemblado, en el que la amplitud de las vibraciones se dispara, provocando una gran rugosidad en la pieza y un alto riesgo de rotura de la herramienta. Se ha de estudiar, por tanto, este fenómeno para caracterizar correctamente el modelado de las fuerzas.

Por otro lado, se han de tener en cuenta las aplicaciones del modelado de fuerzas, como son la estimación previa de condiciones de corte, la monitorización y el control adaptativo y la predicción de rotura de herramienta.

Todo ello ha de ser analizado mediante la consideración de la investigación existente en esos terrenos. En los apartados siguientes se realizará una revisión de todos los trabajos realizados que contribuyen al estudio de los diferentes aspectos del microfresado: el fresado convencional, los modelos de fuerzas en el fresado, el microfresado propiamente dicho, el análisis dinámico del proceso y el control adaptativo y otras aplicaciones.

\subsection{Fresado convencional}

El fresado es una de las operaciones de fabricación más usada en la industria para el conformado de metales y otros materiales. Este proceso ha sido utilizado como técnica muy versátil para el conformado de piezas de gran variedad de geometrías tales como perfilados, vaciados, ranurados, producción de engranajes, etc. [6]. El proceso ha sido llevado a cabo en multitud de materiales, tales como metales de todo tipo, madera y plásticos. Por tanto, estamos ante un proceso muy ventajoso en cuanto a las posibilidades que ofrece y su capacidad de automatización [7].

Dada la importancia del desarrollo de esta técnica se han realizado a lo largo de los años muchos avances en distintos aspectos, tales como mejora de la forma y materiales de la herramienta [8], estudio de las vibraciones del sistema [9], análisis de la integridad superficial de la pieza mecanizada [10], acabado superficial de la pieza [11], etc. 
También se han llevado a cabo estudios de importancia en cuanto al diseño de las piezas con vistas a su mecanizado [12] y de las máquinas usadas para el proceso [13].

El modelado y la simulación de los procesos de fresado son de gran utilidad en el diseño del proceso, la maquinaria y el análisis del fenómeno. También se emplea para determinar la precisión dimensional del proceso. Una utilidad importante que tienen los modelos de fuerzas es la determinación de las condiciones de corte óptimas para una buena productividad, calidad de la pieza y vida de herramienta.

\subsubsection{Estudios iniciales}

Los procesos de mecanizado se han investigado extensamente desde principios del siglo $\mathrm{XX}$ en campos tan diversos como desgaste y vida de herramienta, geometría del filo, materiales de herramienta, condiciones de corte, etc.

El fresado como objeto específico de estudio surge en el año 1945 con el trabajo de M.E. Martelotti [14], en el que se analiza el recorrido de la herramienta y el fenómeno del filo de aportación. Posteriormente, en 1961 surgen los trabajos de Koenigsberger y Sabberwal [15,16], que caracterizan matemáticamente las fuerzas y proporcionan nomogramas en los que se relacionan las condiciones de corte con la fuerza media del proceso.

Koenigsberger y Sabberwal analizan por separado el fresado frontal y el cilíndrico. En su investigación se determina la posición óptima de la herramienta en el fresado frontal para que las fuerzas sean lo más regulares posible. Se desarrollan expresiones generales para cualquier número de filos, así como nomogramas y la relación entre fuerza máxima y fuerza media y se establece una relación entre la presión específica de corte y el espesor de viruta.

El número máximo de filos que cortan simultáneamente se establece como:

$$
m=\frac{\phi_{s}+\phi_{b}}{\phi_{t}}
$$


Donde $\phi_{s}$ es la anchura angular de corte, es decir la diferencia entre el ángulo de salida y entrada y $\phi_{b}$ es el ángulo que existe entre la extremo inferior del filo y el punto del filo a la altura de la profundidad axial de corte y $\phi_{t}$ es la distancia angular entre filos.

\subsubsection{Estudios realizados sobre el proceso de fresado}

A partir de 1970 surgen investigadores como Armarego, Tlusty y Kline, que realizan aportaciones importantes que permitirán un desarrollo posterior muy extenso en el terreno de los modelos analíticos de fuerzas del fresado.

Desde entonces se han desarrollado numerosos estudios para analizar la geometría de la herramienta y la trayectoria de sus filos. Este análisis se utiliza para calcular el espesor de viruta en cada punto del filo y en cada posición de la herramienta, de tal manera que se pueda determinar la fuerza que ejerce la herramienta con la expresión analítica correspondiente.

También se ha investigado sobre la relación entre la fuerza y el área de viruta eliminada. Este estudio ha adquirido en ocasiones un carácter más teórico, partiendo del análisis general de la geometría y la mecánica del corte ortogonal y realizando la transformación correspondiente al corte específico del fresado. Para utilizar este tipo de modelos se necesitan bases de datos de parámetros del corte para una gran variedad de materiales de pieza y herramienta. Todo ello ha sido convenientemente desarrollado y aplicado al fresado.

Estos estudios se complementan con muchos otros modelos propuestos para la predicción de fuerzas en el proceso, como se indicará más adelante.

Se ha analizado también la rugosidad de la superficie mecanizada a partir de la geometría del proceso con el propósito de determinar las condiciones de corte más adecuadas a la calidad requerida de la pieza.

El uso de fluidos de corte ha sido también objeto de investigación en el fresado, así como en los demás procesos de mecanizado [17]. Se ha concluido que en la medida de 
lo posible ha de evitarse el uso de dichos fluidos, ya que presentan numerosos inconvenientes, como el perjuicio al medio ambiente, el aumento del coste del proceso y el posible daño a la salud de los operarios. Algunos autores indican que no siempre es posible el mecanizado en seco, dado que no se pueden evitar problemas como la adhesión de la viruta a la herramienta, el excesivo desgaste de herramienta o la dilatación incontrolada de la pieza [18].

Otra aportación importante es el estudio de las vibraciones en el proceso y el comportamiento dinámico de la herramienta y la pieza. Las vibraciones tienen una enorme influencia en la precisión de la pieza mecanizada, según se ha demostrado mediante simulación y experimentación [19]. El fenómeno de las vibraciones autoexcitadas afecta a la rugosidad y a la precisión de la pieza y es conocido como retemblado. Para caracterizarlo es necesario determinar los parámetros de comportamiento dinámico del conjunto herramienta-pieza, según se describe en el apartado 2.4.6.

Es importante mencionar también los estudios realizados en el campo de la monitorización del proceso, ya que este sistema permite realizar de una manera mucho más eficiente el proceso. Un proceso de mecanizado implica interacciones complejas entre máquinas, herramientas, piezas, sistemas de medida, sistemas de movimiento de piezas, etc., por lo que una monitorización de las variables de influencia en el proceso permitirá llevar a cabo una producción eficiente, con mínimo coste y control de las necesidades de mantenimiento. Existen trabajos en los que se describen las tendencias actuales y el papel de los sistemas de monitorización [20]. Ya los primeros trabajos demostraron que la monitorización del proceso es fundamental para una producción económica. Los variables más importantes a monitorizar de cara a la fiabilidad y la calidad del proceso son el desgaste de herramienta y las condiciones de rotura de herramienta [21]. Algunas de las maneras típicas de aplicar la monitorización son la medición o identificación de la interacción entre el proceso y la estructura de la máquina, de forma similar a como se estudia el comportamiento dinámico del sistema máquina-herramienta-pieza. 
Algunos autores, como Tönshoff, han realizado estudios de la evolución de los sistemas de monitorización en la fabricación [22], que se pueden resumir en la tabla 2.2, que indica la fase de desarrollo e implementación de cada tipo de monitorización.

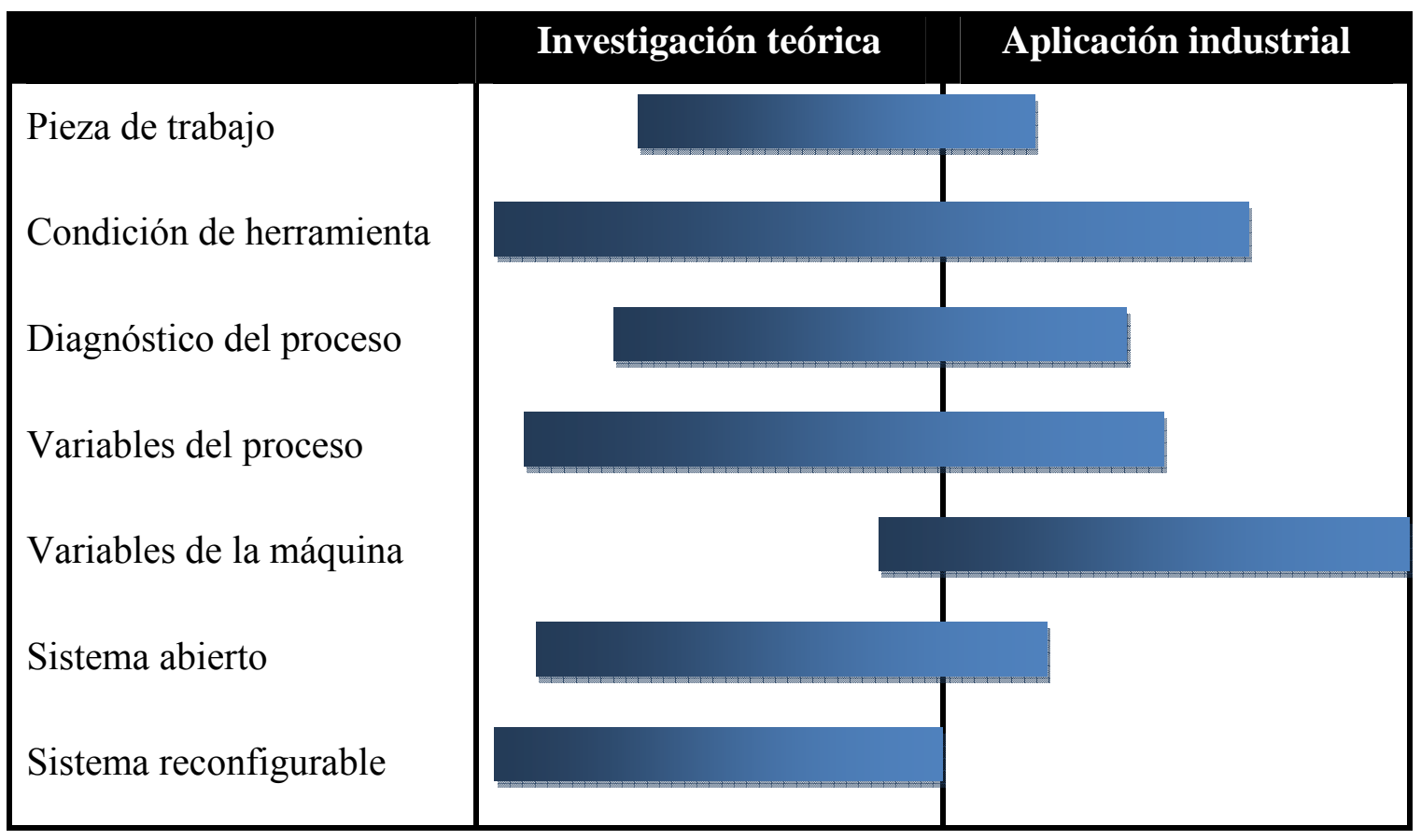

Tabla 2.2. Esquema del desarrollo de la monitorización de las distintas variables

La mayoría de los sistemas de monitorización se han desarrollado para procesos de complejidad limitada, como el taladrado o el roscado. Se han realizado estudios específicos para el proceso de fresado, midiendo fuerzas [23] o aceleraciones [24].

El control adaptativo es también una manera de llevar a cabo el proceso con un alto rendimiento y reducido nivel de errores.

A continuación se realizará un compendio de los conceptos más importantes que intervienen en la comprensión del fresado con los que se trabaja en la industria actual.

\subsubsection{Análisis de los parámetros del fresado}

En la investigación realizada se han estudiado las distintas modalidades de la operación, es decir, fresado en oposición y en concordancia, analizando las ventajas e 
inconvenientes que presenta cada una de ellas [6]. El fresado en oposición tiene la ventaja de que el filo no entra en contacto directo con la superficie de la pieza, evitando así el corte en zonas de material oxidado o endurecido. Como se sabe, la ventaja del fresado en concordancia es el menor consumo de energía, ya que la mayor parte del tiempo las fuerzas de corte van en la misma dirección del avance de la pieza. Se han realizado otras consideraciones [25] que aconsejan el fresado en oposición para operaciones con alta tasa de eliminación de material, ya que el corte en cada diente es gradual hasta alcanzar el espesor máximo de viruta.

Atendiendo a la posición del eje de la herramienta el proceso se clasifica en fresado cilíndrico y frontal [11]. El fresado cilíndrico es aquél en el que el eje es horizontal y se suele emplear para operaciones de planeado aplicado a toda la superficie de la pieza. El fresado frontal se emplea para una mayor variedad de operaciones, tales como contorneados o vaciados. Dado que los mecanizados que requiere el proceso de Microfresado suelen estar localizados en una parte pequeña de la pieza o en su contorno, el tipo de fresado que será de aplicación fundamentalmente será el frontal.

El número de dientes de las fresas puede ser muy diferente dependiendo del diámetro de la herramienta y de su robustez. En el presente trabajo, como se ha indicado, el número de dientes es 2 porque permite mejor evacuación de viruta con pequeños tamaños de herramienta. Por otro lado, el modelo con fresas de 2 dientes puede generalizarse fácilmente para otros casos, ya que con un modelo de 2 filos se caracterizan los efectos de los factores más importantes que actúan en el proceso.

En el trabajo de Shaw sobre el corte de metales [26] se establecen los principios del corte ortogonal y la relación de la geometría del corte con las fuerzas del mecanizado. En la figura 2.1 se muestra el esquema del corte ortogonal con los ángulos que lo caracterizan: ángulo de cizallamiento $\phi$, ángulo de desprendimiento de la herramienta $\gamma$ $\mathrm{y}$ ángulo de rozamiento $\tau$. La relación entre el espesor de viruta indeformada $\mathrm{y}$ deformada se denomina factor de recalcado:

$$
\xi=\frac{t_{2}}{t_{1}}(2.2)
$$




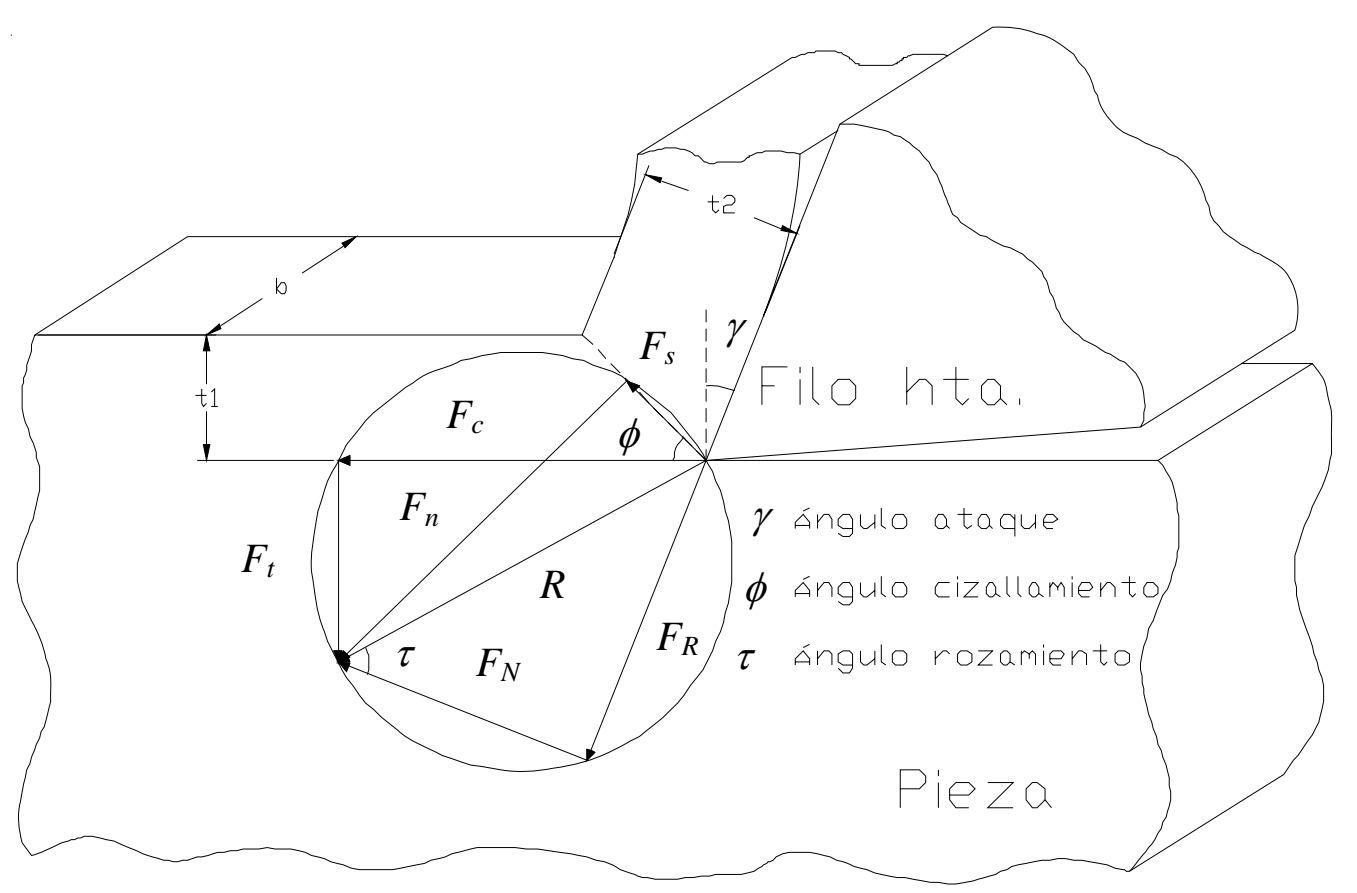

Figura 2.1. Esquema del corte ortogonal

Dado que el corte es un proceso de deformación plástica a cizalladura, las fuerzas dependerán de la tensión de fluencia a cortadura o tensión dinámica de cizallamiento $\tau_{s}$.

Merchant [27] desarrolló un modelo de corte ortogonal en el que se proporciona una relación entre el ángulo de cizallamiento y el ángulo de desprendimiento de la herramienta aplicando el principio de mínima energía al proceso. De esta manera se puede obtener el valor de las fuerzas de corte $F_{c}$ y $F_{t}$ en función de la tensión dinámica de cizallamiento y los ángulos de corte:

$$
\begin{aligned}
& F_{c}=f\left(\tau_{s}, \phi, \tau, \gamma\right) \\
& F_{t}=f\left(\tau_{s}, \phi, \tau, \gamma\right)
\end{aligned}
$$

Este modelo ha sido aplicado a la estimación de fuerzas en fresado, pero presenta numerosos problemas, ya que es difícil la determinación exacta del ángulo de cizallamiento, así como de la tensión dinámica de deslizamiento. 


\subsubsection{Modelos de fuerzas en el fresado}

El modelado y la simulación de los procesos de fresado son de gran utilidad en el diseño del proceso, la maquinaria y el análisis del fenómeno. También se emplean para determinar la eficiencia del proceso en términos de errores dimensionales, ya que permiten predecir la desviación de la herramienta respecto a su posición teórica debido a la acción deflectora de las fuerzas de corte.

Una posible clasificación de los modelos de fuerzas en el mecanizado es la que lleva a cabo el Grupo de Trabajo del CIRP "Modelling of Machining Operations", que se puede resumir en la siguiente relación de tipos de modelos:

1. Modelos semiempíricos

2. Modelos analíticos

2.1 Modelos mecanísticos

2.2 Modelos del plano de cizallamiento

3. Modelos numéricos

\section{- Modelos semiempíricos}

Los modelos semiempíricos se basan en la ecuación de Taylor [28], a la que se añaden diferentes parámetros como la profundidad de corte, avance o propiedades de los materiales para obtener la vida de herramienta en función de la velocidad de corte.

\section{- Modelos analíticos}

Los modelos analíticos consisten en la determinación de las fuerzas de corte mediante un análisis teórico del fenómeno de corte a partir de los principios de la mecánica del movimiento y de la tensión y deformación de los materiales. Este análisis se realiza a partir de las variables que definen el proceso, tales como el coeficiente de rozamiento, los parámetros de comportamiento elastoplástico del material de la herramienta y de la pieza de corte, las condiciones de generación y evacuación del calor, etc. 
Algunos modelos analíticos consisten en calcular la relación entre fuerza y espesor de viruta a partir de las expresiones (2.3) y (2.4) y de los parámetros incluidos en ellas [29,30]. Dado que hay algunos parámetros, como el ángulo de rozamiento o la tensión dinámica de cizallamiento que dependen de los materiales de herramienta y pieza utilizados, se debe proporcionar una tabla con los valores de esos parámetros para los distintos casos. Estos modelos se llaman modelos unificados de corte, ya que en ellos se consideran de manera global todas las variables que intervienen en el proceso y de los que dependen las fuerzas de corte.

Los modelos analíticos proporcionan distintos niveles de precisión en función de las simplificaciones que se consideren en el análisis [31] y van desde métodos de selección previa de herramienta [32] hasta los modelos de distribución de tensión y deformación en la zona de cizallamiento. Dentro de estos modelos destacan especialmente dos grupos, que se han utilizado de manera más extensa: los modelos mecanísticos y los modelos basados en el plano de cizallamiento.

\section{o Modelos mecanísticos}

Dentro de los modelos analíticos, los modelos mecanísticos pueden ser definidos como aquéllos basados en la mecánica del proceso de corte. Sin embargo, no son puramente analíticos y su capacidad de modelado depende en gran medida de datos de corte empíricos. Se puede decir que son modelos en los que se combinan las técnicas de modelado analítico y experimental. Este método tiene la gran ventaja de no requerir parámetros mecánicos del material como el ángulo de cizallamiento, la tensión dinámica de cizallamiento, el ángulo de rozamiento, etc., que habitualmente supone una gran complicación.

Dado que la vía analítica general para determinar las fuerzas de corte depende de parámetros difíciles de estimar (tensión dinámica de cizalladura, ángulo de rozamiento, etc.) y estos parámetros son muy sensibles a variaciones de condiciones como velocidad de corte o avance, en el caso concreto del modelo mecanístico se emplea un enfoque empírico, que consiste en relacionar la componente tangencial de la fuerza y el espesor de viruta indeformada mediante un coeficiente constante. Esta relación equivale a 
concentrar en una sola constante todos los términos que acompañan al espesor de viruta en las expresiones analíticas de las fuerzas de corte (2.3) y (2.4).

Desde este punto de vista, los modelos mecanísticos son de gran utilidad, ya que utilizan un número reducido de parámetros y requieren, por tanto, menos coste computacional para las aplicaciones de los modelos al control del proceso. Por otro lado, existe cierta falta de información referente a los parámetros analíticos de las fuerzas de corte, por lo que los modelos unificados son de utilidad relativa, mientras que los modelos mecanísticos tienen una clara ventaja debido a que los parámetros se determinan de manera empírica.

Un modelo de fuerzas mecanístico para el fresado consta, por tanto, de dos elementos fundamentales: un modelo geométrico y una relación entre la geometría y las fuerzas de corte, según el esquema mostrado en la figura 2.2. Las técnicas de modelado mecanístico se basan en las relaciones básicas que existen entre las fuerzas de corte y el espesor de viruta, la geometría de corte de la herramienta, las condiciones de corte, la geometría de la pieza y el tipo de operación. 


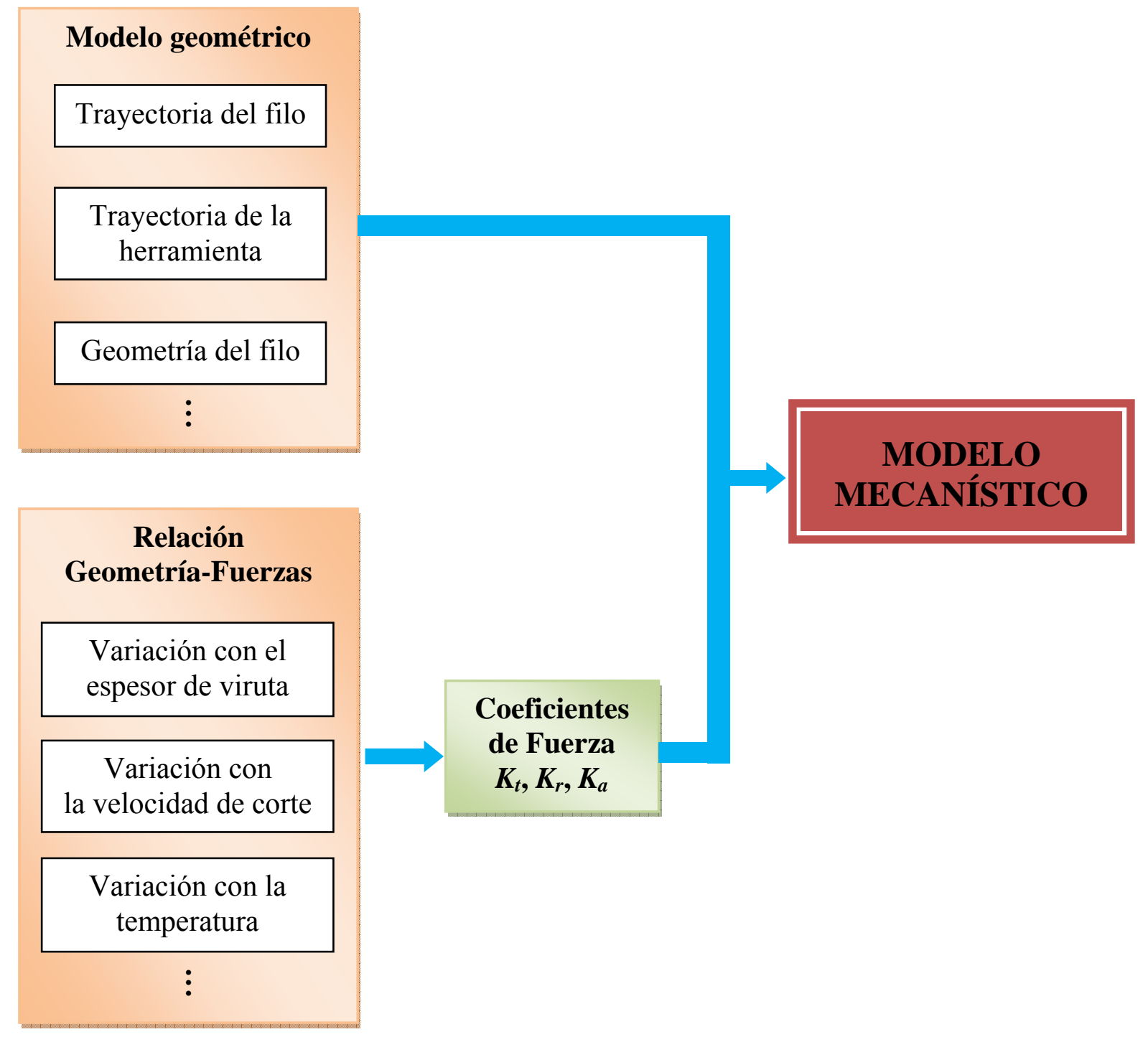

Figura 2.2. Esquema de un modelo mecanístico

Los primeros autores que modelaron el proceso de fresado utilizaron la siguiente ecuación empírica:

$$
F_{c}=k_{c} \cdot b \cdot h
$$

Donde $k_{c}$ es la fuerza específica de corte y es función de los parámetros de corte, tales como el espesor de viruta, el ángulo de desprendimiento, el ángulo de inclinación, etc. $b$ es la anchura de corte y $h$ es el espesor del corte. En esta línea se ha presentado [33] un modelo mecanístico para predecir las fuerzas de corte en el fresado en el que se incluye la consideración de la desviación excéntrica y la deflexión de herramienta para estimar 
la precisión de la pieza mecanizada. Posteriormente se mejoró este modelo para considerar la influencia de la deflexión de herramienta en el espesor de viruta [34].

Para la aplicación de un modelo mecanístico se deberán realizar varios ensayos con distintas velocidades de corte y avances por diente para la determinación de los coeficientes de fuerza antes de su utilización, ya que estos coeficientes varían con las condiciones aplicadas.

Tlusty y McNeil [35] proponen un modelo mecanístico en el que la fuerza de corte se calcula analíticamente en cada punto del recorrido del filo. Para ello se analiza la geometría de la trayectoria del filo y se calcula el espesor de viruta para cada ángulo de posición. El filo deberá dividirse en elementos diferenciales, cada uno de los cuales cortará un espesor de viruta diferente dependiendo de su ángulo de posición. En cada elemento diferencial de filo se multiplica el espesor de viruta por el coeficiente de fuerza. En este trabajo se propone una expresión del coeficiente basada en datos experimentales dependiente del avance por diente:

$$
K_{T}=C \cdot f^{p}
$$

Donde $C$ es la constante de fuerza, $f$ es el avance por vuelta y $p$ el exponente del avance por diente, que en este caso tiene valor negativo.

Los límites de integración de las fuerzas serán diferentes en función de la zona en la que se encuentre el filo. Se pueden distinguir tres zonas distintas A, B y C, según se indica en la figura 2.3). Los diferentes tramos corresponden a diferentes posiciones del filo con respecto a la zona mecanizada. Si el filo está entrando en la pieza y no ha llegado a tener contacto con ella en toda la profundidad de corte se halla en la zona A. Si el filo está totalmente introducido en la zona de corte y el contacto con la pieza se produce a lo largo de toda la profundidad de corte estará en la zona B. Cuando el filo esté saliendo de la pieza sólo tendrá contacto con la pieza en un tramo de la parte superior de la altura de corte y su situación corresponderá a la de la zona C. 
Para realizar la suma o integral de las fuerzas diferenciales se deberá proyectar cada una de ellas en la dirección $x$ o $y$, de tal manera que la suma corresponderá a la resultante de las fuerzas de corte en cada dirección. Esta resultante dependerá del ángulo de posición y tendrá oscilaciones importantes en función del número de dientes de la fresa y de la amplitud de la zona de corte. Si se multiplica la componente tangencial de las fuerzas diferenciales por el radio de la herramienta y se realiza la integral se obtendrá el par que ejerce la herramienta, a partir del cual podría obtenerse también la potencia.

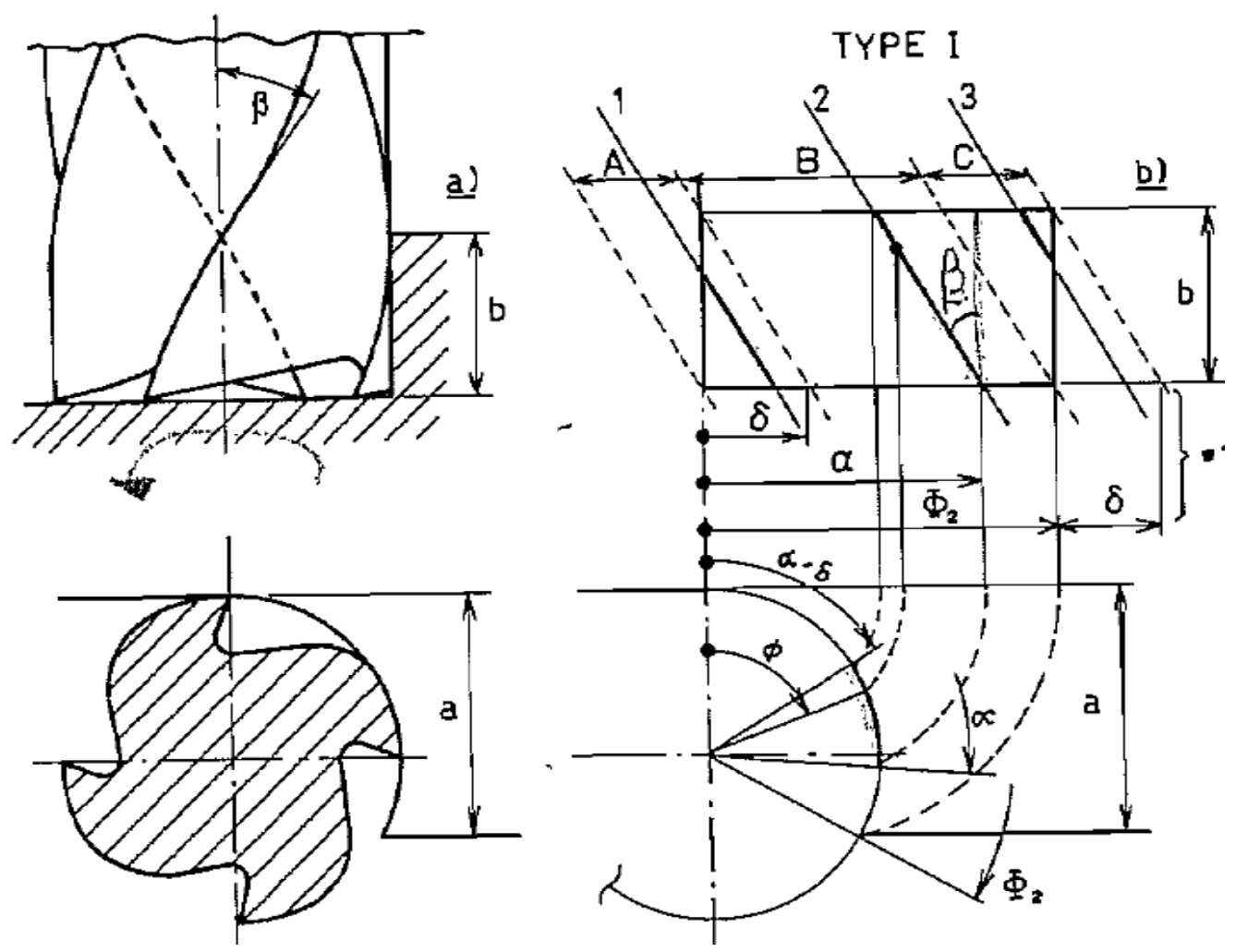

Figura 2.3. Distintas zonas del corte

La predicción de fuerzas mediante este modelo mecanístico es relativamente buena, según muestran los resultados.

Dado que en estos modelos la fuerza se calcula a partir del espesor de viruta y este espesor depende de la posición de herramienta, se deberá tener en cuenta cualquier variación de la posición de herramienta respecto al valor teórico. Un factor que modifica la posición de herramienta es la deflexión debida a la fuerza que ejerce en el corte. Por 
tanto, será necesario resolver un sistema de ecuaciones en el que intervenga el espesor de viruta y la deflexión de herramienta para obtener las fuerzas de corte. Esta influencia de la deflexión de herramienta hace que en régimen permanente se amortigüe el efecto de la desviación excéntrica de la herramienta, que provoca un desequilibrio entre los picos de fuerza debidos a los diferentes dientes de la fresa [36].

En régimen transitorio se observa que cuando la fresa está entrando en la pieza, la fuerza llega rápidamente a su valor máximo, pero este valor es inferior al que adquiere en régimen estacionario, por lo que los pulsos de fuerza son de menor duración. De manera análoga, ante cambios bruscos de la velocidad de avance, la reacción de las fuerzas es gradual y no adquiere su valor permanente hasta un ciclo o ciclo y medio después. En un ciclo de control adaptativo hay que tener en cuenta este retardo entre los cambios en la velocidad de avance y los cambios en la fuerza.

DeVor y otros [37] proponen una expresión para la estimación de la desviación de la herramienta debida a la deflexión:

$$
\delta_{R}=\frac{F_{R} \cdot a^{2} \cdot(3 L-a)}{6 \cdot E \cdot I}
$$

Donde $F_{R}$ es la fuerza de corte en dirección radial, $a$ la distancia del punto de aplicación de la fuerza al lugar de sujeción de herramienta, $L$ es la longitud de la herramienta, $E$ el módulo de elasticidad e $I$ el momento de inercia de la herramienta. Como se puede observar, la deflexión es proporcional a la fuerza resultante aplicada. El parámetro $a$ puede ser ignorado si la profundidad axial de corte es pequeña respecto a la longitud de herramienta, ya que la fuerza se puede considerar aplicada en la punta.

A diferencia de las expresiones propuestas por Tlusty y McNeil [35] existen otros modelos como el de Kline y otros [33], en el que la componente radial de la fuerza se considera proporcional a la componente tangencial mediante una constante llamada $K_{R}$.

En este trabajo $K_{T}$ y $K_{R}$ se determinan experimentalmente a partir de las fuerzas medias del proceso. No obstante, hay que tener en cuenta que se observa una gran variabilidad de las constantes con las condiciones de corte, sobre todo con la velocidad de avance. 
Esta variación se debe también al efecto tamaño, por el cual la presión específica de corte aumenta cuando disminuye el espesor de viruta. Por ello se realizó una base de datos de valores de $K_{T}$ y $K_{R}$ para distintas condiciones de corte. Estos valores se ajustaron a una curva polinómica de segundo orden en función del radio de la herramienta, la profundidad axial de corte y el avance por diente. El ajuste demostró ser bastante bueno.

Se puede establecer la relación entre la componente tangencial de la fuerza y el avance por diente con la siguiente expresión:

$$
d F_{t}=K_{T} \cdot f \cdot \sin \beta \cdot d z
$$

Donde $f$ es el avance por diente, $\beta$ el ángulo de posición y z la coordenada vertical. El coeficiente $K_{T}$ se relaciona con el avance por diente según la expresión (2.6). Dado que Sabberwal [16] demuestra que:

$$
\sin \beta^{p+1} \approx \sin \beta
$$

Englobando las constantes $C$ y $f^{p}$ de la expresión (2.6) en el coeficiente $K_{T}$ se confirma que la expresión (2.8) es correcta.

Se observa en los distintos estudios experimentales que el parámetro $K_{R}$ está muy influenciado por el desgaste de herramienta. En línea con los valores utilizados por Tlusty y McNeil [35], $K_{R}$ suele tener valores inferiores a 1, por lo cual, cuando se obtienen empíricamente estimaciones superiores a 1 se consideran casos especiales.

En el trabajo de Kline y otros [33] también se proporciona una correlación de la constante $K_{T}$ en función de los siguientes parámetros de corte: avance por diente, profundidad axial de corte y profundidad axial de corte.

Los modelos mecanísticos de predicción de fuerzas se utilizan frecuentemente para establecer la velocidad de avance en cada punto de la trayectoria de la herramienta para que las fuerzas máximas no excedan un cierto valor. Un sistema económicamente viable 
para realizar la regulación es llevarla a cabo en la zona de trayectoria curva, en la que la deflexión de herramienta provoca errores dimensionales de mayor entidad, manteniendo en el resto de tramos una velocidad predeterminada. De esa manera, se reduce la velocidad únicamente en los puntos críticos mientras que en el resto de la trayectoria la herramienta se mueve a velocidad económicamente rentable. Ésta es la primera aplicación existente de un modelo de fuerzas al diseño del proceso.

En la base de datos dada por Zlatin y otros [38] se demuestra que la fuerza de rotura aumenta con la profundidad radial de corte.

La desviación excéntrica de la herramienta tiene un efecto directo en el espesor medio de viruta, ya que aunque la cantidad de material eliminado es la misma, hay zonas en las que los dientes no cortan. Existe una relación empírica lineal entre la desviación excéntrica y el espesor medio de viruta. Esto obliga a revisar el procedimiento de estimación de las constantes de fuerza a partir de las fuerzas medias. En el trabajo de Kline y DeVor sobre la desviación excéntrica de la herramienta [39] las expresiones obtenidas para las constantes de fuerza en función del espesor medio de viruta son del siguiente tipo:

$$
K_{T}=K \cdot\left(\overline{t_{c}}\right)^{m}
$$

Las expresiones obtenidas en modelos que no consideran la desviación excéntrica de la herramienta son más complejas porque se necesita reflejar la influencia de dicho factor mediante otros parámetros de manera indirecta, como la velocidad de corte o la presión específica de corte. Por otro lado, Kline y DeVor demuestran que la influencia de la desviación excéntrica es mayor cuanto menos agresivo es el corte, es decir, cuanto menor sea el avance y la profundidad axial de corte.

Fu y otros [40] obtienen las componentes cartesianas de las fuerzas mediante la proyección de las componentes tangencial y normal, lo cual da lugar a una expresión matricial: 


$$
\left[\begin{array}{c}
F_{X}(i, \phi) \\
F_{Y}(i, \phi)
\end{array}\right]=\left[\begin{array}{cc}
\cos \theta_{i}(\phi) & -\sin \theta_{i}(\phi) \\
\sin \theta_{i}(\phi) & \cos \theta_{i}(\phi)
\end{array}\right] \cdot\left[\begin{array}{c}
F_{T}(i, \phi) \\
F_{R}(i, \phi)
\end{array}\right]
$$

La presión específica de corte cambia con el espesor de viruta, sin embargo, no es práctico tener en cuenta esta variación para la predicción de las fuerzas, ya que las expresiones de las fuerzas en función del espesor de viruta dejan de ser lineales y es mucho más difícil integrarlas. Por ello se suele emplear el espesor medio de viruta para la estimación de las constantes de fuerza, como hacen Koenigsberger y Sabberwal.

El modelo propuesto por Budak y otros [41] puede considerarse mecanístico, ya que los coeficientes de fuerzas se determinan sustituyendo las fuerzas promedio medidas en el proceso en las expresiones obtenidas en el modelo. Previamente es necesario haber determinado los coeficientes de fuerzas de aplastamiento $K_{t e}$ y $K_{r e}$, que se obtendrán de la extrapolación de las fuerzas al caso de avance nulo, en el que sólo existirá fuerza de aplastamiento y no de arranque de viruta.

Smith y Tlusty por su lado [42] establecen varios tipos de modelos mecanísticos según los factores que se incluyan en el análisis:

1. Modelos de fuerza promedio rígida con deflexión estática [43]

Estos modelos estiman la fuerza de corte a partir de la potencia media del proceso, con la cual obtienen la deflexión de herramienta y con ella un cálculo del error dimensional asociado.

2. Modelos de fuerza instantánea rígida $[35,37]$

Estos modelos hacen una estimación más exacta del valor de las fuerzas de corte mediante la suma de las fuerzas generadas por los elementos de filo involucrados en el corte en cada instante.

2.1. Modelos de fuerza instantánea rígida con deflexión estática $[44,45]$ La deflexión de herramienta se calcula sin intervención de la inercia. Este modelo es útil para determinar la posición de la superficie mecanizada, especialmente en las esquinas de los perfilados. 
2.2. Modelos de fuerza instantánea realimentados con deflexión estática $[34,46]$

Estos modelos consideran una progresión geométrica de espesores de viruta que generan los sucesivos dientes, de tal manera que se tiene en cuenta la deflexión de la herramienta en el paso de todos los dientes anteriores. Se considera además que la deflexión es constante a lo largo de toda la longitud de herramienta.

3. Modelos de fuerza regenerativa con deflexión dinámica $[47,48]$

Estos modelos toman en consideración la inercia de la herramienta, así como el amortiguamiento viscoso, para determinar las condiciones en las que surgirá el fenómeno del retemblado.

Kapoor y otros autores [49] proponen un modelo dinámico en el que se tiene en cuenta la deformación del conjunto pieza-sujeción además de la deflexión de herramienta, según se indica en la figura 2.4. Se consideran también cambios en la geometría de corte. Este modelo considera que la presión específica de corte y el coeficiente de rozamiento pieza-herramienta dependen del ángulo de desprendimiento, además del espesor de viruta y la velocidad de corte. 


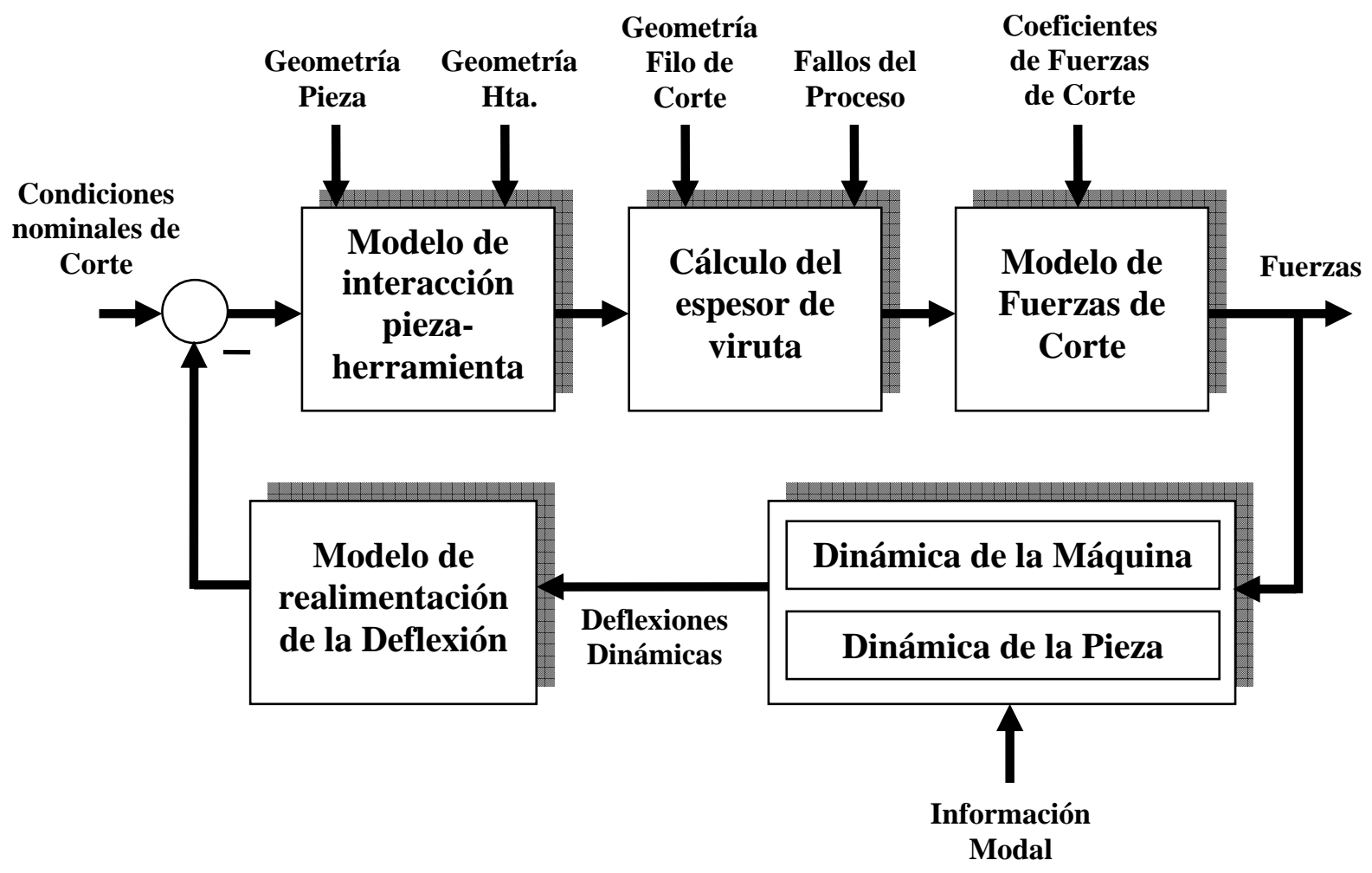

Figura 2.4. Esquema de un modelo de fuerzas dinámico

Según el trabajo [49], para tener en cuenta el fenómeno del aplastamiento debido al redondeo del filo se deben considerar deformaciones de naturaleza elástica hasta que el desgaste en flanco alcanza un determinado valor, momento tras el cual existirán deformaciones elásticas y plásticas. Esto implica que para herramientas nuevas, en las que el valor del desgaste es nulo o muy pequeño, no es necesario considerar una modificación de la posición de la superficie cuando el filo ejerza únicamente recalcado.

Los autores del trabajo que se viene citando [49] realizan un estudio de la dinámica del sistema basado en las condiciones de flexibilidad del conjunto herramienta-pieza, así como su amortiguamiento e inercia. Si la herramienta es flexible inevitablemente aparecerán vibraciones. En los resultados experimentales de fuerzas proporcionados se observan vibraciones de una frecuencia superior a la señal correspondiente a las fuerzas de corte oscilatorias, superpuestas a ella, lo cual indica que en el proceso aparecen vibraciones correspondientes a la frecuencia natural del sistema. Por tanto, si la frecuencia del proceso de corte se superpone con la frecuencia natural del sistema aparecerá el fenómeno de la resonancia, como se analizará en el capítulo 8. 
Este estudio [49] también revela que los coeficientes de fuerzas varían con el espesor de viruta de manera más continua en el fresado en oposición que en el fresado en concordancia, quizá debido al comienzo brusco de corte que presenta esta última modalidad.

Para tener en cuenta la deflexión de herramienta se hace una realimentación de los resultados de fuerza para calcular la desviación de la herramienta y con este valor se recalcula la fuerza de nuevo, como se indica en el esquema de la Figura 2.5. Lo mismo ocurre con la consideración de la dinámica del sistema, por lo cual, los modelos pueden representarse mediante un sistema regulado en el que el bucle de realimentación está constituido por la deflexión de herramienta y la dinámica del sistema.

Según esta investigación [49], los modelos dinámicos que consideran las vibraciones del sistema se llamar de fresado vibratorio, ya que la forma de abordar el fenómeno es muy diferente de la estática. Se ha descubierto que en la construcción del modelo dinámico el fenómeno de recalcado o aplastamiento es de gran relevancia, ya que actúa como un importante factor de amortiguamiento.

Por otro lado, se observa que las marcas de las vibraciones en la superficie mecanizada se evitan estableciendo una relación entera entre la velocidad de giro del husillo y la frecuencia natural del sistema herramienta-pieza.

El inconveniente de los modelos mecanísticos es que es preciso recalcular los coeficientes de fuerzas cada vez que cambia la geometría del corte o los materiales.

o Modelos del plano de cizallamiento

Los modelos del plano de cizallamiento tienen su origen en el análisis del corte ortogonal que se ha expuesto en el apartado anterior mediante las expresiones (2.3) y (2.4). Se ha aplicado este análisis al caso del fresado y se han deducido las fuerzas de corte por medio de la geometría del proceso. Han surgido muchas objeciones a estos modelos debido a las dificultades teóricas que presenta la consideración del plano de cizallamiento. Sin embargo, se han corregido de manera satisfactoria estas dificultades mediante trabajos como $[50,51]$. 
El modelo propuesto por Armarego y otros [50] consiste en un sistema de predicción de fuerzas para el fresado frontal con insertos dividiendo los dientes en elementos y sumando las fuerzas en cada uno de ellos como se hace para el fresado con herramientas enterizas. Este modelo considera dos términos en el cálculo de la fuerza del proceso: la fuerza de corte y la fuerza del filo, que se ajustan a la siguiente expresión:

$$
d F_{i j}=\left(K_{c k} \cdot t_{n i j}+K_{e k}\right) \cdot d b_{i j}
$$

El primer término se debe a la cizalladura correspondiente al arranque de viruta mientras que el segundo se debe a la fuerza de recalcado y rozamiento que ejerce el filo sobre el material.

Por lo que respecta a los errores en el fresado frontal existen dos tipos de errores de posición de los filos: la excentricidad de la herramienta, debida a la desviación del eje geométrico de la herramienta respecto al eje del husillo y el error debido a la colocación incorrecta de las plaquitas en la fresa. El primer error presenta una variación senoidal mientras que el segundo es aleatorio. Experimentalmente se demuestra que el segundo tipo de error es el predominante. En este trabajo [50] la variación aleatoria de diámetro se toma directamente de medidas experimentales sobre la fresa.

Éste es un modelo de mecánica unificada de corte, dado que obtiene las constantes de fuerza mediante una base de datos de parámetros generales de corte aplicados a la geometría del fresado.

La simulación del proceso según este modelo presenta tendencias plausibles de las fuerzas de corte respecto a los parámetros de corte, como la disminución de la fuerza con el ángulo de desprendimiento. Los resultados de la simulación muestran que las fuerzas promedio para los modelos con y sin desviación excéntrica de la herramienta son comparables.

Una combinación de condiciones interesante del fresado es aquélla en la que las fuerzas son constantes, como explican Armarego y Deshpande [36], debido a que la longitud de 
filo implicada en el corte es constante y correspondiente a toda la profundidad axial de corte:

$$
\begin{array}{r}
a_{p}=\frac{m \cdot 2 \pi R}{Z \cdot \tan \beta} \\
m=1,2,3, \ldots
\end{array}
$$

Estas condiciones hacen posible la estimación de las constantes de fuerza de una manera sencilla para herramientas con suficiente longitud de filo como para cumplir la relación anterior.

En este último trabajo [36] se señala que la desviación excéntrica de la herramienta es debida principalmente a inexactitudes en el sistema de sujeción de herramienta, aunque existan también errores en la fabricación de las fresas.

Este modelo [36] considera que la deflexión provoca una desviación del centro de la herramienta proporcional a la fuerza ejercida por ésta y en la misma dirección, que se suma a la desviación excéntrica de la herramienta. Esto provoca una distorsión en el espesor de viruta que afecta a las fuerzas de corte. Se asume la simplificación de que la deflexión no afecta a la dirección de los radios de posición de los dos filos que intervienen en la determinación del espesor de viruta.

Un inconveniente que tienen los modelos analíticos o de mecánica unificada de corte es que no predicen bien las fuerzas en condiciones dinámicas. Por otro lado, como se señaló anteriormente, se necesita una gran base de datos de parámetros para obtener los coeficientes de las fuerzas, datos que no están siempre disponibles, dado que varían mucho con las condiciones de corte, tales como la velocidad de corte, el avance por diente, la temperatura, etc.

\section{- Modelos numéricos}

En estos modelos se emplean métodos numéricos, tales como el de elementos finitos, para calcular las variables en toda la zona del proceso. Estos modelos tienen el 
inconveniente de que muchas veces el patrón de comportamiento que emplean para el material está incompleto. Por otro lado, suelen requerir un tiempo excesivo de computación para extraer los resultados. Los modelos numéricos proporcionan un buen complemento a los modelos analíticos porque proporcionan análisis interesantes de ciertas tendencias en las fuerzas [52-55].

\subsubsection{Investigación específica en Microfresado}

Los aspectos más relevantes de la investigación en microfresado se pueden resumir en los siguientes puntos:

\section{A. Aplicaciones y ventajas del proceso \\ B. Características específicas del microfresado \\ C. Estudio geométrico \\ D. Influencia de la desviación excéntrica de herramienta \\ E. Desgaste y vida de herramienta \\ F. Efecto tamaño \\ G. Modelos de fuerzas desarrollados}

A continuación se desarrollará cada uno de estos puntos.

\section{A. Aplicaciones y ventajas del proceso}

La principal aplicación del proceso de microfresado ha sido el mecanizado de moldes, máscaras para su uso en la técnica de litografía y componentes estructurales de microsistemas, debido a su gran flexibilidad. El microfresado puede usarse también como un proceso rápido para fabricar estructuras con geometrías de gran relación profundidad-anchura [56].

Los procesos de micromecanizado se aplican también al desarrollo de prototipos y en la fabricación de elementos en los que se deben integrar las escalas mili y micro.

Las técnicas actuales de fabricación de microsistemas son las aplicadas, al menos en muchos casos, a la fabricación de circuitos integrados, y por ello son útiles, bien a la 
fabricación de grandes series, bien a la fabricación de lo que se viene denominando "componentes en dos dimensiones". Sin embargo la aplicación de tecnologías convencionales para diversos casos, tales como cuando se precisan sólo pequeñas o medianas series, en la fabricación de componentes en tres dimensiones, las tecnologías convencionales están resultando ser útiles y en particular el microfresado por ser un proceso, a la vez económico y flexible [57].

Otra gran ventaja que presenta el microfresado es debida a que hay materiales que se consideran no mecanizables a escala convencional, que lo son a pequeñas escalas, como los cerámicos.

La elección del microfresado como objeto de la investigación, se puede fundamentar en los siguientes hechos:

a.- La utilización del proceso convencional de remoción de viruta en el desarrollo de modelos de corte a escala micrométrica muestra un punto de partida al análisis muy prometedor.

b.- El microfresado ofrece una gran variedad de posibilidades de mecanizado [58][59].

c.- Desde el punto de vista económico, es una tecnología flexible y económica si el tamaño del lote no es muy elevado en la fabricación de micropiezas [60].

d.- Es una técnica muy interesante para operaciones de microacabado, el cual es un proceso altamente demandado industrialmente.

La investigación en microfresado se inicia en los años 60 y 70 con el mecanizado de ultraprecisión [2] que utilizaba exclusivamente el diamante como herramienta para obtener los acabados superficiales que eran necesarios. Sin embargo, la alta afinidad del diamante con el hierro hace imposible el uso de esta herramienta para el mecanizado de aceros, por lo que en el mecanizado de estos materiales, el diamante se sustituye por el carburo de wolframio.

La necesidad de utilizar el acero como material en la fabricación de micropiezas, se justifica especialmente en la fabricación de micromoldes y otros componentes de gran importancia en la fabricación de microsistemas [61]. 
Aunque el proceso de microfresado de metales ha sido objeto de múltiples investigaciones desde hace años [62], los resultados obtenidos apenas muestran que las estructuras obtenidas son con frecuencia aceptables sin mostrar mayor correlación entre las condiciones de corte y los acabados superficiales.

\section{B. Características específicas del microfresado}

Inicialmente la investigación en microfresado trató de extrapolar los modelos de corte del fresado al microfresado. Sin embargo, aunque se han obtenido ciertas aproximaciones, las diferencias son importantes, y pueden llegar en ciertos casos a superar el $50 \%$. Las razones son, que en el microfresado el tamaño del grano de la pieza condiciona de manera mucho más importante que en el fresado convencional, tanto las condiciones como el resultado del mecanizado [63]. Análogamente, el tamaño del grano del material de la herramienta puede condicionar ciertos aspectos de su geometría. Por lo demás, las condiciones de corte deben establecerse en campos de variabilidad mucho más estrechos que el fresado convencional.

Otro factor que introduce grandes diferencias entre el fresado convencional y el microfresado es la distorsión que provoca el calor desprendido en el proceso. Debido a esto, el control del calor desprendido es importante en el micromecanizado en general.

Por otro lado, la fuerza radial aumenta drásticamente cuando se pasa del fresado convencional al microfresado en relación al el espesor de viruta. Ésa es la causa de las frecuentes roturas de herramienta en este proceso.

El micromecanizado viola uno de los principios de la Ingeniería de Precisión, que establece tolerancias máximas de 1 unidad por 10000 en las dimensiones de la pieza [56]. En micromecanizado, en cambio, son normales unas tolerancias de algunos $\mu \mathrm{m}$ en dimensiones nominales de decenas de $\mu \mathrm{m}$. Sin embargo, en muchos casos no se necesita una precisión mayor en las piezas micromecanizadas.

En el micromecanizado las fuerzas son mucho mayores que lo que cabría esperar proporcionalmente del mecanizado convencional reduciendo el espesor de viruta. Esto es debido al redondeo del filo, que provoca que el ángulo de desprendimiento sea 
mucho mayor.

El microfresado se lleva a cabo con herramientas de diámetro inferior al milímetro hasta de $0.05 \mathrm{~mm}$ [64]. En este proceso las condiciones son mucho más agresivas que en el fresado convencional debido a que el avance por diente es mucho mayor respecto al radio de la herramienta. Esto hace, por ejemplo, que la vida de herramienta en el microfresado de aceros inoxidables corresponda a una longitud de pasada de unos 2500 mm [65]. Por tanto, la elección de condiciones de corte es mucho más crítica que en el fresado convencional y si no se realiza correctamente la herramienta puede durar un periodo de escasos segundos.

Durante el mecanizado pueden darse casos de filos dañados o incluso rotos que no se adviertan, debido a que la eliminación de material es a veces inapreciable a simple vista. Hay datos experimentales que indican que la fuerza radial en el microfresado es un $30 \%$ de la fuerza tangencial cuando se mecaniza aluminio [66,67]. Esta relación variará para otros metales [52].

\section{Estudio geométrico}

En el fresado convencional se desprecia el movimiento de avance frente al de rotación, debido a la baja relación $f_{z} / R$, por lo que se considera una trayectoria circular del filo. Sin embargo, en algunos casos del microfresado esta relación es mayor y por tanto no se puede despreciar, debido a que esta relación determina la tasa de producción del mecanizado. Por ello, en estos casos conviene considerar una trayectoria trocoidal en los filos, para aproximarse mejor al fenómeno real.

El modelo CEMO (Conventional End-Milling Operation) mencionado en la sección anterior es un caso particular del modelo MEMO (Micro End-Milling Operation) propuesto por Bao y Tansel [65], coincidiendo ambos cuando el avance por diente es mucho menor que el radio de la herramienta. Se puede observar además que ambos modelos conducen a resultados prácticamente iguales cuando $f_{z} / R<0.1$.

Este modelo puede servir para controlar el estado de la herramienta en tiempo real mediante el cálculo de los coeficientes de fuerza de corte en cada filo a partir de las 
medidas experimentales de las fuerzas. El incremento de estos coeficientes indicaría desgaste en la herramienta o incluso rotura. Se trata, por tanto, de un modelo mecanístico, que proporciona buenos resultados en su aplicación al control del proceso. También se pueden usar estos modelos mecanísticos en microfresado para establecer las condiciones de corte óptimas mediante el uso de la predicción de fuerzas que proporciona este modelo.

\section{Influencia de la desviación excéntrica de herramienta}

La desviación excéntrica de la herramienta en el microfresado tiene mayor importancia que en el fresado convencional, debido a que su valor es más significativo con respecto al radio de la herramienta por ser éste más pequeño [68]. Este fenómeno puede hacer que en una herramienta de dos filos uno de los filos no corte. Ello provocaría un incremento notable en la oscilación de las fuerzas y en las posibilidades de rotura.

El mejor indicador de la desviación excéntrica de la herramienta es la diferencia de magnitud que se observa entre los picos de fuerza. Si no hubiese desviación excéntrica los picos tendrían igual valor. Se observa que las variaciones de la fuerza son máximas cuando la línea de desviación de la herramienta está alineada con alguno de los filos y son mínimas cuando el ángulo entre estas dos líneas es máximo.

En algunos casos la deflexión de herramienta contrarresta el efecto de la desviación excéntrica de la herramienta, haciendo que el desequilibrio entre las fuerzas sea muy pequeño.

Un modelo analítico de fuerzas de corte puede usarse para estimar la desviación excéntrica a partir de los datos experimentales de la fuerza de corte, ya que este factor viene determinado por dos parámetros: la magnitud de la desviación $\rho \mathrm{y}$ su ángulo $\lambda$. Esta desviación provoca que uno de los filos tenga más distancia al eje que la nominal y por tanto, el borde de la superficie mecanizada esté más alejado del centro de la herramienta de lo que cabría esperar.

\section{E. Desgaste y vida de herramienta}


El principal motivo de sustitución de herramienta en microfresado es la rotura debido a las fuerzas de corte. Por tanto, cuando se habla de vida de herramienta se hace referencia a la fuerza máxima que ésta puede soportar, que se calcula teórica o experimentalmente. El desgaste tiene influencia en la vida de herramienta, pero sobre todo como factor que aumenta las fuerzas de corte y determina el tiempo que la herramienta va a poder seguir utilizándose sin romperse. El fenómeno del desgaste acelerado debido a las altas temperaturas no es significativo en el microfresado, aunque sí lo es en el fresado convencional.

El desgaste se modela añadiendo un factor de corrección a la fuerza unitaria del modelo $F_{u}$, que dependerá de la longitud de mecanizado que ha llevado a cabo la herramienta, según proponen Bao y Tansel [69]. Esto se confirma experimentalmente observando la forma de las gráficas de la fuerza de corte frente al tiempo. En el citado trabajo los parámetros $C_{1}$ y $C_{2}$ del factor de corrección de la fuerza fueron obtenidos por medio de algoritmos genéticos y se observó un buen ajuste con los datos experimentales. Estos parámetros fueron muy similares de un ensayo a otro, lo cual confirma que el modelo de desgaste propuesto es correcto.

En base al modelo de desgaste se puede hacer una estimación de la vida de herramienta, que coincide de manera satisfactoria con las mediciones experimentales. Este modelo permite una monitorización en tiempo real de la vida de herramienta, ya que en cada momento del mecanizado se puede realizar una estimación de los parámetros del factor de corrección mediante algoritmos genéticos.

\section{F. Efecto tamaño}

El arranque de viruta sólo tiene lugar cuando su espesor está por encima de un cierto valor y por tanto no se produce siempre que la herramienta y la pieza están en contacto [70]. Por tanto, existirá un ángulo en el que la herramienta comienza a cortar. Para valores inferiores del ángulo no se produce arranque de material. Este factor resulta perjudicial para el proceso, ya que una posible causa de la rotura de herramienta en el microfresado es el continuo cambio de recalcado a cizalladura en la acción de la herramienta. 


\section{G. Modelos de fuerzas desarrollados}

En un modelo analítico de fuerzas frecuentemente se considera la componente axial independiente de las otras dos, ya que se puede integrar sin proyectarla en las otras direcciones.

El modelo propuesto por Pérez y otros [71] se basa en la presión específica de corte, que se expresa como función potencial del espesor de viruta, de manera análoga a la función que se usa para expresar en los modelos mecanísticos del fresado el coeficiente de fuerzas respecto al avance por diente indicada en la expresión (2.6). Sin embargo, en este modelo se transforma esta relación en una de tipo lineal, despreciando en el desarrollo de Taylor los términos segundo y superiores, debido al pequeño valor del espesor de viruta. La expresión que se deduce es la siguiente:

$$
k_{t}=b \cdot h+c
$$

En este modelo a la fuerza tangencial calculada mediante esta presión de corte se le aplica un factor de corrección que expresa el error de posición de cada filo debido a imprecisiones de la fabricación de la herramienta. Los parámetros del modelo son ajustados mediante las mediciones de fuerzas en la primera vuelta de la herramienta. Con este modelo se puede llevar a cabo un control adaptativo del proceso.

El análisis expuesto por Newby y otros [72] proporciona expresiones empíricas para los coeficientes de fuerzas en función del espesor medio de viruta. Estas expresiones son distintas para el fresado convencional y para el microfresado.

Dado que las herramientas son delgadas y con una estrecha conexión con la zona de corte, la deflexión es un factor crucial que afecta a las fuerzas de corte [64].

Bissacco y otros [64] señalan que los coeficientes de fuerza resultan estimados por debajo de su valor real si se obtienen a partir de ensayos de corte ortogonal considerando ángulo de desprendimiento nominal, por lo que la utilización de modelos mecanísticos presenta una gran ventaja a estas escalas. 
En este trabajo se propone un modo de corregir el método de la estimación de los coeficientes de fuerza introduciendo el factor espesor de viruta - avance por diente en la determinación del ángulo efectivo de desprendimiento.

\subsubsection{Análisis dinámico}

En todo proceso de mecanizado existen vibraciones procedentes de la respuesta dinámica del sistema al elemento giratorio, que puede corresponder a la pieza de trabajo o a la herramienta de corte. Estas vibraciones aparecerán en el modelo de fuerzas del proceso siempre que se considere un comportamiento elástico de la herramienta [73].

Debido a esta acción conjunta de las variables de fuerza y posición aparece el fenómeno de las vibraciones autoexcitadas o retemblado [74]. En esta situación las fuerzas crecen de manera incontrolada hasta el límite impuesto por el amortiguamiento del sistema. En la figura 2.5 se puede ver la evolución de las fuerzas de corte en una situación de retemblado, en la que tiene lugar un aumento incontrolado de las valores, muy por encima de la magnitud habitual en el proceso. 

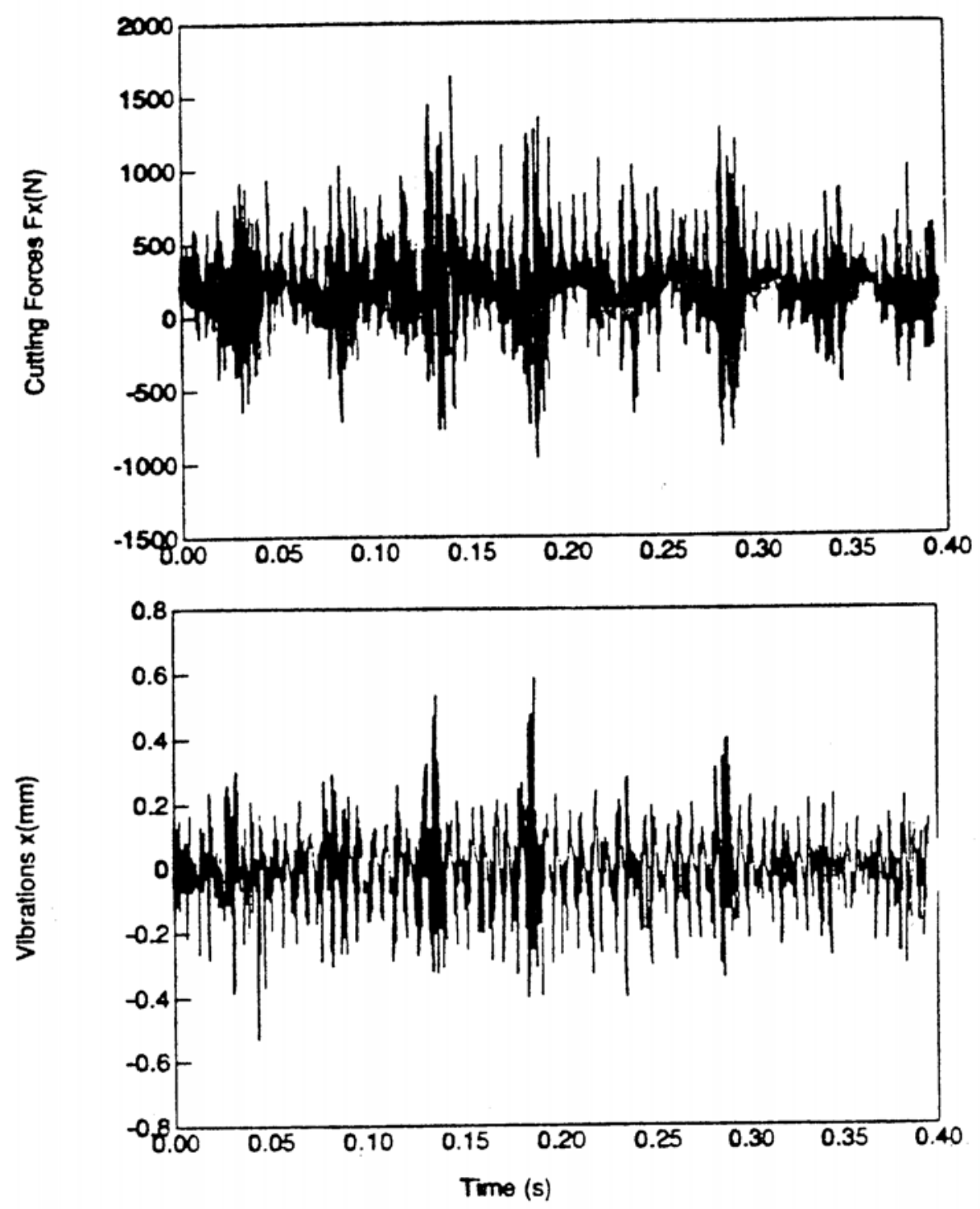

Figura 2.5. Evolución de fuerzas y vibraciones en situación de retemblado

Tlusty [75] y Tobias [76] establecieron la teoría fundamental de la estabilidad frente a retemblado, que proporciona una noción fundamental del corte en condiciones dinámicas y de los lóbulos de estabilidad frente al retemblado. Posteriormente Tlusty presentó una revisión general de la investigación en el corte dinámico [77]. Este estudio se centró en el modelado y la medición de los coeficientes de corte dinámico y su influencia en la estabilidad frente a retemblado en los procesos de corte con un solo punto de contacto entre herramienta y pieza. Posteriormente Rivin presentó un trabajo de recopilación de investigación sobre la dinámica de la interfaz husillo-herramienta [78]. Para el estudio del fenómeno se han empleado modelos basados en el método de los elementos finitos, así como modelos mecanísticos [25]. 
Desde la aparición del trabajo de Tlusty ha habido avances muy significativos en el modelado de las vibraciones en los procesos de mecanizado. Con los avances en computación, sensores y tecnología de máquinas herramienta de alta velocidad han aparecido nuevos métodos para la predicción y eliminación de las vibraciones de retemblado a nivel industrial. Altintas y Weck [79] realizan una revisión de los modelos matemáticos más importantes de corte en condiciones dinámicas, predicción de la estabilidad frente al retemblado para distintas operaciones y técnicas de prevención del retemblado dentro y fuera de proceso utilizadas con éxito en laboratorios y en la industria.

En el caso del torneado la pieza de trabajo es una barra giratoria de la que se elimina material por medio de una herramienta estática. Los desequilibrios de masa de la barra y la falta de concentricidad de la sujeción provocan la existencia de vibraciones que se reflejarán en las fuerzas de corte.

Partiendo del hecho de que el fresado pertenece al grupo de operaciones de contacto múltiple entre herramienta y pieza donde el proceso es intermitente y periódico según los intervalos de paso de filo de herramienta, Tlusty y sus colaboradores han contribuido a crear un conocimiento en profundidad del retemblado en el proceso de fresado y su eliminación. Otros investigadores han proporcionado métodos complementarios y una visión adecuada de la dinámica del fresado que conjuntamente con los anteriores han conducido a una eficaz utilización de este conocimiento en la industria con mejoras significativas de productividad.

En el caso del fresado la herramienta es un elemento giratorio también que puede tener desequilibrio estático de masas. Por otro lado pueden existir errores de ajuste en el sistema de sujeción de la herramienta. Estos factores causan una vibración en la posición de la herramienta cuando ésta está girando. Dado que las fuerzas de corte son proporcionales al espesor de viruta y éste a su vez depende de la posición de herramienta la vibración se traducirá en una variación oscilatoria de la fuerza de corte.

Por otro lado, la pieza es un sistema dinámico que presenta una determinada respuesta ante los esfuerzos que dependerá de su elasticidad, su amortiguamiento y su masa. En 
este sistema actuarán las fuerzas de corte que serán de naturaleza oscilatoria debido a la geometría del proceso. A dichas fuerzas se superpondrán las vibraciones provocadas por el movimiento oscilatorio de la herramienta. En suma actuará una fuerza variable sobre el sistema dinámico y éste generará una respuesta que puede ser estudiada mediante una función de transferencia asociada a la pieza que proporciona una salida dependiente de la entrada. Esta respuesta consistirá en un movimiento vibratorio.

Este movimiento de la pieza afectará al espesor de viruta, que a su vez influirá en las fuerzas de corte, por lo que el sistema vibratorio será una conjunción de factores cuyas variables están acopladas. Un sistema dinámico como la herramienta o la pieza puede representarse de manera simplificada mediante una masa, un resorte y un amortiguador viscoso [9], según se muestra en la figura 2.6.

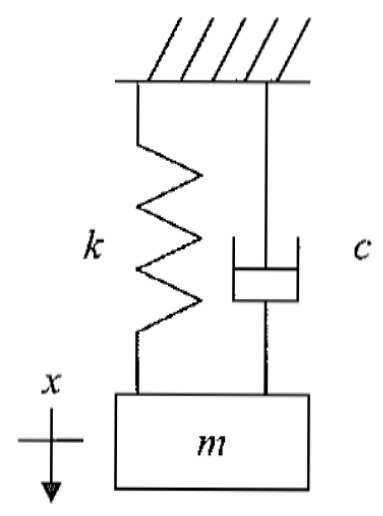

Figura 2.6. Esquema de un sistema dinámico

Este sistema presentará una frecuencia de vibración natural que responde a la fórmula:

$$
f=\frac{1}{2 \pi} \cdot \sqrt{\frac{k}{m}-\frac{c^{2}}{m^{2}}}
$$

Esta frecuencia deberá ser tenida en cuenta a la hora de establecer la velocidad de giro de la máquina, puesto que si la frecuencia de oscilación de las fuerzas coincide con ella se producirá el fenómeno de la resonancia. 
El mismo esquema puede utilizarse también para representar el conjunto herramientapieza, en el que cada elemento tendrá sus propios parámetros, como se muestra en la figura 2.7 .

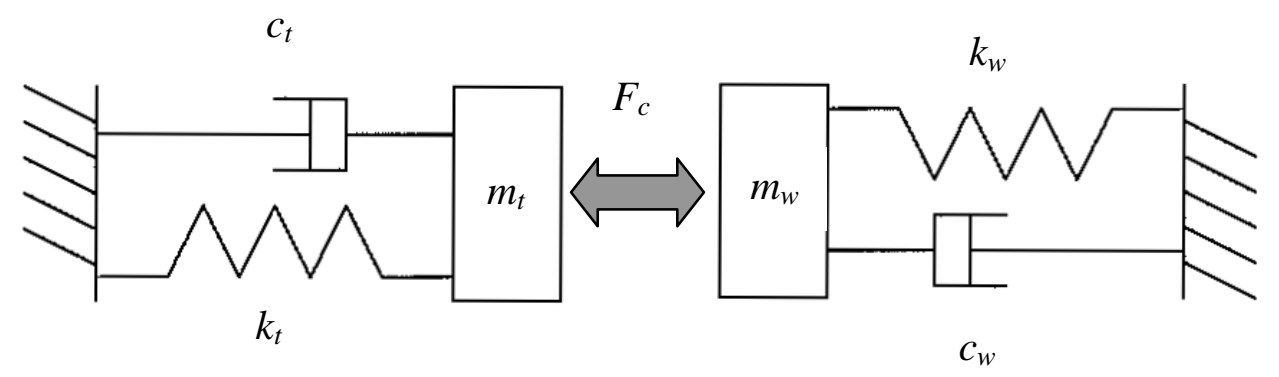

Figura 2.7. Esquema del conjunto herramienta-pieza

En la figura 2.7 aparecen los dos sistemas dinámicos interactuando a través de las fuerzas de corte, que dependen de la posición de ambos elementos e influyen a su vez en su movimiento. Este esquema es similar al utilizado por Newby y otros [73], que establecen un modelo de cálculo analítico de fuerzas en el que interviene la posición oscilatoria de la herramienta y la pieza. En este trabajo se señala que los modelos de fresado en los que se considera el comportamiento dinámico del sistema se denominan de fresado vibratorio y difieren en gran medida de aquéllos que hacen un análisis estático.

Altintas [79] proporciona un modelo dinámico de la herramienta bidimensional, ilustrado en la figura 2.8. Se asume que el avance de la herramienta se da en la dirección $x$. Para simplificarlo se desprecia la componente axial de la fuerza y se consideran, por tanto, dos fuerzas que actúan en la herramienta, correspondientes a los vectores giratorios de la fuerza tangencial y la fuerza normal. 


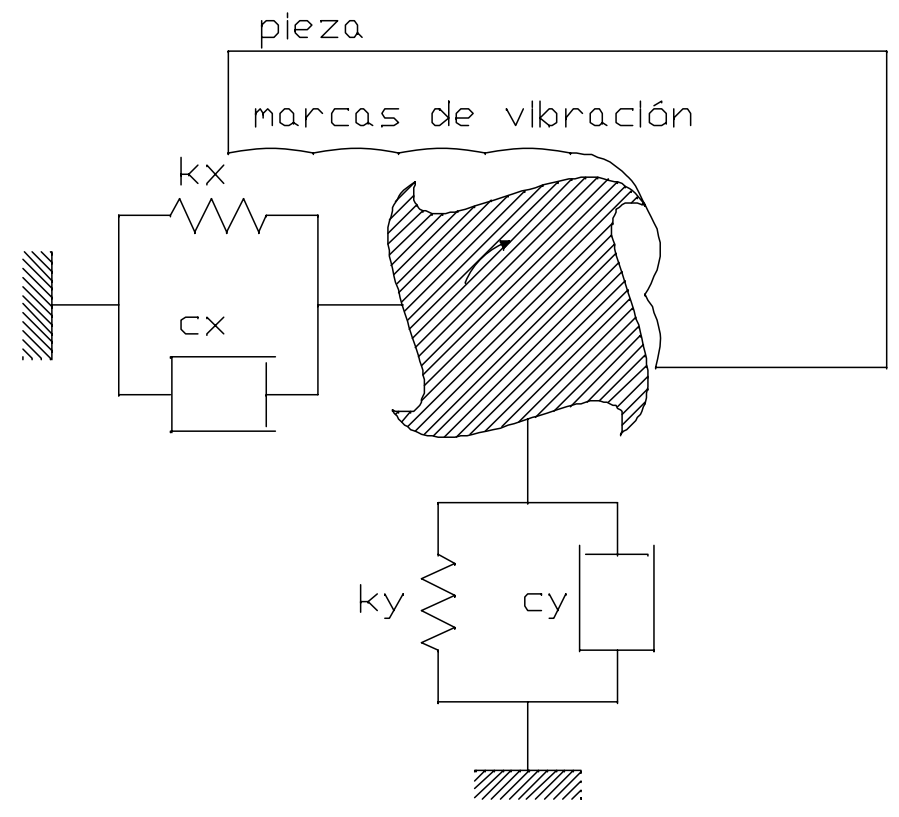

Figura 2.8. Modelo bidimensional de vibraciones en el fresado

En este modelo se consideran como parámetros dinámicos los correspondientes a la rigidez de la herramienta y la amortiguación viscosa en las direcciones $x$ e $y$. Según Altinas y Budak [80] la expresión que define el espesor de viruta es función del ángulo de posición de la herramienta, del avance por diente y de los desplazamientos $\Delta x$ y $\Delta y$ provocados por la vibración de la herramienta en los ejes $x$ e $y$ :

$$
h[\phi(t)]=f\left[f_{z}, \phi(t), \Delta x(t), \Delta y(t)\right]
$$

Las componentes de la vibración $\Delta x$ y $\Delta y$ varían según la frecuencia de vibración $\omega_{c}$, por lo que la fuerza resultante de corte se puede expresar de la siguiente manera:

$$
F(t)=F \cdot e^{i \omega_{c} t}
$$

Las vibraciones en el instante $t$ y en el mismo instante del diente anterior $t-T$ se pueden someter a un análisis similar al que realiza Altintas [25], del que surgirían las siguientes relaciones:

$$
\Delta x=f\left(F_{x}, \phi_{x x}\right)
$$




$$
\Delta y=f\left(F_{y}, \phi_{y y}\right)
$$

Donde $\phi_{x x}$ y $\phi_{y y}$ son las funciones de respuesta en frecuencia de la estructura vibratoria en las direcciones $x$ e $y$. Dado que las fuerzas de corte $F_{x}$ y $F_{y}$ dependen a su vez de la posición debida a las vibraciones, el sistema presenta una dinámica acoplada, como se señaló anteriormente. No obstante, Tlusty [81] simplificó la caracterización utilizando la dirección de la fuerza resultante como dirección de vibración.

El fenómeno del recalcado del material durante el corte constituye un fuerte factor de amortiguamiento, por lo que si el modelo de fuerzas lo ignora la amplitud de las vibraciones aumenta de modo drástico.

Si no se evitan las vibraciones surgirá en la pieza un acabado superficial inaceptable y carga excesiva en la herramienta y en el husillo, que pueden provocar la rotura de la herramienta o de los cojinetes. Es importante distinguir entre el retemblado provocado por vibraciones autoexcitadas y las vibraciones externas o forzadas durante el mecanizado e identificar claramente su causa [82]. Las vibraciones externas se pueden identificar observando el espectro de vibraciones. Estas vibraciones aparecen a la frecuencia de giro del husillo o del paso de dientes. Sin embargo, las vibraciones de retemblado tienen lugar a una frecuencia cercana a la natural de vibración de la máquina y la pieza, pero no siempre, debido a la orientación del corte relativa a los modos de vibración [83].

La mejor manera de minimizar tanto las vibraciones forzadas como el retemblado es realizar un correcto diseño de la máquina herramienta, del anclaje y de los utillajes para que tengan alta rigidez dinámica $(2 k \zeta)$, especialmente en la dirección de las fuerzas principales de corte [84]. Tanto el aumento de la rigidez como del amortiguamiento son beneficiosos, al igual que la reducción de uno de estos parámetros compensado con un mayor aumento en el otro [78]. Frecuentemente un aumento de la rigidez se traduce en una reducción en el amortiguamiento, y puede ser más útil en esos casos incrementar el amortiguamiento para que aumente la rigidez dinámica de las estructuras [85,86]. Estos objetivos se pueden lograr mediante la simulación de máquinas herramientas con el método de los elementos finitos [87]. En algunos trabajos se calculan las funciones de 
respuesta en frecuencia del sistema de sujeción de herramienta [88]. Esto permite mejorar la rigidez dinámica del ensamblaje husillo-cojinete-herramienta optimizando las posiciones del cojinete y el accionamiento del motor a lo largo del eje del husillo.

Se puede evitar la influencia de las vibraciones en el acabado superficial estableciendo la frecuencia de giro de la herramienta como una fracción entera de la frecuencia natural del sistema herramienta-pieza, ya que en ese caso la deflexión de la herramienta sería la misma para la posición angular de la herramienta en la que genera la superficie. De este fenómeno se deduce la importancia de conocer los parámetros dinámicos del proceso para controlar la calidad del mecanizado.

\subsubsection{Control adaptativo}

El control adaptativo consiste en la regulación de un proceso que tiene cambio en dos velocidades o escalas de tiempo: el cambio en el sistema y el cambio en los parámetros [89]. Hay, por tanto, dos reguladores: uno del sistema y otro de los parámetros del regulador del sistema. El control adaptativo es conveniente cuando el punto de funcionamiento cambia sensiblemente, como ocurre en el proceso de microfresado cuando las fuerzas de corte se ven afectadas por un aumento significativo debido a la proximidad de la rotura. También varían de manera importante las fuerzas de corte cuando la herramienta cambia sus condiciones de corte, como la profundidad de corte, el avance por diente o la velocidad.

Se han realizado varios estudios de implantación del Control Adaptativo en el proceso de fresado [90]. El trabajo indicado aborda el análisis de un sistema de Control Adaptativo de los picos de las fuerzas en fresado con herramientas esbeltas, de tal manera que las condiciones son similares a las que concurren en el microfresado, ya que existe igualmente alto peligro de rotura ante variaciones bruscas de las fuerzas. La dinámica del proceso de fresado en este trabajo es modelada como un sistema discreto de primer orden. Se lleva a cabo un diseño sistemático de un controlador adaptativo PID y de colocación de polos. Se compara la eficacia de los dos controladores utilizando simulaciones y ensayos experimentales. Se demuestra que cuando se identifican con precisión y se desacoplan las funciones de transferencia del avance por diente y del 
proceso de corte dependiente del tiempo los sistemas de Control Adaptativo pueden ser aplicados con éxito a los procesos.

Hay dos tipos de Control Adaptativo: con Optimización y con Restricciones [91].

Varios autores han estudiado la aplicación del Control Adaptativo con Restricciones al fresado. Aunque los estudios iniciales constituyen una innovación en la aplicación de la teoría de control a los procesos de mecanizado, los métodos que proponen se pueden criticar en dos aspectos. Uno es el modelado del proceso de corte y el otro es la consideración de un proceso de corte invariante con el tiempo.

Los primeros trabajos trataron el proceso de corte como un simple sistema con ganancia [92] o con ganancia y retardo [93]. El primer análisis fundamental de la influencia de los cambios en el avance en el proceso de corte fue realizado por [93]. Los autores muestran en dicho trabajo que las fuerzas de corte tardan en alcanzar la estabilidad el periodo correspondiente al paso de un diente tras el cambio en el avance. [92] utilizaron un Control Adaptativo con Restricciones en un proceso de fresado periférico que se modeló como un sistema con ganancia y el retardo correspondiente a un periodo muestral.

Una de las principales aplicaciones de un modelo de fuerzas del microfresado es la utilización de un control adaptativo, que se compone de varios bloques, como se indica en la figura 2.9. Uno de estos bloques corresponde a la función de transferencia del proceso, que proporciona el valor de las fuerzas de corte para los diferentes parámetros de corte introducidos [90].

Las operaciones de microfresado tienen que ser desarrolladas de manera muy cuidadosa en cuanto al control y parámetros de corte para evitar vibraciones y otros efectos indeseables. Las pequeñas dimensiones de la herramienta hacen que a veces el desgaste, e incluso la rotura, no se detecten con facilidad y que por tanto, incluso horas de trabajo resulten desperdiciadas. 


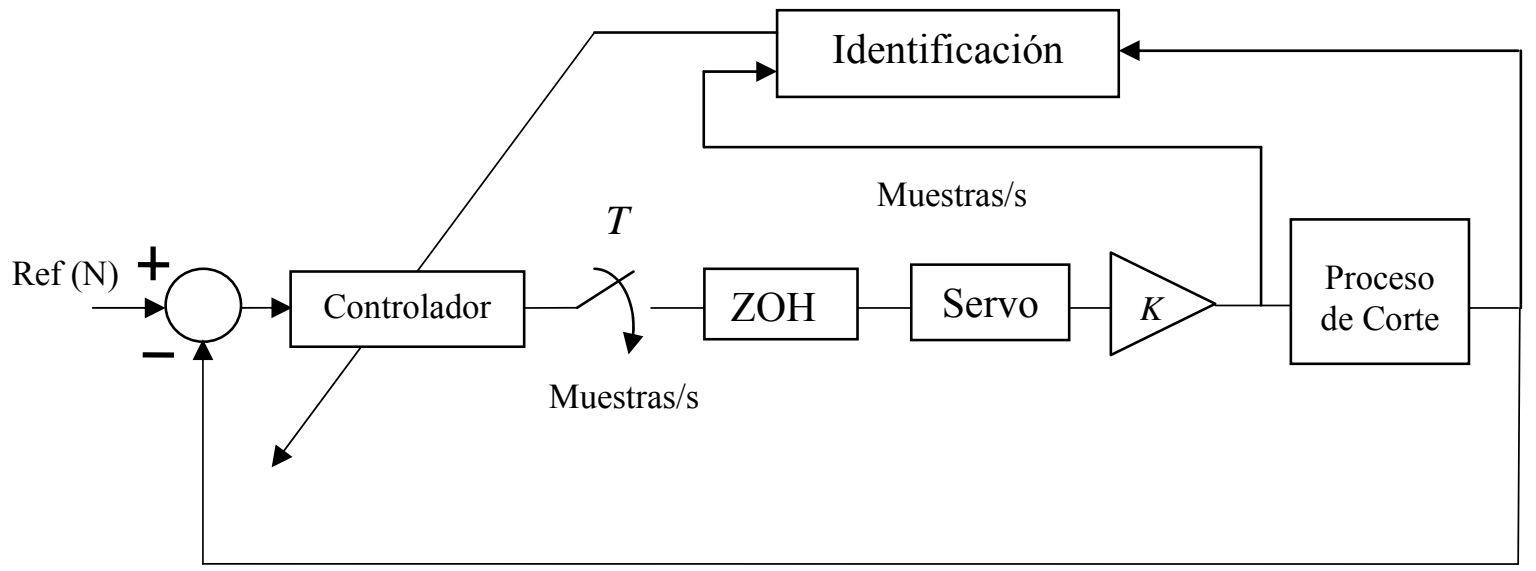

Figura 2.9. Esquema elemental de un sistema de control adaptativo

Es por ello que en este tipo de procesos de microfresado adquiere especial importancia el control dinámico del proceso en tiempo real. En las máquinas herramienta $\mathrm{CNC}$ convencionales, los parámetros de operación tales como velocidad de corte, velocidad de avance y profundidad de pasada se programan fuera de línea, sin la posibilidad de realizar ajustes. Sin la posibilidad de hacer estos ajustes en línea de forma dinámica, no es posible alcanzar de forma eficaz y eficiente el ritmo de producción óptimo (o lo que es lo mismo, la velocidad de arranque de material óptima). En los sistemas de fabricación integrados por ordenador modernos, es aceptado el hecho que la reducción en la denominada Efectividad Global del Equipo (OEE) se debe en parte a problemas relacionados con la herramienta, ya sea relacionados con la rotura de herramienta o con su desgaste [94]. Las herramientas desgastadas directamente reducen la capacidad del proceso y por tanto su productividad. Y esto es especialmente importante en el microfresado. 


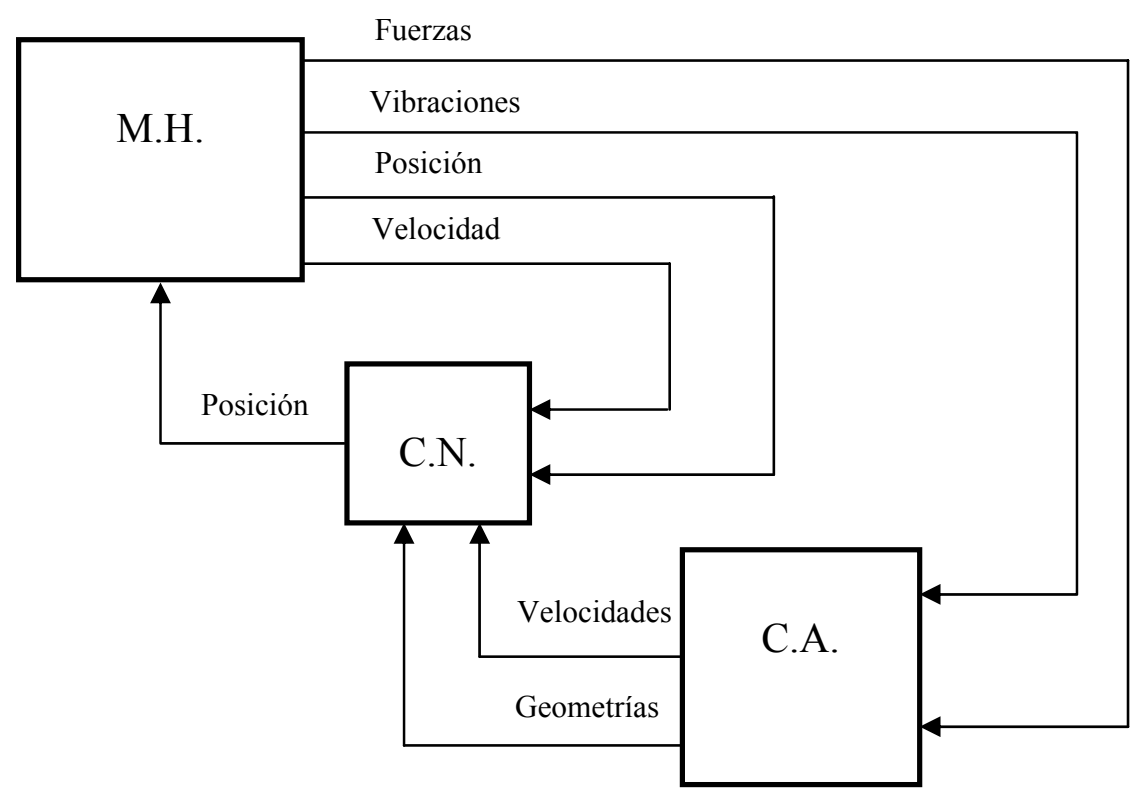

Figura 2.10. Esquema de elementos necesarios para un sistema de control adaptativo

Una solución a este problema consiste en realizar ajustes en tiempo real de los parámetros de operación, basándose para ello en información procedente de sensores colocados en la máquina, es decir, implementar un sistema de control adaptativo según se indica en la figura 2.10 [95][96]. Es necesario, por tanto, utilizar modelos que se adapten al cambio del proceso, como la progresión del desgaste de la herramienta, y por tanto también es necesario sistemas de medida en proceso que realimenten al control información sobre el estado de desgaste de la herramienta. Para ello se utilizarán los modelos analítico y empírico desarrollados en los pasos previos.

Se han desarrollado numerosos sistemas de monitorización de la herramienta de corte, con distinto grado de implantación en el entorno de producción [97][98], de forma que se pueda obtener el estado de desgaste de la herramienta sin necesidad de interrumpir el proceso de mecanizado, aspecto vital en la producción. La mayoría de los sistemas de monitorización de las condiciones de corte se basan en tres elementos principales: sensores; extracción de características y toma de decisiones.

Respecto a los sensores utilizados para la monitorización del estado de la herramienta, existen numerosos sistemas. Los de aplicación más práctica utilizan medidas indirectas sobre el funcionamiento del proceso, asociadas con la herramienta más que propiedades 
directamente medidas en la herramienta [98]. Las medidas indirectas son más fáciles de obtener. Todas ellas se basan en el hecho de que un incremento del área de contacto entre la herramienta y la pieza, debido al desgaste, produce un cambio en los parámetros del proceso, léase fuerzas de corte, temperatura, emisiones acústicas o vibraciones, siendo por tanto el objetivo la correlación de estas variables de proceso con el desgaste de la herramienta [99].

El análisis de vibraciones es un método aún poco robusto, debido a la dificultad para separar las señales procedentes de la vibración propia del funcionamiento de la máquina, de las debidas al desgaste de la herramienta, sin embargo, en los últimos trabajos están consiguiendo resultados muy interesantes [100].

En los últimos años, y debido a las limitaciones que presentan todos estos métodos en determinadas condiciones, se han empezado a implantar los sistemas de monitorización multi-sensor [101]. Estos sistemas compensan las limitaciones de unos sensores en determinados rangos con las ventajas de los otros, obteniendo resultados más fiables sobre el estado de la herramienta. Además, el desarrollo de los sistemas de adquisición de datos y de las capacidades de computación disponibles ha permitido poder trabajar con conjuntos de datos muy elevados procedentes de múltiples sensores. Sin embargo, dada la complejidad en la interpretación de las múltiples señales obtenidas, es preciso recurrir a técnicas como las redes neuronales [97].

Los trabajos de análisis de Control Adaptativo muestran la importancia de la utilización de un modelo preciso de predicción de fuerzas de corte para estimar correctamente la influencia de la variación de parámetros en el resultado del proceso. De ello se deduce la importancia que tiene la introducción de un nuevo modelo analítico de fuerzas de corte para ser aplicado en un sistema preciso de Control Adaptativo del proceso de microfresado como se explicará en el capítulo 9. 
ANÁLISIS DE LOS FACTORES DE INFLUENCIA EN EL PROCESO

\subsection{Fresado en condiciones ideales}

Antes de desarrollar el modelo para un caso real es conveniente observar las expresiones de las fuerzas que se obtienen para un caso ideal, es decir, aquél en el que no existe excentricidad ni deflexión de la herramienta. Este tipo de fresado no se da en la realidad, y por tanto, no sirve para realizar predicciones fiables. Sin embargo, este modelo proporciona tendencias válidas de los valores medios de las fuerzas para distintos valores de los parámetros. Por otro lado, puede servir para la estimación de algunos parámetros del modelo real, como se verá más adelante.

El modelo de fuerzas en el fresado con dientes helicoidales se establece en [35], considerando una constante de proporcionalidad fija entre la fuerza tangencial y la radial. En este trabajo se utilizará una constante de proporcionalidad dependiente del material de herramienta y pieza para adaptarse mejor a la variedad de condiciones que pueden darse en el proceso. Como se verá más adelante, la estimación de este valor resultará ser diferente para cada combinación de condiciones de corte.

El modelo utiliza una división del filo en discos diferenciales, cada uno de ellos situado a una altura $z$, como se indica en la figura 3.1. 


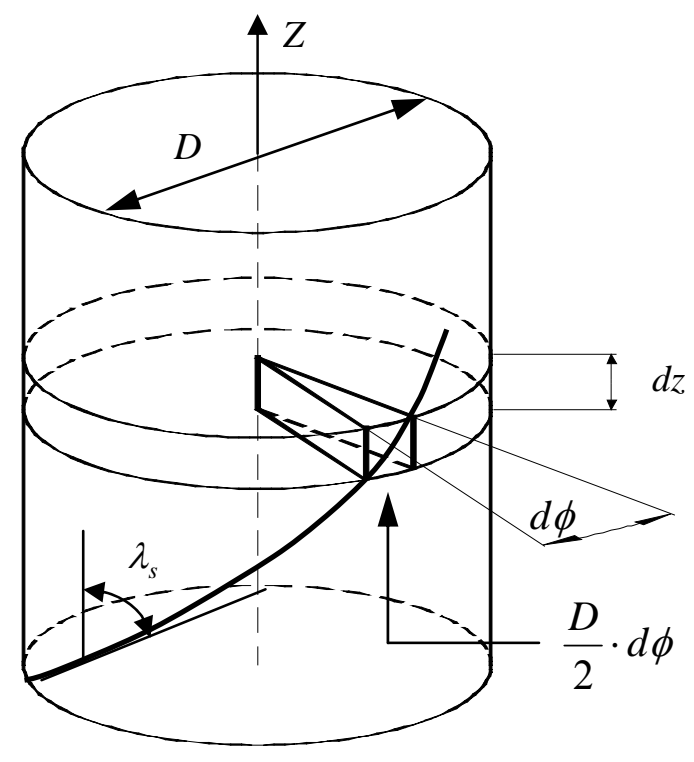

Figura 3.1. División del filo en elementos diferenciales

El espesor de la viruta se utilizará para calcular los valores de las fuerzas de corte, que dependerán de la geometría del proceso y de los parámetros del corte como la tensión dinámica de cizalladura, el ángulo de deslizamiento y el coeficiente de rozamiento [7]. Todos estos parámetros se pueden englobar en una constante [15][42]. Por otro lado, debido al redondeo del radio del filo existirá una fuerza de recalcado que aparecerá incluso cuando no se arranque viruta por el efecto tamaño Esta fuerza será constante para cualquier espesor de viruta. Suponiendo un filo vertical la fuerza sobre un diferencial de filo se puede expresar como suma de dos términos [41] expresándose de la siguiente manera:

$$
\begin{aligned}
& d F_{t}=\left(K_{t e}+K_{t c} \cdot t_{c}\right) \cdot d z \\
& d F_{r}=\left(K_{r e}+K_{r c} \cdot t_{c}\right) \cdot d z
\end{aligned}
$$

Las constantes presentes dependen a su vez del espesor de viruta, según se señala en [71], donde se expresa la presión de corte de la siguiente manera:

$$
K=k_{s} \cdot t_{c}^{m}
$$


Sin embargo, debido a que tanto el espesor de viruta como su variación son muy pequeños la presión específica de corte puede considerarse constante. Por tanto, las expresiones (3.1) y (3.2) pueden considerarse adecuadas para obtener las fuerzas del proceso.

Dado que en el fresado el corte no es ortogonal debido a la inclinación del filo, deberá adaptarse este modelo al corte oblicuo. Las fuerzas que actúan sobre el filo de la herramienta pueden esquematizarse en la figura 3.2, en la que se observa una componente axial debido a que el filo está inclinado verticalmente [102].

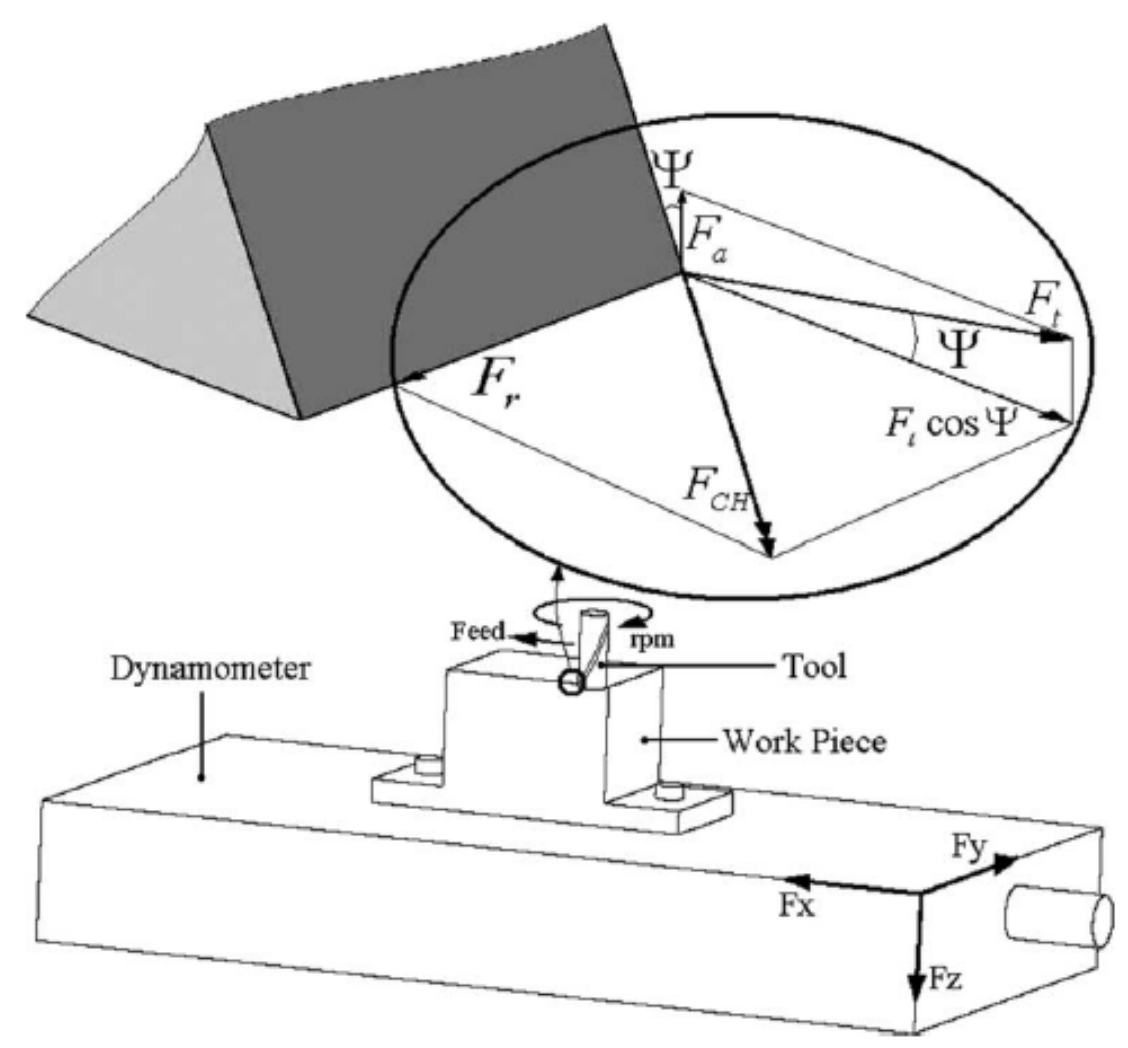

Figura 3.2. Esquema de las fuerzas en el filo de la herramienta

La presión de corte, por tanto, provocará una fuerza en dirección perpendicular al filo, que tendrá una componente vertical. No obstante, dado que por motivos de orden práctico las componentes más importantes de la fuerza son las horizontales debido a que son las que determinan la deflexión y por tanto la rotura de la herramienta, en este estudio sólo se tendrán en cuenta las componentes tangencial y radial. Las componentes 
tangencial horizontal y radial horizontal se pueden expresar en función de las componentes tangencial y radial y el ángulo de hélice:

$$
\begin{aligned}
& d F_{t h}=d F_{t} \cdot \cos \beta \\
& d F_{r h}=d F_{r} \cdot \cos \beta
\end{aligned}
$$

Sustituyendo aquí las expresiones (3.1) y (3.2) se obtiene:

$$
\begin{aligned}
& d F_{t h}=\left(K_{t e}+K_{t c} \cdot t_{c}\right) \cdot d l \cdot \cos \beta \\
& d F_{r h}=\left(K_{r e}+K_{r c} \cdot t_{c}\right) \cdot d l \cdot \cos \beta
\end{aligned}
$$

Donde $d l$ es el diferencial de longitud de filo. Este valor multiplicado por el coseno del ángulo de hélice $(\beta \circ \Psi)$ es precisamente el diferencial de altura $d z$, por lo que se puede reafirmar que las expresiones (3.1) y (3.2) proporcionan las componentes tangencial y radial de la fuerza del proceso, llamando $d F_{t}$ a $d F_{t h}$ y $d F_{r}$ a $d F_{r h}$, ya que las fuerzas que se considerarán en este estudio son sólo las horizontales.

\subsubsection{Expresiones para fresado en oposición}

Es necesario relacionar el espesor de viruta con las condiciones geométricas del fresado. Se considerarán dos tipos de fresado: en oposición y en concordancia, como se ha indicado anteriormente. La geometría del fresado en oposición está indicada en la Figura 3.3. 


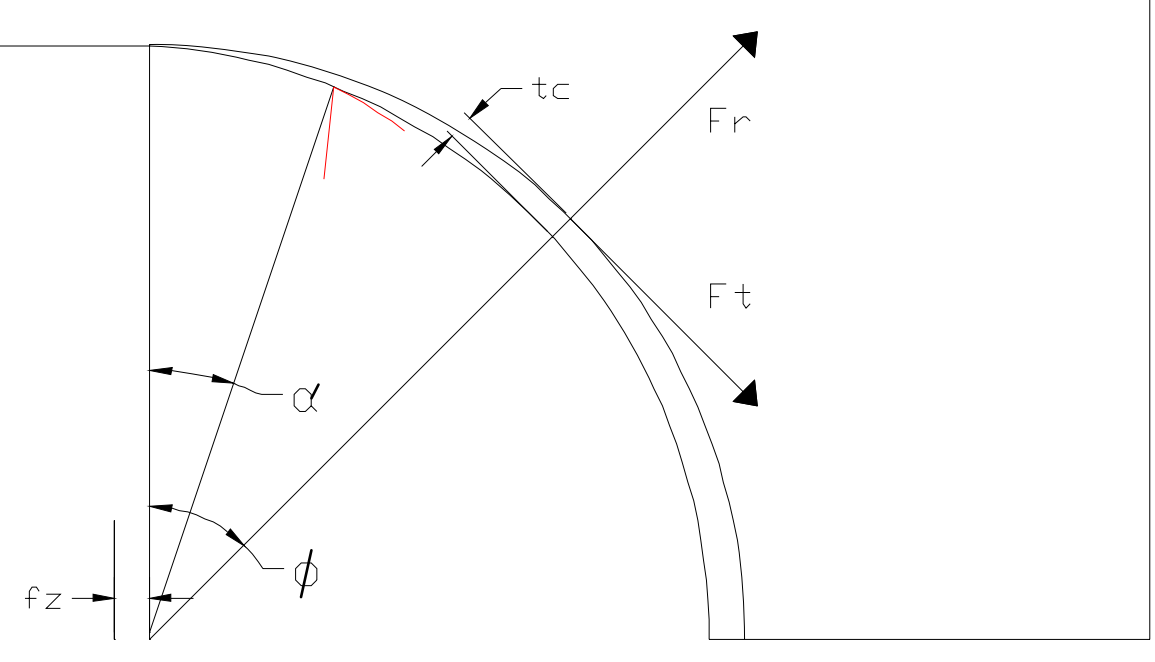

Figura 3.3. Geometría del fresado en oposición

Debido a que el filo de las fresas es helicoidal y cada punto tiene una posición angular distinta, el espesor de viruta que cada punto del filo está cortando será diferente y por tanto la fuerza de corte variará a lo largo del filo. Es necesario, por tanto, considerar el diferencial de fuerza en cada punto del filo. El diferencial de altura se puede expresar en función del diferencial de ángulo:

$$
d z=\frac{D}{2 \cdot \tan \beta} \cdot d \phi
$$

Donde $D$ es el diámetro de la herramienta. Una vez que se ha obtenido el diferencial de fuerza en cada punto del filo es preciso descomponerlo en coordenadas cartesianas para poder sumar las contribuciones de todos ellos. Observando la figura 3.3 se pueden deducir las expresiones que proporcionan los diferenciales de fuerza en las direcciones $x$ e $y$ a partir de las componentes tangencial y radial:

$$
\begin{gathered}
d F_{x}=d F_{t} \cdot \cos \phi+d F_{r} \cdot \sin \phi \\
d F_{y}=-d F_{t} \cdot \sin \phi+d F_{r} \cdot \cos \phi
\end{gathered}
$$


Siguiendo el procedimiento de [40] la proyección de las fuerzas se puede expresar en forma matricial:

$$
\left[\begin{array}{l}
d F_{x} \\
d F_{y}
\end{array}\right]=\left[\begin{array}{cc}
\cos \phi & \sin \phi \\
-\sin \phi & \cos \phi
\end{array}\right] \cdot\left[\begin{array}{l}
d F_{t} \\
d F_{r}
\end{array}\right]
$$

Sustituyendo las componentes tangencial y radial por su valor según las expresiones (3.1) y (3.2) se obtiene:

$$
\begin{aligned}
& d F_{x}=\frac{D}{2 \cdot \tan \beta} \cdot\left[K_{t e} \cdot \cos \phi+K_{r e} \cdot \sin \phi+\left(K_{t c} \cdot \cos \phi+K_{r c} \cdot \sin \phi\right) \cdot t_{c}\right] \cdot d \phi \\
& d F_{y}=\frac{D}{2 \cdot \tan \beta} \cdot\left[-K_{t e} \cdot \sin \phi+K_{r e} \cdot \cos \phi+\left(-K_{t c} \cdot \sin \phi+K_{r c} \cdot \cos \phi\right) \cdot t_{c}\right] \cdot d \phi
\end{aligned}
$$

Como se puede ver en la figura 3.3 hay dos ángulos que intervienen en la definición del espesor de viruta. El ángulo $\alpha$ es el que forma la punta del filo con la dirección de referencia, en este caso, la vertical. $\phi$ es el ángulo que forma en la vista horizontal el punto considerado del filo con la dirección de referencia. De acuerdo a este esquema se puede establecer la expresión para el espesor de viruta en cada punto del filo:

$$
t_{c}=f_{z} \cdot \sin \phi(3.14)
$$

Donde $f_{z}$ es el avance por diente de la herramienta. Sustituyendo la expresión (3.14) en las (3.12) y (3.13) se obtiene:

$$
\begin{gathered}
d F_{x}=\frac{D}{2 \cdot \tan \beta} \cdot\left[K_{t e} \cdot \cos \phi+K_{r e} \cdot \sin \phi+f_{z} \cdot\left(0.5 K_{t c} \cdot \sin 2 \phi+K_{r c} \cdot \sin ^{2} \phi\right)\right] \cdot d \phi \\
d F_{y}=\frac{D}{2 \cdot \tan \beta} \cdot\left[-K_{t e} \cdot \sin \phi+K_{r e} \cdot \cos \phi+f_{z} \cdot\left(-K_{t c} \cdot \sin ^{2} \phi+0.5 K_{r c} \cdot \sin 2 \phi\right)\right] \cdot d \phi
\end{gathered}
$$


Estas expresiones deben ser integradas a lo largo de todo el filo en contacto con la pieza para obtener la fuerza total en cada dirección. Para determinar los ángulos de comienzo y fin de contacto en cada periodo será necesario distinguir dos tipos de corte [35]. En las figuras 3.3 y 3.4 se esquematizan estos dos tipos de corte:

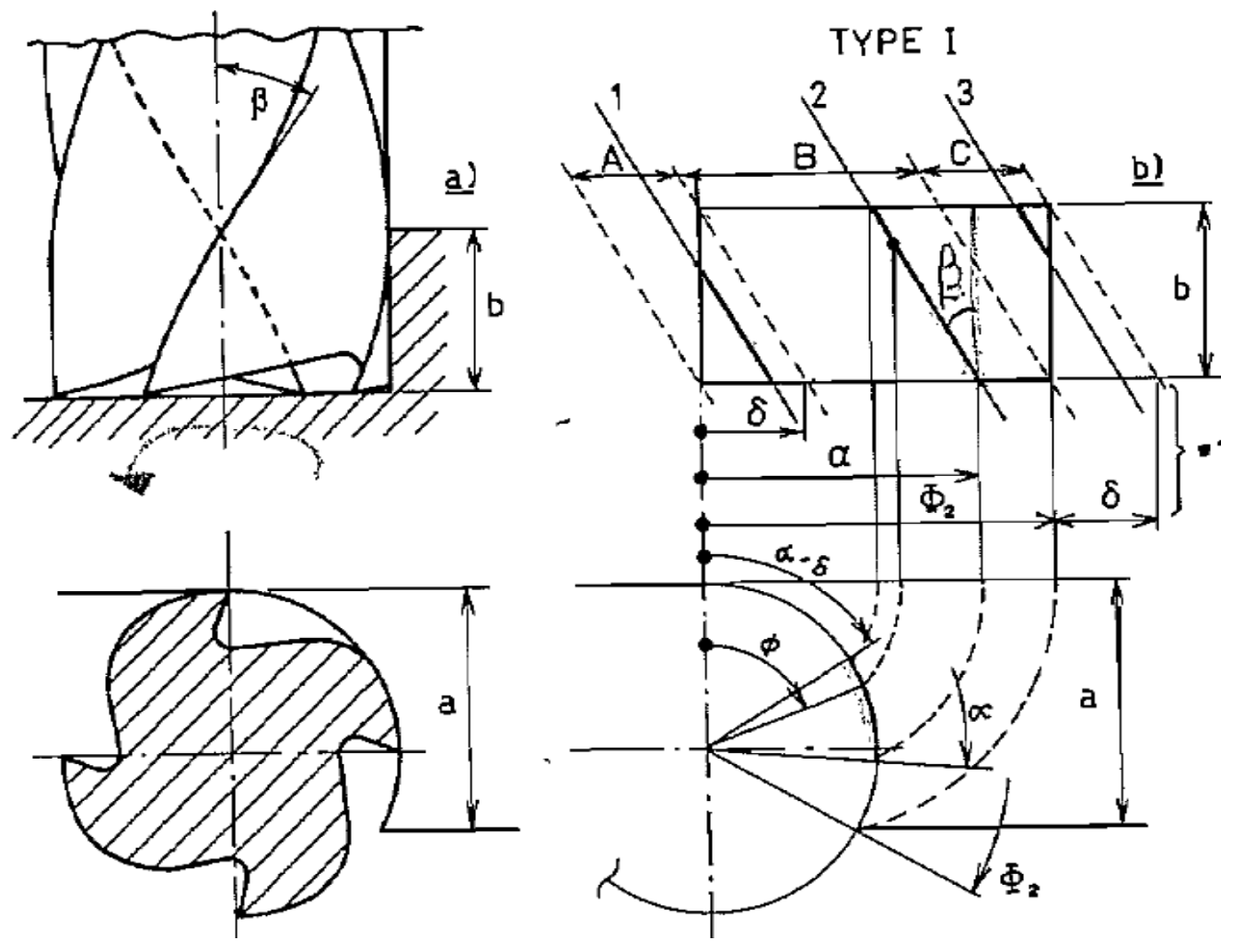

Figura 3.4. Fresado Tipo I 


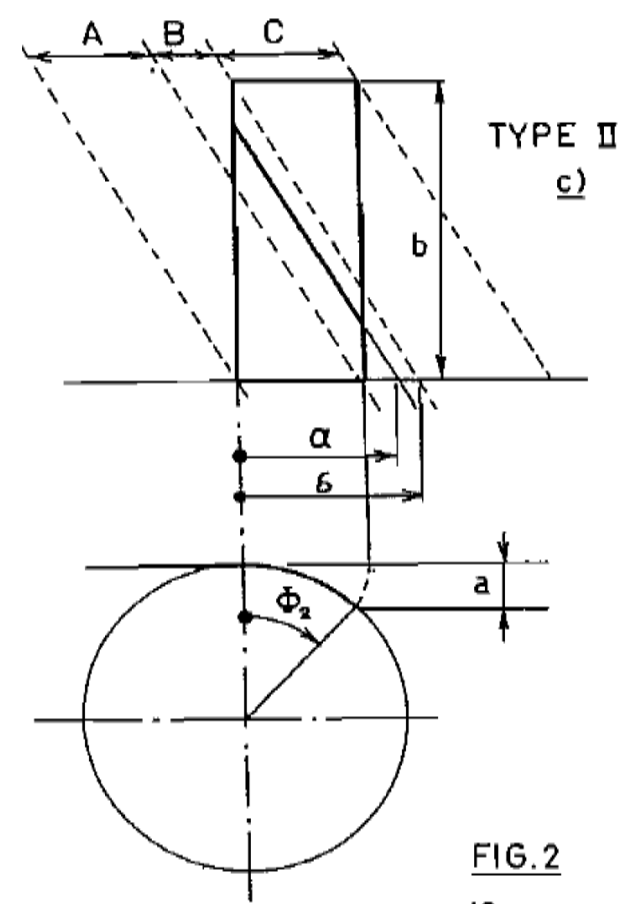

Figura 3.5. Fresado Tipo II

Como se aprecia en las figuras, $\delta$ es la distancia angular que cubre el filo desde su punta hasta la altura correspondiente a la profundidad axial de corte.

$$
\delta=\frac{2 \cdot a_{p} \cdot \tan \beta}{D}
$$

Donde $a_{p}$ es la profundidad axial de corte. $\Phi_{2}$ es el ángulo extremo de la zona de corte, es decir, aquél en el que el filo abandona la pieza. En el corte tipo I $\Phi_{2}$ es mayor que $\delta$, por lo que todo el filo entrará íntegramente en la zona de corte, mientras que en el corte tipo II, al ser $\Phi_{2}$ menor que $\delta$, sólo un tramo de filo estará en contacto con la pieza, ya que no cabe el filo entero. Esto hace que los límites de integración sean distintos.

En las figuras 3.4 y 3.5 también se distinguen 3 fases del corte para cada filo: A, B y C. En la primera el filo está entrando en la zona de corte y en la tercera está saliendo. La segunda fase es la intermedia entre las dos anteriores. 
A continuación se indican las expresiones que proporcionan las fuerzas para cada fase y tipo de corte.

Fase A:

$$
\begin{aligned}
& F_{x}=\frac{D}{2 \cdot \tan \beta} \cdot\left[\begin{array}{l}
K_{t e} \cdot \sin \alpha-K_{r e} \cdot \cos \alpha+K_{r e}+ \\
\frac{f_{z}}{4} \cdot\left(-K_{t c} \cdot \cos 2 \alpha+K_{t c}+2 K_{r c} \cdot \alpha-K_{r c} \cdot \sin 2 \alpha\right)
\end{array}\right] \\
& F_{y}=\frac{D}{2 \cdot \tan \beta} \cdot\left[\begin{array}{l}
K_{t e} \cdot \cos \alpha+K_{r e} \cdot \sin \alpha-K_{t e}+ \\
\frac{f_{z}}{4} \cdot\left(-2 K_{t c} \cdot \alpha+K_{t c} \cdot \sin 2 \alpha-K_{r c} \cdot \cos 2 \alpha+K_{r c}\right)
\end{array}\right]
\end{aligned}
$$

Fase B, Tipo I:

$$
\begin{gathered}
F_{x}=\frac{D}{2 \cdot \tan \beta} \cdot\left[\begin{array}{l}
K_{t e} \cdot \sin \alpha-K_{t e} \cdot \sin (\alpha-\delta)-K_{r e} \cdot \cos \alpha+K_{r e} \cdot \cos (\alpha-\delta)+ \\
\frac{f_{z}}{4} \cdot\left(\begin{array}{l}
-K_{t c} \cdot \cos 2 \alpha+K_{t c} \cdot \cos 2(\alpha-\delta)+ \\
2 K_{r c} \cdot \delta-K_{r c} \cdot \sin 2 \alpha+K_{r c} \cdot \sin 2(\alpha-\delta)
\end{array}\right)
\end{array}\right] \\
F_{y}=\frac{D}{2 \cdot \tan \beta} \cdot\left[\begin{array}{l}
K_{t e} \cdot \cos \alpha-K_{t e} \cdot \cos (\alpha-\delta)+K_{r e} \cdot \sin \alpha-K_{r e} \cdot \sin (\alpha-\delta)+ \\
\frac{f_{z}}{4} \cdot\left(\begin{array}{l}
-2 K_{t c} \cdot \delta+K_{t c} \cdot \sin 2 \alpha-K_{t c} \cdot \sin 2(\alpha-\delta)- \\
K_{r c} \cdot \cos 2 \alpha+K_{r c} \cdot \cos 2(\alpha-\delta)
\end{array}\right)
\end{array}\right]
\end{gathered}
$$

Fase B, Tipo II:

$$
\begin{aligned}
& F_{x}=\frac{D}{2 \cdot \tan \beta} \cdot\left[\begin{array}{l}
K_{t e} \cdot \sin \Phi_{2}-K_{r e} \cdot \cos \Phi_{2}+K_{r e}+ \\
\frac{f_{z}}{4} \cdot\left(-K_{t c} \cdot \cos 2 \Phi_{2}+K_{t c}+2 K_{r c} \cdot \Phi_{2}-K_{r c} \cdot \sin 2 \Phi_{2}\right)
\end{array}\right] \\
& F_{y}=\frac{D}{2 \cdot \tan \beta} \cdot\left[\begin{array}{l}
K_{t e} \cdot \cos \Phi_{2}-K_{t e}+K_{r e} \cdot \sin \Phi_{2}+ \\
\frac{f_{z}}{4} \cdot\left(-2 K_{t c} \cdot \Phi_{2}+K_{t c} \cdot \sin 2 \Phi_{2}-K_{r c} \cdot \cos 2 \Phi_{2}+K_{r c}\right)
\end{array}\right]
\end{aligned}
$$

Fase C: 


$$
\begin{gathered}
F_{x}=\frac{D}{2 \cdot \tan \beta} \cdot\left[\begin{array}{l}
K_{t e} \cdot \sin \Phi_{2}-K_{t e} \cdot \sin (\alpha-\delta)-K_{r e} \cdot \cos \Phi_{2}+K_{r e} \cdot \cos (\alpha-\delta)+ \\
\frac{f_{z}}{4} \cdot\left(\begin{array}{l}
-K_{t c} \cdot \cos 2 \Phi_{2}+K_{t c} \cdot \cos 2(\alpha-\delta)+ \\
2 K_{r c} \cdot\left(\Phi_{2}-\alpha+\delta\right)-K_{r c} \cdot \sin 2 \Phi_{2}+K_{r c} \cdot \sin 2(\alpha-\delta)
\end{array}\right)
\end{array}\right] \\
F_{y}=\frac{D}{2 \cdot \tan \beta} \cdot\left[\begin{array}{l}
K_{t e} \cdot \cos \Phi_{2}-K_{t e} \cdot \cos (\alpha-\delta)+K_{r e} \cdot \sin \Phi_{2}-K_{r e} \cdot \sin (\alpha-\delta)+ \\
\frac{f_{z}}{4} \cdot\left(\begin{array}{l}
-2 K_{t c} \cdot\left(\Phi_{2}-\alpha+\delta\right)+K_{t c} \cdot \sin 2 \Phi_{2}-K_{t c} \cdot \sin 2(\alpha-\delta)- \\
K_{r c} \cdot \cos 2 \Phi_{2}+K_{r c} \cdot \cos 2(\alpha-\delta)
\end{array}\right)
\end{array}\right]
\end{gathered}
$$

\subsubsection{Expresiones para fresado en concordancia}

En el fresado en concordancia, las expresiones cambiarán porque la geometría es distinta. En la figura 3.6 se muestra esta geometría.

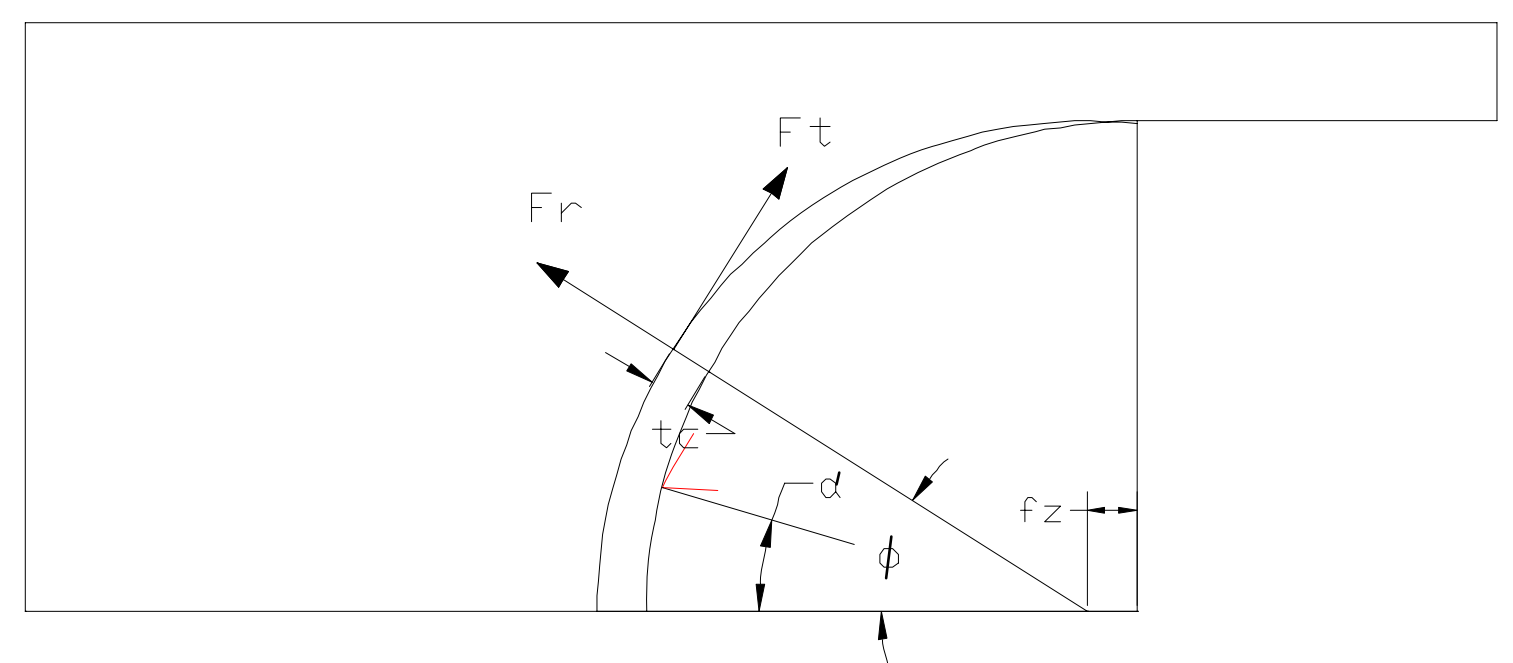

Figura 3.6. Geometría del fresado en concordancia

De acuerdo a esta geometría el espesor de viruta puede expresarse de la siguiente manera:

$$
t_{c}=f_{z} \cdot \cos \phi(3.26)
$$

La descomposición de las fuerzas será la siguiente: 


$$
\left[\begin{array}{l}
d F_{x} \\
d F_{y}
\end{array}\right]=\left[\begin{array}{cc}
\sin \phi & -\cos \phi \\
\cos \phi & \sin \phi
\end{array}\right] \cdot\left[\begin{array}{l}
d F_{t} \\
d F_{r}
\end{array}\right]
$$

Partiendo de las expresiones (3.1) y (3.2) se obtienen las componentes cartesianas de las fuerzas:

$$
\begin{aligned}
& d F_{x}=\frac{D}{2 \cdot \tan \beta} \cdot\left[K_{t e} \cdot \sin \phi-K_{r e} \cdot \cos \phi+\left(K_{t c} \cdot \sin \phi-K_{r c} \cdot \cos \phi\right) \cdot t_{c}\right] \cdot d \phi \\
& d F_{y}=\frac{D}{2 \cdot \tan \beta} \cdot\left[K_{t e} \cdot \cos \phi+K_{r e} \cdot \sin \phi+\left(K_{t c} \cdot \cos \phi+K_{r c} \cdot \sin \phi\right) \cdot t_{c}\right] \cdot d \phi
\end{aligned}
$$

Estas expresiones se integran entre los límites antes descritos para obtener las fuerzas totales, utilizando la fórmula del espesor de viruta ideal del fresado en concordancia proporcionada en la expresión (3.26):

Fase A:

$$
\begin{aligned}
& F_{x}=\frac{D}{2 \cdot \tan \beta} \cdot\left[\begin{array}{l}
-K_{t e} \cdot \cos \alpha+K_{t e}-K_{r e} \cdot \sin \alpha+ \\
\frac{f_{z}}{4} \cdot\left(-K_{t c} \cdot \cos 2 \alpha+K_{t c}-2 K_{r c} \cdot \alpha-K_{r c} \cdot \sin 2 \alpha\right)
\end{array}\right] \\
& F_{y}=\frac{D}{2 \cdot \tan \beta} \cdot\left[\begin{array}{l}
K_{t e} \cdot \sin \alpha-K_{r e} \cdot \cos \alpha+K_{r e}+ \\
\frac{f_{z}}{4} \cdot\left(2 K_{t c} \cdot \alpha+K_{t c} \cdot \sin 2 \alpha-K_{r c} \cdot \cos 2 \alpha+K_{r c}\right)
\end{array}\right]
\end{aligned}
$$

Fase B, Tipo I:

$$
F_{x}=\frac{D}{2 \cdot \tan \beta} \cdot\left[\begin{array}{l}
-K_{t e} \cdot \cos \alpha+K_{t e} \cdot \cos (\alpha-\delta)-K_{r e} \cdot \sin \alpha+K_{r e} \cdot \sin (\alpha-\delta)+ \\
\frac{f_{z}}{4} \cdot\left(\begin{array}{l}
-K_{t c} \cdot \cos 2 \alpha+K_{t c} \cdot \cos 2(\alpha-\delta)- \\
2 K_{r c} \cdot \delta-K_{r c} \cdot \sin 2 \alpha+K_{r c} \cdot \sin 2(\alpha-\delta)
\end{array}\right)
\end{array}\right]
$$




$$
F_{y}=\frac{D}{2 \cdot \tan \beta} \cdot\left[\begin{array}{l}
K_{t e} \cdot \sin \alpha-K_{t e} \cdot \sin (\alpha-\delta)-K_{r e} \cdot \cos \alpha+K_{r e} \cdot \cos (\alpha-\delta)+ \\
\frac{f_{z}}{4} \cdot\left(\begin{array}{l}
2 K_{t c} \cdot \delta+K_{t c} \cdot \sin 2 \alpha-K_{t c} \cdot \sin 2(\alpha-\delta)- \\
K_{r c} \cdot \cos 2 \alpha+K_{r c} \cdot \cos 2(\alpha-\delta)
\end{array}\right)
\end{array}\right]
$$

Fase B, Tipo II:

$$
\begin{aligned}
& F_{x}=\frac{D}{2 \cdot \tan \beta} \cdot\left[\begin{array}{l}
-K_{t e} \cdot \cos \Phi_{2}+K_{t e}-K_{r e} \cdot \sin \Phi_{2}+ \\
\frac{f_{z}}{4} \cdot\left(-K_{t c} \cdot \cos 2 \Phi_{2}+K_{t c}-2 K_{r c} \cdot \Phi_{2}-K_{r c} \cdot \sin 2 \Phi_{2}\right)
\end{array}\right] \\
& F_{y}=\frac{D}{2 \cdot \tan \beta} \cdot\left[\begin{array}{l}
K_{t e} \cdot \sin \Phi_{2}-K_{r e} \cdot \cos \Phi_{2}+K_{r e}+ \\
\frac{f_{z}}{4} \cdot\left(2 K_{t c} \cdot \Phi_{2}+K_{t c} \cdot \sin 2 \Phi_{2}-K_{r c} \cdot \cos 2 \Phi_{2}+K_{r c}\right)
\end{array}\right]
\end{aligned}
$$

Fase C:

$$
\begin{aligned}
& F_{x}=\frac{D}{2 \cdot \tan \beta} \cdot\left[\begin{array}{l}
-K_{t e} \cdot \cos \Phi_{2}+K_{t e} \cdot \cos (\alpha-\delta)- \\
K_{r e} \cdot \sin \Phi_{2}+K_{r e} \cdot \sin (\alpha-\delta)+ \\
\frac{f_{z}}{4} \cdot\left(\begin{array}{l}
-K_{t c} \cdot \cos 2 \Phi_{2}+K_{t c} \cdot \cos 2(\alpha-\delta)- \\
2 K_{r c} \cdot\left(\Phi_{2}-\alpha+\delta\right)-K_{r c} \cdot \sin 2 \Phi_{2}+K_{r c} \cdot \sin 2(\alpha-\delta)
\end{array}\right)
\end{array}\right] \\
& F_{y}=\frac{D}{2 \cdot \tan \beta} \cdot\left[\begin{array}{l}
K_{t e} \cdot \sin \Phi_{2}-K_{t e} \cdot \sin (\alpha-\delta)-K_{r e} \cdot \cos \Phi_{2}+K_{r e} \cdot \cos (\alpha-\delta)+ \\
\frac{f_{z}}{4} \cdot\left(\begin{array}{l}
2 K_{t c} \cdot\left(\Phi_{2}-\alpha+\delta\right)+K_{t c} \cdot \sin 2 \Phi_{2}- \\
K_{t c} \cdot \sin 2(\alpha-\delta)-K_{r c} \cdot \cos 2 \Phi_{2}+K_{r c} \cdot \cos 2(\alpha-\delta)
\end{array}\right)
\end{array}\right]
\end{aligned}
$$

\subsection{Influencia de la desviación excéntrica de la herramienta}

La desviación excéntrica o run-out tiene una gran influencia en el proceso de microfresado, ya que provoca una desviación del filo de la herramienta respecto a su posición teórica. En algunas máquinas herramienta este fenómeno no se puede evitar, debido a que existen asimetrías en el sistema de sujeción de herramienta. Este factor ha sido considerado en numerosos modelos de fuerzas en el fresado, como [103], [104] y 
[105]. También ha sido considerado explícitamente en el proceso de microfresado [68]. Siguiendo las consideraciones de [39] la desviación excéntrica de la herramienta puede esquematizarse según se indica en la Figura 6, donde se muestra el husillo junto con el ajuste de la herramienta. En la posición de la herramienta se combina el error de posición debido a la sujeción y los errores debidos a movimientos asíncronos del cojinete del husillo. Todo ello provoca una desviación de herramienta de entre 3 y 20 veces el espesor nominal de la viruta en el caso del fresado convencional. En el microfresado este valor puede reducirse hasta ser del orden del espesor de la viruta, pero en cualquier caso deberá ser tenido en cuenta para modelar con precisión las fuerzas.

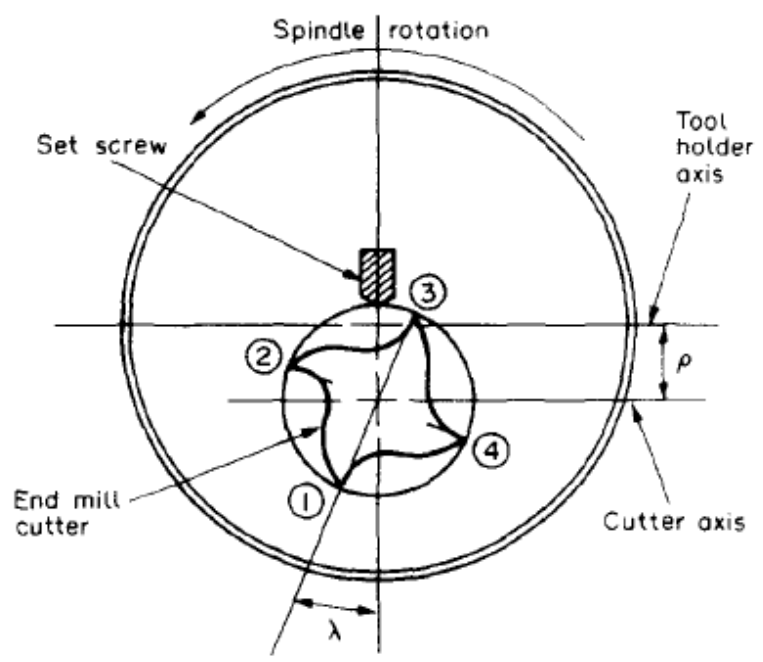

Figura 3.7. Desviación excéntrica debida al sistema de sujeción de herramienta

La desviación excéntrica provoca una distorsión en el espesor de viruta indeformada, que a su vez causa una variación de las fuerzas de corte, ya que son proporcionales al espesor de viruta. Esta desviación de la trayectoria de la herramienta será diferente dependiendo del diente que se considere. Por lo tanto, habrá variación entre la señal de fuerza de corte debida a un diente y a otro. Este efecto se puede observar en las medidas experimentales de las fuerzas, ya que las alturas de picos consecutivos son diferentes.

La desviación excéntrica de la herramienta puede causar también una falta de precisión en la superficie mecanizada, así como un incremento en su rugosidad superficial, debido a que variará la profundidad radial de corte de un diente a otro. 
La consideración matemática de la desviación excéntrica en el microfresado consistirá en añadir un término a la expresión del espesor de viruta, debido a la diferencia de radios de dos cortes consecutivos, tal como se hace en el fresado convencional [39]. La figura 3.8 muestra la geometría de dos cortes consecutivos del proceso separados una distancia igual al avance por diente, para el caso del fresado en oposición.

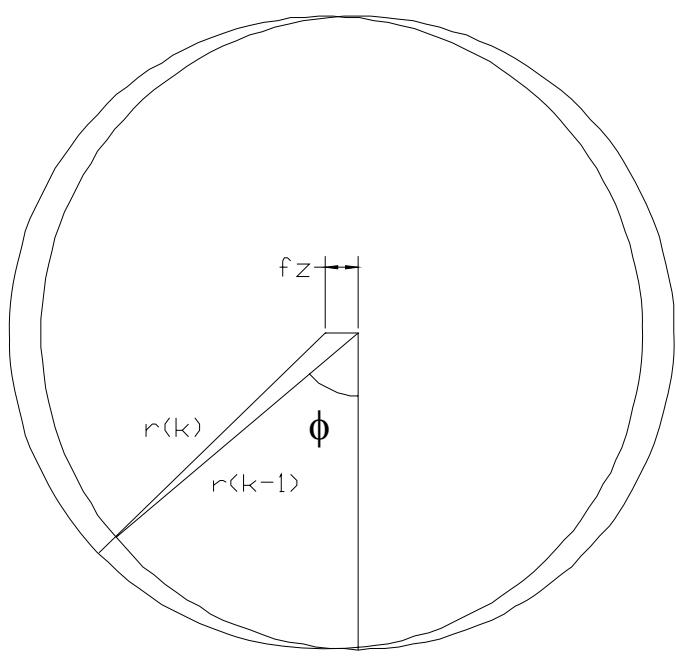

Figura 3.8. Geometría del fresado con desviación excéntrica

Según esta geometría, el espesor de viruta se puede expresar de la siguiente manera operaciones de fresado en oposición:

$$
t_{c}(k)=[r(k)-r(k-1)]+f_{z} \cdot \sin \phi
$$

Donde

$t_{c}(k)$ es el espesor de viruta

$r(k)$ es el radio de corte del filo considerado

$r(k-1)$ es el radio de corte del filo anterior

$f_{z}$ es el avance por diente

$\phi$ es el ángulo de posición respecto a la referencia del punto del filo considerado 
$r(k)$ y $r(k-1)$ son diferentes debido a la desviación excéntrica y pueden expresarse con la siguiente fórmula:

$$
\begin{gathered}
r(k)=\frac{D}{2}+\rho \cdot \cos [\Delta \phi-\lambda+(k-1) \cdot \pi] \\
k=1,2
\end{gathered}
$$

Donde:

$D$ es el diámetro de la fresa $\rho$ es el valor de la desviación excéntrica o run-out $\lambda$ es el ángulo que forma el filo principal con la dirección de máximo run-out $k=1,2$ según el filo considerado

$\Delta \phi$ es el ángulo horizontal que hay entre el extremo inferior del filo y el punto considerado

Por tanto, el espesor de viruta para el primer filo $(k=1)$ vendrá dado por la siguiente expresión:

$$
t_{c}(1)=f_{z} \cdot \sin \phi+\frac{D}{2}+\rho \cdot \cos (\Delta \phi-\lambda)-\frac{D}{2}-\rho \cdot \cos (\Delta \phi-\lambda+\pi)
$$

Simplificando:

$$
t_{c}(1)=f_{z} \cdot \sin \phi+2 \cdot \rho \cdot \cos (\Delta \phi-\lambda)
$$

De igual manera, para $k=2$ :

$$
t_{c}(2)=f_{z} \cdot \sin \phi-2 \cdot \rho \cdot \cos (\Delta \phi-\lambda)
$$

Estas expresiones son válidas también para el fresado en concordancia si se sustituye el seno por el coseno en el término del espesor ideal de viruta. 
Por tanto, el efecto de la desviación excéntrica depende sólo del punto del filo que se considere y no de la posición angular de la fresa. Si se quisiera obtener la expresión para el proceso de fresado en concordancia habría que sustituir en el primer término de las expresiones (3.41) y (3.42) el seno por el coseno.

Con estas expresiones se puede calcular el espesor de la viruta en ausencia de otros factores de influencia en el proceso como la deflexión de herramienta. Dado que en el modelo propuesto se considerará este factor, las expresiones anteriormente desarrolladas se tomarán únicamente como base para el desarrollo del modelo.

\subsection{Corte asimétrico}

La desviación excéntrica también provoca que el filo de la herramienta no comience a cortar en el punto de ángulo 0 sino en un ángulo distinto en el fresado en oposición. El ángulo de salida del filo experimenta una variación similar en el fresado en concordancia. Debido a que la desviación de la herramienta variará con la posición angular de ésta, el ángulo de entrada y salida será distinto para cada altura considerada. Por tanto, a la hora de determinar las fuerzas deberá realizarse un cálculo de los ángulos de entrada y salida para cada diente en cada altura.

La diferencia de ángulos de entrada y de salida para cada diente provoca que exista un rango de ángulos en el que sólo corta uno de los dientes. Este tipo de corte se llama asimétrico porque es "desequilibrado", es decir, no está influenciado por la acción de los dos dientes. En el resto del recorrido, actuarán los dos dientes y realizarán por tanto un corte simétrico. El corte asimétrico no está influenciado por el run-out porque existirá la misma desviación en dos cortes consecutivos por estar realizadas por el mismo diente y por tanto el espesor de viruta no se verá afectado. Siguiendo el modelo convencional del fresado [7] la expresión para el cálculo del espesor de viruta en corte asimétrico será la siguiente para fresado en oposición:

$$
t_{c}(1)=2 \cdot f_{z} \cdot \sin \phi
$$


Se ha tomado 1 como valor de $k$ porque se considera que es el primer diente el que realiza corte asimétrico.

\section{Fresado en oposición}

Las fuerzas radial y tangencial se pueden obtener adaptando las expresiones (3.1) y (3.2) a este tipo de corte:

$$
\begin{aligned}
& d F_{t}(1)=\frac{D}{2 \cdot \tan \beta} \cdot\left(K_{t e}+2 K_{t c} \cdot f_{z} \cdot \sin \phi\right) \cdot d \phi(3.44) \\
& d F_{r}(1)=\frac{D}{2 \cdot \tan \beta} \cdot\left(K_{r e}+2 K_{r c} \cdot f_{z} \cdot \sin \phi\right) \cdot d \phi(3.45)
\end{aligned}
$$

En el fresado en oposición el corte asimétrico ocurrirá en la zona inicial del corte de cada diente, ya que es ahí donde los valores del espesor de viruta son más reducidos y por tanto donde el ángulo de entrada será más sensible a las variaciones debidas a la desviación excéntrica. En la zona de salida, en cambio, según se puede ver en la figura 2 el espesor de viruta es mayor y no estará sujeto a estas variaciones por lo general, por lo que el ángulo de salida será aquél en el que la herramienta forzosamente deja a la pieza, es decir $\Phi_{2}$.

Las fuerzas tangencial y radial se proyectan sobre las direcciones cartesianas como se hizo en la sección 1:

$$
\left[\begin{array}{l}
d F_{x}(1) \\
d F_{y}(1)
\end{array}\right]=\left[\begin{array}{cc}
\cos \phi & \sin \phi \\
-\sin \phi & \cos \phi
\end{array}\right] \cdot\left[\begin{array}{l}
d F_{t}(1) \\
d F_{r}(1)
\end{array}\right]
$$

De aquí se obtienen las expresiones para los diferenciales de fuerza:

$$
d F_{x}(1)=\frac{D}{2 \cdot \tan \beta} \cdot\left[\begin{array}{l}
K_{t e} \cdot \cos \phi+K_{r e} \cdot \sin \phi+ \\
f_{z} \cdot\left(K_{t c} \cdot \sin 2 \phi+2 K_{r c} \cdot \sin ^{2} \phi\right)
\end{array}\right] \cdot d \phi
$$




$$
d F_{y}(1)=\frac{D}{2 \cdot \tan \beta} \cdot\left[\begin{array}{l}
-K_{t e} \cdot \sin \phi+K_{r e} \cdot \cos \phi+ \\
f_{z} \cdot\left(-2 K_{t c} \cdot \sin ^{2} \phi+K_{r c} \cdot \sin 2 \phi\right)
\end{array}\right] \cdot d \phi
$$

Estas expresiones han de ser integradas entre los dos ángulos entre los cuales exista corte asimétrico. El ángulo inicial no será 0 , ya que el filo sólo empieza a cortar cuando el espesor de viruta llega a un cierto valor mínimo debido al efecto, tamaño, según se explica en el capítulo siguiente. El ángulo final será aquél en el que el espesor de viruta llegue al valor mínimo como para que el segundo filo empiece a cortar. En ese momento dará comienzo el corte simétrico, es decir, la región en la cual cortan los dos filos.

Suponiendo que los ángulos límite son $\phi_{1} \mathrm{y} \phi_{2}$ las fuerzas vienen dadas por las siguientes expresiones:

$$
\begin{aligned}
& F_{x}(1)=\frac{D}{2 \cdot \tan \beta} \cdot\left[\begin{array}{l}
K_{t e} \cdot\left(\sin \phi_{2}-\sin \phi_{1}\right)-K_{r e} \cdot\left(\cos \phi_{2}-\cos \phi_{1}\right)+ \\
\frac{f_{z}}{2} \cdot\left[\begin{array}{l}
-K_{t c} \cdot\left(\cos 2 \phi_{2}-\cos 2 \phi_{1}\right)+2 K_{r c} \cdot\left(\phi_{2}-\phi_{1}\right)- \\
K_{r c} \cdot\left(\sin 2 \phi_{2}-\sin 2 \phi_{1}\right)
\end{array}\right]
\end{array}\right] \\
& F_{y}(1)=\frac{D}{2 \cdot \tan \beta} \cdot\left[\begin{array}{l}
K_{t e} \cdot\left(\cos \phi_{2}-\cos \phi_{1}\right)+K_{r e} \cdot\left(\sin \phi_{2}-\sin \phi_{1}\right)+ \\
\frac{f_{z}}{2} \cdot\left[\begin{array}{l}
-2 K_{t c} \cdot\left(\phi_{2}-\phi_{1}\right)+K_{t c} \cdot\left(\sin 2 \phi_{2}-\sin 2 \phi_{1}\right)- \\
K_{r c} \cdot\left(\cos 2 \phi_{2}-\cos 2 \phi_{1}\right)
\end{array}\right]
\end{array}\right]
\end{aligned}
$$

\section{Fresado en concordancia}

Las expresiones de la fuerza en corte asimétrico para este tipo de fresado se pueden obtener sustituyendo el seno por el coseno en las expresiones (3.44) y (3.45):

$$
\begin{aligned}
& d F_{t}(1)=\frac{D}{2 \cdot \tan \beta} \cdot\left(K_{t e}+2 K_{t c} \cdot f_{z} \cdot \cos \phi\right) \cdot d \phi \\
& d F_{r}(1)=\frac{D}{2 \cdot \tan \beta} \cdot\left(K_{r e}+2 K_{r c} \cdot f_{z} \cdot \cos \phi\right) \cdot d \phi
\end{aligned}
$$

La descomposición en coordenadas cartesianas se deducirá de la expresión (3.27): 


$$
\left[\begin{array}{l}
d F_{x}(1) \\
d F_{y}(1)
\end{array}\right]=\left[\begin{array}{cc}
\sin \phi & -\cos \phi \\
\cos \phi & \sin \phi
\end{array}\right] \cdot\left[\begin{array}{l}
d F_{t}(1) \\
d F_{r}(1)
\end{array}\right]
$$

Que dará lugar a las siguientes expresiones para los diferenciales de fuerza:

$$
\begin{aligned}
& d F_{x}(1)=\frac{D}{2 \cdot \tan \beta} \cdot\left[\begin{array}{l}
K_{t e} \cdot \sin \phi-K_{r e} \cdot \cos \phi+ \\
f_{z} \cdot\left(K_{t c} \cdot \sin 2 \phi-K_{r c}-K_{r c} \cdot \cos 2 \phi\right)
\end{array}\right] \cdot d \phi \\
& d F_{y}(1)=\frac{D}{2 \cdot \tan \beta} \cdot\left[\begin{array}{l}
K_{t e} \cdot \cos \phi+K_{r e} \cdot \sin \phi+ \\
f_{z} \cdot\left(K_{t c}+K_{t c} \cdot \cos 2 \phi+K_{r c} \cdot \sin 2 \phi\right)
\end{array}\right] \cdot d \phi
\end{aligned}
$$

Estas expresiones han de ser integradas entre los límites de la zona de corte asimétrico en fresado en concordancia, que estará cercana al ángulo de salida $\Phi_{2}$, ya que al haber en esa zona pequeños espesores de viruta, es fácil que el de uno de los dientes sea nulo por la distorsión que provoca la desviación excéntrica. Llamando $\phi_{1}$ y $\phi_{2}$ a los límites de integración se obtiene:

$$
\begin{aligned}
& F_{x}(1)=\frac{D}{2 \cdot \tan \beta} \cdot\left[\begin{array}{l}
-K_{t e} \cdot\left(\cos \phi_{2}-\cos \phi_{1}\right)-K_{r e} \cdot\left(\sin \phi_{2}-\sin \phi_{1}\right)+ \\
\frac{f_{z}}{2} \cdot\left[\begin{array}{l}
-K_{t c} \cdot\left(\cos 2 \phi_{2}-\cos 2 \phi_{1}\right)-2 K_{r c} \cdot\left(\phi_{2}-\phi_{1}\right)- \\
K_{r c} \cdot\left(\sin 2 \phi_{2}-\sin 2 \phi_{1}\right)
\end{array}\right]
\end{array}\right] \\
& F_{y}(1)=\frac{D}{2 \cdot \tan \beta} \cdot\left[\begin{array}{l}
K_{t e} \cdot\left(\sin \phi_{2}-\sin \phi_{1}\right)-K_{r e} \cdot\left(\cos \phi_{2}-\cos \phi_{1}\right)+ \\
\frac{f_{z}}{2} \cdot\left[\begin{array}{l}
2 K_{t c} \cdot\left(\phi_{2}-\phi_{1}\right)+K_{t c} \cdot\left(\sin 2 \phi_{2}-\sin 2 \phi_{1}\right)- \\
K_{r c} \cdot\left(\cos 2 \phi_{2}-\cos 2 \phi_{1}\right)
\end{array}\right]
\end{array}\right]
\end{aligned}
$$

\subsection{Influencia de la deflexión de herramienta}

Partiendo de la deflexión se puede caracterizar la falta de precisión que este fenómeno provoca en la pieza mecanizada. La reducción en la precisión dependerá del valor de la fuerza en la dirección perpendicular a la superficie mecanizada, ya que el desplazamiento de la herramienta en esa dirección es el que afectará a la posición de la 
superficie. Debido al perfil lineal de la deformación, la influencia de la deflexión de la herramienta en la precisión de la superficie será máxima en su parte inferior.

La distorsión de las fuerzas de corte puede ser estudiada determinando el cambio que provoca la deflexión en el espesor de la viruta. El espesor de viruta depende de la posición de la herramienta en dos cortes consecutivos. Por lo tanto, si no hubiera desviación excéntrica, el espesor de viruta no se vería afectado por la deflexión, ya que ésta provocaría la misma variación de posición en un diente que en otro y consecuentemente no habría distorsión en las fuerzas de corte. Por tanto, la deflexión de herramienta debe ser considerada junto al run-out para observar su influencia.

Si existe desviación excéntrica en la herramienta, los dientes sufren un desplazamiento respecto a su posición teórica, uno hacia delante y otro hacia atrás. Esto hace que el espesor de viruta sea mayor en uno de los dientes que en otro. Por tanto, las fuerzas serán superiores en el diente que más material corta. Sin embargo, la deflexión provoca un desplazamiento hacia atrás superior en este diente que en el otro influenciando el espesor de viruta a su vez. De esta manera, la deflexión constituye una especie de amortiguamiento del efecto de la desviación excéntrica.

Para obtener los valores de las fuerzas se deberá deducir el espesor de viruta en cada posición de la herramienta. Para ello, deberán tenerse en cuenta las fuerzas que actúan en un diente y en el anterior, dado que éstas determinarán la posición de los dos filos consecutivos, que a su vez fijará el valor del espesor de viruta. En las siguientes secciones se analizará este fenómeno en varias operaciones de fresado obteniéndose una predicción completa de las fuerzas del proceso.

A continuación se desarrollará el modelo de deflexión de herramienta propuesto, con el objeto de obtener una expresión matemática de la desviación que las fuerzas de corte provocan en la herramienta. Esta expresión será aplicada en el siguiente capítulo en la obtención del espesor de viruta para los distintos procesos de fresado.

En primer lugar, se expondrá la formulación del modelo asimilando la deformación de herramienta a una función lineal dependiente de la distancia del punto considerado al extremo de la herramienta. Posteriormente se obtendrán las constantes que intervienen 
en la función de la deformación partiendo de los conceptos que la elasticidad y resistencia de materiales proporcionan para la deformación de barras sometidas a tensión.

\subsubsection{Formulación del modelo}

Las herramientas usadas en microfresado tienen un pequeño diámetro de mango que provoca una escasa resistencia a la flexión, por lo que se deforman fácilmente por la acción de las fuerzas del proceso. Esto hace que exista una cierta desviación de la herramienta respecto a su posición teórica. De este fenómeno se seguirán dos efectos principales: la falta de precisión en la pieza mecanizada [106] y la distorsión de las fuerzas de corte. La deflexión de herramienta ya ha sido considerada en algunos modelos de fresado convencional [37] pero no ha sido todavía incorporada al cálculo de las fuerzas.

Para el cálculo de la deflexión de herramienta se considerará que es un elemento elástico sujeto mediante un empotramiento en su parte superior y sometido a una fuerza uniformemente distribuida a lo largo del tramo final, correspondiente a la profundidad axial de corte $a_{p}$, según el esquema que se indica en la figura 3.9.

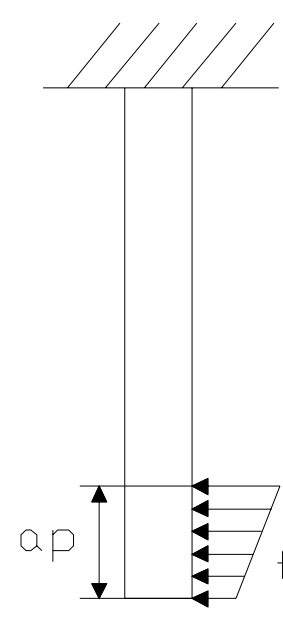

Figura 3.9. Fuerza distribuida sobre la herramienta 
Esta fuerza distribuida puede concentrarse en una fuerza puntual cuya magnitud sea la suma total de las fuerzas en cada punto y cuya posición sea tal que provoque el mismo momento flector que la fuerza distribuida, según se indica en la figura 3.10.

La fuerza situada en esa posición provocará un perfil de deformación lineal en la herramienta [107], del que el valor máximo corresponderá al extremo inferior. La siguiente expresión, tomada del análisis elástico de una viga en las condiciones indicadas, proporciona la desviación máxima que tiene lugar en ella bajo la acción de una fuerza $F_{T}$ :

$$
d_{\max }=\frac{(L-a)^{2} \cdot(2 L+a)}{6 \cdot E \cdot I} \cdot F_{T}
$$

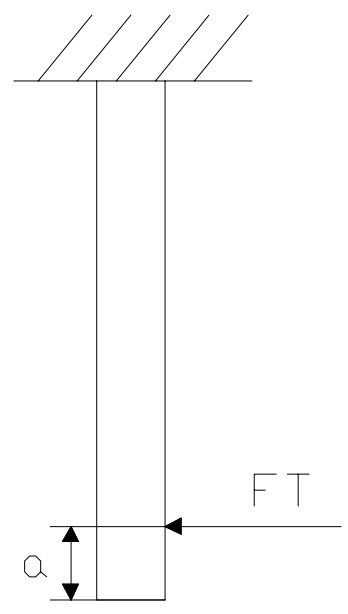

Figura 3.10. Fuerza concentrada

Con base en esta deformación de la herramienta se ha establecido un nuevo modelado de la deflexión. Donde $L$ es la longitud de la herramienta, $E$ su módulo de elasticidad e $I$ su momento de inercia y a la distancia del extremo de la viga al punto de aplicación de la fuerza. Esta expresión proporcionaría la deformación máxima de la herramienta, que tendrá lugar en el extremo inferior. Para cualquier otro punto de la herramienta, la expresión de la deformación será proporcional a la fuerza aplicada e inversamente proporcional al módulo de elasticidad y al momento de inercia. Si se considera el punto de aplicación de la fuerza, la deformación vendrá dada por la siguiente expresión: 


$$
d_{a}=\frac{F_{T}}{M}
$$

En la constante $M$ se engloban todos los parámetros incluidos en la expresión (3.58). Su valor se puede obtener experimentalmente calculando la constante de proporcionalidad entre el desplazamiento y la fuerza, según se explica en la sección siguiente. La deformación en un punto genérico será una función lineal de la distancia al extremo, por lo que puede expresarse de la siguiente manera:

$$
d(z)=A+B \cdot z
$$

La constante $B$ corresponderá a la inclinación de la herramienta que será igual a la derivada de la deformación respecto a la distancia al extremo empotrado. Como esta inclinación irá variando con la distancia se tomará el valor en el punto medio de la fuerza distribuida:

$$
B=y^{\prime}\left(L^{\prime}\right)
$$

Donde $L$ ' es la distancia del extremo de la herramienta al punto medio de la fuerza distribuida, según se verá en la sección siguiente.

Por otro lado, la deformación en el punto de aplicación de la fuerza es igual a la fuerza dividida por la constante $M$, según se indica en la expresión (3.59), por tanto:

$$
A+y^{\prime}\left(L^{\prime}\right) \cdot \frac{a_{p}}{2}=\frac{F_{T}}{M}
$$

De donde se puede deducir:

$$
A=\frac{F_{T}}{M}-y^{\prime}\left(L^{\prime}\right) \cdot \frac{a_{p}}{2}
$$

Sustituyendo esta expresión en la ecuación de la deformación (3.60) se obtiene: 


$$
d(z)=\frac{F_{T}}{M}-y^{\prime}\left(L^{\prime}\right) \cdot\left(\frac{a_{p}}{2}-z\right)(3.6
$$

La altura $z$ se puede expresar en términos de distancia angular teniendo en cuenta que:

$$
\frac{z}{a_{p}}=\frac{\alpha-\phi}{\delta}
$$

Donde $\delta$ es la distancia angular del filo implicada en el corte:

$$
\delta=\frac{2 \cdot a_{p} \cdot \tan \beta}{D}
$$

Combinando las expresiones (3.64) y (3.65) se obtiene:

$$
d(\alpha, \varphi)=\frac{F_{T}}{M}-y^{\prime}\left(L^{\prime}\right) \cdot a_{p} \cdot\left(\frac{1}{2}-\frac{\alpha-\phi}{\delta}\right)
$$

Sacando el factor común del cociente fuerza entre constante de rigidez se deduce la expresión general de la desviación de herramienta:

$$
d(\alpha, \phi)=\frac{F_{T}}{M} \cdot\left[1-y^{\prime}\left(L^{\prime}\right) \cdot \frac{M}{F_{T}} \cdot a_{p} \cdot\left(\frac{1}{2}-\frac{\alpha-\phi}{\delta}\right)\right]
$$

\subsubsection{Cálculo de constantes}

El valor que requiere el modelo, como se ha expuesto, es la derivada de la deformación con respecto a la distancia al extremo. Este valor se puede obtener partiendo del principio fundamental de la línea elástica en una barra sometida a tensión:

$$
y^{\prime \prime}(x)=\frac{M_{f}(x)}{E \cdot I}
$$


Por lo que:

$$
y^{\prime}(x)=\int_{0}^{x}-\frac{M_{f}(x)}{E \cdot I} \cdot d x
$$

El momento flector en cada punto se puede obtener a partir de la fuerza aplicada en la herramienta. El momento de inercia $I$ se puede calcular mediante las características geométricas de la herramienta, como se explica más adelante. En cuanto al módulo de elasticidad $E$, deberá obtenerse relacionando su valor con el de la constante $M$, medida experimentalmente, como también se explicará más adelante.

Habrá que obtener en primer lugar el momento flector en cada punto partiendo de la fuerza aplicada. En el esquema de la figura 3.11 se indica la fuerza que actúa sobre la herramienta y las reacciones en el empotramiento.

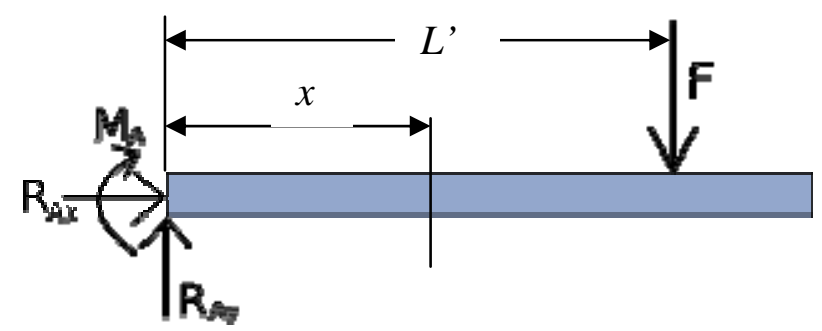

Figura 3.11. Fuerzas que actúan sobre la herramienta

Si se considera un punto situado a una distancia $x$ del extremo empotrado, el momento flector que se observa será:

$$
M_{f}(x)=M_{A}-R_{A y} \cdot x
$$

Planteando el equilibrio de fuerzas en la barra se obtiene:

$$
\begin{aligned}
& M_{A}=F \cdot L^{\prime} \\
& R_{A y}=F
\end{aligned}
$$


Por lo que:

$$
M_{f}(x)=F \cdot\left(L^{\prime}-x\right)(3.73)
$$

Para continuar el análisis es preciso introducir el momento de inercia de las distintas zonas de la herramienta. Dado que estos momentos de inercia dependen de la geometría se deberán distinguir los dos tipos de fresas utilizados en la investigación: Sandvik y DIXI.

\subsubsection{Fresas Sandvik}

El análisis de las distintas zonas de la herramienta se muestra en la figura 3.12. Se considera que la fuerza está aplicada en el último tramo de la fresa, de longitud $L_{5}$, que coincidirá, por tanto con el parámetro de corte $a_{p}$. El punto de aplicación estará a una distancia $L$ ' del empotramiento, que vendrá dada por:

$$
L^{\prime}=L_{1}+L_{2}+L_{3}+L_{4}+L_{5}-\frac{a_{p}}{2}
$$

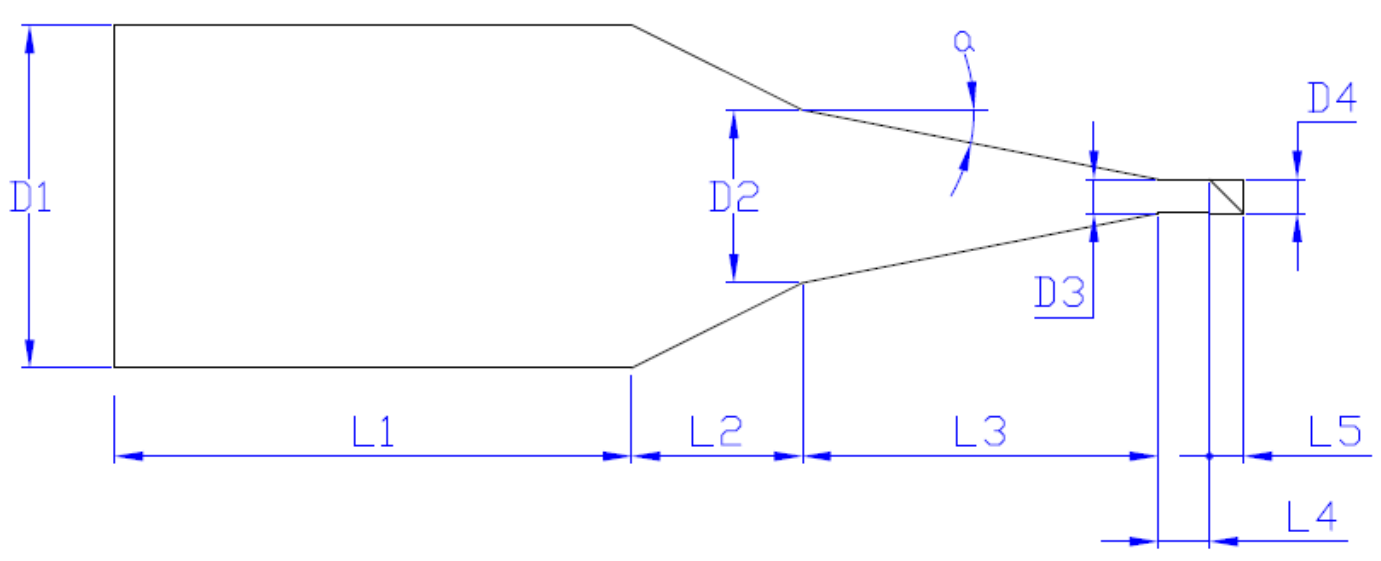

Figura 3.12. Croquis de una fresa Sandvik

Cada zona de la fresa tendrá su momento de inercia. Las zonas 1-4 tienen sección circular. Su momento de inercia será, por tanto: 


$$
I=\frac{\pi \cdot D^{4}}{64}
$$

La zona 5 tiene una geometría complicada, ya que consiste en un núcleo central con filos helicoidales a los lados. Esta sección va girando en torno al eje de la fresa a medida que se avanza por él. En la figura 3.13 se muestra una imagen del extremo de la herramienta.

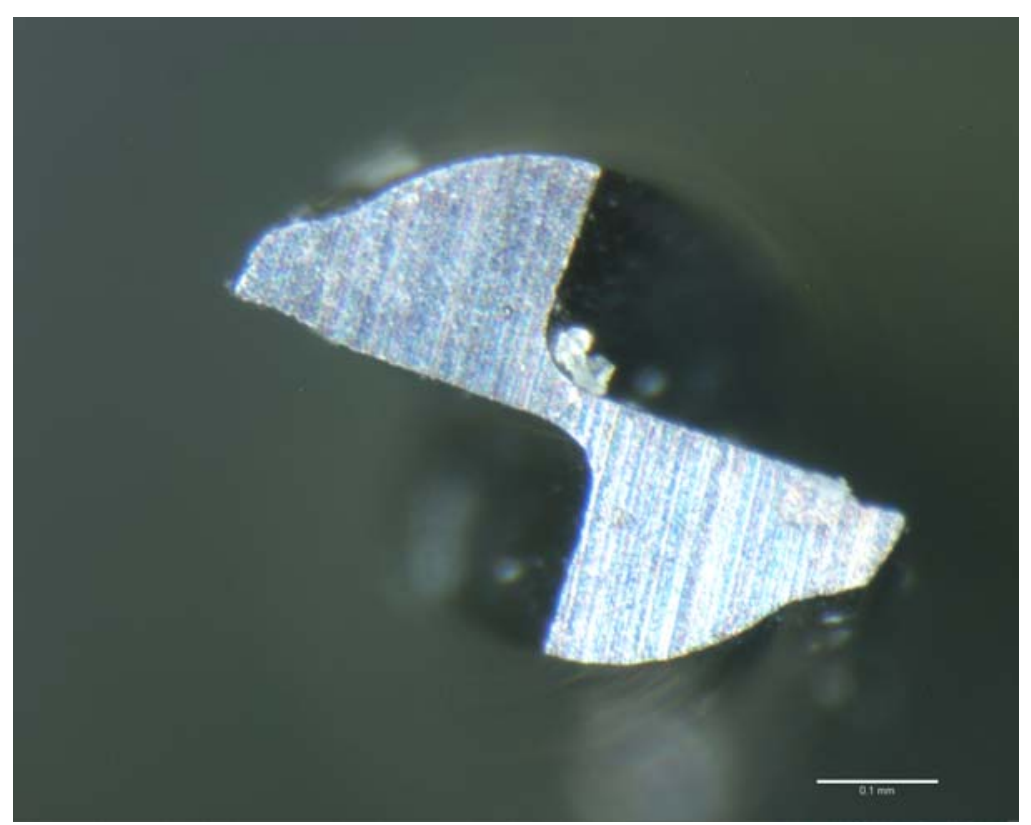

Figura 3.13. Imagen del extremo de una fresa Sandvik

El contorno de la fresa puede modelarse geométricamente según la figura 3.14. 


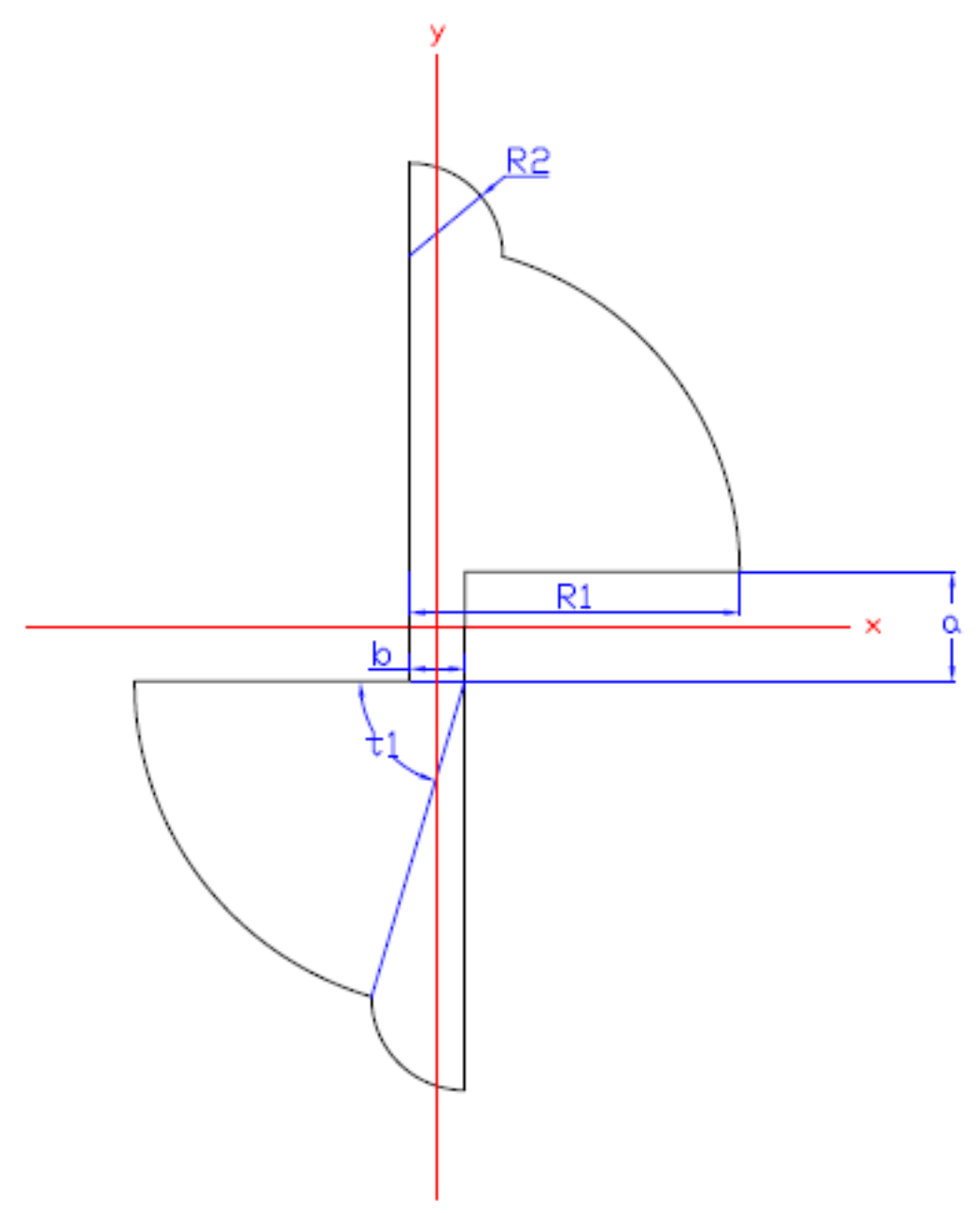

Figura 3.14. Croquis del extremo de una fresa Sandvik

El momento de inercia con respecto al plano que atraviesa la pieza por el eje se puede aproximar por el valor medio entre el momento con respecto al eje $x$ y con respecto al eje $y$ de la figura 3.13:

$$
I_{5}=\frac{I_{x}+I_{y}}{2}
$$

Utilizando los parámetros $a, b, R_{1}$ y $R_{2}$ según se indican en la figura 3.13 se obtiene la siguiente expresión: 


$$
\begin{aligned}
& I_{5}=\frac{a^{3} \cdot b}{12}+\frac{a^{2} \cdot R_{1}^{2}}{4} \cdot\left(\frac{\theta_{1}}{2}+\frac{\sin 2 \theta_{1}}{4}\right)+R_{1}^{4} \cdot\left(\frac{\theta_{1}}{2}+\frac{\sin 4 \theta_{1}}{16}\right)+ \\
& \frac{a \cdot R_{1}^{3}}{3} \cdot\left(1-\cos ^{3} \theta_{1}\right)+\frac{\pi}{4} \cdot R_{2}^{2} \cdot\left(\frac{D_{4}}{2}-R_{2}\right)^{2}+\frac{R_{2}^{3}}{3} \cdot\left(\frac{D_{4}}{2}-R_{2}\right)^{2}+ \\
& \frac{D_{4}-a-2 R_{2}}{6} \cdot\left[\left(R_{2}-\frac{b}{2}\right)^{3}+\left(\frac{b}{2}\right)^{3}\right]+\frac{3 \cdot \pi}{16} \cdot R_{2}{ }^{4}+\frac{\pi}{16} \cdot b^{2} \cdot R_{2}{ }^{2}- \\
& \frac{b \cdot R_{2}^{3}}{3}+\frac{\theta_{1} \cdot R_{1}^{4}}{8}+\frac{\sin 4 \theta_{1} \cdot R_{1}^{4}}{32}+\frac{b^{2} \cdot R_{1}^{2}}{16} \cdot\left(2 \theta_{1}-\sin 2 \theta_{1}\right)- \\
& \frac{b \cdot\left(R_{1} \cdot \sin 2 \theta_{1}\right)^{3}}{3}
\end{aligned}
$$

Una vez obtenidos los momentos de inercia se puede calcular la derivada de la elástica en cada punto mediante la expresión (3.70). En el punto de aplicación de la fuerza, la derivada será:

$$
y^{\prime}\left(L^{\prime}\right)=\frac{F}{E} \cdot\left[\begin{array}{l}
-\frac{32 \cdot L_{1}}{\pi \cdot D_{1}^{4}} \cdot\left(2 L^{\prime}-L_{1}\right)+\frac{64}{3 \cdot \pi} \cdot \frac{L_{2}}{D_{1}-D_{2}} \cdot \frac{L^{\prime}-L_{1}}{D_{1}^{3}} \\
+\frac{32}{3 \cdot \pi} \cdot\left(\frac{L_{2}}{D_{1}-D_{2}}\right)^{2} \cdot \frac{1}{D_{1}^{2}}-\frac{64}{3 \cdot \pi} \cdot \frac{L_{3}}{D_{2}-D_{3}} \cdot \frac{L^{\prime}-L_{1}-L_{2}-L_{3}}{D_{3}{ }^{3}}- \\
\frac{32}{3 \cdot \pi} \cdot\left(\frac{L_{3}}{D_{2}-D_{3}}\right)^{2} \cdot \frac{1}{D_{3}^{2}} \\
+\frac{32}{\pi \cdot D_{3}^{4}} \cdot\left[\left(L_{5}-\frac{a_{p}}{2}\right)^{2}-\left(L_{4}+L_{5}-\frac{a_{p}}{2}\right)^{2}\right]-\frac{1}{2 \cdot I_{5}} \cdot\left(L_{5}-\frac{a_{p}}{2}\right)^{2}
\end{array}\right]
$$

De manera análoga se puede obtener el valor de la deformación en cada punto a partir de la inclinación de la elástica mediante la relación siguiente:

$$
y(x)=\int_{0}^{x} y^{\prime}(x) \cdot d x
$$

A partir, por tanto, de la derivada de la elástica en cada tramo se obtiene la deformación en cada punto. En el punto de aplicación de la fuerza, la deformación será: 


$$
\begin{aligned}
& y\left(L^{\prime}\right)=-\frac{32 \cdot F \cdot L_{1}^{2}}{\pi \cdot E \cdot D_{1}{ }^{4}} \cdot\left(L^{\prime}-\frac{L_{1}}{3}\right)-\frac{32 \cdot F \cdot L_{1} \cdot L_{2}}{\pi \cdot E \cdot D_{1}^{4}} \cdot\left(2 L^{\prime}-L_{1}\right) \\
& +\frac{64 \cdot F \cdot L_{2}}{3 \cdot \pi \cdot E} \cdot \frac{L_{2}}{D_{1}-D_{2}} \cdot \frac{L^{\prime}-L_{1}}{D_{1}{ }^{3}}+\frac{32 \cdot F \cdot L_{2}}{3 \cdot \pi \cdot E} \cdot\left(\frac{L_{2}}{D_{1}-D_{2}}\right)^{2} \cdot \frac{1}{D_{1}{ }^{2}} \\
& -\frac{32 \cdot F}{3 \cdot \pi \cdot E} \cdot\left(\frac{L_{2}}{D_{1}-D_{2}}\right)^{2} \cdot\left(\frac{L^{\prime}-L_{1}-L_{2}}{D_{2}{ }^{2}}-\frac{L^{\prime}-L_{1}}{D_{1}{ }^{2}}\right) \\
& -\frac{64 \cdot F}{3 \cdot \pi \cdot E} \cdot\left(\frac{L_{2}}{D_{1}-D_{2}}\right)^{3} \cdot\left(\frac{1}{D_{2}}-\frac{1}{D_{1}}\right) \\
& -\frac{32 \cdot F \cdot L_{1} \cdot L_{3}}{\pi \cdot E \cdot D_{1}{ }^{4}} \cdot\left(2 L^{\prime}-L_{1}\right)-\frac{64 \cdot F \cdot L_{3}}{3 \cdot \pi \cdot E} \cdot \frac{L_{2}}{D_{1}-D_{2}} \cdot\left(\frac{L^{\prime}-L_{1}-L_{2}}{D_{2}{ }^{3}}-\frac{L^{\prime}-L_{1}}{D_{1}{ }^{3}}\right) \\
& -\frac{32 \cdot F \cdot L_{3}}{3 \cdot \pi \cdot E} \cdot\left(\frac{L_{2}}{D_{1}-D_{2}}\right)^{2} \cdot\left(\frac{1}{D_{2}^{2}}-\frac{1}{D_{1}^{2}}\right) \\
& +\frac{64 \cdot F \cdot L_{3}}{3 \cdot \pi \cdot E} \cdot \frac{L_{3}}{D_{2}-D_{3}} \cdot \frac{L^{\prime}-L_{1}-L_{2}}{D_{2}{ }^{3}}+\frac{32 \cdot F \cdot L_{3}}{3 \cdot \pi \cdot E} \cdot\left(\frac{L_{3}}{D_{2}-D_{3}}\right)^{2} \cdot \frac{1}{D_{2}{ }^{2}} \\
& -\frac{32 \cdot F}{3 \cdot \pi \cdot E} \cdot\left(\frac{L_{3}}{D_{2}-D_{3}}\right)^{2} \cdot\left(\frac{L^{\prime}-L_{1}-L_{2}-L_{3}}{D_{3}{ }^{2}}-\frac{L^{\prime}-L_{1}-L_{2}}{D_{2}{ }^{2}}\right) \\
& -\frac{64 \cdot F}{3 \cdot \pi \cdot E} \cdot\left(\frac{L_{3}}{D_{2}-D_{3}}\right)^{3} \cdot\left(\frac{1}{D_{3}}-\frac{1}{D_{2}}\right) \\
& -\frac{32 \cdot F \cdot L_{1} \cdot L_{4}}{\pi \cdot E \cdot D_{1}^{4}} \cdot\left(2 L^{\prime}-L_{1}\right)+\frac{64 \cdot F \cdot L_{4}}{3 \cdot \pi \cdot E} \cdot \frac{L_{2}}{D_{1}-D_{2}} \cdot \frac{L^{\prime}-L_{1}}{D_{1}^{3}} \\
& +\frac{32 \cdot F \cdot L_{4}}{3 \cdot \pi \cdot E} \cdot\left(\frac{L_{2}}{D_{1}-D_{2}}\right)^{2} \cdot \frac{1}{D_{1}{ }^{2}}-\frac{64 \cdot F \cdot L_{4}}{3 \cdot \pi \cdot E} \cdot \frac{L_{3}}{D_{2}-D_{3}} \cdot \frac{L^{\prime}-L_{1}-L_{2}-L_{3}}{D_{3}{ }^{3}} \\
& -\frac{32 \cdot F \cdot L_{4}}{3 \cdot \pi \cdot E} \cdot\left(\frac{L_{3}}{D_{2}-D_{3}}\right)^{2} \cdot \frac{1}{D_{3}{ }^{2}}-\frac{32 \cdot F}{3 \cdot \pi \cdot E \cdot D_{3}{ }^{4}} \cdot\left[\left(L_{5}-\frac{a_{p}}{2}\right)^{3}-\left(L_{4}+L_{5}-\frac{a_{p}}{2}\right)^{3}\right] \\
& -\frac{32 \cdot F \cdot L_{4}}{\pi \cdot E \cdot D_{3}^{4}}\left(L_{4}+L_{5}-\frac{a_{p}}{2}\right)-\frac{32 \cdot F \cdot L_{1}}{\pi \cdot E \cdot D_{1}{ }^{4}} \cdot\left(2 L^{\prime}-L_{1}\right) \cdot\left(L_{5}-\frac{a_{p}}{2}\right) \\
& +\frac{64 \cdot F}{3 \cdot \pi \cdot E} \cdot \frac{L_{2}}{D_{1}-D_{2}} \cdot \frac{L^{\prime}-L_{1}}{D_{1}^{3}} \cdot\left(L_{5}-\frac{a_{p}}{2}\right)+\frac{32 \cdot F}{3 \cdot \pi \cdot E} \cdot\left(\frac{L_{2}}{D_{1}-D_{2}}\right)^{2} \cdot \frac{1}{D_{1}^{2}} \cdot\left(L_{5}-\frac{a_{p}}{2}\right) \\
& -\frac{64 \cdot F}{3 \cdot \pi \cdot E} \cdot \frac{L_{3}}{D_{2}-D_{3}} \cdot \frac{L^{\prime}-L_{1}-L_{2}-L_{3}}{D_{3}{ }^{3}} \cdot\left(L_{5}-\frac{a_{p}}{2}\right) \\
& -\frac{32 \cdot F}{3 \cdot \pi \cdot E} \cdot\left(\frac{L_{3}}{D_{2}-D_{3}}\right)^{2} \cdot \frac{1}{D_{3}^{2}} \cdot\left(L_{5}-\frac{a_{p}}{2}\right) \\
& +\frac{32 \cdot F}{\pi \cdot E \cdot D_{3}{ }^{4}} \cdot\left[\left(L_{5}-\frac{a_{p}}{2}\right)^{2}-\left(L_{4}+L_{5}-\frac{a_{p}}{2}\right)^{2}\right] \cdot\left(L_{5}-\frac{a_{p}}{2}\right)-\frac{F}{3 \cdot E \cdot I_{5}} \cdot\left(L_{5}-\frac{a_{p}}{2}\right)^{3}
\end{aligned}
$$


Pasando al primer miembro los valores de la fuerza y el módulo de elasticidad, se obtiene: 


$$
\begin{aligned}
& y\left(L^{\prime}\right) \cdot \frac{E}{F}=-\frac{32 \cdot L_{1}{ }^{2}}{\pi \cdot D_{1}{ }^{4}} \cdot\left(L^{\prime}-\frac{L_{1}}{3}\right)-\frac{32 \cdot L_{1} \cdot L_{2}}{\pi \cdot D_{1}{ }^{4}} \cdot\left(2 L^{\prime}-L_{1}\right) \\
& +\frac{64 \cdot L_{2}}{3 \cdot \pi} \cdot \frac{L_{2}}{D_{1}-D_{2}} \cdot \frac{L^{\prime}-L_{1}}{D_{1}^{3}}+\frac{32 \cdot L_{2}}{3 \cdot \pi} \cdot\left(\frac{L_{2}}{D_{1}-D_{2}}\right)^{2} \cdot \frac{1}{D_{1}^{2}} \\
& -\frac{32}{3 \cdot \pi} \cdot\left(\frac{L_{2}}{D_{1}-D_{2}}\right)^{2} \cdot\left(\frac{L^{\prime}-L_{1}-L_{2}}{D_{2}{ }^{2}}-\frac{L^{\prime}-L_{1}}{D_{1}^{2}}\right)-\frac{64}{3 \cdot \pi} \cdot\left(\frac{L_{2}}{D_{1}-D_{2}}\right)^{3} \cdot\left(\frac{1}{D_{2}}-\frac{1}{D_{1}}\right) \\
& -\frac{32 \cdot L_{1} \cdot L_{3}}{\pi \cdot D_{1}{ }^{4}} \cdot\left(2 L^{\prime}-L_{1}\right)-\frac{64 \cdot L_{3}}{3 \cdot \pi} \cdot \frac{L_{2}}{D_{1}-D_{2}} \cdot\left(\frac{L^{\prime}-L_{1}-L_{2}}{D_{2}{ }^{3}}-\frac{L^{\prime}-L_{1}}{D_{1}^{3}}\right) \\
& -\frac{32 \cdot L_{3}}{3 \cdot \pi} \cdot\left(\frac{L_{2}}{D_{1}-D_{2}}\right)^{2} \cdot\left(\frac{1}{D_{2}^{2}}-\frac{1}{D_{1}^{2}}\right) \\
& +\frac{64 \cdot L_{3}}{3 \cdot \pi} \cdot \frac{L_{3}}{D_{2}-D_{3}} \cdot \frac{L^{\prime}-L_{1}-L_{2}}{D_{2}{ }^{3}}+\frac{32 \cdot L_{3}}{3 \cdot \pi} \cdot\left(\frac{L_{3}}{D_{2}-D_{3}}\right)^{2} \cdot \frac{1}{D_{2}{ }^{2}} \\
& -\frac{32}{3 \cdot \pi} \cdot\left(\frac{L_{3}}{D_{2}-D_{3}}\right)^{2} \cdot\left(\frac{L^{\prime}-L_{1}-L_{2}-L_{3}}{D_{3}{ }^{2}}-\frac{L^{\prime}-L_{1}-L_{2}}{D_{2}{ }^{2}}\right) \\
& -\frac{64}{3 \cdot \pi} \cdot\left(\frac{L_{3}}{D_{2}-D_{3}}\right)^{3} \cdot\left(\frac{1}{D_{3}}-\frac{1}{D_{2}}\right)-\frac{32 \cdot L_{1} \cdot L_{4}}{\pi \cdot D_{1}^{4}} \cdot\left(2 L^{\prime}-L_{1}\right) \\
& +\frac{64 \cdot L_{4}}{3 \cdot \pi} \cdot \frac{L_{2}}{D_{1}-D_{2}} \cdot \frac{L^{\prime}-L_{1}}{D_{1}^{3}}+\frac{32 \cdot L_{4}}{3 \cdot \pi} \cdot\left(\frac{L_{2}}{D_{1}-D_{2}}\right)^{2} \cdot \frac{1}{D_{1}^{2}} \\
& -\frac{64 \cdot L_{4}}{3 \cdot \pi} \cdot \frac{L_{3}}{D_{2}-D_{3}} \cdot \frac{L^{\prime}-L_{1}-L_{2}-L_{3}}{D_{3}^{3}} \\
& -\frac{32 \cdot L_{4}}{3 \cdot \pi} \cdot\left(\frac{L_{3}}{D_{2}-D_{3}}\right)^{2} \cdot \frac{1}{D_{3}^{2}}-\frac{32}{3 \cdot \pi \cdot D_{3}^{4}} \cdot\left[\left(L_{5}-\frac{a_{p}}{2}\right)^{3}-\left(L_{4}+L_{5}-\frac{a_{p}}{2}\right)^{3}\right] \\
& -\frac{32 \cdot L_{4}}{\pi \cdot D_{3}{ }^{4}} \cdot\left(L_{4}+L_{5}-\frac{a_{p}}{2}\right)^{2}-\frac{32 \cdot L_{1}}{\pi \cdot D_{1}^{4}} \cdot\left(2 L^{\prime}-L_{1}\right) \cdot\left(L_{5}-\frac{a_{p}}{2}\right) \\
& +\frac{64}{3 \cdot \pi} \cdot \frac{L_{2}}{D_{1}-D_{2}} \cdot \frac{L^{\prime}-L_{1}}{D_{1}^{3}} \cdot\left(L_{5}-\frac{a_{p}}{2}\right)+\frac{32}{3 \cdot \pi} \cdot\left(\frac{L_{2}}{D_{1}-D_{2}}\right)^{2} \cdot \frac{1}{D_{1}^{2}} \cdot\left(L_{5}-\frac{a_{p}}{2}\right) \\
& -\frac{64}{3 \cdot \pi} \cdot \frac{L_{3}}{D_{2}-D_{3}} \cdot \frac{L^{\prime}-L_{1}-L_{2}-L_{3}}{D_{3}^{3}} \cdot\left(L_{5}-\frac{a_{p}}{2}\right) \\
& -\frac{32}{3 \cdot \pi} \cdot\left(\frac{L_{3}}{D_{2}-D_{3}}\right)^{2} \cdot \frac{1}{D_{3}^{2}} \cdot\left(L_{5}-\frac{a_{p}}{2}\right) \\
& +\frac{32}{\pi \cdot D_{3}{ }^{4}} \cdot\left[\left(L_{5}-\frac{a_{p}}{2}\right)^{2}-\left(L_{4}+L_{5}-\frac{a_{p}}{2}\right)^{2}\right] \cdot\left(L_{5}-\frac{a_{p}}{2}\right)-\frac{1}{3 \cdot I_{5}} \cdot\left(L_{5}-\frac{a_{p}}{2}\right)^{3}
\end{aligned}
$$


Por la referencia que se ha tomado en la figura 3.11 el valor de la deformada será negativo, por lo que para relacionar la deformada con la desviación de la expresión (3.59) habrá que cambiar su signo:

$$
d_{a}=-y\left(L^{\prime}\right)=\frac{F_{T}}{M}
$$

Por lo que:

$$
\frac{y\left(L^{\prime}\right)}{F_{T}}=-\frac{1}{M}
$$

Identificando la fuerza $F$ con la fuerza total de la expresión (3.83) se obtiene el módulo de elasticidad:

$$
E=y\left(L^{\prime}\right) \cdot \frac{E}{F} \cdot \frac{F}{y\left(L^{\prime}\right)}=-M \cdot y\left(L^{\prime}\right) \cdot \frac{E}{F}
$$

Sustituyendo la expresión (3.81) se obtiene: 


$$
\begin{aligned}
& {\left[-\frac{32 \cdot L_{1}^{2}}{\pi \cdot D_{1}^{4}} \cdot\left(L^{\prime}-\frac{L_{1}}{3}\right)-\frac{32 \cdot L_{1} \cdot L_{2}}{\pi \cdot D_{1}^{4}} \cdot\left(2 L^{\prime}-L_{1}\right)\right.} \\
& +\frac{64 \cdot L_{2}}{3 \cdot \pi} \cdot \frac{L_{2}}{D_{1}-D_{2}} \cdot \frac{L^{\prime}-L_{1}}{D_{1}^{3}}+\frac{32 \cdot L_{2}}{3 \cdot \pi} \cdot\left(\frac{L_{2}}{D_{1}-D_{2}}\right)^{2} \cdot \frac{1}{D_{1}^{2}} \\
& -\frac{32}{3 \cdot \pi} \cdot\left(\frac{L_{2}}{D_{1}-D_{2}}\right)^{2} \cdot\left(\frac{L^{\prime}-L_{1}-L_{2}}{D_{2}^{2}}-\frac{L^{\prime}-L_{1}}{D_{1}^{2}}\right)-\frac{64}{3 \cdot \pi} \cdot\left(\frac{L_{2}}{D_{1}-D_{2}}\right)^{3} \cdot\left(\frac{1}{D_{2}}-\frac{1}{D_{1}}\right) \\
& -\frac{32 \cdot L_{1} \cdot L_{3}}{\pi \cdot D_{1}^{4}} \cdot\left(2 L^{\prime}-L_{1}\right)-\frac{64 \cdot L_{3}}{3 \cdot \pi} \cdot \frac{L_{2}}{D_{1}-D_{2}} \cdot\left(\frac{L^{\prime}-L_{1}-L_{2}}{D_{2}{ }^{3}}-\frac{L^{\prime}-L_{1}}{D_{1}^{3}}\right) \\
& -\frac{32 \cdot L_{3}}{3 \cdot \pi} \cdot\left(\frac{L_{2}}{D_{1}-D_{2}}\right)^{2} \cdot\left(\frac{1}{D_{2}^{2}}-\frac{1}{D_{1}^{2}}\right) \\
& +\frac{64 \cdot L_{3}}{3 \cdot \pi} \cdot \frac{L_{3}}{D_{2}-D_{3}} \cdot \frac{L^{\prime}-L_{1}-L_{2}}{D_{2}{ }^{2}}+\frac{32 \cdot L_{3}}{3 \cdot \pi} \cdot\left(\frac{L_{3}}{D_{2}-D_{3}}\right)^{2} \cdot \frac{1}{D_{2}^{2}} \\
& -\frac{32}{3 \cdot \pi} \cdot\left(\frac{L_{3}}{D_{2}-D_{3}}\right)^{2} \cdot\left(\frac{L^{\prime}-L_{1}-L_{2}-L_{3}}{D_{3}^{2}}-\frac{L^{\prime}-L_{1}-L_{2}}{D_{2}^{2}}\right) \\
& E=-M \cdot-\frac{64}{3 \cdot \pi} \cdot\left(\frac{L_{3}}{D_{2}-D_{3}}\right)^{3} \cdot\left(\frac{1}{D_{3}}-\frac{1}{D_{2}}\right)-\frac{32 \cdot L_{1} \cdot L_{4}}{\pi \cdot D_{1}^{4}} \cdot\left(2 L^{\prime}-L_{1}\right) \\
& +\frac{64 \cdot L_{4}}{3 \cdot \pi} \cdot \frac{L_{2}}{D_{1}-D_{2}} \cdot \frac{L^{\prime}-L_{1}}{D_{1}^{3}}+\frac{32 \cdot L_{4}}{3 \cdot \pi} \cdot\left(\frac{L_{2}}{D_{1}-D_{2}}\right)^{2} \cdot \frac{1}{D_{1}^{2}} \\
& -\frac{64 \cdot L_{4}}{3 \cdot \pi} \cdot \frac{L_{3}}{D_{2}-D_{3}} \cdot \frac{L^{\prime}-L_{1}-L_{2}-L_{3}}{D_{3}^{3}} \\
& -\frac{32 \cdot L_{4}}{3 \cdot \pi} \cdot\left(\frac{L_{3}}{D_{2}-D_{3}}\right)^{2} \cdot \frac{1}{D_{3}{ }^{2}}-\frac{32}{3 \cdot \pi \cdot D_{3}{ }^{4}} \cdot\left[\left(L_{5}-\frac{a_{p}}{2}\right)^{3}-\left(L_{4}+L_{5}-\frac{a_{p}}{2}\right)^{3}\right] \\
& -\frac{32 \cdot L_{4}}{\pi \cdot D_{3}^{4}} \cdot\left(L_{4}+L_{5}-\frac{a_{p}}{2}\right)^{2}-\frac{32 \cdot L_{1}}{\pi \cdot D_{1}^{4}} \cdot\left(2 L^{\prime}-L_{1}\right) \cdot\left(L_{5}-\frac{a_{p}}{2}\right) \\
& +\frac{64}{3 \cdot \pi} \cdot \frac{L_{2}}{D_{1}-D_{2}} \cdot \frac{L^{\prime}-L_{1}}{D_{1}^{3}} \cdot\left(L_{5}-\frac{a_{p}}{2}\right)+\frac{32}{3 \cdot \pi} \cdot\left(\frac{L_{2}}{D_{1}-D_{2}}\right)^{2} \cdot \frac{1}{D_{1}^{2}} \cdot\left(L_{5}-\frac{a_{p}}{2}\right) \\
& -\frac{64}{3 \cdot \pi} \cdot \frac{L_{3}}{D_{2}-D_{3}} \cdot \frac{L^{\prime}-L_{1}-L_{2}-L_{3}}{D_{3}{ }^{3}} \cdot\left(L_{5}-\frac{a_{p}}{2}\right) \\
& -\frac{32}{3 \cdot \pi} \cdot\left(\frac{L_{3}}{D_{2}-D_{3}}\right)^{2} \cdot \frac{1}{D_{3}^{2}} \cdot\left(L_{5}-\frac{a_{p}}{2}\right) \\
& +\frac{32}{\pi \cdot D_{3}^{4}} \cdot\left[\left(L_{5}-\frac{a_{p}}{2}\right)^{2}-\left(L_{4}+L_{5}-\frac{a_{p}}{2}\right)^{2}\right] \cdot\left(L_{5}-\frac{a_{p}}{2}\right)-\frac{1}{3 \cdot I_{5}} \cdot\left(L_{5}-\frac{a_{p}}{2}\right)^{3}
\end{aligned}
$$


La constante $M$ se determina experimentalmente, como se explica en el capítulo 7.

El término que acompaña a la constante $M$ se denomina $K_{1}$ y es una constante que depende únicamente de las características geométricas de la herramienta. De esa manera, la expresión anterior se reduce a:

$$
E=-M \cdot K_{1}(3.86)
$$

El módulo de elasticidad así obtenido se puede sustituir en la expresión (3.78) de la derivada de la elástica:

$$
y^{\prime}\left(L^{\prime}\right)=-\frac{F}{M \cdot K_{1}} \cdot\left[\begin{array}{l}
-\frac{32 \cdot L_{1}}{\pi \cdot D_{1}^{4}} \cdot\left(2 L^{\prime}-L_{1}\right)+\frac{64}{3 \cdot \pi} \cdot \frac{L_{2}}{D_{1}-D_{2}} \cdot \frac{L^{\prime}-L_{1}}{D_{1}{ }^{3}} \\
+\frac{32}{3 \cdot \pi} \cdot\left(\frac{L_{2}}{D_{1}-D_{2}}\right)^{2} \cdot \frac{1}{D_{1}^{2}}-\frac{64}{3 \cdot \pi} \cdot \frac{L_{3}}{D_{2}-D_{3}} \cdot \frac{L^{\prime}-L_{1}-L_{2}-L_{3}}{D_{3}{ }^{3}}-\left(\frac{L_{3}}{D_{2}-D_{3}}\right)^{2} \cdot \frac{1}{D_{3}^{2}} \\
+\frac{32}{\pi \cdot D_{3}{ }^{4}} \cdot\left[\left(L_{5}-\frac{a_{p}}{2}\right)^{2}-\left(L_{4}+L_{5}-\frac{a_{p}}{2}\right)^{2}\right]
\end{array}\right]
$$

Igual que se hizo anteriormente, la expresión entre corchetes de esta ecuación se denomina $K_{2}$. De esta manera:

$$
y^{\prime}\left(L^{\prime}\right)=-\frac{F}{M} \cdot \frac{K_{2}}{K_{1}}
$$

Identificando el valor de la fuerza $F$ con la fuerza total de la expresión (3.68) y sustituyendo este valor de la derivada de la elástica se obtiene:

$$
d(\alpha, \phi)=\frac{F_{T}}{M} \cdot\left[1-\frac{K_{2}}{K_{1}} \cdot a_{p} \cdot\left(\frac{1}{2}-\frac{\alpha-\phi}{\delta}\right)\right]
$$


Que proporcionaría el valor de la desviación del filo debido a la deflexión de la herramienta en cada posición del filo expresada con el valor $\alpha$ y para cada altura considerada expresada mediante la variable $\phi$.

\subsubsection{Fresas DIXI}

Las fresas DIXI tienen una geometría más sencilla que las Sandvik. En la figura 3.15 se muestra el croquis lateral de la herramienta con sus medidas, en el que se ve que tiene únicamente 3 zonas. Sobre el croquis se ha señalado también la posición del punto de aplicación de la fuerza de corte, cuya distancia al extremo de la fresa es la mitad de la profundidad radial de corte.

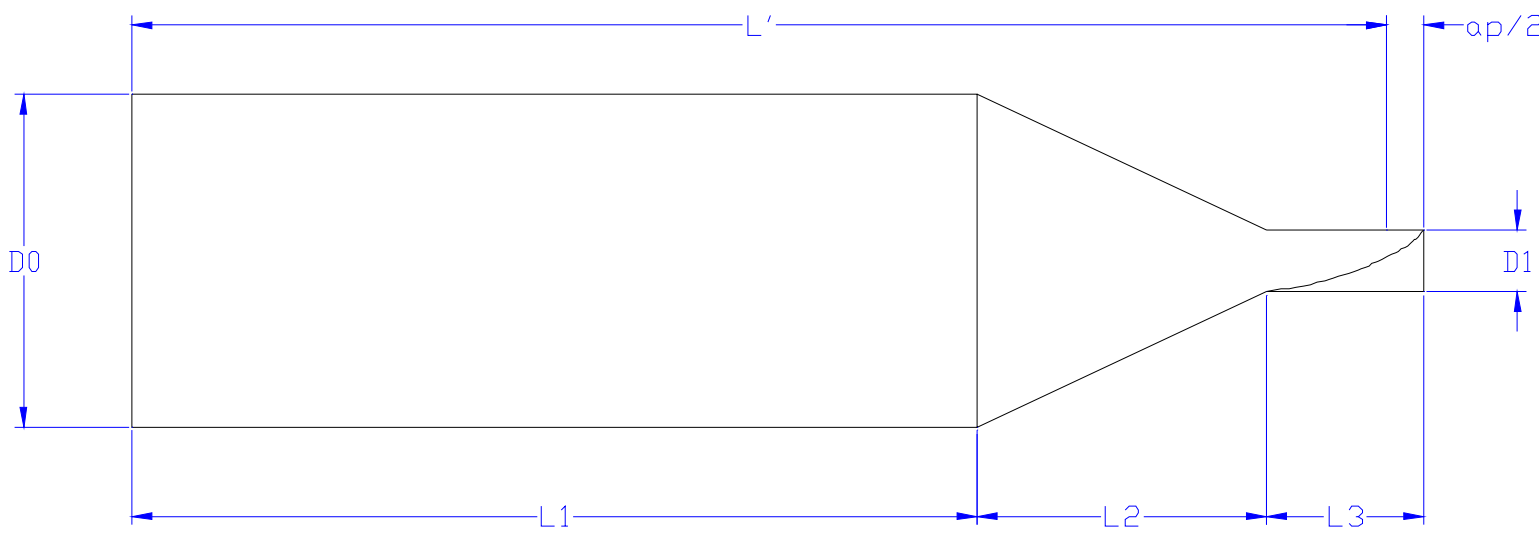

Figura 3.15. Croquis de una fresa DIXI

Como se hizo con las fresas Sandvik se ha de caracterizar la geometría del extremo de la herramienta para poder calcular el momento de inercia del último tramo. En la figura 3.16 se muestran los contornos del extremo de una fresa DIXI de $0.6 \mathrm{~mm}$ de diámetro, que se modela geométricamente según el dibujo de la figura 3.17. 


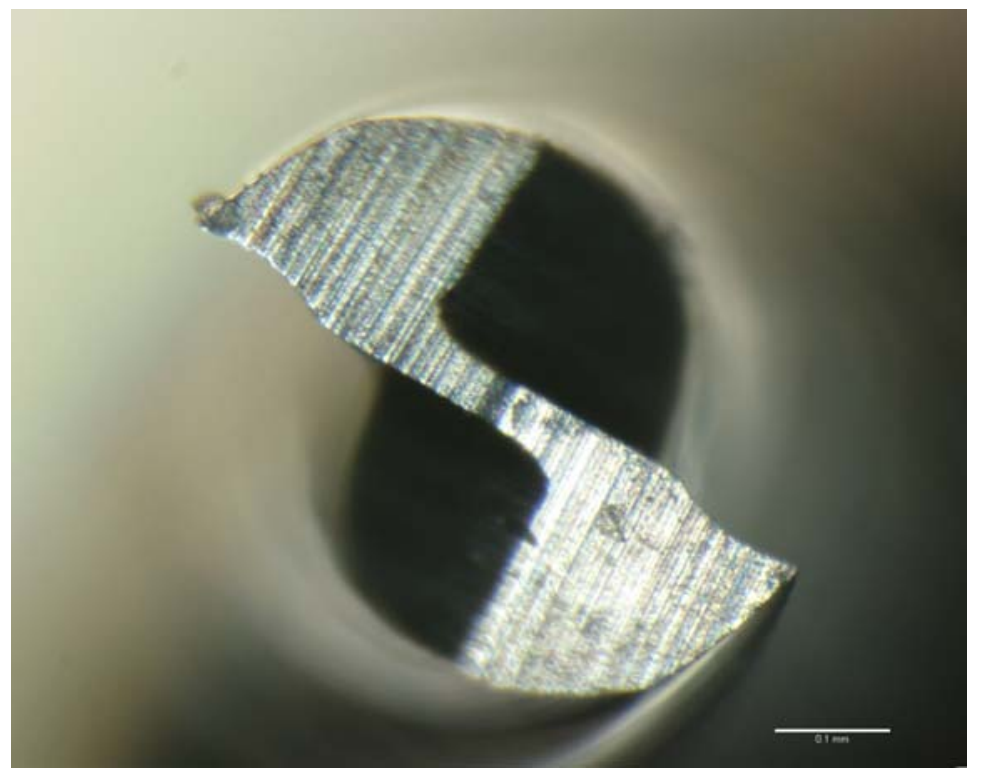

Figura 3.16. Imagen del extremo de una fresa DIXI 


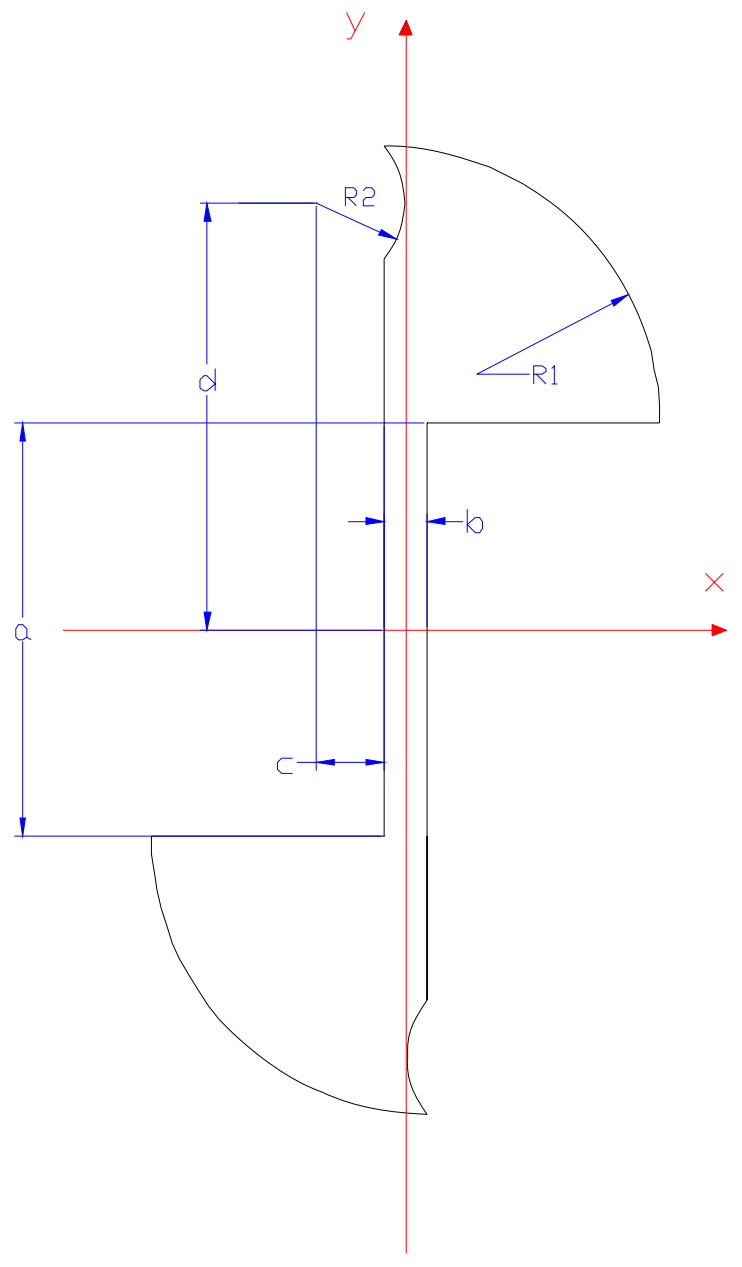

Figura 3.17. Croquis del extremo de una fresa DIXI

Para calcular el momento de inercia del último tramo de la fresa se utilizará la expresión vista en el apartado anterior:

$$
I_{3}=\frac{I_{x}+I_{y}}{2}(3.90)
$$

Realizando las operaciones requeridas se obtiene la siguiente expresión: 


$$
\begin{aligned}
& I_{3}=\frac{9 \cdot a^{3} \cdot b}{24}+\frac{\pi \cdot a^{2} \cdot R_{1}^{2}}{16}+\frac{3 \cdot \pi \cdot R_{1}^{4}}{16}+\frac{(a-b) \cdot R_{1}^{3}}{3}+\frac{\pi \cdot b^{2} \cdot R_{1}^{2}}{16} \\
& +\frac{d^{2} \cdot R_{1} \cdot R_{2}}{2} \cdot\left(\theta_{2}-\theta_{1}\right)-\frac{d^{2} \cdot R_{1} \cdot R_{2}}{4} \cdot\left(\sin 2 \theta_{2}-\sin 2 \theta_{1}\right) \\
& +c \cdot d^{2} \cdot R_{1} \cdot\left(\cos \theta_{2}-\cos \theta_{1}\right)+\frac{R_{1} \cdot R_{2}^{3}}{8} \cdot\left(\theta_{2}-\theta_{1}\right) \\
& -\frac{R_{1} \cdot R_{2}{ }^{3}}{32} \cdot\left(\sin 4 \theta_{2}-\sin 4 \theta_{1}\right)+\frac{c \cdot R_{1} \cdot R_{2}{ }^{2}}{3} \cdot\left(\cos ^{3} \theta_{2}-\cos ^{3} \theta_{1}\right) \\
& -\frac{2 \cdot d \cdot R_{1} \cdot R_{2}{ }^{2}}{3} \cdot\left(\sin ^{3} \theta_{2}-\sin ^{3} \theta_{1}\right)-\frac{c \cdot d \cdot R_{1} \cdot R_{2}}{2} \cdot\left(\cos 2 \theta_{2}-\cos 2 \theta_{1}\right) \\
& -R_{2}{ }^{2} \cdot\left(c+\frac{b}{2}\right)^{2} \cdot \theta_{3}+\frac{R_{2}{ }^{2}}{2} \cdot\left(c+\frac{b}{2}\right)^{2} \cdot \sin 2 \theta_{3}-\frac{R_{2}{ }^{4}}{4} \cdot \theta_{3}+\frac{R_{2}{ }^{4}}{16} \cdot \sin 4 \theta_{3} \\
& +\frac{2 \cdot R_{2}{ }^{3} \cdot(b+2 c)}{3} \cdot \sin ^{3} \theta_{3}
\end{aligned}
$$

Donde:

$$
\begin{aligned}
& \theta_{1}=\arcsin \frac{c}{R_{2}} \\
& \theta_{2}=\arccos \frac{\frac{a}{2}-d+R_{1}}{R_{2}} \\
& \theta_{3}=\frac{\pi}{2}-\arcsin \frac{c}{R_{2}}
\end{aligned}
$$

Siguiendo el mismo procedimiento del apartado anterior se pueden determinar las constantes $K_{1}$ y $K_{2}$ para obtener la deflexión de herramienta: 


$$
\begin{aligned}
& K_{1}=-\frac{32 \cdot L_{1}{ }^{2}}{\pi \cdot D_{0}{ }^{4}} \cdot\left(L^{\prime}-\frac{L_{1}}{3}\right)-\frac{32 \cdot L_{1} \cdot L_{2}}{\pi \cdot D_{0}{ }^{2}} \cdot\left(2 L^{\prime}-L_{1}\right) \\
& +\frac{64 \cdot L_{2}{ }^{2}}{3 \cdot \pi \cdot\left(D_{0}-D_{1}\right)} \cdot\left[\frac{L^{\prime}-L_{1}}{D_{0}{ }^{3}}+\frac{L_{2}}{2 \cdot D_{0}{ }^{2} \cdot\left(D_{0}-D_{1}\right)}\right] \\
& -\frac{32 \cdot L_{2}{ }^{2}}{3 \cdot \pi \cdot\left(D_{0}-D_{1}\right)^{2}} \cdot\left(\frac{L^{\prime}-L_{1}-L_{2}}{D_{1}{ }^{2}}-\frac{L^{\prime}-L_{1}}{D_{0}{ }^{2}}+\frac{1}{D_{1}}-\frac{1}{D_{0}}\right) \\
& -\frac{16 \cdot L_{2}{ }^{3}}{3 \cdot \pi \cdot\left(D_{0}-D_{1}\right)^{3}} \cdot\left(\frac{1}{D_{1}}-\frac{1}{D_{0}}\right) \\
& -\frac{32 \cdot L_{1}}{\pi \cdot D_{0}{ }^{4}} \cdot\left(2 L^{\prime}-L_{1}\right) \cdot\left(L^{\prime}-L_{1}-L_{2}\right) \\
& -\frac{64 \cdot L_{2} \cdot\left(L^{\prime}-L_{1}-L_{2}\right)}{3 \cdot \pi \cdot\left(D_{0}-D_{1}\right)} \cdot\left[\frac{L^{\prime}-L_{1}-L_{2}}{D_{1}{ }^{3}}-\frac{L^{\prime}-L_{1}}{D_{0}{ }^{3}}+\frac{L_{2}}{2 \cdot\left(D_{0}-D_{1}\right)} \cdot\left(\frac{1}{D_{1}{ }^{2}}-\frac{1}{D_{0}{ }^{2}}\right)\right] \\
& -\frac{1}{3 \cdot I_{3}} \cdot\left(L^{\prime}-L_{1}-L_{2}\right)^{3} \\
& \quad K_{2}=-\frac{32 \cdot L_{1}}{\pi \cdot D_{0}{ }^{4}} \cdot\left(2 L^{\prime}-L_{1}\right) \\
& \quad-\frac{64 \cdot L_{2}}{3 \cdot \pi \cdot\left(D_{0}-D_{1}\right)} \cdot\left[\frac{L^{\prime}-L_{1}-L_{2}}{D_{1}{ }^{3}}-\frac{L^{\prime}-L_{1}}{D_{0}{ }^{3}}+\frac{L_{2}}{2 \cdot\left(D_{0}-D_{1}\right)} \cdot\left(\frac{1}{D_{1}{ }^{2}}-\frac{1}{D_{0}{ }^{2}}\right)\right] \\
& \quad-\frac{1}{2 \cdot I_{3}} \cdot\left(L^{\prime}-L_{1}-L_{2}\right)^{2}
\end{aligned}
$$




\section{DESARROLLO DEL MODELO}

\subsection{Microfresado en oposición}

4.1.1 Procedimiento de cálculo del espesor de viruta en condiciones de corte simétrico

Para obtener la expresión del nuevo espesor de viruta se puede utilizar el esquema de la figura 4.1:

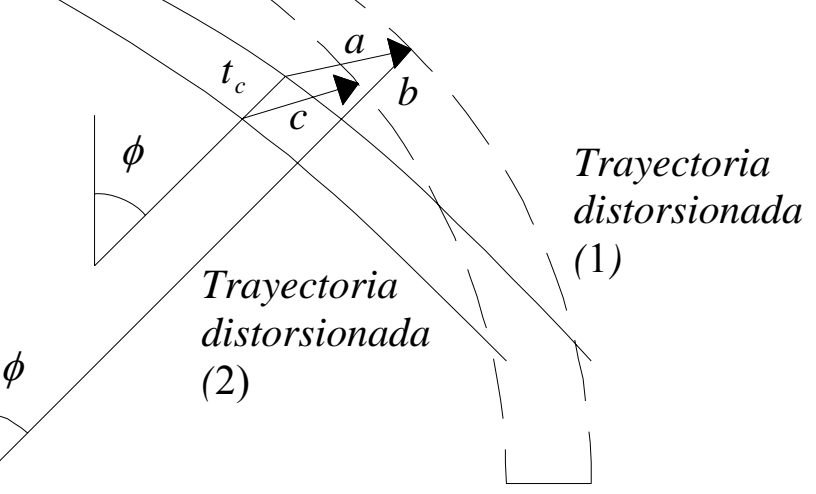

Figura 4.1. Trayectorias de la herramienta distorsionadas por la deflexión de herramienta

Las distancias entre las trayectorias ideales y las reales se pueden expresar de la siguiente manera:

$$
\begin{gathered}
a=\left[1-\frac{K_{2}}{K_{1}} \cdot a_{p} \cdot\left(\frac{1}{2}-\frac{\alpha-\phi}{\delta}\right)\right] \cdot \frac{F_{T}(1)}{M} \\
c=\left[1-\frac{K_{2}}{K_{1}} \cdot a_{p} \cdot\left(\frac{1}{2}-\frac{\alpha-\phi}{\delta}\right)\right] \cdot \frac{F_{T}(2)}{M}
\end{gathered}
$$


La distancia $\mathrm{b}$ entre las trayectorias de los dos dientes no distorsionadas por la deflexión vendrá dada por la expresión (42):

$$
b=t_{c 0}=f_{z} \cdot \sin \phi+2 \cdot \rho \cdot \cos (\Delta \phi-\lambda)
$$

$\phi$ es el ángulo de posición de la herramienta sin distorsión, que se considera igual al de la herramienta con distorsión.

En la figura 4.2 se muestra un esquema más detallado del espesor de viruta en condiciones de deflexión de herramienta.

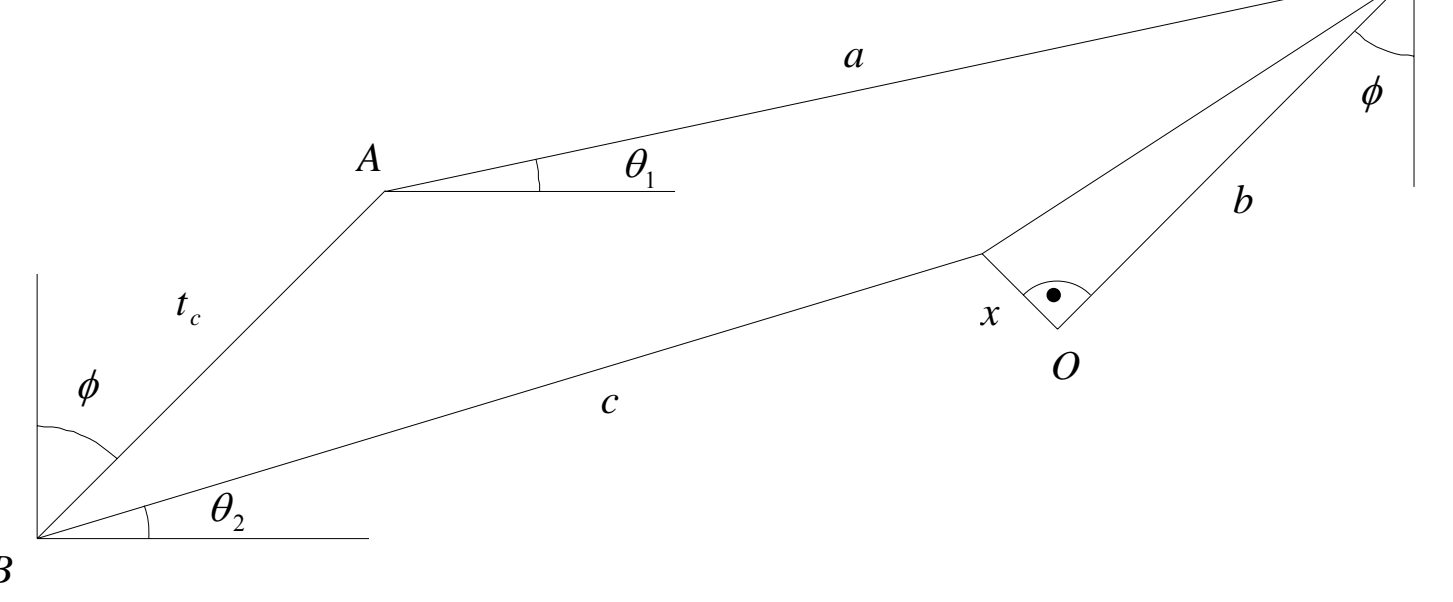

Figura 4.2. Detalle del espesor de viruta para el diente 1

Proyectando los segmentos $a$ y $c$ sobre $t_{c}$ se deduce que:

$$
t_{c}+a \cdot \cos \left(\frac{\pi}{2}-\theta_{1}-\phi\right)-b \cdot \cos \left(\frac{\pi}{2}-\theta_{2}-\phi\right)=b
$$

Desarrollando esta expresión se obtiene lo siguiente:

$$
t_{c}=b-a \cdot\left(\cos \theta_{1} \cdot \sin \phi+\sin \theta_{1} \cdot \cos \phi\right)+b \cdot\left(\cos \theta_{2} \cdot \sin \phi+\sin \theta_{2} \cdot \cos \phi\right)
$$


Sustituyendo los parámetros $a, b$ y $c$ por su valor según las expresiones (4.1), (4.2) y (4.3) se obtiene el espesor de viruta para el diente 1:

$$
\begin{aligned}
& t_{c}(1)=f_{z} \cdot \sin \phi+2 \cdot \rho \cdot \cos (\alpha-\phi-\lambda)+ \\
& \frac{1}{M} \cdot\left[1-\frac{K_{2}}{K_{1}} \cdot a_{p} \cdot\left(\frac{1}{2}-\frac{\alpha-\phi}{\delta}\right)\right] \cdot\left[\left(-F_{x}(1)+F_{x}(2)\right) \cdot \sin \phi+\left(-F_{y}(1)+F_{y}(2)\right) \cdot \cos \phi\right]
\end{aligned}
$$

Para el cálculo del espesor de viruta en el diente 2 se pueden utilizar las mismas expresiones que para el diente 1 cambiando los parámetros $a, b$ y $c$ por los siguientes valores:

$$
\begin{aligned}
& a=\left[1-\frac{K_{2}}{K_{1}} \cdot a_{p} \cdot\left(\frac{1}{2}-\frac{\alpha-\phi}{\delta}\right)\right] \cdot \frac{F_{T}(2)}{M} \\
& c=\left[1-\frac{K_{2}}{K_{1}} \cdot a_{p} \cdot\left(\frac{1}{2}-\frac{\alpha-\phi}{\delta}\right)\right] \cdot \frac{F_{T}(1)}{M} \\
& b=t_{c 0}=f_{z} \cdot \sin \phi-2 \cdot \rho \cdot \cos (\alpha-\phi-\lambda)
\end{aligned}
$$

Con ellos se obtiene la expresión para el espesor de viruta en el segundo diente:

$$
\begin{aligned}
& t_{c}(2)=f_{z} \cdot \sin \phi-2 \rho \cos (\alpha-\phi-\lambda)+ \\
& \frac{1}{M} \cdot\left[1-\frac{K_{2}}{K_{1}} \cdot a_{p} \cdot\left(\frac{1}{2}-\frac{\alpha-\phi}{\delta}\right)\right] \cdot\left[\left(F_{x}(1)-F_{x}(2)\right) \cdot \sin \phi+\left(F_{y}(1)-F_{y}(2)\right) \cdot \cos \phi\right]
\end{aligned}
$$

Ahora se pueden calcular las fuerzas mediante las expresiones que relacionaban las fuerzas con el espesor de viruta vistas en el capítulo anterior. Generalizando las expresiones (3.12) y (3.13) para los dos dientes se obtiene:

$$
d F_{x}(k)=\frac{D}{2 \cdot \tan \beta} \cdot\left[\begin{array}{l}
K_{t e} \cdot \cos \phi+K_{r e} \cdot \sin \phi+ \\
\left(K_{t c} \cdot \cos \phi+K_{r c} \cdot \sin \phi\right) \cdot t_{c}(k)
\end{array}\right] \cdot d \phi
$$




$$
d F_{y}(k)=\frac{D}{2 \cdot \tan \beta} \cdot\left[\begin{array}{l}
-K_{t e} \cdot \sin \phi+K_{r e} \cdot \cos \phi+ \\
\left(-K_{t c} \cdot \sin \phi+K_{r c} \cdot \cos \phi\right) \cdot t_{c}(k)
\end{array}\right] \cdot d \phi
$$

$$
k=1,2
$$

Sustituyendo los valores del espesor de viruta en estas expresiones se obtiene:

$$
d F_{x}(1)=\frac{D}{2 \cdot \tan \beta} \cdot\left[\begin{array}{l}
K_{t e} \cdot \cos \phi+K_{r e} \cdot \sin \phi+ \\
\left(K_{t c} \cdot \cos \phi+K_{r c} \cdot \sin \phi\right) \cdot \\
{\left[\begin{array}{l}
f_{z} \cdot \sin \phi+2 \cdot \rho \cdot \cos (\alpha-\phi-\lambda)+ \\
\frac{1}{M} \cdot\left[1-\frac{K_{2}}{K_{1}} \cdot a_{p} \cdot\left(\frac{1}{2}-\frac{\alpha-\phi}{\delta}\right)\right] \cdot\left[\begin{array}{l}
\left(-F_{x}(1)+F_{x}(2)\right) \cdot \sin \phi+ \\
\left(-F_{y}(1)+F_{y}(2)\right) \cdot \cos \phi
\end{array}\right]
\end{array}\right]}
\end{array}\right] \cdot d \phi
$$

$d F_{y}(1)=\frac{D}{2 \cdot \tan \beta} \cdot\left[\begin{array}{l}-K_{t e} \cdot \sin \phi+K_{r e} \cdot \cos \phi+ \\ \left(-K_{t c} \cdot \sin \phi+K_{r c} \cdot \cos \phi\right) \cdot \\ {\left[\begin{array}{l}f_{z} \cdot \sin \phi+2 \rho \cos (\alpha-\phi-\lambda)+ \\ \frac{1}{M} \cdot\left[1-\frac{K_{2}}{K_{1}} \cdot a_{p} \cdot\left(\frac{1}{2}-\frac{\alpha-\phi}{\delta}\right)\right] \cdot\left[\begin{array}{l}\left(-F_{x}(1)+F_{x}(2)\right) \cdot \sin \phi+ \\ \left(-F_{y}(1)+F_{y}(2)\right) \cdot \cos \phi\end{array}\right]\end{array}\right]}\end{array}\right] \cdot d \phi$

$$
d F_{x}(2)=\frac{D}{2 \cdot \tan \beta} \cdot\left[\begin{array}{l}
K_{t e} \cdot \cos \phi+K_{r e} \cdot \sin \phi+ \\
\left(K_{t c} \cdot \cos \phi+K_{r c} \cdot \sin \phi\right) \cdot \\
{\left[\begin{array}{l}
f_{z} \cdot \sin \phi-2 \rho \cos (\alpha-\phi-\lambda)+ \\
\frac{1}{M} \cdot\left[1-\frac{K_{2}}{K_{1}} \cdot a_{p} \cdot\left(\frac{1}{2}-\frac{\alpha-\phi}{\delta}\right)\right] \cdot\left[\begin{array}{l}
\left(F_{x}(1)-F_{x}(2)\right) \cdot \sin \phi+ \\
\left(F_{y}(1)-F_{y}(2)\right) \cdot \cos \phi
\end{array}\right]
\end{array}\right]}
\end{array}\right] \cdot d \phi
$$




$$
d F_{y}(2)=\frac{D}{2 \cdot \tan \beta} \cdot\left[\begin{array}{l}
-K_{t e} \cdot \sin \phi+K_{r e} \cdot \cos \phi+ \\
\left(-K_{t c} \cdot \sin \phi+K_{r c} \cdot \cos \phi\right) \cdot \\
{\left[\begin{array}{l}
f_{z} \cdot \sin \phi-2 \rho \cos (\alpha-\phi-\lambda)+ \\
\frac{1}{M} \cdot\left[1-\frac{K_{2}}{K_{1}} \cdot a_{p} \cdot\left(\frac{1}{2}-\frac{\alpha-\phi}{\delta}\right)\right] \cdot\left[\begin{array}{l}
\left(F_{x}(1)-F_{x}(2)\right) \cdot \sin \phi+ \\
\left(F_{y}(1)-F_{y}(2)\right) \cdot \cos \phi
\end{array}\right]
\end{array}\right]}
\end{array}\right] \cdot d \phi
$$

Estas expresiones pueden ser integradas considerando las fuerzas de corte constantes a lo largo del filo, es decir, cuando varía el ángulo $\phi$. Realizando la integración y reordenando términos se obtiene: 


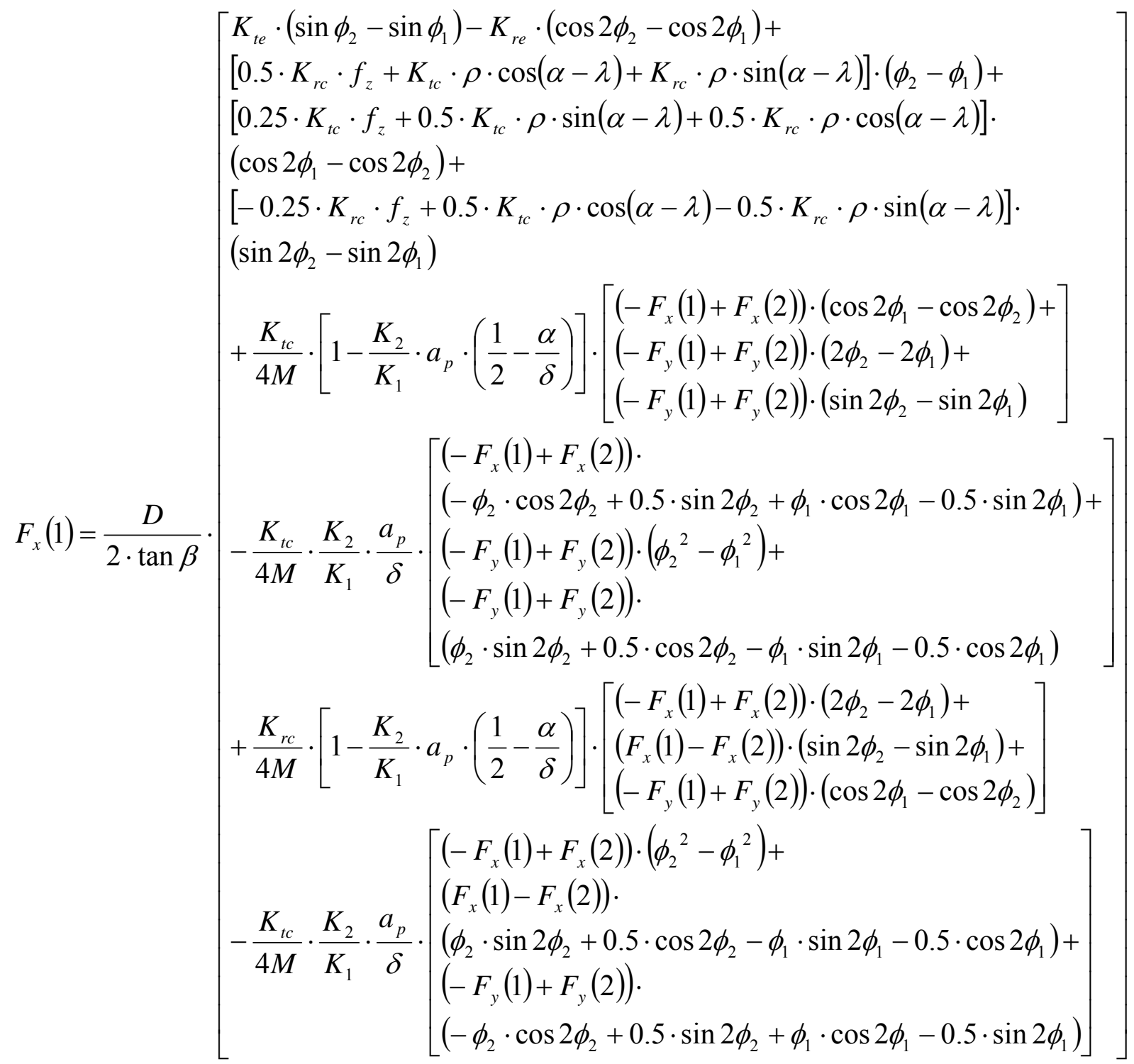




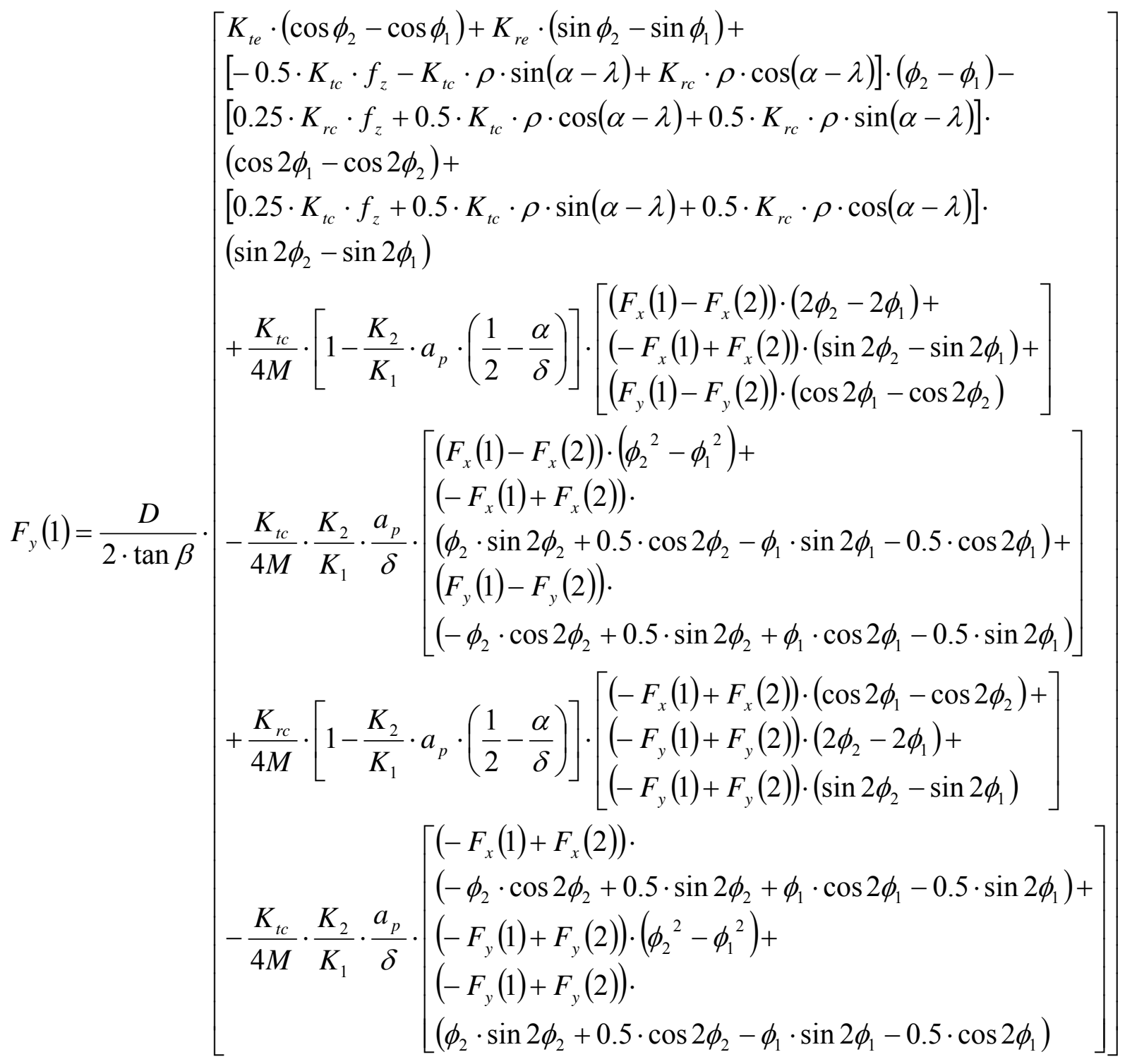




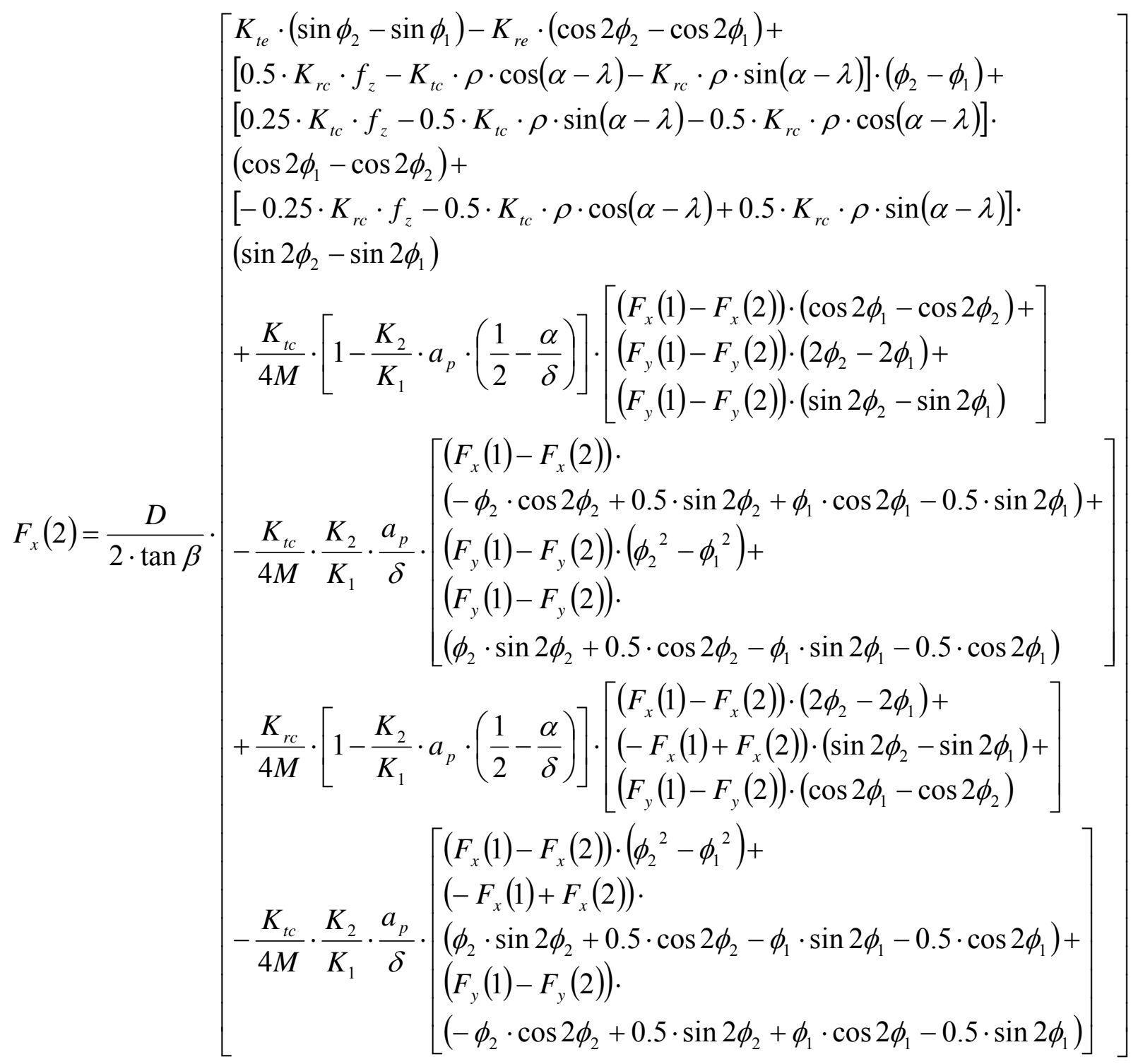




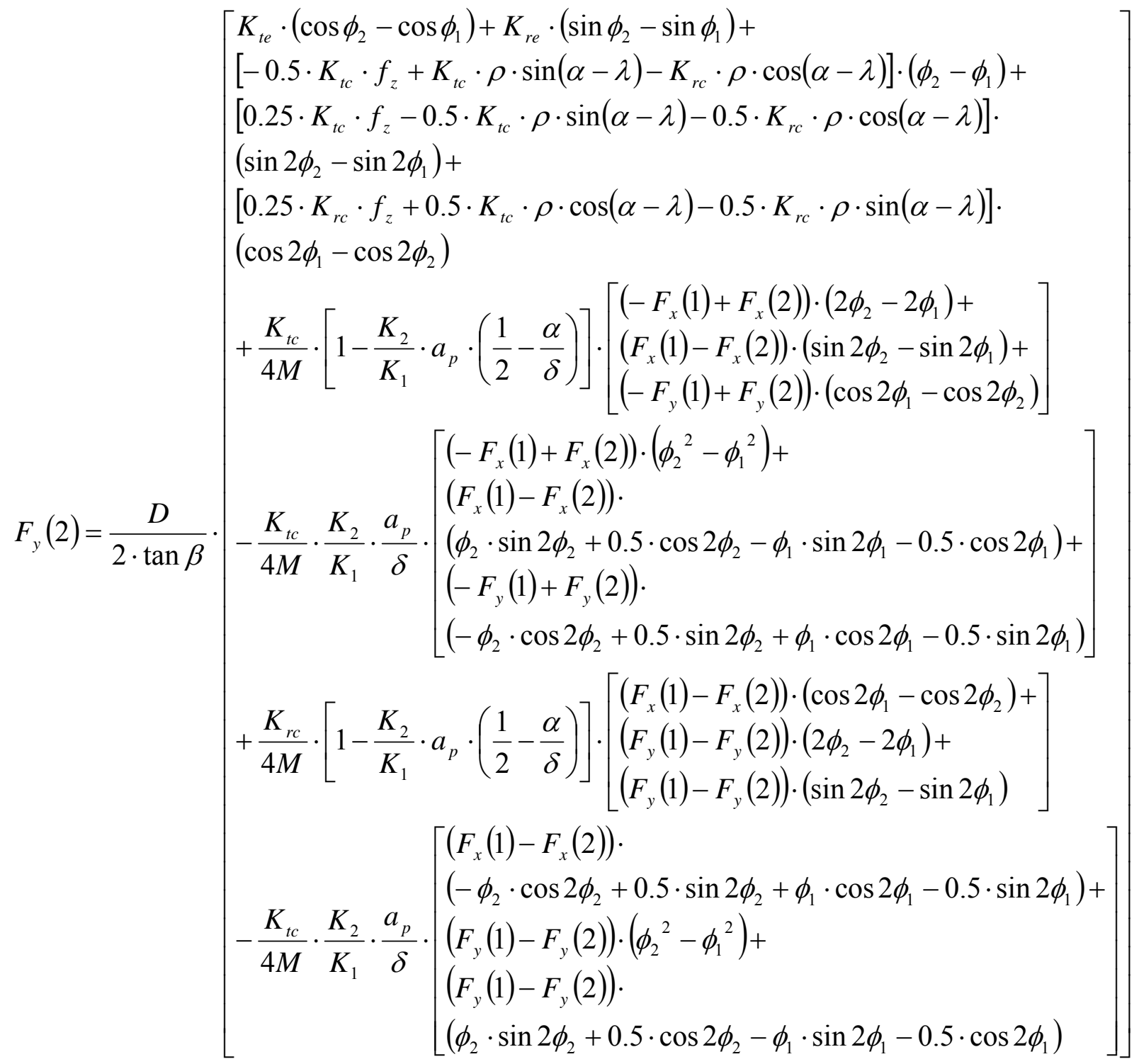

Se puede observar que estas expresiones constituyen un sistema de ecuaciones lineales en el que las incógnitas son las componentes de las fuerzas en los dos filos, de tal manera que el sistema puede expresarse matricialmente de la manera siguiente:

$$
\vec{F}=A \cdot \vec{F}+\vec{b}
$$

Donde los componentes del vector $\boldsymbol{F}$ son los componentes de las fuerzas de corte, los de la matriz $A$ son los coeficientes que acompañan a los componentes de las fuerzas y los del vector $\boldsymbol{b}$ son los términos independientes: 


$$
\begin{gathered}
\vec{F}=\left[\begin{array}{l}
F_{x}(1) \\
F_{y}(1) \\
F_{x}(2) \\
F_{y}(2)
\end{array}\right] \quad(4.22) \\
A=\left[\begin{array}{llll}
a_{11} & a_{12} & a_{13} & a_{14} \\
a_{21} & a_{22} & a_{23} & a_{24} \\
a_{31} & a_{32} & a_{33} & a_{34} \\
a_{41} & a_{42} & a_{43} & a_{44}
\end{array}\right] \\
\vec{b}=\left[\begin{array}{l}
b_{1} \\
b_{2} \\
b_{3} \\
b_{4}
\end{array}\right]
\end{gathered}
$$

Los coeficientes $a_{i j}$ de la matriz $A$ se pueden extraer de las expresiones (4.17)-(4.20), al igual que los componentes $b_{i}$ del vector $\boldsymbol{b}$ :

$$
a_{11}=\frac{D}{8 \cdot M \cdot \tan \beta} \cdot\left[\begin{array}{l}
K_{t c} \cdot\left[1-\frac{K_{2}}{K_{1}} \cdot a_{p} \cdot\left(\frac{1}{2}-\frac{\alpha}{\delta}\right)\right] \cdot\left(\cos 2 \phi_{2}-\cos 2 \phi_{1}\right) \\
-K_{t c} \cdot \frac{K_{2}}{K_{1}} \cdot \frac{a_{p}}{\delta} \cdot\left(\begin{array}{l}
\left.\phi_{2} \cdot \cos 2 \phi_{2}-0.5 \cdot \sin 2 \phi_{2}-\right) \\
\phi_{1} \cdot \cos 2 \phi_{1}+0.5 \cdot \sin 2 \phi_{1}
\end{array}\right)+ \\
K_{r c} \cdot\left[1-\frac{K_{2}}{K_{1}} \cdot a_{p} \cdot\left(\frac{1}{2}-\frac{\alpha}{\delta}\right)\right] \cdot\left[\left(2 \phi_{1}-2 \phi_{2}\right)+\left(\sin 2 \phi_{2}-\sin 2 \phi_{1}\right)\right] \\
-K_{r c} \cdot \frac{K_{2}}{K_{1}} \cdot \frac{a_{p}}{\delta} \cdot\left[\left(\phi_{1}^{2}-\phi_{2}^{2}\right)+\left(\begin{array}{l}
\left.\phi_{2} \cdot \sin 2 \phi_{2}+0.5 \cdot \cos 2 \phi_{2}-\right) \\
\phi_{1} \cdot \sin 2 \phi_{1}-0.5 \cdot \cos 2 \phi_{1}
\end{array}\right)\right]
\end{array}\right]
$$




$$
a_{12}=\frac{D}{8 \cdot M \cdot \tan \beta} \cdot\left[\begin{array}{l}
K_{t c} \cdot\left[1-\frac{K_{2}}{K_{1}} \cdot a_{p} \cdot\left(\frac{1}{2}-\frac{\alpha}{\delta}\right)\right] \cdot\left[\left(2 \phi_{1}-2 \phi_{2}\right)+\left(\sin 2 \phi_{1}-\sin 2 \phi_{2}\right)\right] \\
-K_{t c} \cdot \frac{K_{2}}{K_{1}} \cdot \frac{a_{p}}{\delta} \cdot\left[\left(\phi_{1}^{2}-\phi_{2}^{2}\right)+\left(\begin{array}{l}
\left.-\phi_{2} \cdot \sin 2 \phi_{2}-0.5 \cdot \cos 2 \phi_{2}+\right) \\
\phi_{1} \cdot \sin 2 \phi_{1}+0.5 \cdot \cos 2 \phi_{1}
\end{array}\right)\right]+ \\
K_{r c} \cdot\left[1-\frac{K_{2}}{K_{1}} \cdot a_{p} \cdot\left(\frac{1}{2}-\frac{\alpha}{\delta}\right)\right] \cdot\left(\cos 2 \phi_{2}-\cos 2 \phi_{1}\right)
\end{array}\right]
$$

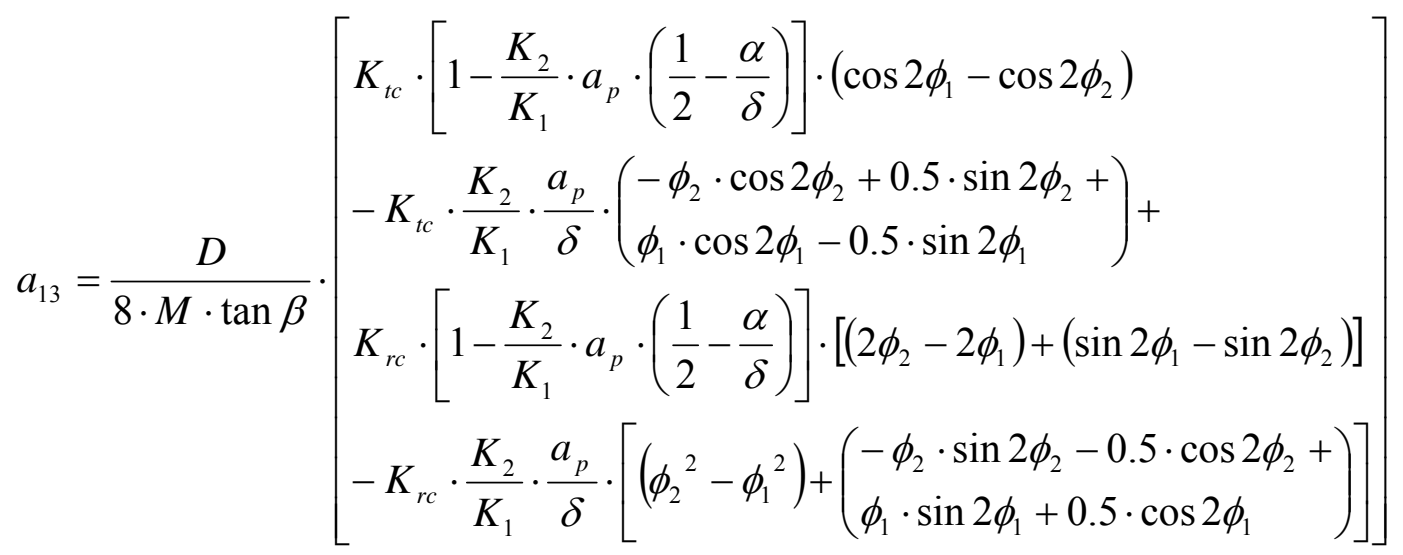

$$
a_{14}=\frac{D}{8 \cdot M \cdot \tan \beta} \cdot\left[\begin{array}{l}
K_{t c} \cdot\left[1-\frac{K_{2}}{K_{1}} \cdot a_{p} \cdot\left(\frac{1}{2}-\frac{\alpha}{\delta}\right)\right] \cdot\left[\left(2 \phi_{2}-2 \phi_{1}\right)+\left(\sin 2 \phi_{2}-\sin 2 \phi_{1}\right)\right] \\
-K_{t c} \cdot \frac{K_{2}}{K_{1}} \cdot \frac{a_{p}}{\delta} \cdot\left[\left(\phi_{2}^{2}-\phi_{1}^{2}\right)+\left(\begin{array}{l}
\left.\phi_{2} \cdot \sin 2 \phi_{2}+0.5 \cdot \cos 2 \phi_{2}-\right) \\
\phi_{1} \cdot \sin 2 \phi_{1}-0.5 \cdot \cos 2 \phi_{1}
\end{array}\right)\right]+ \\
K_{r c} \cdot\left[1-\frac{K_{2}}{K_{1}} \cdot a_{p} \cdot\left(\frac{1}{2}-\frac{\alpha}{\delta}\right)\right] \cdot\left(\cos 2 \phi_{1}-\cos 2 \phi_{2}\right) \\
-K_{r c} \cdot \frac{K_{2}}{K_{1}} \cdot \frac{a_{p}}{\delta} \cdot\left(\begin{array}{l}
-\phi_{2} \cdot \cos 2 \phi_{2}+0.5 \cdot \sin 2 \phi_{2}+ \\
\phi_{1} \cdot \cos 2 \phi_{1}-0.5 \cdot \sin 2 \phi_{1}
\end{array}\right)
\end{array}\right]
$$




$$
a_{21}=\frac{D}{8 \cdot M \cdot \tan \beta} \cdot\left[\begin{array}{l}
K_{t c} \cdot\left[1-\frac{K_{2}}{K_{1}} \cdot a_{p} \cdot\left(\frac{1}{2}-\frac{\alpha}{\delta}\right)\right] \cdot\left[\left(2 \phi_{2}-2 \phi_{1}\right)+\left(\sin 2 \phi_{1}-\sin 2 \phi_{2}\right)\right] \\
-K_{t c} \cdot \frac{K_{2}}{K_{1}} \cdot \frac{a_{p}}{\delta} \cdot\left[\left(\phi_{2}^{2}-\phi_{1}^{2}\right)+\left(\begin{array}{l}
\left.-\phi_{2} \cdot \sin 2 \phi_{2}-0.5 \cdot \cos 2 \phi_{2}+\right) \\
\phi_{1} \cdot \sin 2 \phi_{1}+0.5 \cdot \cos 2 \phi_{1}
\end{array}\right)\right]+ \\
K_{r c} \cdot\left[1-\frac{K_{2}}{K_{1}} \cdot a_{p} \cdot\left(\frac{1}{2}-\frac{\alpha}{\delta}\right)\right] \cdot\left(\cos 2 \phi_{2}-\cos 2 \phi_{1}\right) \\
-K_{r c} \cdot \frac{K_{2}}{K_{1}} \cdot \frac{a_{p}}{\delta} \cdot\left(\begin{array}{l}
\phi_{2} \cdot \cos 2 \phi_{2}-0.5 \cdot \sin 2 \phi_{2}- \\
\phi_{1} \cdot \cos 2 \phi_{1}+0.5 \cdot \sin 2 \phi_{1}
\end{array}\right)
\end{array}\right]
$$

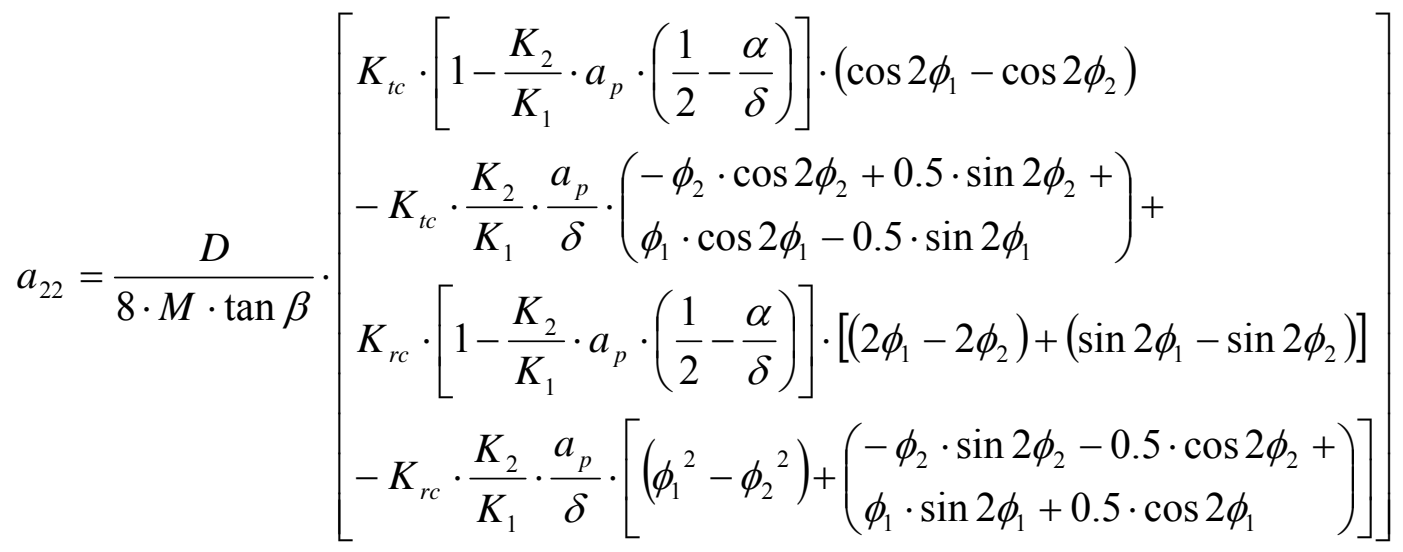

$$
a_{23}=\frac{D}{8 \cdot M \cdot \tan \beta} \cdot\left[\begin{array}{l}
K_{t c} \cdot\left[1-\frac{K_{2}}{K_{1}} \cdot a_{p} \cdot\left(\frac{1}{2}-\frac{\alpha}{\delta}\right)\right] \cdot\left[\left(2 \phi_{1}-2 \phi_{2}\right)+\left(\sin 2 \phi_{2}-\sin 2 \phi_{1}\right)\right] \\
-K_{t c} \cdot \frac{K_{2}}{K_{1}} \cdot \frac{a_{p}}{\delta} \cdot\left[\left(\phi_{1}^{2}-\phi_{2}^{2}\right)+\left(\begin{array}{l}
\left.\phi_{2} \cdot \sin 2 \phi_{2}+0.5 \cdot \cos 2 \phi_{2}-\right) \\
\phi_{1} \cdot \sin 2 \phi_{1}-0.5 \cdot \cos 2 \phi_{1}
\end{array}\right)\right]+ \\
K_{r c} \cdot\left[1-\frac{K_{2}}{K_{1}} \cdot a_{p} \cdot\left(\frac{1}{2}-\frac{\alpha}{\delta}\right)\right] \cdot\left(\cos 2 \phi_{1}-\cos 2 \phi_{2}\right) \\
-K_{r c} \cdot \frac{K_{2}}{K_{1}} \cdot \frac{a_{p}}{\delta} \cdot\left(\begin{array}{l}
-\phi_{2} \cdot \cos 2 \phi_{2}+0.5 \cdot \sin 2 \phi_{2}+ \\
\phi_{1} \cdot \cos 2 \phi_{1}-0.5 \cdot \sin 2 \phi_{1}
\end{array}\right)
\end{array}\right]
$$




$$
a_{24}=\frac{D}{8 \cdot M \cdot \tan \beta} \cdot\left[\begin{array}{l}
K_{t c} \cdot\left[1-\frac{K_{2}}{K_{1}} \cdot a_{p} \cdot\left(\frac{1}{2}-\frac{\alpha}{\delta}\right)\right] \cdot\left(\cos 2 \phi_{2}-\cos 2 \phi_{1}\right) \\
-K_{t c} \cdot \frac{K_{2}}{K_{1}} \cdot \frac{a_{p}}{\delta} \cdot\left(\begin{array}{l}
\phi_{2} \cdot \cos 2 \phi_{2}-0.5 \cdot \sin 2 \phi_{2}- \\
\phi_{1} \cdot \cos 2 \phi_{1}+0.5 \cdot \sin 2 \phi_{1}
\end{array}\right)+ \\
K_{r c} \cdot\left[1-\frac{K_{2}}{K_{1}} \cdot a_{p} \cdot\left(\frac{1}{2}-\frac{\alpha}{\delta}\right)\right] \cdot\left[\left(2 \phi_{2}-2 \phi_{1}\right)+\left(\sin 2 \phi_{2}-\sin 2 \phi_{1}\right)\right] \\
-K_{r c} \cdot \frac{K_{2}}{K_{1}} \cdot \frac{a_{p}}{\delta} \cdot\left[\left(\phi_{2}{ }^{2}-\phi_{1}{ }^{2}\right)+\left(\begin{array}{l}
\phi_{2} \cdot \sin 2 \phi_{2}+0.5 \cdot \cos 2 \phi_{2}- \\
\phi_{1} \cdot \sin 2 \phi_{1}-0.5 \cdot \cos 2 \phi_{1}
\end{array}\right)\right.
\end{array}\right]
$$

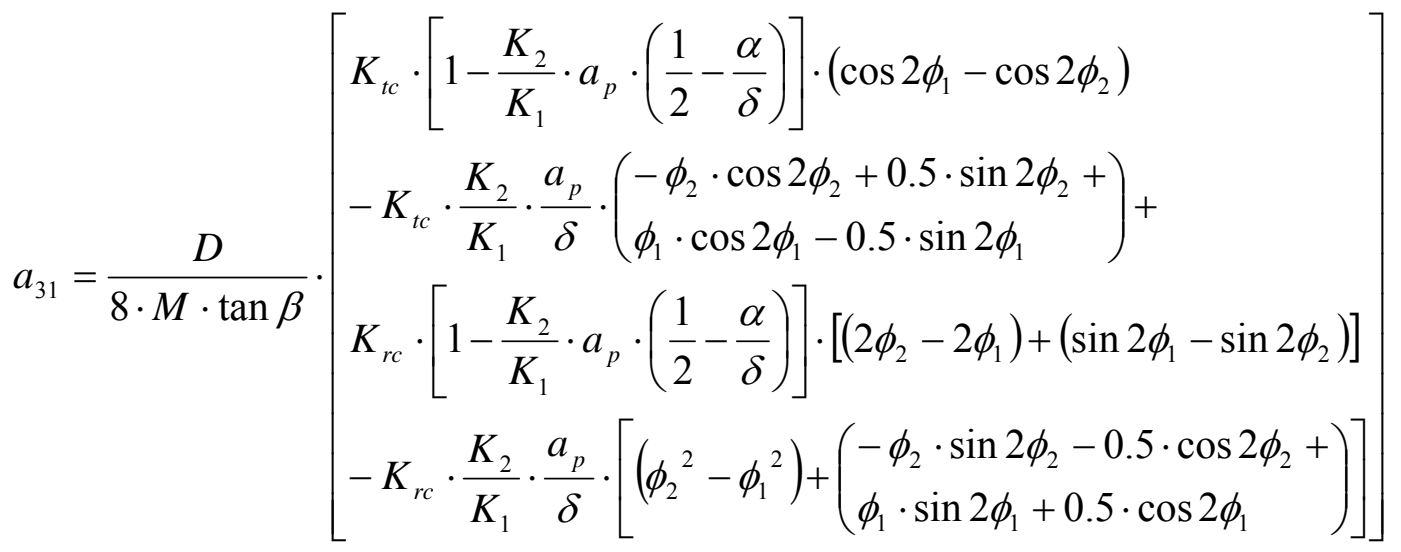

$$
a_{32}=\frac{D}{8 \cdot M \cdot \tan \beta} \cdot\left[\begin{array}{l}
K_{t c} \cdot\left[1-\frac{K_{2}}{K_{1}} \cdot a_{p} \cdot\left(\frac{1}{2}-\frac{\alpha}{\delta}\right)\right] \cdot\left[\left(2 \phi_{2}-2 \phi_{1}\right)+\left(\sin 2 \phi_{2}-\sin 2 \phi_{1}\right)\right] \\
-K_{t c} \cdot \frac{K_{2}}{K_{1}} \cdot \frac{a_{p}}{\delta} \cdot\left[\left(\phi_{2}{ }^{2}-\phi_{1}^{2}\right)+\left(\begin{array}{l}
\left.\phi_{2} \cdot \sin 2 \phi_{2}+0.5 \cdot \cos 2 \phi_{2}-\right) \\
\phi_{1} \cdot \sin 2 \phi_{1}-0.5 \cdot \cos 2 \phi_{1}
\end{array}\right)\right]+ \\
K_{r c} \cdot\left[1-\frac{K_{2}}{K_{1}} \cdot a_{p} \cdot\left(\frac{1}{2}-\frac{\alpha}{\delta}\right)\right] \cdot\left(\cos 2 \phi_{1}-\cos 2 \phi_{2}\right) \\
-K_{r c} \cdot \frac{K_{2}}{K_{1}} \cdot \frac{a_{p}}{\delta} \cdot\left(\begin{array}{l}
-\phi_{2} \cdot \cos 2 \phi_{2}+0.5 \cdot \sin 2 \phi_{2}+ \\
\phi_{1} \cdot \cos 2 \phi_{1}-0.5 \cdot \sin 2 \phi_{1}
\end{array}\right)
\end{array}\right]
$$




$$
a_{33}=\frac{D}{8 \cdot M \cdot \tan \beta} \cdot\left[\begin{array}{l}
K_{t c} \cdot\left[1-\frac{K_{2}}{K_{1}} \cdot a_{p} \cdot\left(\frac{1}{2}-\frac{\alpha}{\delta}\right)\right] \cdot\left(\cos 2 \phi_{2}-\cos 2 \phi_{1}\right) \\
-K_{t c} \cdot \frac{K_{2}}{K_{1}} \cdot \frac{a_{p}}{\delta} \cdot\left(\begin{array}{l}
\left.\phi_{2} \cdot \cos 2 \phi_{2}-0.5 \cdot \sin 2 \phi_{2}-\right) \\
\phi_{1} \cdot \cos 2 \phi_{1}+0.5 \cdot \sin 2 \phi_{1}
\end{array}\right)+ \\
K_{r c} \cdot\left[1-\frac{K_{2}}{K_{1}} \cdot a_{p} \cdot\left(\frac{1}{2}-\frac{\alpha}{\delta}\right)\right] \cdot\left[\left(2 \phi_{1}-2 \phi_{2}\right)+\left(\sin 2 \phi_{2}-\sin 2 \phi_{1}\right)\right]
\end{array}\right]
$$

$$
a_{34}=\frac{D}{8 \cdot M \cdot \tan \beta} \cdot\left[\begin{array}{l}
K_{t c} \cdot\left[1-\frac{K_{2}}{K_{1}} \cdot a_{p} \cdot\left(\frac{1}{2}-\frac{\alpha}{\delta}\right)\right] \cdot\left[\left(2 \phi_{1}-2 \phi_{2}\right)+\left(\sin 2 \phi_{1}-\sin 2 \phi_{2}\right)\right] \\
-K_{t c} \cdot \frac{K_{2}}{K_{1}} \cdot \frac{a_{p}}{\delta} \cdot\left[\left(\phi_{1}^{2}-\phi_{2}^{2}\right)+\left(\begin{array}{l}
\left.-\phi_{2} \cdot \sin 2 \phi_{2}-0.5 \cdot \cos 2 \phi_{2}+\right) \\
\phi_{1} \cdot \sin 2 \phi_{1}+0.5 \cdot \cos 2 \phi_{1}
\end{array}\right)\right]+ \\
K_{r c} \cdot\left[1-\frac{K_{2}}{K_{1}} \cdot a_{p} \cdot\left(\frac{1}{2}-\frac{\alpha}{\delta}\right)\right] \cdot\left(\cos 2 \phi_{2}-\cos 2 \phi_{1}\right) \\
-K_{r c} \cdot \frac{K_{2}}{K_{1}} \cdot \frac{a_{p}}{\delta} \cdot\left(\begin{array}{l}
\phi_{2} \cdot \cos 2 \phi_{2}-0.5 \cdot \sin 2 \phi_{2}- \\
\phi_{1} \cdot \cos 2 \phi_{1}+0.5 \cdot \sin 2 \phi_{1}
\end{array}\right)
\end{array}\right]
$$

$$
a_{41}=\frac{D}{8 \cdot M \cdot \tan \beta} \cdot\left[\begin{array}{l}
K_{t c} \cdot\left[1-\frac{K_{2}}{K_{1}} \cdot a_{p} \cdot\left(\frac{1}{2}-\frac{\alpha}{\delta}\right)\right] \cdot\left[\left(2 \phi_{1}-2 \phi_{2}\right)+\left(\sin 2 \phi_{2}-\sin 2 \phi_{1}\right)\right] \\
-K_{t c} \cdot \frac{K_{2}}{K_{1}} \cdot \frac{a_{p}}{\delta} \cdot\left[\left(\phi_{1}^{2}-\phi_{2}{ }^{2}\right)+\left(\begin{array}{l}
\left.\phi_{2} \cdot \sin 2 \phi_{2}+0.5 \cdot \cos 2 \phi_{2}-\right) \\
\phi_{1} \cdot \sin 2 \phi_{1}-0.5 \cdot \cos 2 \phi_{1}
\end{array}\right)\right]+ \\
K_{r c} \cdot\left[1-\frac{K_{2}}{K_{1}} \cdot a_{p} \cdot\left(\frac{1}{2}-\frac{\alpha}{\delta}\right)\right] \cdot\left(\cos 2 \phi_{1}-\cos 2 \phi_{2}\right) \\
-K_{r c} \cdot \frac{K_{2}}{K_{1}} \cdot \frac{a_{p}}{\delta} \cdot\left(\begin{array}{l}
-\phi_{2} \cdot \cos 2 \phi_{2}+0.5 \cdot \sin 2 \phi_{2}+ \\
\phi_{1} \cdot \cos 2 \phi_{1}-0.5 \cdot \sin 2 \phi_{1}
\end{array}\right)
\end{array}\right]
$$




$$
a_{42}=\frac{D}{8 \cdot M \cdot \tan \beta} \cdot\left[\begin{array}{l}
K_{t c} \cdot\left[1-\frac{K_{2}}{K_{1}} \cdot a_{p} \cdot\left(\frac{1}{2}-\frac{\alpha}{\delta}\right)\right] \cdot\left(\cos 2 \phi_{2}-\cos 2 \phi_{1}\right) \\
-K_{t c} \cdot \frac{K_{2}}{K_{1}} \cdot \frac{a_{p}}{\delta} \cdot\left(\begin{array}{l}
\phi_{2} \cdot \cos 2 \phi_{2}-0.5 \cdot \sin 2 \phi_{2}- \\
\phi_{1} \cdot \cos 2 \phi_{1}+0.5 \cdot \sin 2 \phi_{1}
\end{array}\right)+ \\
K_{r c} \cdot\left[1-\frac{K_{2}}{K_{1}} \cdot a_{p} \cdot\left(\frac{1}{2}-\frac{\alpha}{\delta}\right)\right] \cdot\left[\left(2 \phi_{2}-2 \phi_{1}\right)+\left(\sin 2 \phi_{2}-\sin 2 \phi_{1}\right)\right] \\
-K_{r c} \cdot \frac{K_{2}}{K_{1}} \cdot \frac{a_{p}}{\delta} \cdot\left[\left(\phi_{2}^{2}-\phi_{1}^{2}\right)+\left(\begin{array}{l}
\phi_{2} \cdot \sin 2 \phi_{2}+0.5 \cdot \cos 2 \phi_{2}- \\
\phi_{1} \cdot \sin 2 \phi_{1}-0.5 \cdot \cos 2 \phi_{1}
\end{array}\right)\right]
\end{array}\right]
$$

$$
a_{43}=\frac{D}{8 \cdot M \cdot \tan \beta} \cdot\left[\begin{array}{l}
K_{t c} \cdot\left[1-\frac{K_{2}}{K_{1}} \cdot a_{p} \cdot\left(\frac{1}{2}-\frac{\alpha}{\delta}\right)\right] \cdot\left[\left(2 \phi_{2}-2 \phi_{1}\right)+\left(\sin 2 \phi_{1}-\sin 2 \phi_{2}\right)\right] \\
-K_{t c} \cdot \frac{K_{2}}{K_{1}} \cdot \frac{a_{p}}{\delta} \cdot\left[\left(\phi_{2}^{2}-\phi_{1}^{2}\right)+\left(\begin{array}{l}
\left.-\phi_{2} \cdot \sin 2 \phi_{2}-0.5 \cdot \cos 2 \phi_{2}+\right) \\
\phi_{1} \cdot \sin 2 \phi_{1}+0.5 \cdot \cos 2 \phi_{1}
\end{array}\right)\right]+ \\
K_{r c} \cdot\left[1-\frac{K_{2}}{K_{1}} \cdot a_{p} \cdot\left(\frac{1}{2}-\frac{\alpha}{\delta}\right)\right] \cdot\left(\cos 2 \phi_{2}-\cos 2 \phi_{1}\right) \\
-K_{r c} \cdot \frac{K_{2}}{K_{1}} \cdot \frac{a_{p}}{\delta} \cdot\left(\begin{array}{l}
\phi_{2} \cdot \cos 2 \phi_{2}-0.5 \cdot \sin 2 \phi_{2}- \\
\phi_{1} \cdot \cos 2 \phi_{1}+0.5 \cdot \sin 2 \phi_{1}
\end{array}\right)
\end{array}\right]
$$

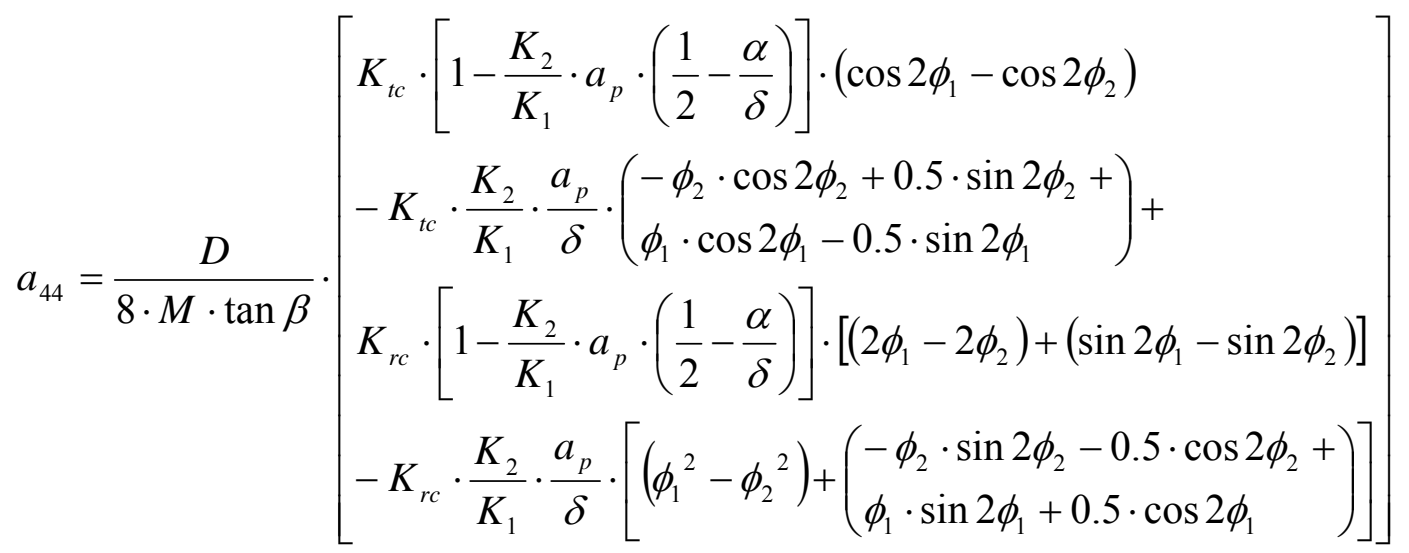

$$
b_{1}=\frac{D}{8 \cdot \tan \beta} \cdot\left[\begin{array}{l}
4 \cdot K_{t e} \cdot\left(\sin \phi_{2}-\sin \phi_{1}\right)-4 \cdot K_{r e} \cdot\left(\cos \phi_{2}-\cos \phi_{1}\right)+ \\
{\left[\begin{array}{l}
2 \cdot K_{r c} \cdot f_{z}+4 \cdot K_{t c} \cdot \rho \cdot \cos (\alpha-\lambda)+ \\
4 \cdot K_{r c} \cdot \rho \cdot \sin (\alpha-\lambda)
\end{array}\right] \cdot\left(\phi_{2}-\phi_{1}\right)+} \\
{\left[\begin{array}{l}
\left.K_{t c} \cdot f_{z}+2 \cdot K_{t c} \cdot \rho \cdot \sin (\alpha-\lambda)+\right] \\
2 \cdot K_{r c} \cdot \rho \cdot \cos (\alpha-\lambda)
\end{array}\right] \cdot\left(\cos 2 \phi_{1}-\cos 2 \phi_{2}\right)+} \\
{\left[\begin{array}{l}
-K_{r c} \cdot f_{z}+2 \cdot K_{t c} \cdot \rho \cdot \cos (\alpha-\lambda)- \\
2 \cdot K_{r c} \cdot \rho \cdot \sin (\alpha-\lambda)
\end{array}\right] \cdot\left(\sin 2 \phi_{2}-\sin 2 \phi_{1}\right)}
\end{array}\right]
$$


$b_{2}=\frac{D}{8 \cdot \tan \beta} \cdot\left[\begin{array}{l}4 \cdot K_{t e} \cdot\left(\cos \phi_{2}-\cos \phi_{1}\right)+4 \cdot K_{r e} \cdot\left(\sin \phi_{2}-\sin \phi_{1}\right)+ \\ {\left[\begin{array}{l}-2 \cdot K_{t c} \cdot f_{z}-4 \cdot K_{t c} \cdot \rho \cdot \sin (\alpha-\lambda)+ \\ 4 \cdot K_{r c} \cdot \rho \cdot \cos (\alpha-\lambda)\end{array}\right] \cdot\left(\phi_{2}-\phi_{1}\right)+} \\ {\left[\begin{array}{l}\left.K_{r c} \cdot f_{z}-2 \cdot K_{t c} \cdot \rho \cdot \cos (\alpha-\lambda)+\right] \\ 2 \cdot K_{r c} \cdot \rho \cdot \sin (\alpha-\lambda)\end{array}\right] \cdot\left(\cos 2 \phi_{1}-\cos 2 \phi_{2}\right)+} \\ {\left[\begin{array}{l}K_{t c} \cdot f_{z}+2 \cdot K_{t c} \cdot \rho \cdot \sin (\alpha-\lambda)+ \\ 2 \cdot K_{r c} \cdot \rho \cdot \cos (\alpha-\lambda)\end{array}\right] \cdot\left(\sin 2 \phi_{2}-\sin 2 \phi_{1}\right)}\end{array}\right]$

$b_{3}=\frac{D}{8 \cdot \tan \beta} \cdot\left[\begin{array}{l}4 \cdot K_{t e} \cdot\left(\sin \phi_{2}-\sin \phi_{1}\right)-4 \cdot K_{r e} \cdot\left(\cos \phi_{2}-\cos \phi_{1}\right)+ \\ {\left[\begin{array}{l}2 \cdot K_{r c} \cdot f_{z}-4 \cdot K_{t c} \cdot \rho \cdot \cos (\alpha-\lambda)- \\ 4 \cdot K_{r c} \cdot \rho \cdot \sin (\alpha-\lambda)\end{array}\right] \cdot\left(\phi_{2}-\phi_{1}\right)+} \\ {\left[\begin{array}{l}K_{t c} \cdot f_{z}-2 \cdot K_{t c} \cdot \rho \cdot \sin (\alpha-\lambda)- \\ 2 \cdot K_{r c} \cdot \rho \cdot \cos (\alpha-\lambda)\end{array}\right] \cdot\left(\cos 2 \phi_{1}-\cos 2 \phi_{2}\right)+} \\ {\left[\begin{array}{l}-K_{r c} \cdot f_{z}-2 \cdot K_{t c} \cdot \rho \cdot \cos (\alpha-\lambda)+ \\ 2 \cdot K_{r c} \cdot \rho \cdot \sin (\alpha-\lambda)\end{array}\right] \cdot\left(\sin 2 \phi_{2}-\sin 2 \phi_{1}\right)}\end{array}\right]$

$b_{4}=\frac{D}{8 \cdot \tan \beta} \cdot\left[\begin{array}{l}4 \cdot K_{t e} \cdot\left(\cos \phi_{2}-\cos \phi_{1}\right)+4 \cdot K_{r e} \cdot\left(\sin \phi_{2}-\sin \phi_{1}\right)+ \\ {\left[\begin{array}{l}-2 \cdot K_{t c} \cdot f_{z}+4 \cdot K_{t c} \cdot \rho \cdot \sin (\alpha-\lambda)- \\ 4 \cdot K_{r c} \cdot \rho \cdot \cos (\alpha-\lambda)\end{array}\right] \cdot\left(\phi_{2}-\phi_{1}\right)+} \\ {\left[\begin{array}{l}K_{r c} \cdot f_{z}-2 \cdot K_{r c} \cdot \rho \cdot \sin (\alpha-\lambda)+ \\ 2 \cdot K_{t c} \cdot \rho \cdot \cos (\alpha-\lambda)\end{array}\right] \cdot\left(\cos 2 \phi_{1}-\cos 2 \phi_{2}\right)+} \\ {\left[\begin{array}{l}K_{t c} \cdot f_{z}-2 \cdot K_{r c} \cdot \rho \cdot \cos (\alpha-\lambda)- \\ 2 \cdot K_{t c} \cdot \rho \cdot \sin (\alpha-\lambda)\end{array}\right] \cdot\left(\sin 2 \phi_{2}-\sin 2 \phi_{1}\right)}\end{array}\right]$

Utilizando estos coeficientes el sistema puede ser resuelto despejando el vector $\boldsymbol{F}$ de la expresión (83):

$$
\vec{F}=(I-A)^{-1} \cdot \vec{b}
$$

\subsubsection{Efecto tamaño}

Cuando el espesor de viruta es muy pequeño comparado con el radio de redondeo del filo, el filo de corte no puede ser considerado como una línea, sino como una superficie 
redondeada. Esto ocurre en todos los procesos de microfresado debido a que el radio del filo no se puede reducir tanto como se quiera, ya que existe la limitación que impone por un lado el procedimiento de fabricación de la fresa y por otro la robustez de la herramienta [108].

Este factor provocará que exista un componente importante de aplastamiento o recalcado en la fuerza del proceso, como se ha reflejado en el modelo. Por otro lado, el redondeo del filo hará que en algunos casos no exista formación de viruta, sino sólo fenómeno de aplastamiento. Esto se conoce con el nombre de efecto tamaño, en el cual se han realizado trabajos de investigación como [70]. Estos autores indican que la viruta debe tener un espesor mínimo para que sea arrancada, por debajo de ese valor no existirá tensión cortante suficiente y se producirá sólo recalcado. Este valor mínimo del espesor de viruta depende del radio de redondeo del filo.

En consecuencia, los filos no empiezan a cortar hasta que el espesor de viruta alcanza un cierto valor en el fresado en oposición. Análogamente, en el fresado en concordancia el diente dejará de cortar cuando el espesor de viruta se haga menor al valor crítico. Este valor puede calcularse multiplicando el radio del filo por una constante de proporcionalidad experimental $\lambda_{c}$, como se indica en la siguiente expresión:

$$
t_{c \min }=\lambda_{c} \cdot r_{e}
$$

Por tanto, con el valor crítico se pueden calcular los ángulos de entrada y salida del corte.

En el fresado en oposición el corte no comenzará en el ángulo 0, aun en condiciones de fresado ideal, sino que existirá un ángulo por debajo del cual no se alcanza el espesor de viruta mínimo. Este ángulo puede calcularse para fresado en oposición a partir de la expresión (3.14):

$$
\alpha_{a c}=\arcsin \frac{\lambda_{c} \cdot r_{e}}{2 \cdot f_{z}}
$$


Este ángulo será el de entrada de la herramienta en la pieza en condiciones de fresado ideal, y por tanto, en el corte asimétrico descrito en el capítulo anterior, por eso se ha llamado $\alpha_{a c}$.

Considerando que el corte comienza con una fase de corte asimétrico, realizado sólo por el diente 1, el comienzo del corte simétrico corresponderá al ángulo en el que el diente 2 inicie el corte, cuando el espesor de viruta correspondiente supere el valor mínimo. Para determinar el ángulo en el que ocurre esto se iguala la expresión (4.10) al espesor de viruta crítico:

$$
\begin{aligned}
& t_{c \min }=f_{z} \cdot \sin \phi-2 \cdot \rho \cdot \cos (\alpha-\phi-\lambda)+ \\
& \frac{1}{M} \cdot\left[1-\frac{K_{2}}{K_{1}} \cdot a_{p} \cdot\left(\frac{1}{2}-\frac{\alpha-\phi}{\delta}\right)\right] \cdot\left[\left(F_{x}(1)-F_{x}(2)\right) \cdot \sin \phi+\left(F_{y}(1)-F_{y}(2)\right) \cdot \cos \phi\right]
\end{aligned}
$$

Los componentes $x$ e $y$ de la fuerza sobre el diente 1 se pueden evaluar teniendo en cuenta que en ese punto actuarán las fuerzas de recalcado y las fuerzas de corte asimétrico, antes y después respectivamente del ángulo límite del corte asimétrico.

Las fuerzas de recalcado se podrán deducir a partir de las expresiones (3.18) y (3.19) suprimiendo los términos que multiplican a $f_{\mathrm{z}}$ :

$$
\begin{aligned}
& F_{x}(1)=\frac{D}{2 \cdot \tan \beta} \cdot\left[K_{t e} \cdot \sin \alpha_{a c}-K_{r e} \cdot\left(\cos \alpha_{a c}-1\right)\right] \\
& F_{y}(1)=\frac{D}{2 \cdot \tan \beta} \cdot\left[K_{t e} \cdot\left(\cos \alpha_{a c}-1\right)+K_{r e} \cdot \sin \alpha_{a c}\right]
\end{aligned}
$$

Las fuerzas de corte simétrico se deducen a partir de las expresiones (3.49) y (3.50), estableciendo $\alpha$ y $\alpha_{a c}$ como ángulos límite:

$$
F_{x}(1)=\frac{D}{2 \cdot \tan \beta} \cdot\left[\begin{array}{l}
K_{t e} \cdot\left(\sin \alpha-\sin \alpha_{a c}\right)-K_{r e} \cdot\left(\cos \alpha-\cos \alpha_{a c}\right)+ \\
\frac{f_{z}}{2} \cdot\left[\begin{array}{l}
-K_{t c} \cdot\left(\cos 2 \alpha-\cos 2 \alpha_{a c}\right)+2 K_{r c} \cdot\left(\alpha-\alpha_{a c}\right)- \\
K_{r c} \cdot\left(\sin 2 \alpha-\sin 2 \alpha_{a c}\right)
\end{array}\right]
\end{array}\right]
$$




$$
F_{y}(1)=\frac{D}{2 \cdot \tan \beta} \cdot\left[\begin{array}{l}
K_{t e} \cdot\left(\cos \alpha-\cos \alpha_{a c}\right)+K_{r e} \cdot\left(\sin \alpha-\sin \alpha_{a c}\right)+ \\
\frac{f_{z}}{2} \cdot\left[\begin{array}{l}
-2 K_{t c} \cdot\left(\alpha-\alpha_{a c}\right)+K_{t c} \cdot\left(\sin 2 \alpha-\sin 2 \alpha_{a c}\right)- \\
K_{r c} \cdot\left(\cos 2 \alpha-\cos 2 \alpha_{a c}\right)
\end{array}\right]
\end{array}\right]
$$

Sumando las fuerzas de recalcado y las de corte asimétrico, se obtiene:

$$
\begin{aligned}
& F_{x}(1)=\frac{D}{2 \cdot \tan \beta} \cdot\left[\begin{array}{l}
K_{t e} \cdot \sin \alpha-K_{r e} \cdot(\cos \alpha-1)+ \\
\frac{f_{z}}{2} \cdot\left[\begin{array}{l}
-K_{t c} \cdot\left(\cos 2 \alpha-\cos 2 \alpha_{a c}\right)+2 K_{r c} \cdot\left(\alpha-\alpha_{a c}\right)- \\
K_{r c} \cdot\left(\sin 2 \alpha-\sin 2 \alpha_{a c}\right)
\end{array}\right]
\end{array}\right] \\
& F_{y}(1)=\frac{D}{2 \cdot \tan \beta} \cdot\left[\begin{array}{l}
K_{t e} \cdot(\cos \alpha-1)+K_{r e} \cdot \sin \alpha+ \\
\frac{f_{z}}{2} \cdot\left[\begin{array}{l}
-2 K_{t c} \cdot\left(\alpha-\alpha_{a c}\right)+K_{t c} \cdot\left(\sin 2 \alpha-\sin 2 \alpha_{a c}\right)- \\
K_{r c} \cdot\left(\cos 2 \alpha-\cos 2 \alpha_{a c}\right)
\end{array}\right]
\end{array}\right]
\end{aligned}
$$

Las fuerzas en el diente 2 serán las correspondientes al recalcado y se podrán obtener mediante las expresiones (3.18) y (3.19) considerando $\alpha$ y $\alpha_{e n 0}$ como ángulos límite, de manera similar a como se obtuvieron las expresiones (4.49) y (4.50):

$$
\begin{aligned}
& F_{x}(2)=\frac{D}{2 \cdot \tan \beta} \cdot\left[K_{t e} \cdot\left(\sin \alpha-\sin \alpha_{e n 0}\right)-K_{r e} \cdot\left(\cos \alpha-\cos \alpha_{e n 0}\right)\right] \\
& F_{y}(2)=\frac{D}{2 \cdot \tan \beta} \cdot\left[K_{t e} \cdot\left(\cos \alpha-\cos \alpha_{e n 0}\right)+K_{r e} \cdot\left(\sin \alpha-\sin \alpha_{e n 0}\right)\right]
\end{aligned}
$$

Para cada punto del filo existirá un ángulo de entrada, que se puede determinar mediante la ecuación (4.10) sustituyendo el término $\alpha-\phi$ por su valor en función de la coordenada $z$ considerada en el diente. Se sustituirán también las fuerzas por su valor en función de las expresiones (4.53) y (4.54) cuando sólo haya corte asimétrico. Cuando además la coordenada $z$ es 0 el término $\alpha-\phi$ se anula y se obtiene la siguiente ecuación, que proporciona el ángulo inicial a partir del cual existirá corte simétrico: 


$$
\begin{gathered}
\frac{D}{2 \cdot M \cdot \tan \beta} \cdot\left[\begin{array}{l}
K_{t e} \cdot \sin \alpha-K_{r e} \cdot(\cos \alpha-1)+ \\
\frac{f_{z}}{2} \cdot\left[\begin{array}{l}
-K_{t c} \cdot\left(\cos 2 \alpha-\cos 2 \alpha_{a c}\right)+2 K_{r c} \cdot\left(\alpha-\alpha_{a c}\right)- \\
K_{r c} \cdot\left(\sin 2 \alpha-\sin 2 \alpha_{a c}\right)
\end{array}\right]
\end{array}\right] \cdot \sin \alpha+ \\
\frac{D}{2 \cdot M \cdot \tan \beta} \cdot\left[\begin{array}{l}
K_{t e} \cdot(\cos \alpha-1)+K_{r e} \cdot \sin \alpha+ \\
\left.\frac{f_{z}}{2} \cdot\left[\begin{array}{l}
-2 K_{t c} \cdot\left(\alpha-\alpha_{a c}\right)+K_{t c} \cdot\left(\sin 2 \alpha-\sin 2 \alpha_{a c}\right)- \\
K_{r c} \cdot\left(\cos 2 \alpha-\cos 2 \alpha_{a c}\right)
\end{array}\right]\right) \cdot \cos \alpha=
\end{array}\right] \\
t_{c \min }-f_{z} \cdot \sin \alpha+2 \rho \cdot \cos \lambda
\end{gathered}
$$

Esta ecuación ha de resolverse por métodos numéricos y proporcionará un valor para $\alpha$, que es el límite angular por debajo del cual el segundo filo no puede cortar. Por tanto, antes de que la herramienta alcance ese ángulo sólo existirá corte asimétrico. Téngase en cuenta que la solución se refiere al extremo inferior de la herramienta, que es el punto más adelantado del diente.

Otra posición que se ha de determinar es el ángulo $\alpha_{e n 0}$ en el cual el diente 2 entra en contacto con la pieza. Este ángulo es importante porque marcará el comienzo de las fuerzas de recalcado del diente 2 y se determinará a partir de la expresión (4.57) igualando a 0 el término $t_{c \text { min }}$ :

$$
\begin{aligned}
& \frac{D}{2 \cdot M \cdot \tan \beta} \cdot {\left[\begin{array}{l}
K_{t e} \cdot \sin \alpha_{e n 0}-K_{r e} \cdot\left(\cos \alpha_{e n 0}-1\right)+ \\
\frac{f_{z}}{2} \cdot\left[\begin{array}{l}
-K_{t c} \cdot\left(\cos 2 \alpha_{e n 0}-\cos 2 \alpha_{a c}\right)+ \\
2 K_{r c} \cdot\left(\alpha_{e n 0}-\alpha_{a c}\right)- \\
K_{r c} \cdot\left(\sin 2 \alpha-\sin 2 \alpha_{a c}\right)
\end{array}\right]
\end{array}\right] \cdot \sin \alpha_{e n 0}+} \\
& \frac{D}{2 \cdot M \cdot \tan \beta} \cdot\left[\begin{array}{l}
K_{t e} \cdot\left(\cos \alpha_{e n 0}-1\right)+K_{r e} \cdot \sin \alpha_{e n 0}+ \\
\frac{f_{z}}{2} \cdot\left[\begin{array}{l}
-2 K_{t c} \cdot\left(\alpha_{e n 0}-\alpha_{a c}\right)+ \\
K_{t c} \cdot\left(\sin 2 \alpha_{e n 0}-\sin 2 \alpha_{a c}\right)- \\
K_{r c} \cdot\left(\cos 2 \alpha_{e n 0}-\cos 2 \alpha_{a c}\right)
\end{array}\right]
\end{array}\right] \cdot \cos \alpha_{e n 0}= \\
&-f_{z} \cdot \sin \alpha_{e n 0}+2 \rho \cdot \cos \lambda
\end{aligned}
$$

Para cualquier otro punto del diente, se deberá tener en cuenta la existencia de corte simétrico además del corte simétrico existente en el filo 1 y el recalcado existente en el filo 2:

$$
\vec{F}(1)=\vec{F}_{s c}(1)+\vec{F}_{a c}(1)
$$




$$
\vec{F}(2)=\vec{F}_{s c}(2)+\vec{F}_{e}(2)(4.60)
$$

Las fuerzas correspondientes a corte simétrico pueden ser calculadas mediante el sistema lineal de la expresión (4.21), en el que se deben considerar todas las fuerzas que actúan en cada filo:

$$
\begin{aligned}
& \vec{F}_{s c}(1)=A \cdot\left[\vec{F}_{s c}(1)+\vec{F}_{a c}(1)\right]+\vec{b} \\
& \vec{F}_{s c}(2)=A \cdot\left[\vec{F}_{s c}(2)+\vec{F}_{e}(2)\right]+\vec{b}
\end{aligned}
$$

En estas ecuaciones se puede despejar el vector de fuerzas de corte simétrico:

$$
\begin{aligned}
& \vec{F}_{s c}(1)=(I-A)^{-1} \cdot\left\lfloor A \cdot \vec{F}_{a c}(1)+\vec{b}\right\rfloor \\
& \vec{F}_{s c}(2)=(I-A)^{-1} \cdot\left\lfloor A \cdot \vec{F}_{e}(2)+\vec{b}\right\rfloor
\end{aligned}
$$

Para cada valor de $\alpha$ existirá una matriz $A$ y un vector $b$ que a su vez dependen del ángulo de entrada del diente 2. Como este valor es precisamente el que se desea encontrar, se deberá recurrir a un procedimiento iterativo partiendo de un valor inicial de este ángulo.

De la misma manera, es posible que el diente 2 deje de cortar antes de alcanzar el ángulo máximo teórico $\Phi_{2}$, ya que el espesor de viruta puede verse reducido por la deflexión de herramienta a valores inferiores al valor crítico. Existirá entonces un ángulo de salida del diente 2 que se ha de calcular de forma iterativa, es decir, fijando un ángulo y calculando las fuerzas de corte. Si el espesor de viruta correspondiente es mayor que el mínimo, se aumentará el ángulo hasta que se alcance. A partir de este ángulo de salida habrá sólo corte asimétrico en el diente 1, como en la entrada.

\subsubsection{Resultados experimentales}


Con este modelo se realizó una simulación con los siguientes parámetros: $M=400$ $\mathrm{N} / \mathrm{mm}, D=1 \mathrm{~mm}, a_{e}=0.4 \mathrm{~mm}, a_{p}=0.2 \mathrm{~mm}, f_{\mathrm{z}}=0.01 \mathrm{~mm}, \rho=0.001 \mathrm{~mm}, \lambda=-20^{\circ}, r_{e}=$ $0.001 \mathrm{~mm}, \lambda_{c}=0.33, \beta=30^{\circ}, \delta=13.23^{\circ}$. Estos valores proporcionan los siguientes ángulos de entrada $\alpha_{e n}=0.95^{\circ}$ para $k=1$ y $\alpha_{e n}=5.79^{\circ}$ para $k=2$. Por tanto, habrá un periodo de corte asimétrico entre ambos ángulos de entrada. La fase de entrada se extiende hasta un ángulo de $\alpha=17.79^{\circ}$ porque el ángulo de entrada va variando a medida que el diente avanza. Los resultados de la simulación se muestran en la figura 4.3 .

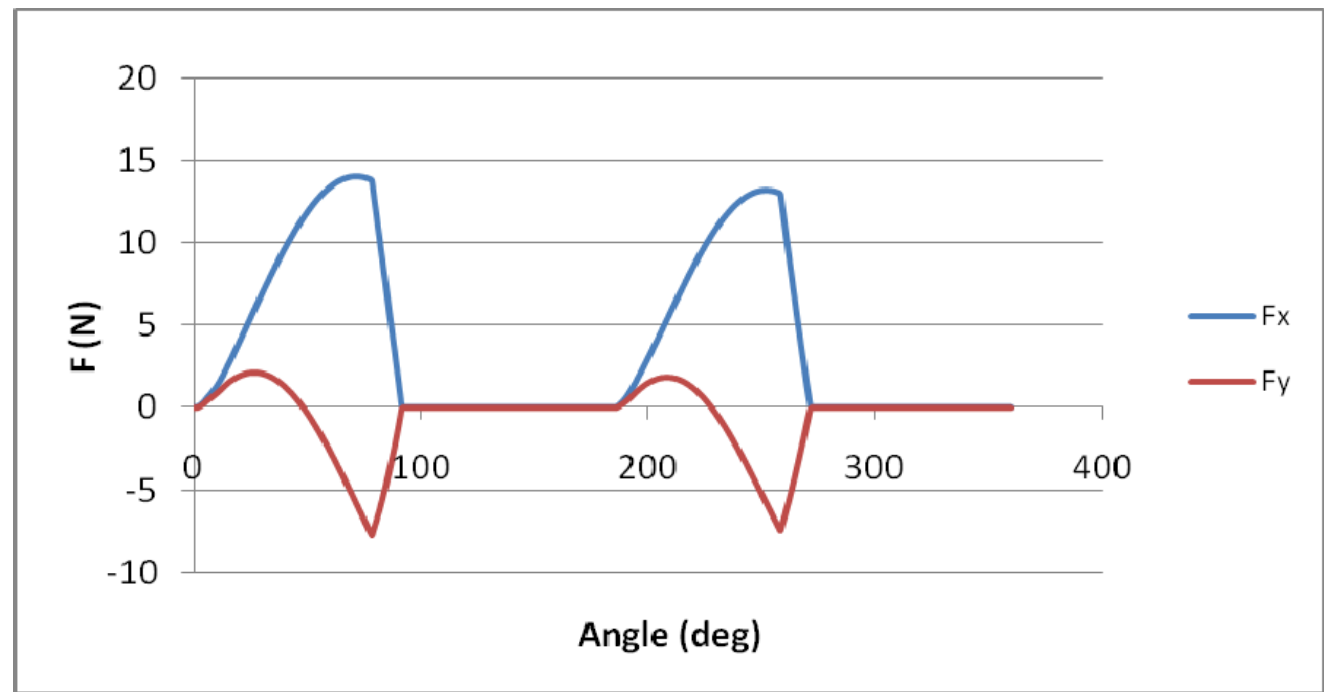

Figura 4.3. Resultados de la simulación del proceso de microfresado en oposición

Para verificar las predicciones del modelo se llevaron a cabo algunos ensayos con la máquina KERN de Microfresado y Microtaladrado en Aluminio 7075. Las condiciones de corte utilizadas fueron las mismas que en la simulación: $D=1 \mathrm{~mm}, a_{e}=0.4 \mathrm{~mm}, a_{p}=$ $0.2 \mathrm{~mm}, f_{\mathrm{z}}=0.01 \mathrm{~mm}, r_{e}=0.001 \mathrm{~mm}, \beta=30^{\circ}, \delta=13.23^{\circ}, \mathrm{n}=13000$ r.p.m. Las fuerzas de corte se midieron con un Dinamómetro KISTLER 9256C2. Los resultados obtenidos se muestran en la figura 7.

Pese a las vibraciones que aparecen en el proceso real se puede observar una gran similitud entre las predicciones y las medidas experimentales. 


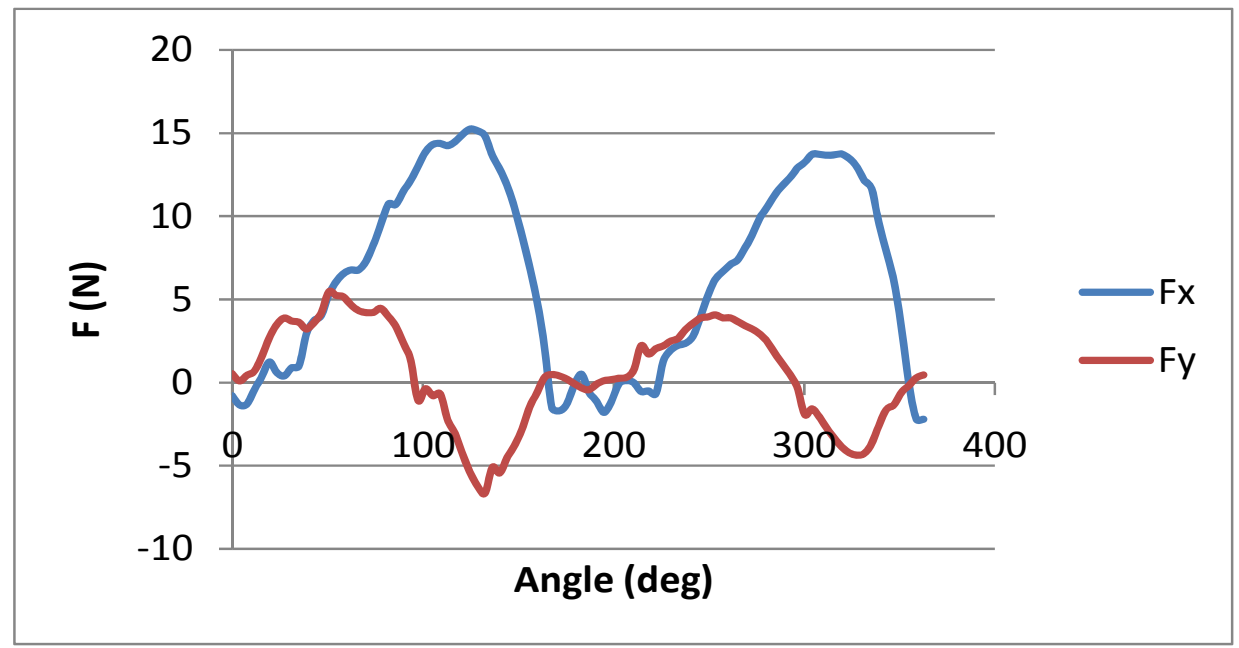

Figura 4.4. Medidas experimentales de la fuerza en microfresado en oposición

Se llevó a cabo otra simulación para una operación de microfresado en oposición con los siguientes parámetros: $M=113 \mathrm{~N} / \mathrm{mm}, D=0.4 \mathrm{~mm}, a_{e}=0.2 \mathrm{~mm}, a_{p}=0.15 \mathrm{~mm}, f_{z}=$ $0.006 \mathrm{~mm}, \rho=0.004 \mathrm{~mm}, \lambda=20^{\circ}, r_{e}=0.001 \mathrm{~mm}, \lambda_{c}=0.3, \beta=30^{\circ}, \delta=24.81^{\circ}$. Estos valores proporcionan los siguientes ángulos de entrada: $\alpha_{e n}=1.43^{\circ}$ para $k=1$ y $\alpha_{e n}=$ $19.65^{\circ}$ para $k=2$. Por tanto, tendrá lugar una fase de corte asimétrico entre esos dos valores. En este caso la fase de entrada se extenderá hasta el ángulo $\alpha=53.86^{\circ}$, ya que el ángulo de entrada irá variando a medida que el diente avanza. En la figura 4.5 se muestran los resultados de la simulación.

En la figura 4.6 se muestran los resultados de la simulación mostrada en la figura 4.5. Las condiciones de corte fueron las siguientes: $D=0.4 \mathrm{~mm}, a_{e}=0.2 \mathrm{~mm}, a_{p}=0.15 \mathrm{~mm}$, $f_{z}=0.006 \mathrm{~mm}, r_{e}=0.001 \mathrm{~mm}, \beta=30^{\circ}, \delta=24.81^{\circ}, \mathrm{n}=18000$ r.p.m. 


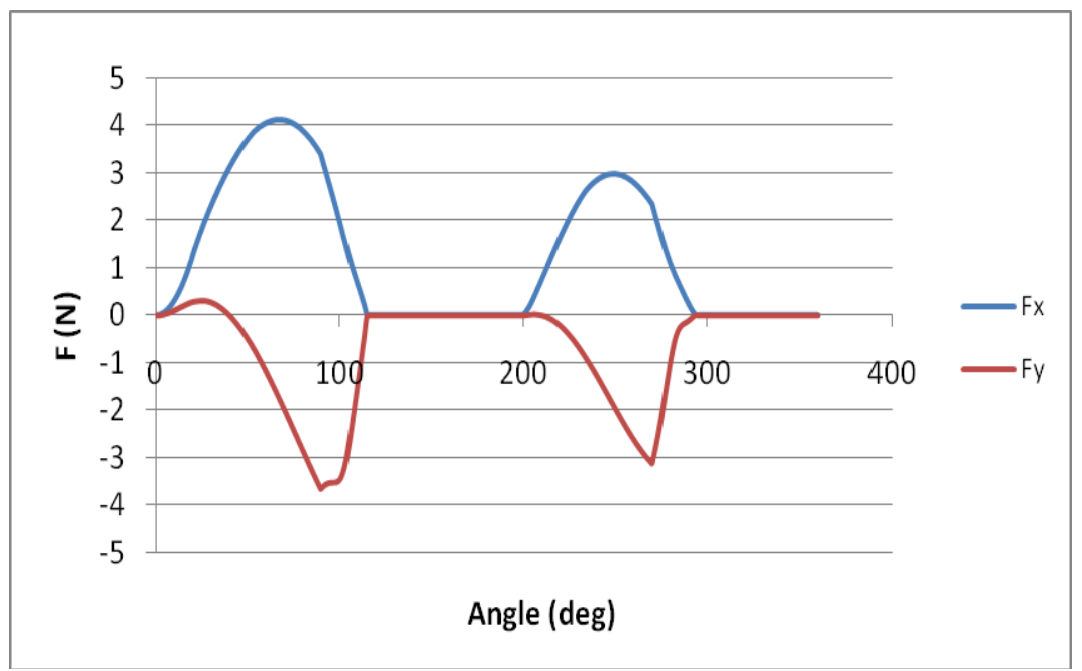

Figura 4.5. Resultados de la simulación del proceso de microfresado en oposición

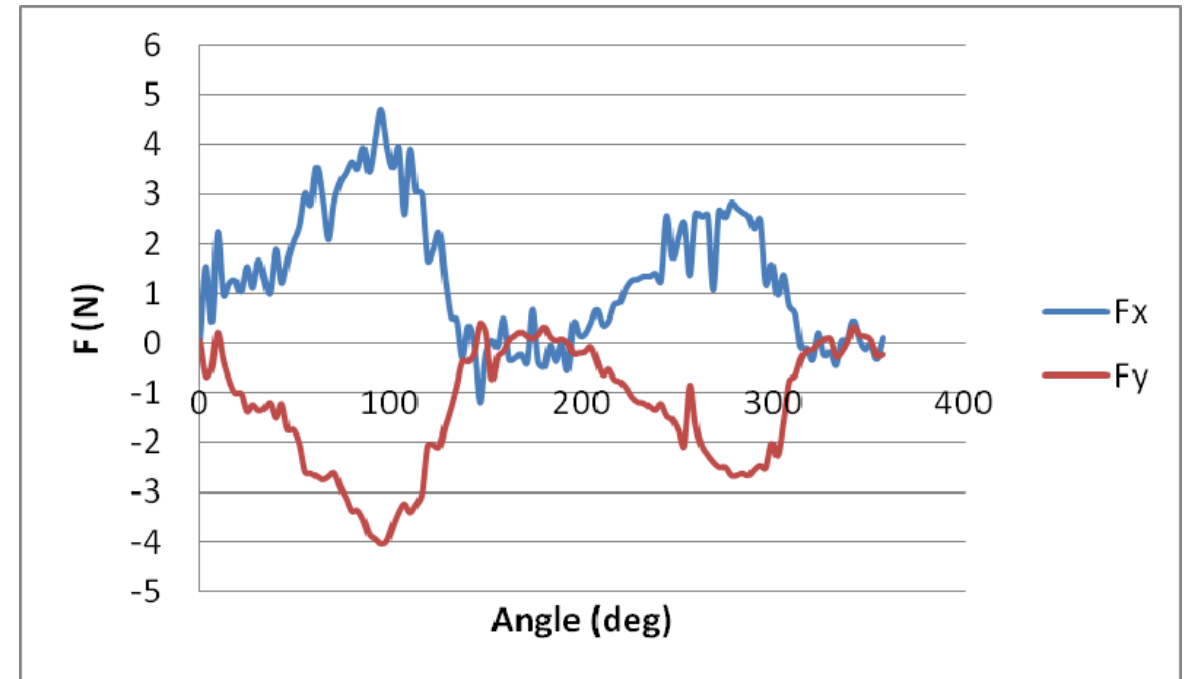

Figura 4.6. Medidas experimentales de la fuerza en microfresado en oposición 


\subsection{Microfresado en concordancia}

\subsubsection{Procedimiento de cálculo del espesor de viruta en condiciones de corte simétrico}

El esquema geométrico de las operaciones de fresado en concordancia es análogo al del fresado en oposición visto en el capítulo anterior. En la figura 4.7 se presenta este esquema, en el que se pueden ver las trayectorias distorsionadas de los dos dientes y el espesor de viruta que cortará el diente 1.

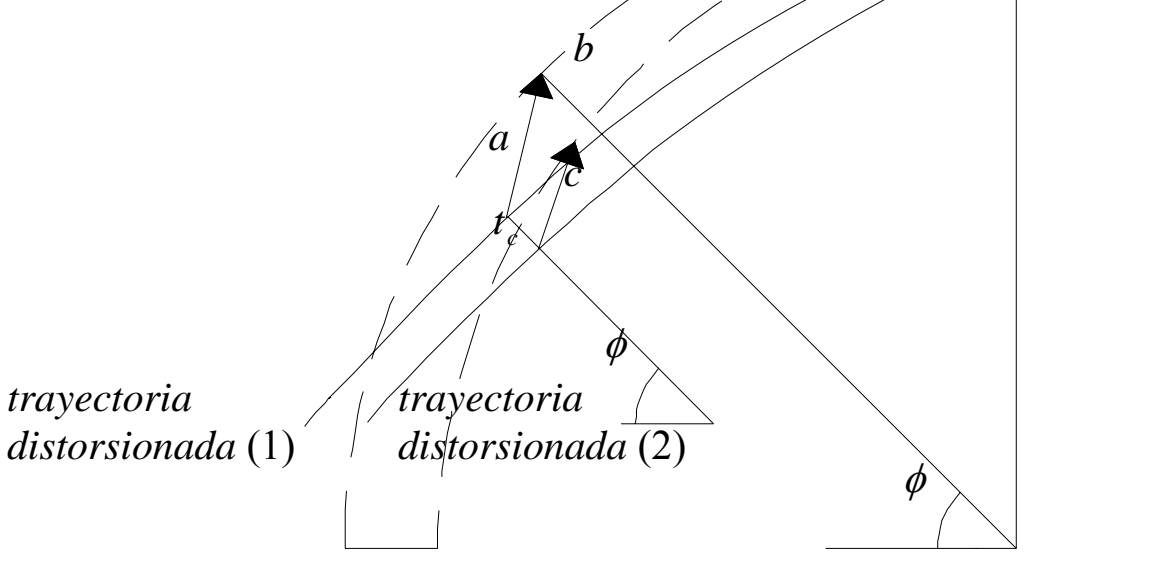

Figura 4.7. Trayectorias distorsionadas de los dientes para fresado en concordancia

En la figura 4.8 se presenta este esquema con más nivel de detalle. 


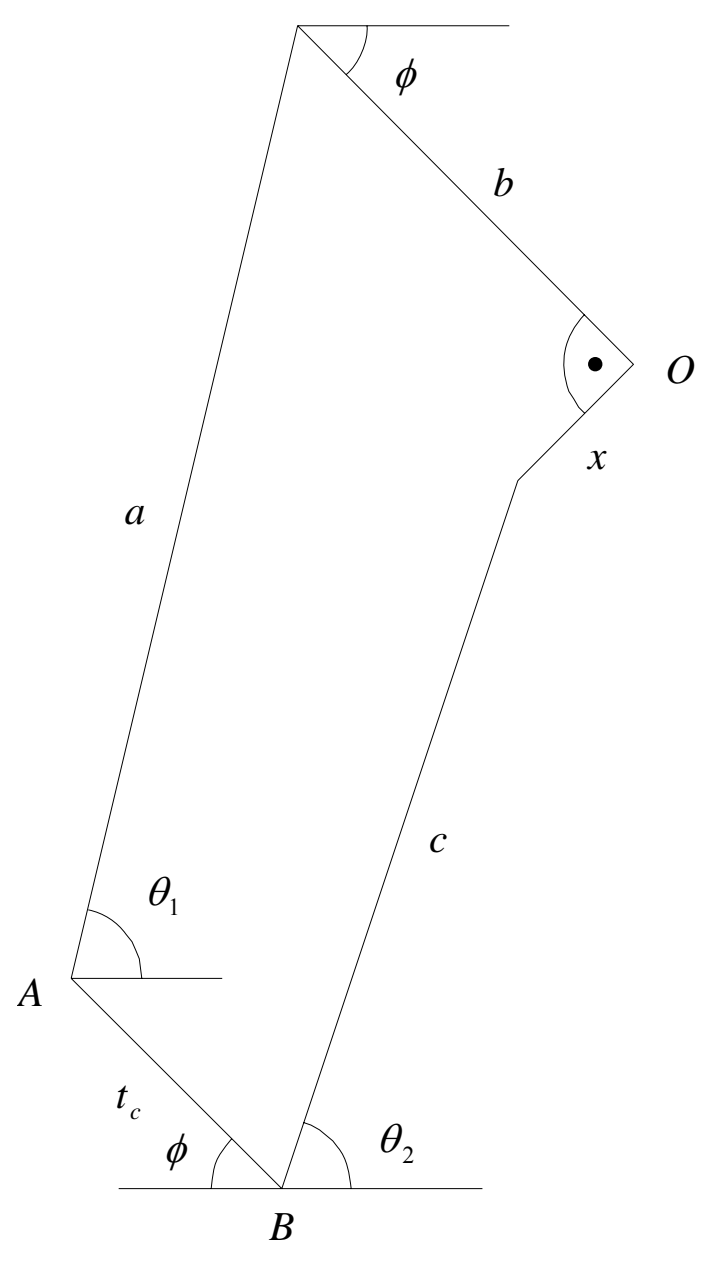

Figura 4.8. Detalle del espesor de viruta para el diente 1 para fresado en concordancia

Hay dos diferencias principales entre fresado en oposición y en concordancia. Por un lado la referencia del ángulo de posición $\phi$ cambia, situándose en la línea horizontal que pasa por el centro de la herramienta, mientras que para el fresado en oposición estaba en la vertical. Por otro lado el esquema es simétrico al del fresado en oposición y, por tanto, el signo de las componentes horizontales de las fuerzas es contrario al del fresado en oposición. En consecuencia, todas las expresiones determinadas para el fresado en oposición se pueden usar para el fresado en concordancia sustituyendo el ángulo $\phi$ por su complementario y cambiando el signo de las componentes horizontales de los vectores $a$ y $c$ : 


$$
\begin{aligned}
& t_{c}(1)=f_{z} \cdot \cos \phi+2 \rho \cos (\alpha-\phi-\lambda)+ \\
& \frac{1}{M} \cdot\left[1-\frac{K_{2}}{K_{1}} \cdot a_{p} \cdot\left(\frac{1}{2}-\frac{\alpha-\phi}{\delta}\right)\right] \cdot\left[\left(F_{x}(1)-F_{x}(2)\right) \cdot \cos \phi+\left(-F_{y}(1)+F_{y}(2)\right) \cdot \sin \phi\right] \\
& t_{c}(2)=f_{z} \cdot \cos \phi-2 \rho \cos (\alpha-\phi-\lambda)+ \\
& \frac{1}{M} \cdot\left[1-\frac{K_{2}}{K_{1}} \cdot a_{p} \cdot\left(\frac{1}{2}-\frac{\alpha-\phi}{\delta}\right)\right] \cdot\left[\left(-F_{x}(1)+F_{x}(2)\right) \cdot \cos \phi+\left(F_{y}(1)-F_{y}(2)\right) \cdot \sin \phi\right]
\end{aligned}
$$

Para calcular las fuerzas de corte será preciso utilizar las expresiones (3.28) y (3.29) generalizadas para los dos dientes:

$$
\begin{gathered}
d F_{x}(k)=\frac{D}{2 \cdot \tan \beta} \cdot\left[\begin{array}{l}
K_{t e} \cdot \sin \phi-K_{r e} \cdot \cos \phi+ \\
\left(K_{t c} \cdot \sin \phi-K_{r c} \cdot \cos \phi\right) \cdot t_{c}(k)
\end{array}\right] \cdot d \phi(4.67) \\
d F_{y}(k)=\frac{D}{2 \cdot \tan \beta} \cdot\left[\begin{array}{l}
K_{t e} \cdot \cos \phi+K_{r e} \cdot \sin \phi+ \\
\left(K_{t c} \cdot \cos \phi+K_{r c} \cdot \sin \phi\right) \cdot t_{c}(k)
\end{array}\right] \cdot d \phi \\
k=1,2
\end{gathered}
$$

En estas expresiones puede sustituirse el espesor de viruta por el valor obtenido en las expresiones (4.65) y (4.66):

$$
d F_{x}(1)=\frac{D}{2 \cdot \tan \beta} \cdot\left[\begin{array}{l}
K_{t e} \cdot \sin \phi-K_{r e} \cdot \cos \phi+ \\
\left(K_{t c} \cdot \sin \phi-K_{r c} \cdot \cos \phi\right) \cdot \\
{\left[\begin{array}{l}
f_{z} \cdot \cos \phi+2 \rho \cos (\alpha-\phi-\lambda)+ \\
\frac{1}{M} \cdot\left[1-\frac{K_{2}}{K_{1}} \cdot a_{p} \cdot\left(\frac{1}{2}-\frac{\alpha-\phi}{\delta}\right)\right] \cdot\left[\begin{array}{l}
\left(F_{x}(1)-F_{x}(2)\right) \cdot \cos \phi+ \\
\left(-F_{y}(1)+F_{y}(2)\right) \cdot \sin \phi
\end{array}\right]
\end{array}\right]}
\end{array}\right] \cdot d \phi
$$




$$
d F_{y}(1)=\frac{D}{2 \cdot \tan \beta} \cdot\left[\begin{array}{l}
K_{t e} \cdot \cos \phi+K_{r e} \cdot \sin \phi+ \\
\left(K_{t c} \cdot \cos \phi+K_{r c} \cdot \sin \phi\right) \cdot \\
{\left[\begin{array}{l}
f_{z} \cdot \cos \phi+2 \rho \cos (\alpha-\phi-\lambda)+ \\
\frac{1}{M} \cdot\left[1-\frac{K_{2}}{K_{1}} \cdot a_{p} \cdot\left(\frac{1}{2}-\frac{\alpha-\phi}{\delta}\right)\right] \cdot\left[\begin{array}{l}
\left(F_{x}(1)-F_{x}(2)\right) \cdot \cos \phi+ \\
\left(-F_{y}(1)+F_{y}(2)\right) \cdot \sin \phi
\end{array}\right]
\end{array}\right]}
\end{array}\right] \cdot d \phi
$$

$d F_{x}(2)=\frac{D}{2 \cdot \tan \beta} \cdot\left[\begin{array}{l}K_{t e} \cdot \sin \phi-K_{r e} \cdot \cos \phi+ \\ \left(K_{t c} \cdot \sin \phi-K_{r c} \cdot \cos \phi\right) \cdot \\ {\left[\begin{array}{l}f_{z} \cdot \cos \phi-2 \rho \cos (\alpha-\phi-\lambda)+ \\ \frac{1}{M} \cdot\left[1-\frac{K_{2}}{K_{1}} \cdot a_{p} \cdot\left(\frac{1}{2}-\frac{\alpha-\phi}{\delta}\right)\right] \cdot\left[\begin{array}{l}\left(-F_{x}(1)+F_{x}(2)\right) \cdot \sin \phi+ \\ \left(F_{y}(1)-F_{y}(2)\right) \cdot \cos \phi\end{array}\right]\end{array}\right]}\end{array}\right] \cdot d \phi$

$$
\left.d F_{y}(2)=\frac{D}{2 \cdot \tan \beta} \cdot\left[\begin{array}{l}
\left(K_{t c} \cdot \cos \phi+K_{r c} \cdot \sin \phi\right) \cdot \\
K_{t} \cdot \cos \phi-2 \rho \cos (\alpha-\phi-\lambda)+ \\
\frac{1}{M} \cdot\left[1-\frac{K_{2}}{K_{1}} \cdot a_{p} \cdot\left(\frac{1}{2}-\frac{\alpha-\phi}{\delta}\right)\right] \cdot\left[\begin{array}{l}
\left.\left(-F_{x}(1)+F_{x}(2)\right) \cdot \sin \phi+\right] \\
\left(F_{y}(1)-F_{y}(2)\right) \cdot \cos \phi
\end{array}\right]
\end{array}\right]\right] \cdot d \phi
$$

Estas expresiones pueden ser integradas, como se hizo en la sección anterior, considerando las fuerzas de corte constantes a lo largo del filo: 


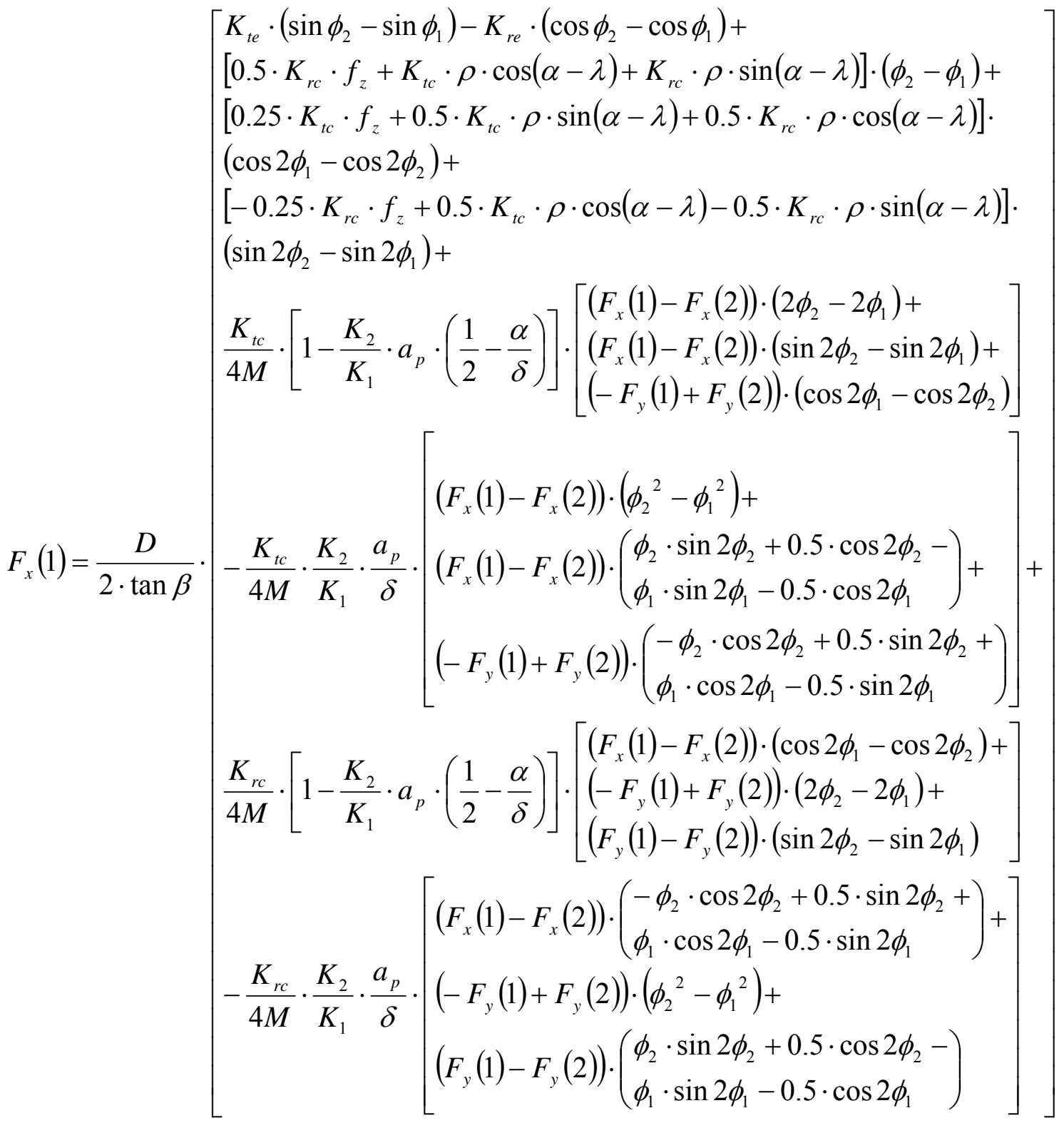




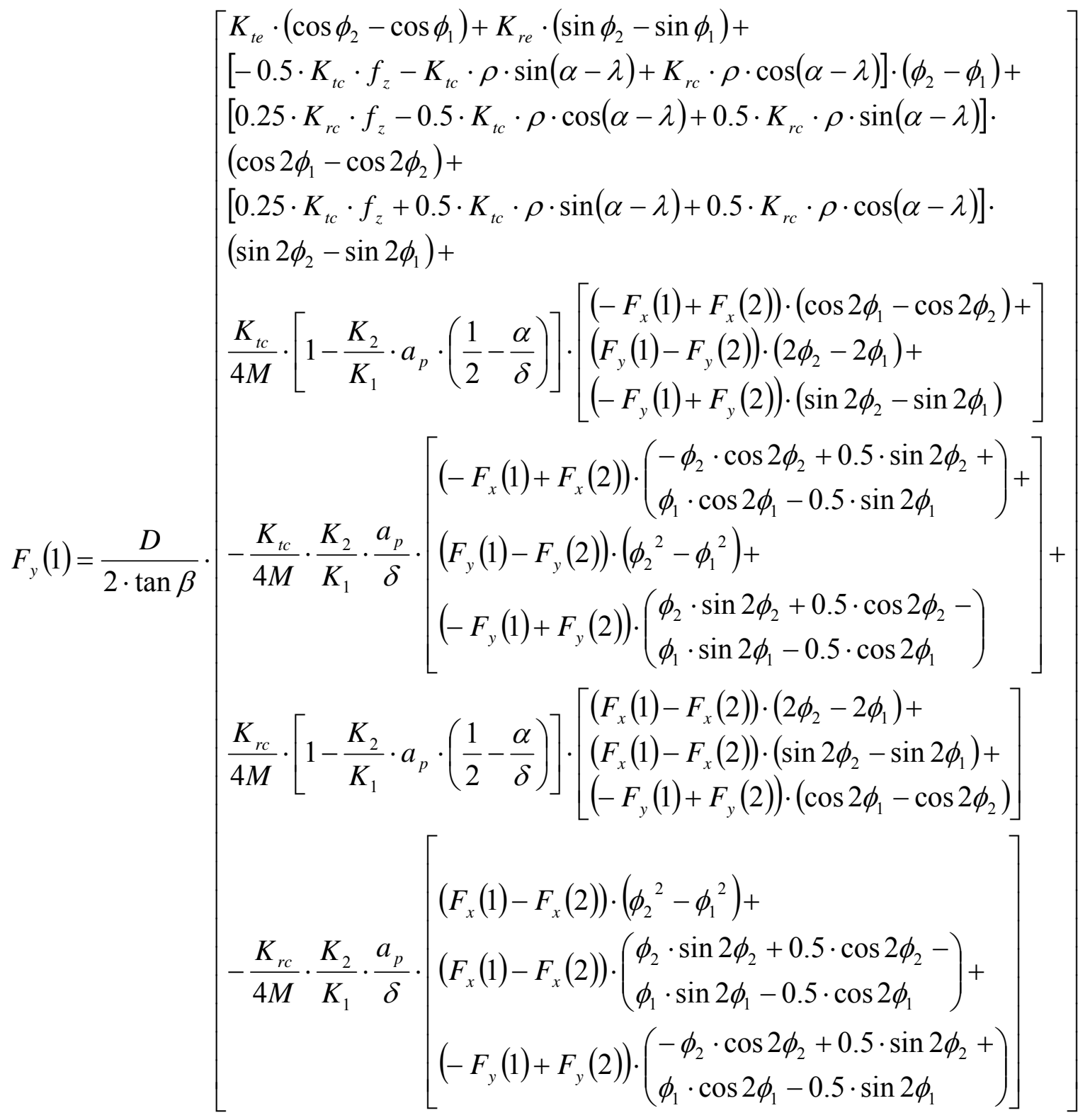




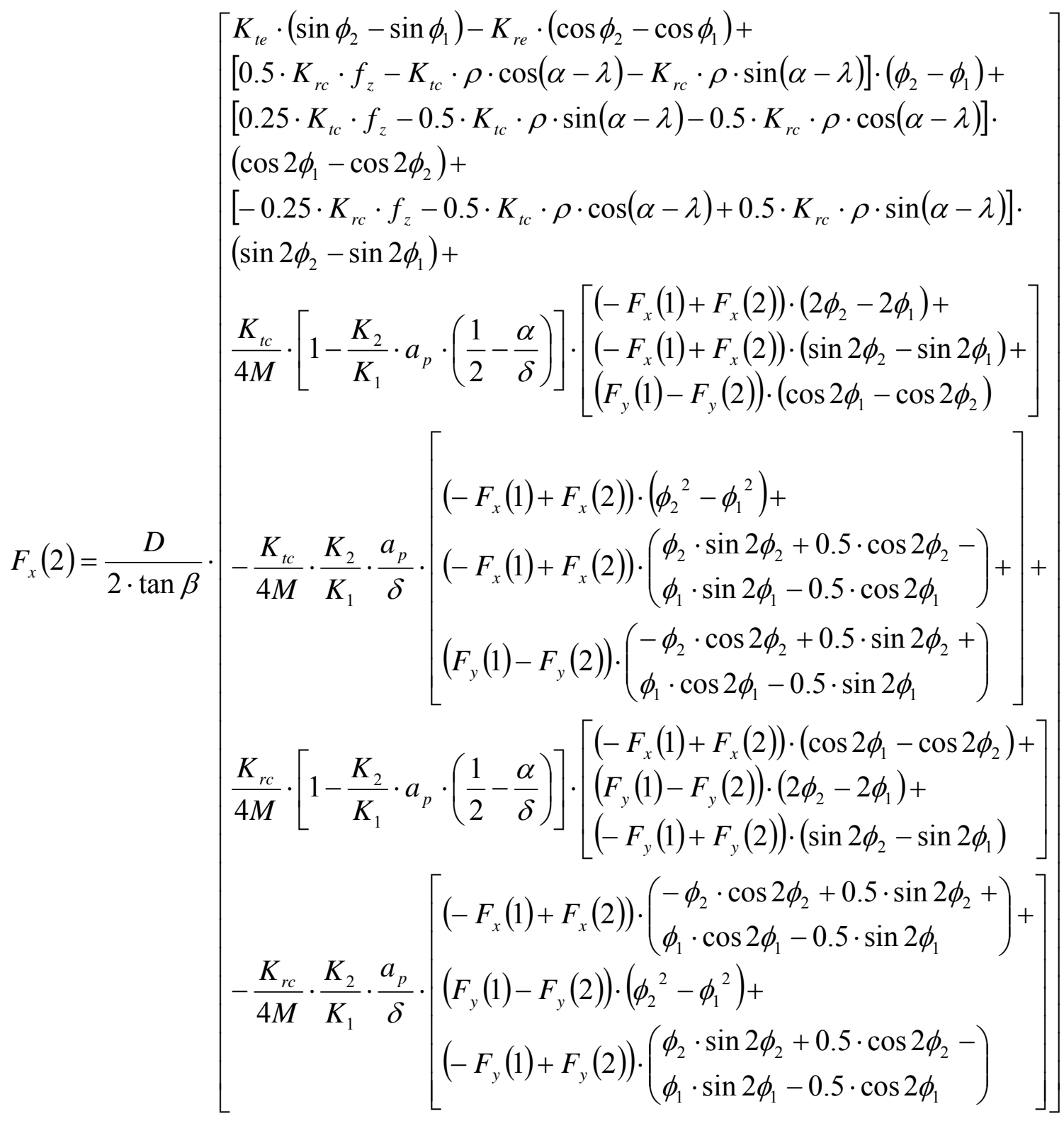




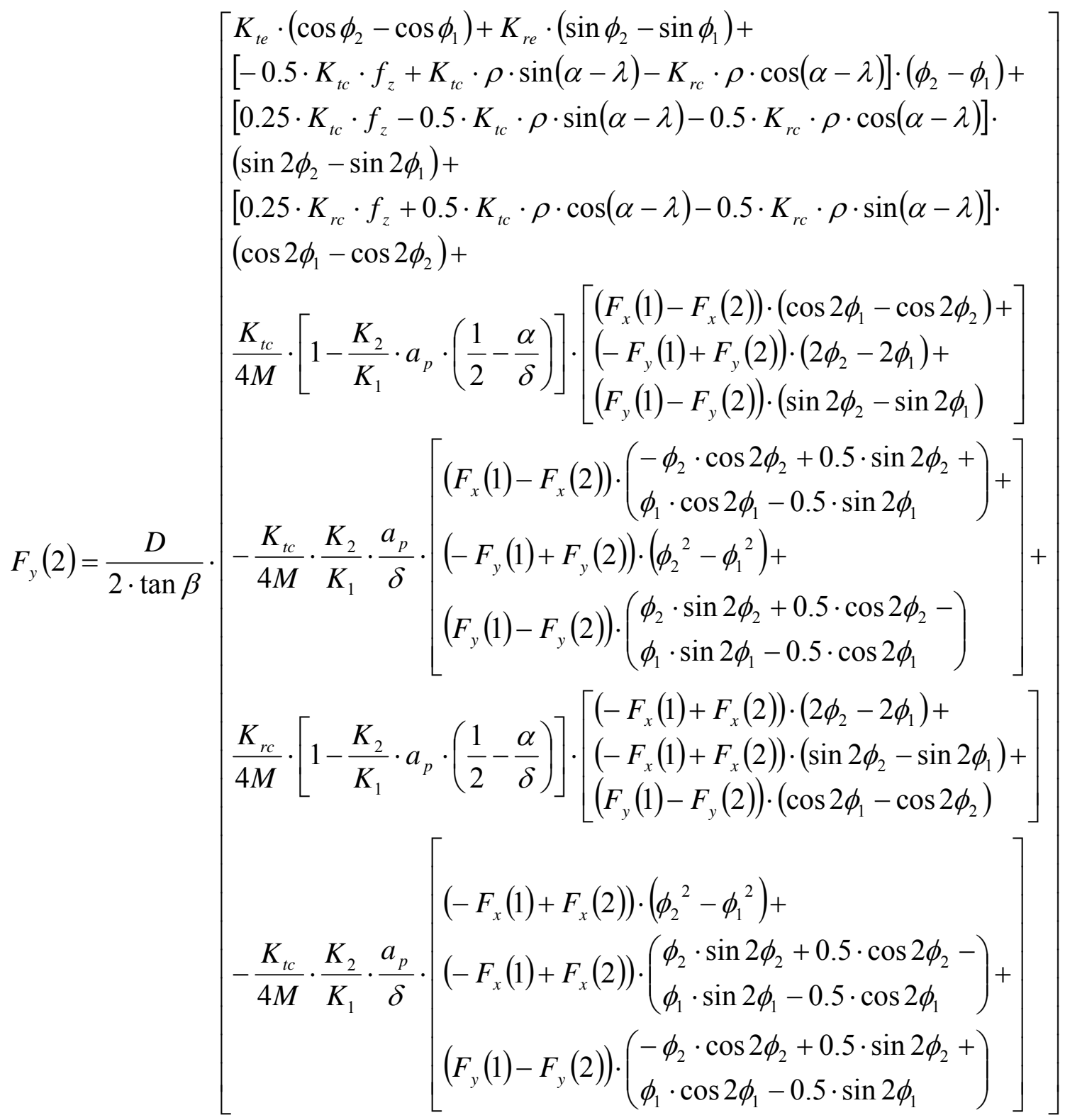

De igual manera que se estableció un sistema de ecuaciones lineales con las fuerzas en la sección anterior, se procederá a asimilar estas expresiones a una ecuación matricial como se hizo en el capítulo anterior en la expresión (4.21):

$$
\vec{F}=A \cdot \vec{F}+\vec{b}
$$


Donde los coeficientes de la matriz A se pueden extraer de las expresiones (4.71)-(4.74), al igual que los componentes $b_{i}$ del vector $\boldsymbol{b}$ :

$$
a_{11}=\frac{D}{2 \cdot \tan \beta} \cdot\left[\begin{array}{l}
\frac{K_{t c}}{4 M} \cdot\left[1-\frac{K_{2}}{K_{1}} \cdot a_{p} \cdot\left(\frac{1}{2}-\frac{\alpha}{\delta}\right)\right] \cdot\left[\left(2 \phi_{2}-2 \phi_{1}\right)+\left(\sin 2 \phi_{2}-\sin 2 \phi_{1}\right)\right] \\
-\frac{K_{t c}}{4 M} \cdot \frac{K_{2}}{K_{1}} \cdot \frac{a_{p}}{\delta} \cdot\left[\left(\phi_{2}^{2}-\phi_{1}^{2}\right)+\left(\begin{array}{l}
\left.\phi_{2} \cdot \sin 2 \phi_{2}+0.5 \cdot \cos 2 \phi_{2}-\right) \\
\phi_{1} \cdot \sin 2 \phi_{1}-0.5 \cdot \cos 2 \phi_{1}
\end{array}\right)\right]+ \\
\frac{K_{r c}}{4 M} \cdot\left[1-\frac{K_{2}}{K_{1}} \cdot a_{p} \cdot\left(\frac{1}{2}-\frac{\alpha}{\delta}\right)\right] \cdot\left(\cos 2 \phi_{1}-\cos 2 \phi_{2}\right) \\
-\frac{K_{r c}}{4 M} \cdot \frac{K_{2}}{K_{1}} \cdot \frac{a_{p}}{\delta} \cdot\left(\begin{array}{l}
-\phi_{2} \cdot \cos 2 \phi_{2}+0.5 \cdot \sin 2 \phi_{2}+ \\
\phi_{1} \cdot \cos 2 \phi_{1}-0.5 \cdot \sin 2 \phi_{1}
\end{array}\right)
\end{array}\right]
$$

$$
a_{12}=\frac{D}{2 \cdot \tan \beta} \cdot\left[\begin{array}{l}
\frac{K_{t c}}{4 M} \cdot\left[1-\frac{K_{2}}{K_{1}} \cdot a_{p} \cdot\left(\frac{1}{2}-\frac{\alpha}{\delta}\right)\right] \cdot\left(\cos 2 \phi_{2}-\cos 2 \phi_{1}\right) \\
-\frac{K_{t c}}{4 M} \cdot \frac{K_{2}}{K_{1}} \cdot \frac{a_{p}}{\delta} \cdot\left(\begin{array}{l}
\left.\phi_{2} \cdot \cos 2 \phi_{2}-0.5 \cdot \sin 2 \phi_{2}-\right) \\
\phi_{1} \cdot \cos 2 \phi_{1}+0.5 \cdot \sin 2 \phi_{1}
\end{array}\right)+ \\
\frac{K_{r c}}{4 M} \cdot\left[1-\frac{K_{2}}{K_{1}} \cdot a_{p} \cdot\left(\frac{1}{2}-\frac{\alpha}{\delta}\right)\right] \cdot\left[\left(2 \phi_{1}-2 \phi_{2}\right)+\left(\sin 2 \phi_{2}-\sin 2 \phi_{1}\right)\right] \\
-\frac{K_{r c}}{4 M} \cdot \frac{K_{2}}{K_{1}} \cdot \frac{a_{p}}{\delta} \cdot\left[\left(\phi_{1}^{2}-\phi_{2}{ }^{2}\right)+\left(\begin{array}{l}
\phi_{2} \cdot \sin 2 \phi_{2}+0.5 \cdot \cos 2 \phi_{2}- \\
\phi_{1} \cdot \sin 2 \phi_{1}-0.5 \cdot \cos 2 \phi_{1}
\end{array}\right)\right]
\end{array}\right]
$$

$$
a_{13}=\frac{D}{2 \cdot \tan \beta} \cdot\left[\begin{array}{l}
\frac{K_{t c}}{4 M} \cdot\left[1-\frac{K_{2}}{K_{1}} \cdot a_{p} \cdot\left(\frac{1}{2}-\frac{\alpha}{\delta}\right)\right] \cdot\left[\left(2 \phi_{1}-2 \phi_{2}\right)+\left(\sin 2 \phi_{1}-\sin 2 \phi_{2}\right)\right] \\
-\frac{K_{t c}}{4 M} \cdot \frac{K_{2}}{K_{1}} \cdot \frac{a_{p}}{\delta} \cdot\left[\left(\phi_{1}^{2}-\phi_{2}^{2}\right)+\left(\begin{array}{l}
\left.-\phi_{2} \cdot \sin 2 \phi_{2}-0.5 \cdot \cos 2 \phi_{2}+\right) \\
\phi_{1} \cdot \sin 2 \phi_{1}+0.5 \cdot \cos 2 \phi_{1}
\end{array}\right)\right]+ \\
\frac{K_{r c}}{4 M} \cdot\left[1-\frac{K_{2}}{K_{1}} \cdot a_{p} \cdot\left(\frac{1}{2}-\frac{\alpha}{\delta}\right)\right] \cdot\left(\cos 2 \phi_{2}-\cos 2 \phi_{1}\right) \\
-\frac{K_{r c}}{4 M} \cdot \frac{K_{2}}{K_{1}} \cdot \frac{a_{p}}{\delta} \cdot\left(\begin{array}{l}
\phi_{2} \cdot \cos 2 \phi_{2}-0.5 \cdot \sin 2 \phi_{2}- \\
\phi_{1} \cdot \cos 2 \phi_{1}+0.5 \cdot \sin 2 \phi_{1}
\end{array}\right)
\end{array}\right]
$$




$$
a_{14}=\frac{D}{2 \cdot \tan \beta} \cdot\left[\begin{array}{l}
\frac{K_{t c}}{4 M} \cdot\left[1-\frac{K_{2}}{K_{1}} \cdot a_{p} \cdot\left(\frac{1}{2}-\frac{\alpha}{\delta}\right)\right] \cdot\left(\cos 2 \phi_{2}-\cos 2 \phi_{1}\right) \\
-\frac{K_{t c}}{4 M} \cdot \frac{K_{2}}{K_{1}} \cdot \frac{a_{p}}{\delta} \cdot\left(\begin{array}{l}
\left.-\phi_{2} \cdot \cos 2 \phi_{2}+0.5 \cdot \sin 2 \phi_{2}+\right) \\
\phi_{1} \cdot \cos 2 \phi_{1}-0.5 \cdot \sin 2 \phi_{1}
\end{array}\right)+ \\
\frac{K_{r c}}{4 M} \cdot\left[1-\frac{K_{2}}{K_{1}} \cdot a_{p} \cdot\left(\frac{1}{2}-\frac{\alpha}{\delta}\right)\right] \cdot\left[\left(2 \phi_{2}-2 \phi_{1}\right)+\left(\sin 2 \phi_{1}-\sin 2 \phi_{2}\right)\right] \\
-\frac{K_{r c}}{4 M} \cdot \frac{K_{2}}{K_{1}} \cdot \frac{a_{p}}{\delta} \cdot\left[\left(\phi_{2}{ }^{2}-\phi_{1}{ }^{2}\right)+\left(\begin{array}{l}
-\phi_{2} \cdot \sin 2 \phi_{2}-0.5 \cdot \cos 2 \phi_{2}+ \\
\phi_{1} \cdot \sin 2 \phi_{1}+0.5 \cdot \cos 2 \phi_{1}
\end{array}\right)\right.
\end{array}\right]
$$

$$
a_{21}=\frac{D}{2 \cdot \tan \beta} \cdot\left[\begin{array}{l}
\frac{K_{t c}}{4 M} \cdot\left[1-\frac{K_{2}}{K_{1}} \cdot a_{p} \cdot\left(\frac{1}{2}-\frac{\alpha}{\delta}\right)\right] \cdot\left(\cos 2 \phi_{2}-\cos 2 \phi_{1}\right)+ \\
\frac{K_{t c}}{4 M} \cdot\left(1-\frac{K_{2}}{K_{1}} \cdot \frac{a_{p}}{\delta}\right) \cdot\left(\begin{array}{l}
\phi_{2} \cdot \cos 2 \phi_{2}-0.5 \cdot \sin 2 \phi_{2}- \\
\phi_{1} \cdot \cos 2 \phi_{1}+0.5 \cdot \sin 2 \phi_{1}
\end{array}\right)+ \\
\frac{K_{r c}}{4 M} \cdot\left[1-\frac{K_{2}}{K_{1}} \cdot a_{p} \cdot\left(\frac{1}{2}-\frac{\alpha}{\delta}\right)\right] \cdot\left[\left(2 \phi_{2}-2 \phi_{1}\right)+\left(\sin 2 \phi_{2}-\sin 2 \phi_{1}\right)\right]+ \\
\frac{K_{r c}}{4 M} \cdot\left(1-\frac{K_{2}}{K_{1}} \cdot \frac{a_{p}}{\delta}\right) \cdot\left[\left(\phi_{2}^{2}-\phi_{1}^{2}\right)+\left(\begin{array}{l}
\phi_{2} \cdot \sin 2 \phi_{2}+0.5 \cdot \cos 2 \phi_{2}- \\
\phi_{1} \cdot \sin 2 \phi_{1}-0.5 \cdot \cos 2 \phi_{1}
\end{array}\right)\right]
\end{array}\right]
$$

$$
a_{22}=\frac{D}{2 \cdot \tan \beta} \cdot\left[\begin{array}{l}
\frac{K_{t c}}{4 M} \cdot\left[1-\frac{K_{2}}{K_{1}} \cdot a_{p} \cdot\left(\frac{1}{2}-\frac{\alpha}{\delta}\right)\right] \cdot\left[\left(2 \phi_{2}-2 \phi_{1}\right)+\left(\sin 2 \phi_{1}-\sin 2 \phi_{2}\right)\right] \\
-\frac{K_{t c}}{4 M} \cdot \frac{K_{2}}{K_{1}} \cdot \frac{a_{p}}{\delta} \cdot\left[\left(\phi_{2}^{2}-\phi_{1}^{2}\right)+\left(\begin{array}{l}
\left.-\phi_{2} \cdot \sin 2 \phi_{2}-0.5 \cdot \cos 2 \phi_{2}+\right) \\
\phi_{1} \cdot \sin 2 \phi_{1}+0.5 \cdot \cos 2 \phi_{1}
\end{array}\right)\right]+ \\
\frac{K_{r c}}{4 M} \cdot\left[1-\frac{K_{2}}{K_{1}} \cdot a_{p} \cdot\left(\frac{1}{2}-\frac{\alpha}{\delta}\right)\right] \cdot\left(\cos 2 \phi_{2}-\cos 2 \phi_{1}\right) \\
-\frac{K_{r c}}{4 M} \cdot \frac{K_{2}}{K_{1}} \cdot \frac{a_{p}}{\delta} \cdot\left(\begin{array}{l}
\phi_{2} \cdot \cos 2 \phi_{2}-0.5 \cdot \sin 2 \phi_{2}- \\
\phi_{1} \cdot \cos 2 \phi_{1}+0.5 \cdot \sin 2 \phi_{1}
\end{array}\right)
\end{array}\right]
$$




$$
a_{23}=\frac{D}{2 \cdot \tan \beta} \cdot\left[\begin{array}{l}
\frac{K_{t c}}{4 M} \cdot\left[1-\frac{K_{2}}{K_{1}} \cdot a_{p} \cdot\left(\frac{1}{2}-\frac{\alpha}{\delta}\right)\right] \cdot\left(\cos 2 \phi_{1}-\cos 2 \phi_{2}\right) \\
-\frac{K_{t c}}{4 M} \cdot \frac{K_{2}}{K_{1}} \cdot \frac{a_{p}}{\delta} \cdot\left(\begin{array}{l}
-\phi_{2} \cdot \cos 2 \phi_{2}+0.5 \cdot \sin 2 \phi_{2}+ \\
\phi_{1} \cdot \cos 2 \phi_{1}-0.5 \cdot \sin 2 \phi_{1}
\end{array}\right)+ \\
\frac{K_{r c}}{4 M} \cdot\left[1-\frac{K_{2}}{K_{1}} \cdot a_{p} \cdot\left(\frac{1}{2}-\frac{\alpha}{\delta}\right)\right] \cdot\left[\left(2 \phi_{1}-2 \phi_{2}\right)+\left(\sin 2 \phi_{1}-\sin 2 \phi_{2}\right)\right]
\end{array}\right]
$$




$$
a_{32}=\frac{D}{2 \cdot \tan \beta} \cdot\left[\begin{array}{l}
\frac{K_{t c}}{4 M} \cdot\left[1-\frac{K_{2}}{K_{1}} \cdot a_{p} \cdot\left(\frac{1}{2}-\frac{\alpha}{\delta}\right)\right] \cdot\left(\cos 2 \phi_{1}-\cos 2 \phi_{2}\right) \\
-\frac{K_{t c}}{4 M} \cdot \frac{K_{2}}{K_{1}} \cdot \frac{a_{p}}{\delta} \cdot\left(\begin{array}{l}
\left.-\phi_{2} \cdot \cos 2 \phi_{2}+0.5 \cdot \sin 2 \phi_{2}+\right) \\
\phi_{1} \cdot \cos 2 \phi_{1}-0.5 \cdot \sin 2 \phi_{1}
\end{array}\right)+ \\
\frac{K_{r c}}{4 M} \cdot\left[1-\frac{K_{2}}{K_{1}} \cdot a_{p} \cdot\left(\frac{1}{2}-\frac{\alpha}{\delta}\right)\right] \cdot\left[\left(2 \phi_{2}-2 \phi_{1}\right)+\left(\sin 2 \phi_{1}-\sin 2 \phi_{2}\right)\right] \\
-\frac{K_{r c}}{4 M} \cdot \frac{K_{2}}{K_{1}} \cdot \frac{a_{p}}{\delta} \cdot\left[\left(\phi_{2}{ }^{2}-\phi_{1}{ }^{2}\right)+\left(\begin{array}{l}
-\phi_{2} \cdot \sin 2 \phi_{2}-0.5 \cdot \cos 2 \phi_{2}+ \\
\phi_{1} \cdot \sin 2 \phi_{1}+0.5 \cdot \cos 2 \phi_{1}
\end{array}\right)\right.
\end{array}\right]
$$

$$
a_{33}=\frac{D}{2 \cdot \tan \beta} \cdot\left[\begin{array}{l}
\frac{K_{t c}}{4 M} \cdot\left[1-\frac{K_{2}}{K_{1}} \cdot a_{p} \cdot\left(\frac{1}{2}-\frac{\alpha}{\delta}\right)\right] \cdot\left[\left(2 \phi_{2}-2 \phi_{1}\right)+\left(\sin 2 \phi_{2}-\sin 2 \phi_{1}\right)\right] \\
-\frac{K_{t c}}{4 M} \cdot \frac{K_{2}}{K_{1}} \cdot \frac{a_{p}}{\delta} \cdot\left[\left(\phi_{2}^{2}-\phi_{1}^{2}\right)+\left(\begin{array}{l}
\left.\phi_{2} \cdot \sin 2 \phi_{2}+0.5 \cdot \cos 2 \phi_{2}-\right) \\
\phi_{1} \cdot \sin 2 \phi_{1}-0.5 \cdot \cos 2 \phi_{1}
\end{array}\right)\right]+ \\
\frac{K_{r c}}{4 M} \cdot\left[1-\frac{K_{2}}{K_{1}} \cdot a_{p} \cdot\left(\frac{1}{2}-\frac{\alpha}{\delta}\right)\right] \cdot\left(\cos 2 \phi_{1}-\cos 2 \phi_{2}\right) \\
-\frac{K_{r c}}{4 M} \cdot \frac{K_{2}}{K_{1}} \cdot \frac{a_{p}}{\delta} \cdot\left(\begin{array}{l}
-\phi_{2} \cdot \cos 2 \phi_{2}+0.5 \cdot \sin 2 \phi_{2}+ \\
\phi_{1} \cdot \cos 2 \phi_{1}-0.5 \cdot \sin 2 \phi_{1}
\end{array}\right)
\end{array}\right]
$$

$$
a_{34}=\frac{D}{2 \cdot \tan \beta} \cdot\left[\begin{array}{l}
\frac{K_{t c}}{4 M} \cdot\left[1-\frac{K_{2}}{K_{1}} \cdot a_{p} \cdot\left(\frac{1}{2}-\frac{\alpha}{\delta}\right)\right] \cdot\left(\cos 2 \phi_{2}-\cos 2 \phi_{1}\right) \\
-\frac{K_{t c}}{4 M} \cdot \frac{K_{2}}{K_{1}} \cdot \frac{a_{p}}{\delta} \cdot\left(\begin{array}{l}
\phi_{2} \cdot \cos 2 \phi_{2}-0.5 \cdot \sin 2 \phi_{2}- \\
\phi_{1} \cdot \cos 2 \phi_{1}+0.5 \cdot \sin 2 \phi_{1}
\end{array}\right)+ \\
\frac{K_{r c}}{4 M} \cdot\left[1-\frac{K_{2}}{K_{1}} \cdot a_{p} \cdot\left(\frac{1}{2}-\frac{\alpha}{\delta}\right)\right] \cdot\left[\left(2 \phi_{1}-2 \phi_{2}\right)+\left(\sin 2 \phi_{2}-\sin 2 \phi_{1}\right)\right] \\
-\frac{K_{r c}}{4 M} \cdot \frac{K_{2}}{K_{1}} \cdot \frac{a_{p}}{\delta} \cdot\left[\left(\phi_{1}^{2}-\phi_{2}^{2}\right)+\left(\begin{array}{l}
\left.\phi_{2} \cdot \sin 2 \phi_{2}+0.5 \cdot \cos 2 \phi_{2}-\right) \\
\phi_{1} \cdot \sin 2 \phi_{1}-0.5 \cdot \cos 2 \phi_{1}
\end{array}\right)\right.
\end{array}\right]
$$




$$
\begin{aligned}
& a_{41}=\frac{D}{2 \cdot \tan \beta} \cdot\left[\begin{array}{l}
\frac{K_{t c}}{4 M} \cdot\left[1-\frac{K_{2}}{K_{1}} \cdot a_{p} \cdot\left(\frac{1}{2}-\frac{\alpha}{\delta}\right)\right] \cdot\left(\cos 2 \phi_{1}-\cos 2 \phi_{2}\right) \\
-\frac{K_{t c}}{4 M} \cdot \frac{K_{2}}{K_{1}} \cdot \frac{a_{p}}{\delta} \cdot\left(\begin{array}{l}
\left.-\phi_{2} \cdot \cos 2 \phi_{2}+0.5 \cdot \sin 2 \phi_{2}+\right) \\
\phi_{1} \cdot \cos 2 \phi_{1}-0.5 \cdot \sin 2 \phi_{1}
\end{array}\right)+ \\
\frac{K_{r c}}{4 M} \cdot\left[1-\frac{K_{2}}{K_{1}} \cdot a_{p} \cdot\left(\frac{1}{2}-\frac{\alpha}{\delta}\right)\right] \cdot\left[\left(2 \phi_{1}-2 \phi_{2}\right)+\left(\sin 2 \phi_{1}-\sin 2 \phi_{2}\right)\right] \\
-\frac{K_{r c}}{4 M} \cdot \frac{K_{2}}{K_{1}} \cdot \frac{a_{p}}{\delta} \cdot\left[\left(\phi_{1}^{2}-\phi_{2}^{2}\right)+\left(\begin{array}{l}
\left.-\phi_{2} \cdot \sin 2 \phi_{2}-0.5 \cdot \cos 2 \phi_{2}+\right) \\
\phi_{1} \cdot \sin 2 \phi_{1}+0.5 \cdot \cos 2 \phi_{1}
\end{array}\right)\right]
\end{array}\right] \\
& a_{42}=\frac{D}{2 \cdot \tan \beta} \cdot\left[\begin{array}{l}
\frac{K_{t c}}{4 M} \cdot\left[1-\frac{K_{2}}{K_{1}} \cdot a_{p} \cdot\left(\frac{1}{2}-\frac{\alpha}{\delta}\right)\right] \cdot\left[\left(2 \phi_{1}-2 \phi_{2}\right)+\left(\sin 2 \phi_{2}-\sin 2 \phi_{1}\right)\right] \\
-\frac{K_{t c}}{4 M} \cdot \frac{K_{2}}{K_{1}} \cdot \frac{a_{p}}{\delta} \cdot\left[\left(\phi_{1}^{2}-\phi_{2}{ }^{2}\right)+\left(\begin{array}{l}
\left.\phi_{2} \cdot \sin 2 \phi_{2}+0.5 \cdot \cos 2 \phi_{2}-\right) \\
\phi_{1} \cdot \sin 2 \phi_{1}-0.5 \cdot \cos 2 \phi_{1}
\end{array}\right)\right]+ \\
\frac{K_{r c}}{4 M} \cdot\left[1-\frac{K_{2}}{K_{1}} \cdot a_{p} \cdot\left(\frac{1}{2}-\frac{\alpha}{\delta}\right)\right] \cdot\left(\cos 2 \phi_{1}-\cos 2 \phi_{2}\right) \\
-\frac{K_{r c}}{4 M} \cdot \frac{K_{2}}{K_{1}} \cdot \frac{a_{p}}{\delta} \cdot\left(\begin{array}{l}
-\phi_{2} \cdot \cos 2 \phi_{2}+0.5 \cdot \sin 2 \phi_{2}+ \\
\phi_{1} \cdot \cos 2 \phi_{1}-0.5 \cdot \sin 2 \phi_{1}
\end{array}\right)
\end{array}\right] \\
& a_{43}=\frac{D}{2 \cdot \tan \beta} \cdot\left[\begin{array}{l}
\frac{K_{t c}}{4 M} \cdot\left[1-\frac{K_{2}}{K_{1}} \cdot a_{p} \cdot\left(\frac{1}{2}-\frac{\alpha}{\delta}\right)\right] \cdot\left(\cos 2 \phi_{2}-\cos 2 \phi_{1}\right) \\
-\frac{K_{t c}}{4 M} \cdot \frac{K_{2}}{K_{1}} \cdot \frac{a_{p}}{\delta} \cdot\left(\begin{array}{l}
\left.\phi_{2} \cdot \cos 2 \phi_{2}-0.5 \cdot \sin 2 \phi_{2}-\right) \\
\phi_{1} \cdot \cos 2 \phi_{1}+0.5 \cdot \sin 2 \phi_{1}
\end{array}\right)+ \\
\frac{K_{r c}}{4 M} \cdot\left[1-\frac{K_{2}}{K_{1}} \cdot a_{p} \cdot\left(\frac{1}{2}-\frac{\alpha}{\delta}\right)\right] \cdot\left[\left(2 \phi_{2}-2 \phi_{1}\right)+\left(\sin 2 \phi_{2}-\sin 2 \phi_{1}\right)\right] \\
-\frac{K_{r c}}{4 M} \cdot \frac{K_{2}}{K_{1}} \cdot \frac{a_{p}}{\delta} \cdot\left[\left(\phi_{2}^{2}-\phi_{1}^{2}\right)+\left(\begin{array}{l}
\left.\phi_{2} \cdot \sin 2 \phi_{2}+0.5 \cdot \cos 2 \phi_{2}-\right) \\
\phi_{1} \cdot \sin 2 \phi_{1}-0.5 \cdot \cos 2 \phi_{1}
\end{array}\right)\right]
\end{array}\right]
\end{aligned}
$$




$$
\begin{aligned}
& a_{44}=\frac{D}{2 \cdot \tan \beta} \cdot\left[\begin{array}{l}
\frac{K_{t c}}{4 M} \cdot\left[1-\frac{K_{2}}{K_{1}} \cdot a_{p} \cdot\left(\frac{1}{2}-\frac{\alpha}{\delta}\right)\right] \cdot\left[\left(2 \phi_{2}-2 \phi_{1}\right)+\left(\sin 2 \phi_{1}-\sin 2 \phi_{2}\right)\right] \\
-\frac{K_{t c}}{4 M} \cdot \frac{K_{2}}{K_{1}} \cdot \frac{a_{p}}{\delta} \cdot\left[\left(\phi_{2}^{2}-\phi_{1}^{2}\right)+\left(\begin{array}{l}
-\phi_{2} \cdot \sin 2 \phi_{2}-0.5 \cdot \cos 2 \phi_{2}+ \\
\phi_{1} \cdot \sin 2 \phi_{1}+0.5 \cdot \cos 2 \phi_{1}
\end{array}\right)\right]+ \\
\frac{K_{r c}}{4 M} \cdot\left[1-\frac{K_{2}}{K_{1}} \cdot a_{p} \cdot\left(\frac{1}{2}-\frac{\alpha}{\delta}\right)\right] \cdot\left(\cos 2 \phi_{2}-\cos 2 \phi_{1}\right) \\
-\frac{K_{r c}}{4 M} \cdot \frac{K_{2}}{K_{1}} \cdot \frac{a_{p}}{\delta} \cdot\left(\begin{array}{l}
\phi_{2} \cdot \cos 2 \phi_{2}-0.5 \cdot \sin 2 \phi_{2}- \\
\phi_{1} \cdot \cos 2 \phi_{1}+0.5 \cdot \sin 2 \phi_{1}
\end{array}\right)
\end{array}\right] \\
& b_{1}=\frac{D}{8 \cdot \tan \beta} \cdot\left[\begin{array}{l}
-4 \cdot K_{t e} \cdot\left(\cos \phi_{2}-\cos \phi_{1}\right)-4 \cdot K_{r e} \cdot\left(\sin \phi_{2}-\sin \phi_{1}\right)+ \\
{\left[\begin{array}{l}
-2 \cdot K_{r c} \cdot f_{z}+4 \cdot K_{t c} \cdot \rho \cdot \sin (\alpha-\lambda)- \\
4 \cdot K_{r c} \cdot \rho \cdot \cos (\alpha-\lambda)
\end{array}\right] \cdot\left(\phi_{2}-\phi_{1}\right)+} \\
{\left[\begin{array}{l}
K_{t c} \cdot f_{z}-2 \cdot K_{r c} \cdot \rho \cdot \sin (\alpha-\lambda)+ \\
2 \cdot K_{t c} \cdot \rho \cdot \cos (\alpha-\lambda)
\end{array}\right] \cdot\left(\cos 2 \phi_{1}-\cos 2 \phi_{2}\right)+} \\
{\left[\begin{array}{l}
-K_{r c} \cdot f_{z}-2 \cdot K_{t c} \cdot \rho \cdot \sin (\alpha-\lambda)- \\
2 \cdot K_{r c} \cdot \rho \cdot \cos (\alpha-\lambda)
\end{array}\right] \cdot\left(\sin 2 \phi_{2}-\sin 2 \phi_{1}\right)}
\end{array}\right] \\
& b_{2}=\frac{D}{8 \cdot \tan \beta} \cdot\left[\begin{array}{l}
4 \cdot K_{t e} \cdot\left(\sin \phi_{2}-\sin \phi_{1}\right)-4 \cdot K_{r e} \cdot\left(\cos \phi_{2}-\cos \phi_{1}\right)+ \\
{\left[\begin{array}{l}
2 \cdot K_{t c} \cdot f_{z}+4 \cdot K_{r c} \cdot \rho \cdot \sin (\alpha-\lambda)+ \\
4 \cdot K_{t c} \cdot \rho \cdot \cos (\alpha-\lambda)
\end{array}\right] \cdot\left(\phi_{2}-\phi_{1}\right)+} \\
{\left[\begin{array}{l}
K_{r c} \cdot f_{z}+2 \cdot K_{t c} \cdot \rho \cdot \sin (\alpha-\lambda)+ \\
2 \cdot K_{r c} \cdot \rho \cdot \cos (\alpha-\lambda)
\end{array}\right] \cdot\left(\cos 2 \phi_{1}-\cos 2 \phi_{2}\right)+} \\
{\left[\begin{array}{l}
K_{t c} \cdot f_{z}-2 \cdot K_{r c} \cdot \rho \cdot \sin (\alpha-\lambda)+ \\
2 \cdot K_{t c} \cdot \rho \cdot \cos (\alpha-\lambda)
\end{array}\right] \cdot\left(\sin 2 \phi_{2}-\sin 2 \phi_{1}\right)}
\end{array}\right]
\end{aligned}
$$




$$
b_{4}=\frac{D}{8 \cdot \tan \beta} \cdot\left[\begin{array}{l}
4 \cdot K_{t e} \cdot\left(\sin \phi_{2}-\sin \phi_{1}\right)-4 \cdot K_{r e} \cdot\left(\cos \phi_{2}-\cos \phi_{1}\right)+ \\
{\left[\begin{array}{l}
2 \cdot K_{t c} \cdot f_{z}-4 \cdot K_{r c} \cdot \rho \cdot \sin (\alpha-\lambda)- \\
4 \cdot K_{t c} \cdot \rho \cdot \cos (\alpha-\lambda)
\end{array}\right] \cdot\left(\phi_{2}-\phi_{1}\right)+} \\
{\left[\begin{array}{l}
K_{r c} \cdot f_{z}-2 \cdot K_{t c} \cdot \rho \cdot \sin (\alpha-\lambda)- \\
2 \cdot K_{r c} \cdot \rho \cdot \cos (\alpha-\lambda)
\end{array}\right] \cdot\left(\cos 2 \phi_{1}-\cos 2 \phi_{2}\right)+} \\
{\left[\begin{array}{l}
K_{t c} \cdot f_{z}+2 \cdot K_{r c} \cdot \rho \cdot \cos (\alpha-\lambda)- \\
2 \cdot K_{t c} \cdot \rho \cdot \sin (\alpha-\lambda)
\end{array}\right] \cdot\left(\sin 2 \phi_{2}-\cos 2 \phi_{1}\right)}
\end{array}\right]
$$

\subsubsection{Efecto tamaño}

El efecto tamaño provocará que el diente 2 no empiece a cortar hasta que el espesor de viruta supere el valor crítico. Debido al gran valor de la desviación excéntrica en algunos casos puede ocurrir que este al inicio del corte el espesor de viruta no supere este valor crítico, por lo que será necesario calcular un ángulo de entrada para el diente 2. De la misma manera que se hizo para el fresado en oposición será necesario calcular los dos ángulos críticos: el de comienzo de contacto con la pieza $\alpha_{e n 0}$ y el de comienzo del corte $\alpha_{e n}$. Para ello se deberá analizar el esquema de la figura 4.9.

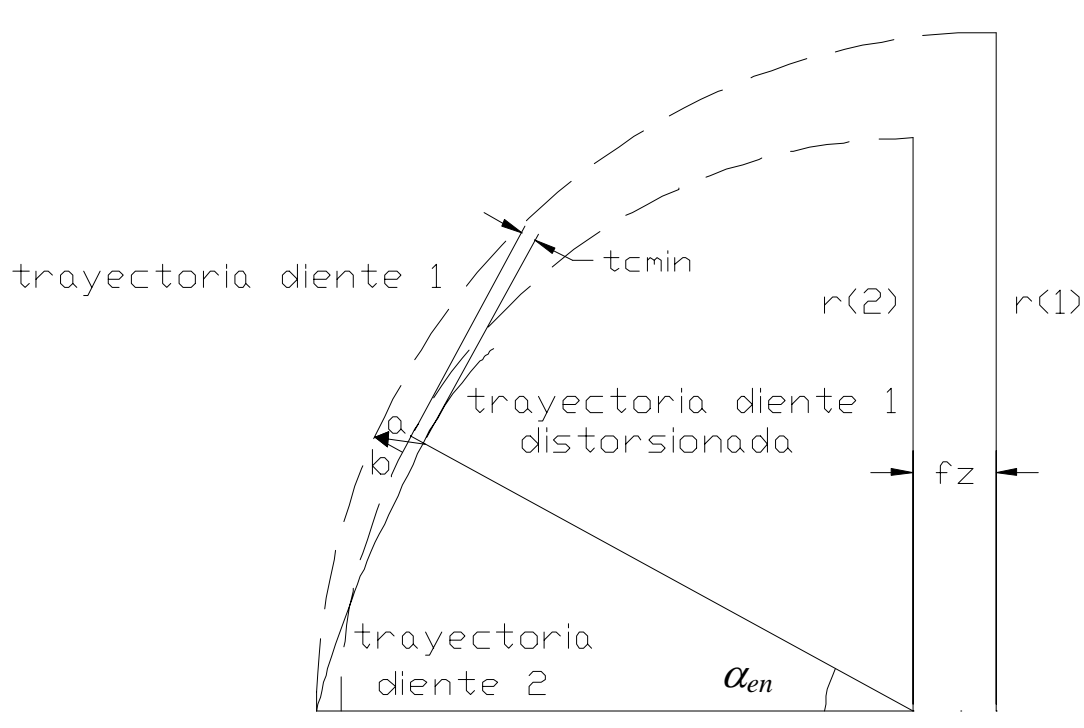

Figura 4.9. Ángulo de entrada del diente 2 en la pieza

En la figura 4.10 se muestra en detalle el esquema del espesor de viruta: 


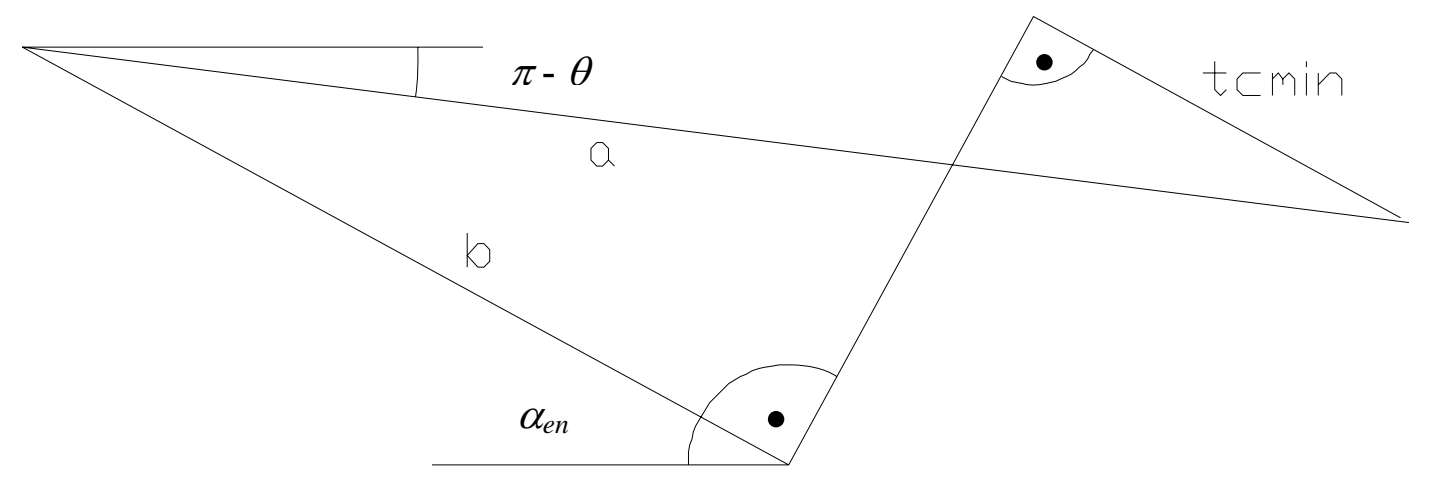

Figura 4.10. Detalle del espesor de viruta en el ángulo de entrada

Del esquema de la Figura se puede deducir la siguiente relación:

$$
-a \cdot \cos \left(\alpha_{e n}+\theta\right)=b+t_{c \min }
$$

El segmento $b$ vendrá dado por la diferencia de radios menos el espesor teórico de viruta:

$$
b=r(1)-r(2)-f_{z} \cdot \cos \alpha_{e n}
$$

El segmento $a$ se obtiene mediante la expresión (4.1):

$$
a=\left[1-\frac{K_{2}}{K_{1}} \cdot a_{p} \cdot\left(\frac{1}{2}-\frac{\alpha-\phi}{\delta}\right)\right] \cdot \frac{F_{T}(1)}{M}
$$

Si el esquema se considera aplicado al extremo inferior del diente, se deduce que:

$$
-\frac{F_{x}(1)}{M} \cdot \cos \alpha_{e n}+\frac{F_{y}(1)}{M} \cdot \sin \alpha_{e n}=2 \rho \cdot \cos \lambda-f_{z} \cdot \cos \alpha_{e n}+t_{c \min }
$$

Las fuerzas corresponderán al corte asimétrico del diente 1, ya que el diente 2 todavía no ha iniciado el corte. Los valores se pueden deducir recurriendo a las expresiones (3.56) y (3.57): 


$$
\begin{aligned}
& F_{x}(1)=\frac{D}{2 \cdot \tan \beta} \cdot\left[\begin{array}{l}
-K_{t e} \cdot\left(\cos \alpha_{e n}-1\right)-K_{r e} \cdot \sin \alpha_{e n}+ \\
\frac{f_{z}}{2} \cdot\left[-K_{t c} \cdot\left(\cos 2 \alpha_{e n}-1\right)-2 K_{r c} \cdot \alpha_{e n}-K_{r c} \cdot \sin 2 \alpha_{e n}\right]
\end{array}\right] \\
& F_{y}(1)=\frac{D}{2 \cdot \tan \beta} \cdot\left[\begin{array}{l}
K_{t e} \cdot \sin \alpha_{e n}-K_{r e} \cdot\left(\cos \alpha_{e n}-1\right)+ \\
\frac{f_{z}}{2} \cdot\left[2 K_{t c} \cdot \alpha_{e n}+K_{t c} \cdot \sin 2 \alpha_{e n}-K_{r c} \cdot\left(\cos 2 \alpha_{e n}-1\right)\right]
\end{array}\right]
\end{aligned}
$$

Sustituyendo estos valores en la expresión (4.97) se obtiene:

$$
\begin{aligned}
& \frac{D}{2 \cdot M \cdot \tan \beta} \cdot\left[\begin{array}{l}
K_{t e}-K_{t e} \cdot \cos \alpha_{e n}+K_{r e} \cdot \sin \alpha_{e n}+ \\
f_{z} \cdot\left[\begin{array}{l}
K_{r c} \cdot \alpha_{e n} \cdot \cos \alpha_{e n}+ \\
K_{r c} \cdot \sin \alpha_{e n}+K_{t c} \cdot \alpha_{e n} \cdot \sin \alpha_{e n}
\end{array}\right]
\end{array}\right]= \\
& 2 \rho \cdot \cos \lambda-f_{z} \cdot \cos \alpha_{e n}+t_{c \min }
\end{aligned}
$$

Esta ecuación deberá resolverse por métodos numéricos y proporcionará un valor para el ángulo de entrada del diente 2 en el corte.

De la misma manera se puede determinar el ángulo en el que el diente 2 empieza a tener contacto con la pieza, que constituirá el comienzo de las fuerzas de recalcado en el diente 2. Para ello se suprime el término $t_{c \min }$ en la ecuación (4.100) y se resuelve la ecuación resultante.

El efecto tamaño también provocará que el diente 2 alcance el valor crítico antes de llegar al ángulo máximo de corte, que según se ha representado en la figura 4.10, es de $90^{\circ}$, y por tanto, el filo dejará de cortar. Esto provocará que exista, igual que en el fresado en oposición, un periodo de corte asimétrico en el diente 1 y de recalcado en el diente 2. Será necesario, por tanto, calcular los ángulos de salida para cada diente y con ellos las fuerzas en cada zona. Sin embargo, el proceso de cálculo del ángulo de final del corte del diente 2 ha de ser iterativo, ya que dependerá de la posición de la herramienta y ésta de las fuerzas, que a su vez dependen del ángulo de salida. Por tanto, se programará un algoritmo iterativo que determine el ángulo partiendo de las fuerzas de corte para cada ángulo. 
Se deberá determinar también el ángulo en el que el diente 2 abandona la pieza cuando ha dejado de cortar totalmente, que constituirá, por tanto, el fin de su zona de recalcado. Se ha de suponer para ello que el ángulo de posición del extremo superior del diente es mayor que el ángulo en el que el diente 2 deja de cortar, $\alpha_{e x}$. En estas condiciones la trayectoria del diente 2 no se verá distorsionada por las fuerzas de corte, mientras que sobre la trayectoria del diente 1 actuará la distorsión procedente de las fuerzas de corte asimétrico y de recalcado. Esta situación se representa en la figura 4.11.

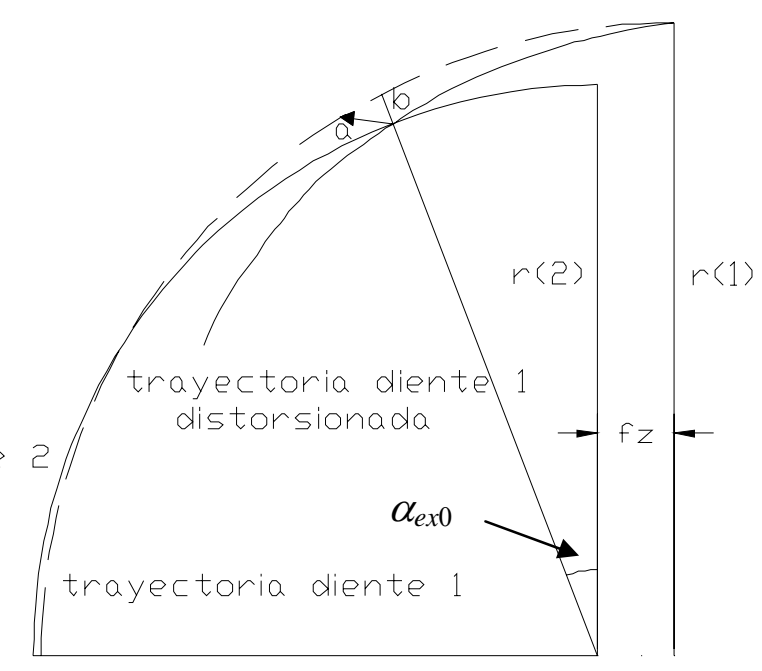

Figura 4.11. Esquema del ángulo de salida de la pieza del diente 2

En la figura 4.12 se detalla la geometría del espesor de viruta:

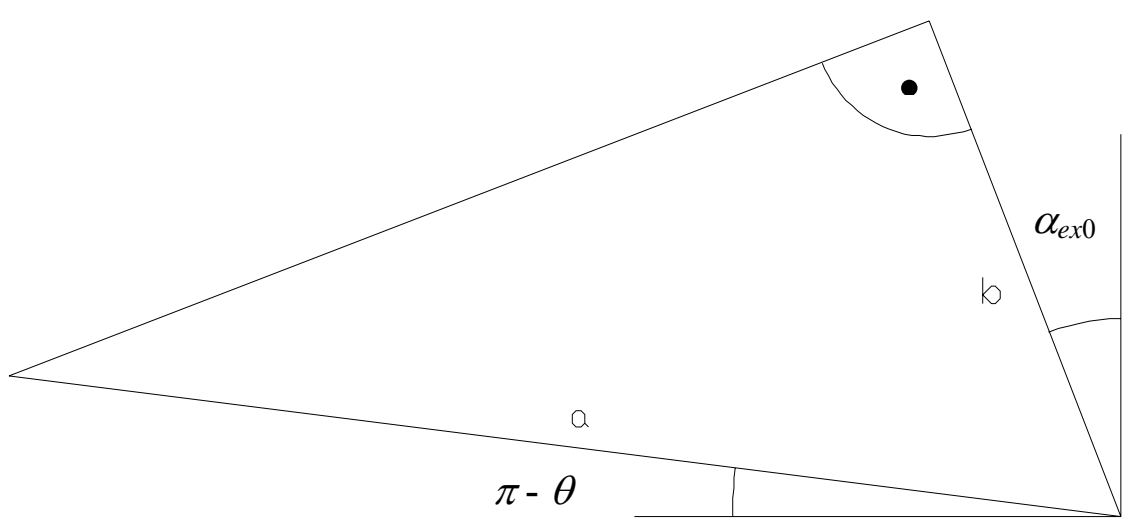

Figura 4.12. Esquema detallado del ángulo de salida 
Partiendo del esquema de la figura 5.5 se puede establecer la siguiente relación:

$$
a \cdot \cos \left(\theta_{1}-\alpha_{e x 0}-\frac{\pi}{2}\right)=b
$$

Donde $a$ viene dado por la expresión (4.1):

$$
a=\left[1-\frac{K_{2}}{K_{1}} \cdot a_{p} \cdot\left(\frac{1}{2}-\frac{\alpha-\phi}{\delta}\right)\right] \cdot \frac{F_{T}(1)}{M}
$$

La distancia $b$ se expresará de la siguiente manera:

$$
b=r(1)-r(2)-f_{z} \cdot \cos \phi
$$

De acuerdo a la expresión (3.39) se obtiene el valor de $b$ :

$$
b=2 \rho \cdot \cos (\alpha-\phi-\lambda)-f_{z} \cdot \cos \phi
$$

Sustituyendo estos valores en la expresión (5.39) y desarrollándola se obtiene:

$$
\begin{aligned}
& \frac{1}{M} \cdot\left[1-\frac{K_{2}}{K_{1}} \cdot a_{p} \cdot\left(\frac{1}{2}-\frac{\alpha-\alpha_{e x 0}}{\delta}\right)\right] \cdot\left[-F_{x}(1) \cdot \sin \alpha_{e x 0}+F_{y}(1) \cdot \cos \alpha_{e x 0}\right]= \\
& 2 \cdot \rho \cdot \cos \left(\alpha-\alpha_{e x 0}-\lambda\right)-f_{z} \cdot \cos \alpha_{e x 0}
\end{aligned}
$$

Las fuerzas sobre el diente 1 se pueden obtener teniendo en cuenta que corresponden a la suma de las fuerzas de recalcado y las de corte asimétrico. Estas fuerzas se pueden obtener a partir de las expresiones (3.56) y (3.57) estableciendo los límites de cada zona de corte:

Corte asimétrico: $\left[\alpha-\delta, \alpha_{a c}\right]$

Recalcado: $\left[\alpha_{a c}, \pi / 2\right]$ 


$$
\begin{aligned}
& F_{a c x}(1)=\frac{D}{2 \cdot \tan \beta} \cdot\left[\begin{array}{l}
-K_{t e} \cdot\left(\cos \alpha_{a c}-\cos (\alpha-\delta)\right)-K_{r e} \cdot\left(\sin \alpha_{a c}-\sin (\alpha-\delta)\right)+ \\
\frac{f_{z}}{2} \cdot\left[\begin{array}{l}
-K_{t c} \cdot\left(\cos 2 \alpha_{a c}-\cos 2(\alpha-\delta)\right)- \\
2 K_{r c} \cdot\left(\alpha_{a c}-(\alpha-\delta)\right)- \\
K_{r c} \cdot\left(\sin 2 \alpha_{a c}-\sin 2(\alpha-\delta)\right)
\end{array}\right]
\end{array}\right] \\
& F_{a c y}(1)=\frac{D}{2 \cdot \tan \beta} \cdot\left[\begin{array}{l}
K_{t e} \cdot\left(\sin \alpha_{a c}-\sin (\alpha-\delta)\right)-K_{r e} \cdot\left(\cos \alpha_{a c}-\cos (\alpha-\delta)\right)+ \\
\frac{f_{z}}{2} \cdot\left[\begin{array}{l}
2 K_{t c} \cdot\left(\alpha_{a c}-(\alpha-\delta)\right)+ \\
K_{t c} \cdot\left(\sin 2 \alpha_{a c}-\sin 2(\alpha-\delta)\right)- \\
K_{r c} \cdot\left(\cos 2 \alpha_{a c}-\cos 2(\alpha-\delta)\right)
\end{array}\right]
\end{array}\right] \\
& F_{r x}(1)=\frac{D}{2 \cdot \tan \beta} \cdot\left[K_{t e} \cdot \cos \alpha_{a c}-K_{r e} \cdot\left(1-\sin \alpha_{a c}\right)\right] \\
& F_{r y}(1)=\frac{D}{2 \cdot \tan \beta} \cdot\left[K_{t e} \cdot\left(1-\sin \alpha_{a c}\right)+K_{r e} \cdot \cos \alpha_{a c}\right]
\end{aligned}
$$

Con estos valores se puede resolver la ecuación (4.104) para cada valor de $\alpha$ mediante métodos numéricos.

El ángulo límite de corte asimétrico se puede determinar de igual manera que se hizo para el fresado en oposición partiendo de las expresiones (3.26) y (4.46):

$$
\alpha_{a c}=\arccos \frac{\lambda_{c} \cdot r_{e}}{2 \cdot f_{z}}
$$

\subsubsection{Resultados experimentales}

Se llevó a cabo una simulación del microfresado en concordancia con los siguientes parámetros: $D=1 \mathrm{~mm}, a_{e}=0.5 \mathrm{~mm}, a_{p}=0.25 \mathrm{~mm}, f_{z}=0.002 \mathrm{~mm}, r_{e}=0.001 \mathrm{~mm}, \beta=$ $30^{\circ}, \delta=16.54^{\circ}$. Los resultados de la predicción de fuerzas se muestran en la figura 4.13. 


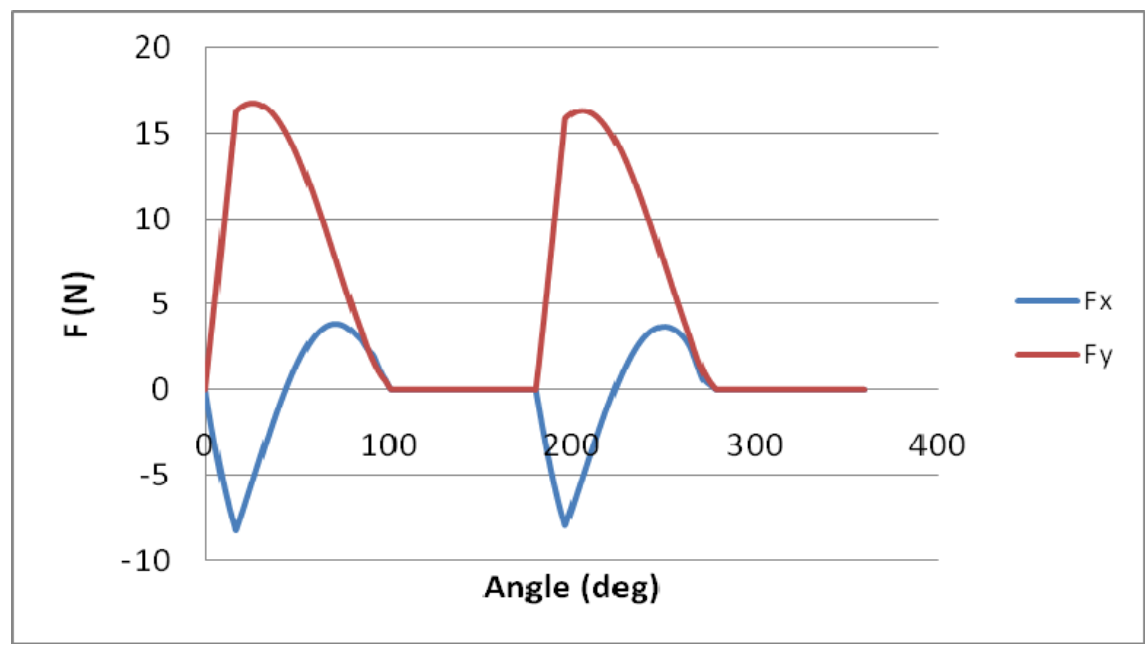

Figura 4.13. Resultados de la simulación del proceso de microfresado en concordancia

Para contrastar estos resultados del modelo se llevó a cabo un ensayo de microfresado en concordancia. Las condiciones de corte utilizadas fueron las mismas que las de la simulación mostrada en la figura 18: $D=1 \mathrm{~mm}, a_{e}=0.5 \mathrm{~mm}, a_{p}=0.25 \mathrm{~mm}, f_{z}=0.002$ $\mathrm{mm}, r_{e}=0.001 \mathrm{~mm}, \beta=30^{\circ}, \delta=16.54^{\circ}, \mathrm{n}=11141$ r.p.m. Los resultados de la medición de fuerzas se muestran en la figura 4.14 . 


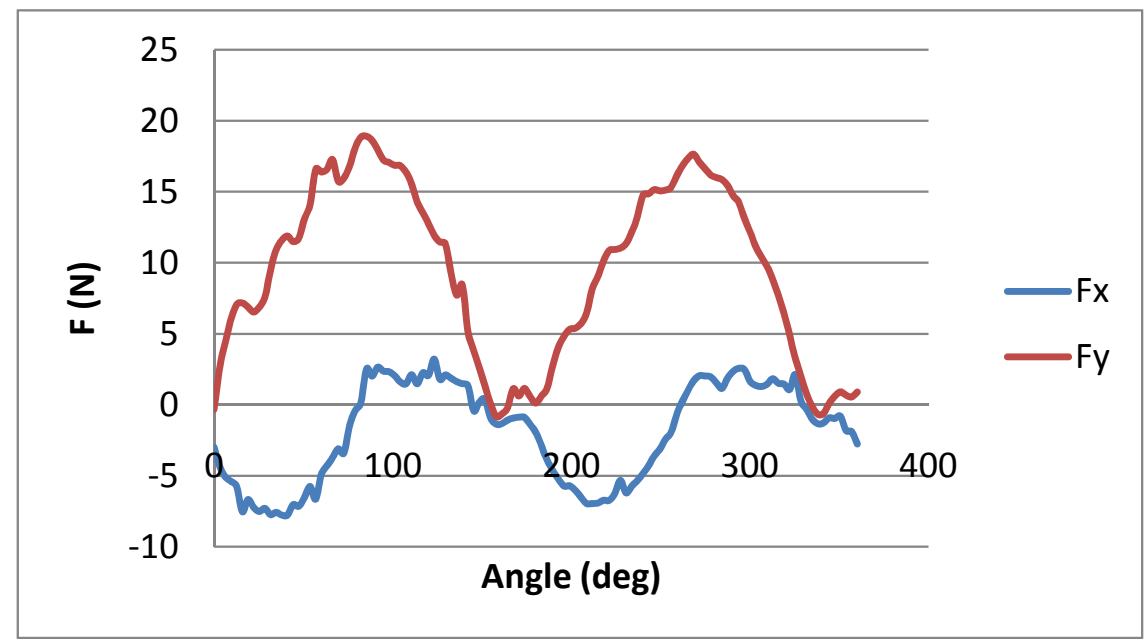

Figura 4.14. Medidas experimentales de la fuerza en microfresado en concordancia

A pesar de las vibraciones que aparecen en el proceso real, se puede observar una gran similitud cualitativa entre las dos gráficas.

Se realizó otra simulación del microfresado en concordancia con los siguientes parámetros: $M=113 \mathrm{~N} / \mathrm{mm}, D=0.4 \mathrm{~mm}, a_{e}=0.2 \mathrm{~mm}, a_{p}=0.15 \mathrm{~mm}, f_{z}=0.004 \mathrm{~mm}$, $\rho=0.003 \mathrm{~mm}, \lambda=-10^{\circ}, r_{e}=0.001 \mathrm{~mm}, \lambda_{c}=0.3, \beta=30^{\circ}, \delta=24.81^{\circ}$. Estos valores proporcionan un ángulo de salida del segundo diente de $\alpha_{e x}=91.43^{\circ}$. El ángulo de salida del primer diente es el mismo que para el segundo diente, ya que no puede haber corte asimétrico por encima de $90^{\circ}$. Los resultados de esta simulación se muestran en la figura 4.15 .

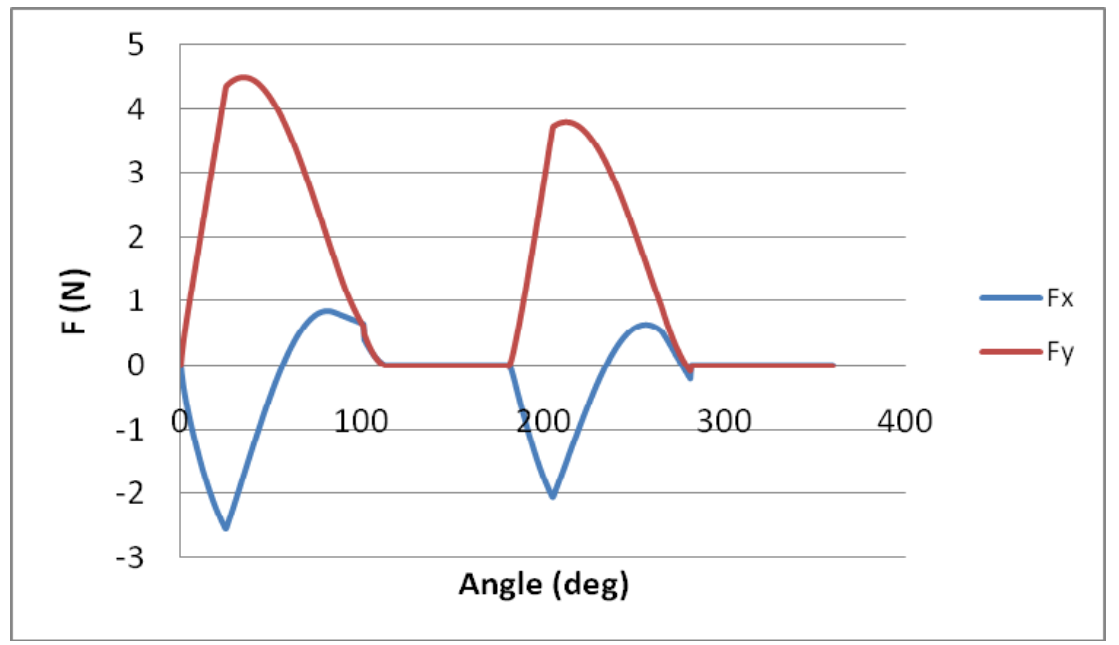


Figura 4.15. Resultados de la simulación del proceso de microfresado en concordancia

Para contrastar estas predicciones se llevó a cabo el ensayo experimental correspondiente, con los siguientes parámetros: $D=0.4 \mathrm{~mm}, a_{e}=0.2 \mathrm{~mm}, a_{p}=0.15$ $\mathrm{mm}, f_{\mathrm{z}}=0.004 \mathrm{~mm}, r_{e}=0.001 \mathrm{~mm}, \beta=30^{\circ}, \delta=24.81^{\circ}, \mathrm{n}=39789$ r.p.m. En la figura 4.16 se muestran los resultados de las mediciones de las fuerzas.

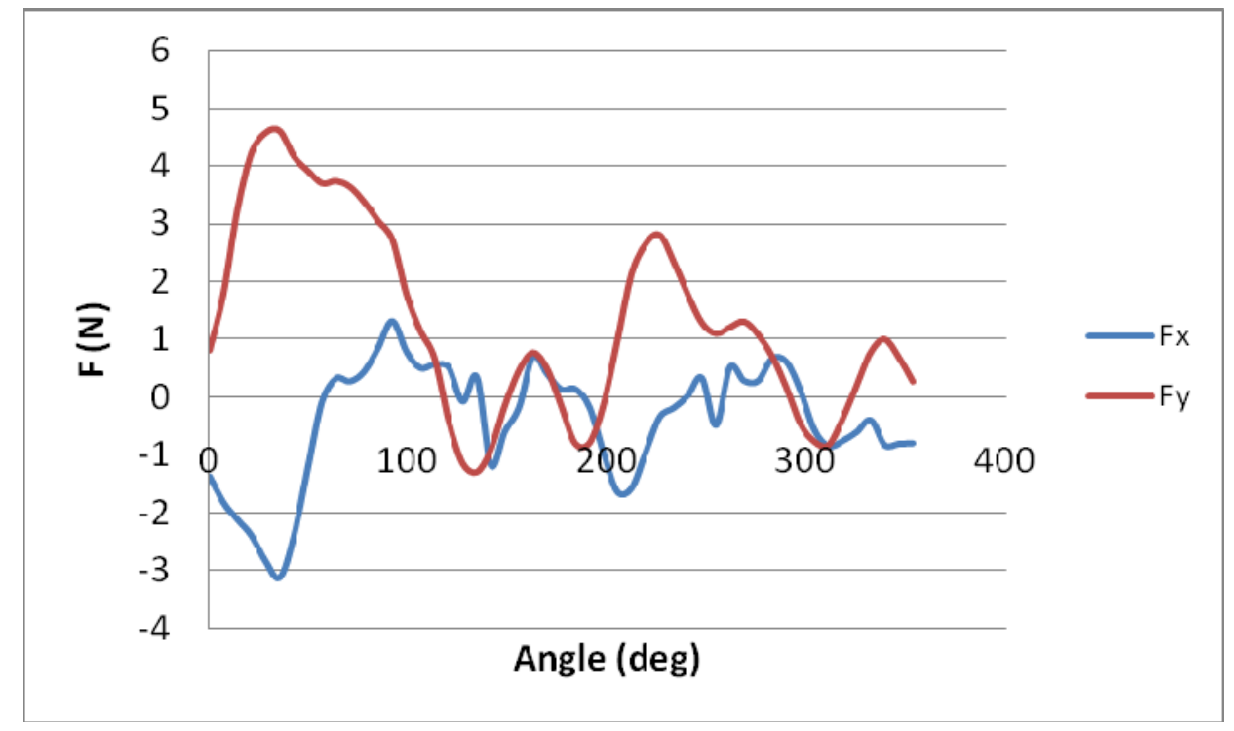

Figura 4.16. Medidas experimentales de la fuerza en microfresado en concordancia 


\section{PROGRAMACIÓN DE LA RESOLUCIÓN DEL MODELO}

\subsection{Cómputo de los diferentes tipos de fuerzas}

Dado que es necesario considerar las fuerzas de corte en los dos filos, y que la fuerza en cada filo tiene dos componentes, se considerará un vector que contenga las cuatro componentes de la fuerza:

$$
\vec{F}=\left[\begin{array}{c}
F_{x}(1) \\
F_{y}(1) \\
F_{x}(2) \\
F_{y}(2)
\end{array}\right]
$$

En los capítulos anteriores se han establecido las expresiones para el cálculo de fuerzas en corte simétrico y asimétrico, con las que se pueden determinar también las fuerzas de recalcado, es decir, las que se ejercen cuando la herramienta no está cortando, sino sólo comprimiendo el material de la pieza por no haber suficiente espesor de viruta como para que se produzca el corte.

Esta situación se dará cuando el filo esté en contacto con la pieza pero sin haberse iniciado el corte. Las zonas donde ocurra esta situación se deberán determinar geométricamente. Para ello se distinguirán los dos tipos de corte: en oposición y en concordancia.

\subsubsection{Fresado en oposición}

En la figura 6.1 se observan los ángulos que delimitan las distintas zonas de corte de la herramienta en fresado en oposición. A medida que los dientes vayan pasando por esas zonas comenzarán a darse los distintos tipos de fuerzas de corte o recalcado en uno de los dientes o en los dos. Habrá que tener en cuenta, por tanto, el paso de la punta del 
diente por cada uno de esos ángulos, pero también el del extremo superior del diente, ya que determinará la salida del diente de las distintas zonas.

En el origen de ángulos el diente 1 entra en contacto con la pieza, aunque no empiece el corte debido al efecto tamaño. En este punto comenzará el recalcado del diente 1. Cuando el diente alcanza el ángulo $\alpha_{a c}$ dará comienzo el corte asimétrico en el diente 1, que continuará hasta que el diente 2 haya comenzado a cortar en el ángulo $\alpha_{e n}$. El ángulo $\alpha_{e n 0}$ representa el inicio del contacto del diente 2 con la pieza, por tanto, darán comienzo las fuerzas de recalcado en ese diente. A partir del ángulo $\alpha_{e n}$ el corte será de tipo simétrico, que coexistirá con los otros tipos de fuerzas descritos hasta que el extremo superior del diente haya rebasado el ángulo $\alpha_{e n}$. El diente 1 abandona la pieza en el ángulo $\Phi_{2}$, como se indicó en el capítulo 3 .

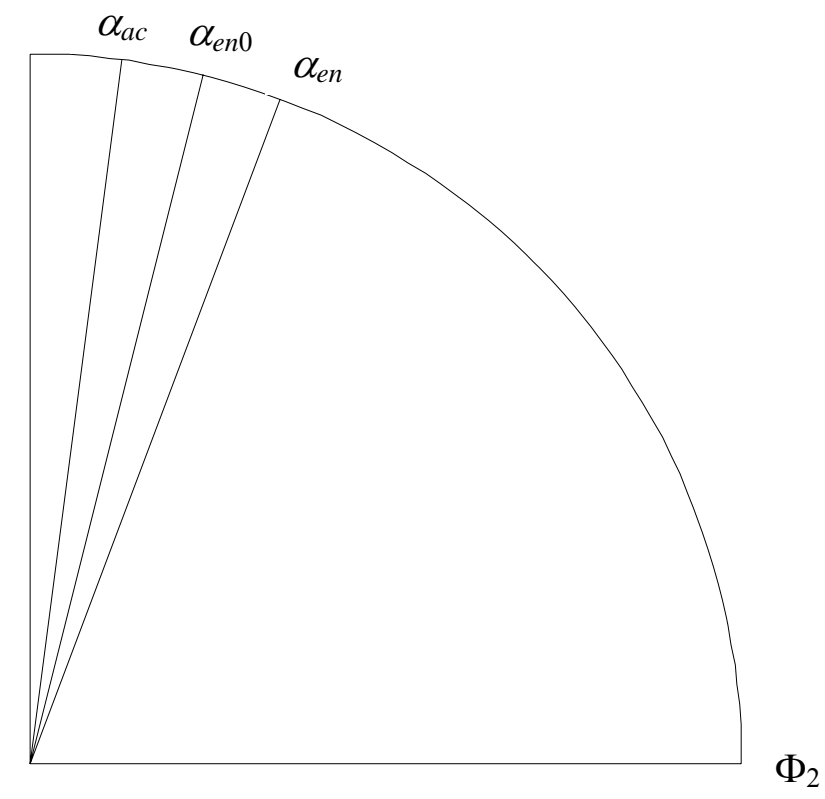

Figura 5.1. Ángulos de las distintas zonas de corte en el fresado en oposición

Para determinar las fuerzas en cada zona se deberán computar los distintos tipos de fuerzas en cada diente en la zona del filo que caiga dentro de ella. En la tabla 5.1 se indican los tipos de fuerzas que actúan en cada zona y los límites de la porción de filo implicada en cada tipo de corte. 


\begin{tabular}{|c|c|c|c|c|c|c|c|c|c|c|}
\cline { 2 - 12 } \multicolumn{1}{c|}{} & \multicolumn{5}{c|}{ DIENTE 1 } & \multicolumn{4}{c|}{ DIENTE 2 } \\
\hline \multirow{2}{*}{ Intervalo } & \multicolumn{2}{|c|}{$F_{r}$} & \multicolumn{2}{|c|}{$F_{a c}$} & \multicolumn{2}{c|}{$F_{s c}$} & \multicolumn{2}{c|}{$F_{r}$} & \multicolumn{2}{c|}{$F_{s c}$} \\
\cline { 2 - 13 } & Linf & Lsup & Linf & Lsup & Linf & Lsup & Linf & Lsup & Linf & Lsup \\
\hline$\left[0, \alpha_{a c}\right]$ & 0 & $\alpha$ & - & - & - & - & - & - & - & - \\
\hline$\left[\alpha_{a c}, \alpha_{e n 0}\right]$ & 0 & $\alpha_{a c}$ & $\alpha_{a c}$ & $\alpha$ & - & - & - & - & - & - \\
\hline$\left[\alpha_{e n 0}, \alpha_{e n}\right]$ & 0 & $\alpha_{a c}$ & $\alpha_{a c}$ & $\alpha$ & - & - & $\alpha_{e n 0}$ & $\alpha$ & - & - \\
\hline$\left[\alpha_{e n}, \delta\right]$ & 0 & $\alpha_{a c}$ & $\alpha_{a c}$ & $\alpha_{e n}$ & $\alpha_{e n}$ & $\alpha$ & $\alpha_{e n 0}$ & $\alpha_{e n}$ & $\alpha_{e n}$ & $\alpha$ \\
\hline$\left[\delta, \delta+\alpha_{a c}\right]$ & $\alpha-\delta$ & $\alpha_{a c}$ & $\alpha_{a c}$ & $\alpha_{e n}$ & $\alpha_{e n}$ & $\alpha$ & $\alpha_{e n 0}$ & $\alpha_{e n}$ & $\alpha_{e n}$ & $\alpha$ \\
\hline$\left[\delta+\alpha_{a c}, \delta+\alpha_{e n 0}\right]$ & - & - & $\alpha-\delta$ & $\alpha_{e n}$ & $\alpha_{e n}$ & $\alpha$ & $\alpha_{e n 0}$ & $\alpha_{e n}$ & $\alpha_{e n}$ & $\alpha$ \\
\hline$\left[\delta+\alpha_{e n 0}, \delta+\alpha_{e n}\right]$ & - & - & $\alpha-\delta$ & $\alpha_{e n}$ & $\alpha_{e n}$ & $\alpha$ & $\alpha-\delta$ & $\alpha_{e n}$ & $\alpha_{e n}$ & $\alpha$ \\
\hline$\left[\delta+\alpha_{e n}, \Phi_{2}\right]$ & - & - & - & - & $\alpha-\delta$ & $\alpha$ & - & - & $\alpha-\delta$ & $\alpha$ \\
\hline$\left[\Phi_{2}, \delta+\Phi_{2}\right]$ & - & - & - & - & $\alpha-\delta$ & $\Phi_{2}$ & - & - & $\alpha-\delta$ & $\Phi_{2}$ \\
\hline
\end{tabular}

Tabla 5.1. Límites de las distintas zonas de corte del fresado en oposición

En general la fuerza en el proceso se puede expresar como suma de todos los tipos de fuerzas en los dos dientes:

$$
\vec{F}(\alpha)=\overrightarrow{F_{r}}+\overrightarrow{F_{a c}}+\overrightarrow{F_{s c}}
$$

De esta expresión habrá que eliminar los términos que no aparezcan según la zona en la que se encuentre la herramienta.

La expresión general de las fuerzas de recalcado es la correspondiente al corte en condiciones ideales suprimiendo los términos que multiplican al espesor de viruta. Partiendo de las expresiones (3.12) y (3.13) se obtiene:

$$
F_{x}=\frac{D}{2 \cdot \tan \beta} \cdot\left[K_{t e} \cdot\left(\sin \phi_{2}-\sin \phi_{1}\right)-K_{r e} \cdot\left(\cos \phi_{2}-\cos \phi_{1}\right)\right]
$$




$$
F_{y}=\frac{D}{2 \cdot \tan \beta} \cdot\left[K_{t e} \cdot\left(\cos \phi_{2}-\cos \phi_{1}\right)+K_{r e} \cdot\left(\sin \phi_{2}-\sin \phi_{1}\right)\right]
$$

Donde $\phi_{1}$ y $\phi_{2}$ son los límites de integración. Esta expresión es válida para ambos dientes.

En cuanto a las fuerzas de corte asimétrico en el diente 1 vienen dadas directamente por las expresiones (3.49) y (3.50):

$$
\begin{aligned}
& F_{x}(1)=\frac{D}{2 \cdot \tan \beta} \cdot\left[\begin{array}{l}
K_{t e} \cdot\left(\sin \phi_{2}-\sin \phi_{1}\right)-K_{r e} \cdot\left(\cos \phi_{2}-\cos \phi_{1}\right)+ \\
\frac{f_{z}}{2} \cdot\left[\begin{array}{l}
-K_{t c} \cdot\left(\cos 2 \phi_{2}-\cos 2 \phi_{1}\right)+2 K_{r c} \cdot\left(\phi_{2}-\phi_{1}\right)- \\
K_{r c} \cdot\left(\sin 2 \phi_{2}-\sin 2 \phi_{1}\right)
\end{array}\right]
\end{array}\right] \\
& F_{y}(1)=\frac{D}{2 \cdot \tan \beta} \cdot\left[\begin{array}{l}
K_{t e} \cdot\left(\cos \phi_{2}-\cos \phi_{1}\right)+K_{r e} \cdot\left(\sin \phi_{2}-\sin \phi_{1}\right)+ \\
\frac{f_{z}}{2} \cdot\left[\begin{array}{l}
-2 K_{t c} \cdot\left(\phi_{2}-\phi_{1}\right)+K_{t c} \cdot\left(\sin 2 \phi_{2}-\sin 2 \phi_{1}\right)- \\
K_{r c} \cdot\left(\cos 2 \phi_{2}-\cos 2 \phi_{1}\right)
\end{array}\right]
\end{array}\right]
\end{aligned}
$$

Las fuerzas de corte simétrico se calcularán mediante el método iterativo que se explica más adelante.

El procedimiento para la simulación de fuerzas consiste en un cálculo punto a punto del sistema de fuerzas mediante un proceso iterativo en el que dependiendo de la posición de los dientes se computa un tipo de fuerzas u otro.

En una vuelta completa de la herramienta se pueden distinguir las siguientes zonas, según se deduce de la tabla 5.1:

1. $\left[0, \alpha_{a c}\right]$ : Recalcado del diente 1

Cálculo de las fuerzas de recalcado mediante las expresiones arriba vistas

2. $\left[\alpha_{a c}, \alpha_{e n 0}\right]$ : Corte asimétrico

Suma de las fuerzas de recalcado más las fuerzas de corte asimétrico, proporcionadas por las fórmulas (3.49) y (3.50) 
3. $\left[\alpha_{e n 0}, \alpha_{e n}\right]:$ Recalcado del diente 2

Suma de las fuerzas anteriores más la fuerza de recalcado en el diente 2, obtenido mediante las expresiones (5.3) y (5.4)

4. $\left[\alpha_{e n}, \alpha_{e n}+\delta\right]$ : Entrada del diente 2 en el corte

Actualización del valor $\alpha_{e n}$, actualización del valor $\alpha_{e n 0}$, cálculo de la suma de fuerzas de corte (recalcado diente 1, corte asimétrico, recalcado diente 2, corte simétrico) con los ángulos anteriormente calculados. Las fuerzas de corte simétrico se calcularán mediante los sistemas de ecuaciones (4.61) y (4.62)

5. $\left[\alpha_{e n}+\delta, \Phi_{2}\right]:$ Corte simétrico

Cálculo de las fuerzas simétricas mediante el sistema (4.21)

6. $\left[\Phi_{2}, \delta+\Phi_{2}\right]$ : Salida de dientes

Cálculo de las fuerzas simétricas considerando un ángulo máximo igual a $\Phi_{2}$

En la figura 5.2 se muestra el algoritmo de programación utilizado para calcular el ángulo de entrada $\alpha_{e n}$. Se trata de un método de cálculo iterativo, como se indicó en el capítulo 4, que requiere por tanto un valor inicial y un cómputo de los diferentes tipos de fuerzas.

Para el cálculo del ángulo de contacto $\alpha_{e n 0}$ se seguiría un procedimiento similar. 


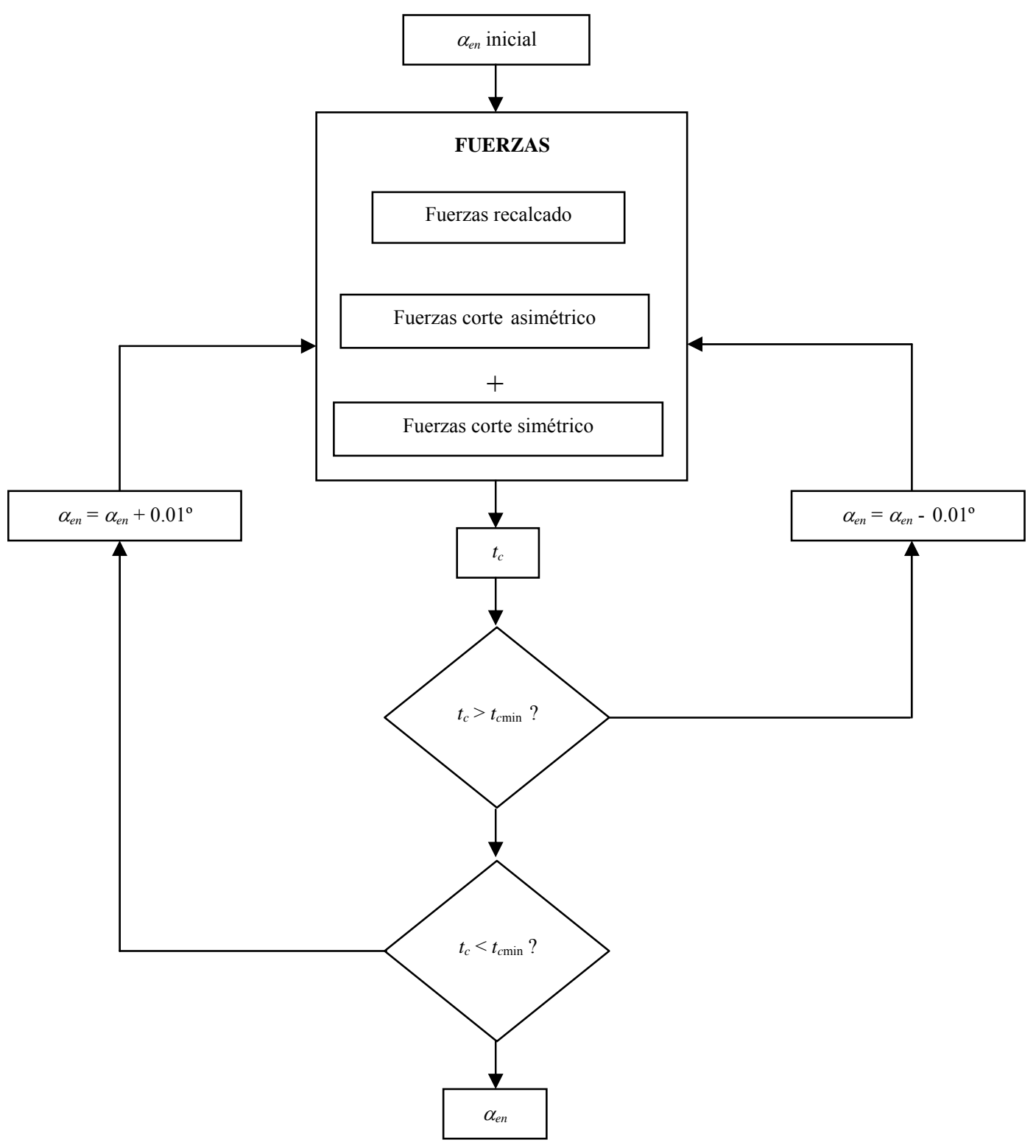

Figura 5.2. Algoritmo de cálculo del ángulo de entrada

\subsubsection{Fresado en concordancia}

En el fresado en concordancia se puede considerar que la herramienta entra en el corte en el ángulo de posición $\Phi_{1}$ determinado geométricamente mediante la expresión:

$$
\Phi_{1}=\arcsin \left(1-\frac{2 \cdot a_{e}}{D}\right)
$$




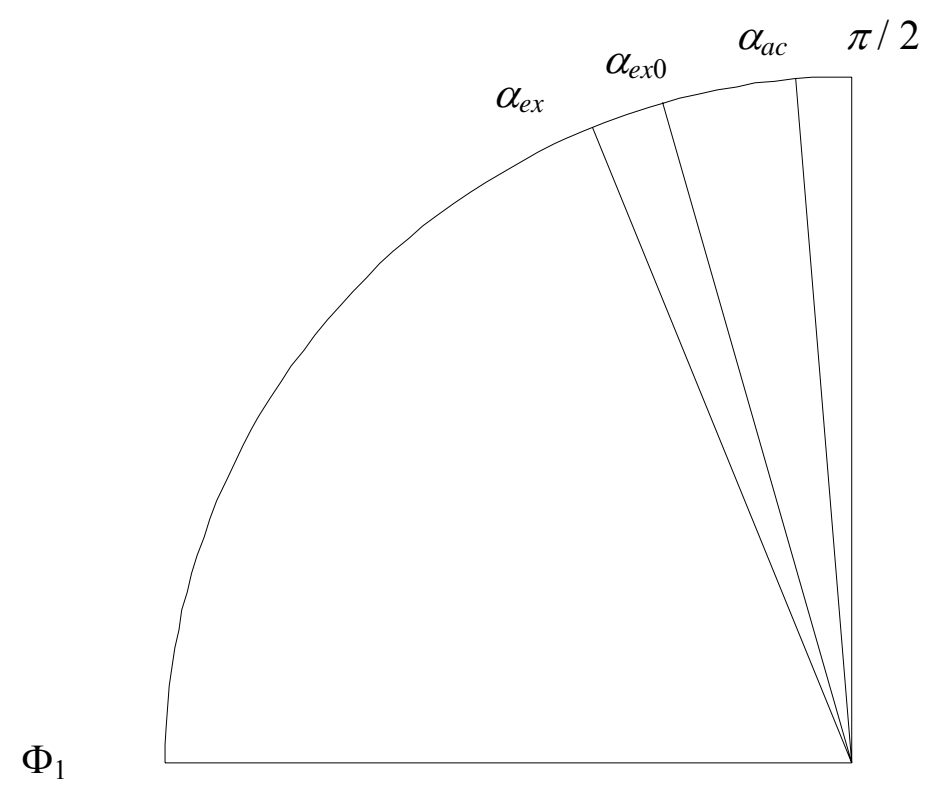

Figura 5.3. Ángulos de las distintas zonas de corte en el fresado en concordancia

Sin embargo, abandonará la pieza antes de que la posición del filo corresponda al ángulo máximo $\pi / 2$, ya que la desviación excéntrica de la herramienta distorsionará la trayectoria de los filos respecto a la teórica. La salida del filo pasará por varias fases, igual que la entrada en el fresado en oposición. Primero dejará de cortar el diente 2 debido al efecto tamaño en el ángulo $\alpha_{e x}$. A partir de este ángulo existirán fuerzas de corte asimétrico en el diente 1 y fuerzas de recalcado en el diente 2. Posteriormente el diente 2 dejará de tener contacto con la pieza en el ángulo $\alpha_{e x 0}$. En ese momento desaparecerán las fuerzas de recalcado y se mantendrán únicamente las de corte asimétrico en el diente 1. Cuando el diente alcance el ángulo $\alpha_{a c}$ desaparecerán las fuerzas de corte asimétrico y darán comienzo las fuerzas de recalcado en el diente 1, que se mantendrán hasta el ángulo de salida teórico $\pi / 2$. En la Figura 12 se indica la posición de los ángulos de salida de los dientes en el fresado en concordancia.

En la tabla 5.2 se señalan los tipos de fuerzas que actúan en cada zona y los límites de la porción de filo implicada en cada tipo de corte. 


\begin{tabular}{|c|c|c|c|c|c|c|c|c|c|c|}
\cline { 2 - 13 } \multicolumn{1}{c|}{} & \multicolumn{5}{c|}{ DIENTE 1 } & \multicolumn{3}{c|}{ DIENTE 2} \\
\hline \multirow{2}{*}{ Intervalo } & \multicolumn{2}{|c|}{$F_{r}$} & \multicolumn{2}{|c|}{$F_{a c}$} & \multicolumn{2}{|c|}{$F_{s c}$} & \multicolumn{2}{|c|}{$F_{r}$} & \multicolumn{2}{c|}{$F_{s c}$} \\
\cline { 2 - 14 } & Linf & Lsup & Linf & Lsup & Linf & Lsup & Linf & Lsup & Linf & Lsup \\
\hline$\left[\Phi_{1}, \Phi_{1}+\delta\right]$ & - & - & - & - & $\Phi_{1}$ & $\alpha$ & - & - & $\Phi_{1}$ & $\alpha$ \\
\hline$\left[\Phi_{1}+\delta, \alpha_{e x}\right]$ & - & - & - & - & $\alpha-\delta$ & $\alpha$ & - & - & $\alpha-\delta$ & $\alpha$ \\
\hline$\left[\alpha_{e x}, \alpha_{e x 0}\right]$ & - & - & $\alpha_{e x}$ & $\alpha$ & $\alpha-\delta$ & $\alpha_{e x}$ & $\alpha_{e x}$ & $\alpha$ & $\alpha-\delta$ & $\alpha_{e x}$ \\
\hline$\left[\alpha_{e x 0}, \alpha_{a c}\right]$ & - & - & $\alpha_{e x}$ & $\alpha$ & $\alpha-\delta$ & $\alpha_{e x}$ & $\alpha_{e x}$ & $\alpha_{e x 0}$ & $\alpha-\delta$ & $\alpha_{e x}$ \\
\hline$\left[\alpha_{a c}, \pi / 2\right]$ & $\alpha_{a c}$ & $\alpha$ & $\alpha_{e x}$ & $\alpha_{a c}$ & $\alpha-\delta$ & $\alpha_{e x}$ & $\alpha_{e x}$ & $\alpha_{e x 0}$ & $\alpha-\delta$ & $\alpha_{e x}$ \\
\hline$\left[\pi / 2, \alpha_{e x}+\delta\right]$ & $\alpha_{a c}$ & $\pi / 2$ & $\alpha_{e x}$ & $\alpha_{a c}$ & $\alpha-\delta$ & $\alpha_{e x}$ & $\alpha_{e x}$ & $\alpha_{e x 0}$ & $\alpha-\delta$ & $\alpha_{e x}$ \\
\hline$\left[\alpha_{e x}+\delta, \alpha_{e x 0}+\delta\right]$ & $\alpha_{a c}$ & $\pi / 2$ & $\alpha-\delta$ & $\alpha_{a c}$ & - & - & $\alpha-\delta$ & $\alpha_{e x 0}$ & - & - \\
\hline$\left[\alpha_{e x 0}+\delta, \alpha_{a c}+\delta\right]$ & $\alpha_{a c}$ & $\pi / 2$ & $\alpha-\delta$ & $\alpha_{a c}$ & - & - & - & - & - & - \\
\hline$\left[\alpha_{a c}+\delta, \pi / 2+\delta\right]$ & $\alpha-\delta$ & $\pi / 2$ & - & - & - & - & - & - & - & - \\
\hline
\end{tabular}

Tabla 5.2. Límites de las distintas zonas de corte del fresado en concordancia

En este proceso las fuerzas de recalcado se deducirán de las expresiones (3.28) y (3.29) suprimiendo los términos que multiplican al espesor de viruta:

$$
\begin{aligned}
& F_{x}=\frac{D}{2 \cdot \tan \beta} \cdot\left[-K_{t e} \cdot\left(\cos \phi_{2}-\cos \phi_{1}\right)-K_{r e} \cdot\left(\sin \phi_{2}-\sin \phi_{1}\right)\right] \\
& F_{y}=\frac{D}{2 \cdot \tan \beta} \cdot\left[K_{t e} \cdot\left(\sin \phi_{2}-\sin \phi_{1}\right)-K_{r e} \cdot\left(\cos \phi_{2}-\cos \phi_{1}\right)\right]
\end{aligned}
$$

Las fuerzas de corte asimétrico vienen dadas por las expresiones (3.56) y (3.57):

$$
F_{x}(1)=\frac{D}{2 \cdot \tan \beta} \cdot\left[\begin{array}{l}
-K_{t e} \cdot\left(\cos \phi_{2}-\cos \phi_{1}\right)-K_{r e} \cdot\left(\sin \phi_{2}-\sin \phi_{1}\right)+ \\
\frac{f_{z}}{2} \cdot\left[\begin{array}{l}
-K_{t c} \cdot\left(\cos 2 \phi_{2}-\cos 2 \phi_{1}\right)-2 K_{r c} \cdot\left(\phi_{2}-\phi_{1}\right)- \\
K_{r c} \cdot\left(\sin 2 \phi_{2}-\sin 2 \phi_{1}\right)
\end{array}\right]
\end{array}\right]
$$




$$
F_{y}(1)=\frac{D}{2 \cdot \tan \beta} \cdot\left[\begin{array}{l}
K_{t e} \cdot\left(\sin \phi_{2}-\sin \phi_{1}\right)-K_{r e} \cdot\left(\cos \phi_{2}-\cos \phi_{1}\right)+ \\
\frac{f_{z}}{2} \cdot\left[\begin{array}{l}
2 K_{t c} \cdot\left(\phi_{2}-\phi_{1}\right)+K_{t c} \cdot\left(\sin 2 \phi_{2}-\sin 2 \phi_{1}\right)- \\
K_{r c} \cdot\left(\cos 2 \phi_{2}-\cos 2 \phi_{1}\right)
\end{array}\right]
\end{array}\right]
$$

Con los valores de las fuerzas determinados mediante las expresiones anteriores y mediante los sistemas de ecuaciones (4.21) ó (4.61) y (4.62) se llevaría a cabo el algoritmo mostrado en la figura 5.2 para determinar $\alpha_{e x}$ ó $\alpha_{e x 0}$. 


\section{DETERMINACIÓN DE LOS PARÁMETROS DEL MODELO}

\subsection{Ensayo de fuerza constante}

El modelo analítico de fuerzas requiere un procedimiento para dar valor a los parámetros que se utilizan en las simulaciones. Algunos de ellos son conocidos como el diámetro de la herramienta, el avance por diente, el radio del filo, la constante de elasticidad de la herramienta, el ángulo de hélice y las profundidades radial y axial de corte. El valor del parámetro $\lambda_{c}$ se tomará de estudios experimentales realizados sobre el efecto tamaño [70]. El resto son desconocidos y deberán obtenerse de tal manera que la señal de fuerzas que proporciona la simulación sea igual que la señal de fuerzas medidas en el proceso real.

En trabajos anteriores se ha estimado la constante $K$ de las fuerzas calculando el valor medio de las fuerzas experimentales e igualándolo a la predicción del modelo [37]. En otros casos se han utilizado los datos experimentales para obtener una expresión que proporcione la constante $K$ en función del espesor medio de la viruta [72].

Existe una combinación de parámetros para la que la fuerza del proceso es constante, que corresponde al caso en el que toda la superficie de contacto entre la fresa y la pieza está siendo cortada y no hay solapes entre dientes. Con estas condiciones en un caso ideal, es decir, sin desviación excéntrica ni deflexión de herramienta, la gráfica de fuerzas sería una recta horizontal. Sin embargo, al existir desviaciones existirá una cierta ondulación en las fuerzas. Pese a esta distorsión se puede considerar que el valor medio de estas fuerzas será muy aproximado al valor teórico del proceso ideal. Dado que este valor teórico dependerá sólo de los coeficientes de las fuerzas $K_{t e}, K_{t c}, K_{r e} \mathrm{y} K_{r c}$ se puede estimar fácilmente el valor de estos parámetros identificando el valor de las fuerzas medido con la expresión que proporciona el modelo en función de estos coeficientes.

La condición para que las fuerzas sean constantes es [36]: 


$$
Z \cdot a_{p} \cdot \tan \beta=n \cdot \pi \cdot D, n=1,2,3 \ldots
$$

El caso más asequible desde el punto de vista práctico es el correspondiente a $n=1$. Las expresiones de la fuerza para este caso concreto se deducirán teniendo en cuenta que en todo momento está siendo cortada toda la superficie expuesta al corte, ya que cuando un diente abandona la pieza por su parte superior inmediatamente aparece el siguiente diente cortando por la parte inferior. En este tipo de corte sólo hay, por tanto, una fase y la fuerza se obtendrá integrando el diferencial de fuerza entre el ángulo inicial y el final de la zona de corte.

Dado que se pretenden estimar 4 coeficientes de fuerzas, se necesitan 4 ecuaciones, que se obtendrán de igualar las expresiones proporcionadas por el modelo ideal a cada una de las fuerzas presentes en el fenómeno. Las fuerzas del proceso pueden descomponerse en fuerza debida al filo y fuerza debida al corte en dependencia lineal con el avance por diente, de modo análogo a como se hizo en las expresiones (3.1) y (3.2):

$$
\begin{aligned}
& F_{x}=F_{x e}+f_{z} \cdot F_{x c} \\
& F_{y}=F_{y e}+f_{z} \cdot F_{y c}
\end{aligned}
$$

Los datos experimentales de fuerzas medias se pueden ajustar a esas relaciones mediante regresión lineal, obteniéndose así los parámetros $F_{x e}, F_{x c}, F_{y e}$ y $F_{y c}$ como se expone en [41].

A continuación se desarrollarán las expresiones de las fuerzas según el modelo de fresado en condiciones ideales expuesto en el capítulo 3, distinguiendo entre fresado en oposición y en concordancia.

\subsubsection{Fresado en oposición}


Mediante el modelo se puede obtener la expresión teórica de los parámetros $F_{x e}$ y $F_{y e}$ igualando a 0 el avance por diente en las expresiones (3.12) y (3.13). Con estas expresiones se pueden deducir los coeficientes de las fuerzas de recalcado $K_{t e}$ y $K_{r e}$ :

$$
\begin{aligned}
& d F_{x e}=\frac{D}{2 \cdot \tan \beta} \cdot\left(K_{t e} \cdot \cos \phi+K_{r e} \cdot \sin \phi\right) \cdot d \phi \\
& d F_{y e}=\frac{D}{2 \cdot \tan \beta} \cdot\left(-K_{t e} \cdot \sin \phi+K_{r e} \cdot \cos \phi\right) \cdot d \phi
\end{aligned}
$$

Integrando estas expresiones a lo largo de todo el filo se obtiene:

$$
\begin{aligned}
& F_{x e}=\frac{D}{2 \cdot \tan \beta} \cdot\left(K_{t e} \cdot \sin \Phi_{2}-K_{r e} \cdot \cos \Phi_{2}+K_{r e}\right) \\
& F_{y e}=\frac{D}{2 \cdot \tan \beta} \cdot\left(K_{t e} \cdot \cos \Phi_{2}-K_{t e}+K_{r e} \cdot \sin \Phi_{2}\right)
\end{aligned}
$$

De aquí se pueden despejar los coeficientes $K_{t e}$ y $K_{r e}$ :

$$
\begin{gathered}
K_{t e}=\frac{\tan \beta}{D} \cdot \frac{F_{x e} \cdot \sin \Phi_{2}+F_{y e} \cdot\left(\cos \Phi_{2}-1\right)}{1-\cos \Phi_{2}} \\
K_{r e}=\frac{K_{t e} \cdot \sin \Phi_{2}-F_{x e} \cdot \frac{2 \cdot \tan \beta}{D}}{\cos \Phi_{2}-1}
\end{gathered}
$$

Las expresiones de las fuerzas en condiciones de fuerza constante coincidirán con las que se obtuvieron para la fase B del fresado tipo II: (3.20) y (3.21), ya que en ese caso los límites de integración son el ángulo de entrada y salida de la fresa en la pieza. Comparando esas expresiones con las (6.6) y (6.7) se identifican las ecuaciones que proporcionan las fuerzas $F_{x c}$ y $F_{y c}$. De ellas se pueden deducir las expresiones de los coeficientes $K_{t c}$ y $K_{r c}$ : 


$$
\begin{aligned}
& F_{x c}=\frac{D}{8 \cdot \tan \beta} \cdot\left(-K_{t c} \cdot \cos 2 \Phi_{2}+K_{t c}+2 K_{r c} \cdot \Phi_{2}-K_{r c} \cdot \sin 2 \Phi_{2}\right)(6.10) \\
& F_{y c}=\frac{D}{8 \cdot \tan \beta} \cdot\left(-2 K_{t c} \cdot \Phi_{2}+K_{t c} \cdot \sin 2 \Phi_{2}-K_{r c} \cdot \cos 2 \Phi_{2}+K_{r c}\right)(6.11)
\end{aligned}
$$

De aquí se despejan los coeficientes de fuerzas:

$$
\begin{gathered}
K_{r c}=\frac{8 \cdot \tan \beta}{D} \cdot \frac{F_{x c} \cdot\left(2 \Phi_{2}-\sin 2 \Phi_{2}\right)+F_{y c} \cdot\left(\cos 2 \Phi_{2}-1\right)}{2+4 \Phi_{2}{ }^{2}-4 \Phi_{2} \cdot \sin 2 \Phi_{2}-2 \cos 2 \Phi_{2}} \\
K_{t c}=\frac{K_{r c} \cdot\left(2 \Phi_{2}-\sin 2 \Phi_{2}\right)+F_{x c} \cdot \frac{8 \cdot \tan \beta}{D}}{1-\cos 2 \Phi_{2}}(6.13)
\end{gathered}
$$

\subsubsection{Fresado en concordancia}

De igual manera que para el fresado en oposición se pueden obtener las fuerzas debidas al filo igualando a 0 el avance por diente en las expresiones (3.28) y (3.29)

$$
\begin{aligned}
& d F_{x e}=\frac{D}{2 \cdot \tan \beta} \cdot\left(K_{t e} \cdot \sin \phi-K_{r e} \cdot \cos \phi\right) \cdot d \phi \\
& d F_{y e}=\frac{D}{2 \cdot \tan \beta} \cdot\left(K_{t e} \cdot \cos \phi+K_{r e} \cdot \sin \phi\right) \cdot d \phi
\end{aligned}
$$

Integrando estas expresiones a lo largo de todo el filo se obtiene:

$$
\begin{aligned}
& F_{x e}=\frac{D}{2 \cdot \tan \beta} \cdot\left(-K_{t e} \cdot \cos \Phi_{2}+K_{t e}-K_{r e} \cdot \sin \Phi_{2}\right) \\
& F_{y e}=\frac{D}{2 \cdot \tan \beta} \cdot\left(K_{t e} \cdot \sin \Phi_{2}-K_{r e} \cdot \cos \Phi_{2}+K_{r e}\right)
\end{aligned}
$$


Despejando los coeficientes:

$$
\begin{gathered}
K_{r e}=-\frac{\tan \beta}{D} \cdot \frac{F_{x e} \cdot \sin \Phi_{2}+F_{y e} \cdot\left(\cos \Phi_{2}-1\right)}{1-\cos \Phi_{2}} \\
K_{t e}=-\frac{F_{x e} \cdot \frac{2 \cdot \tan \beta}{D}+K_{r e} \cdot \sin \Phi_{2}}{\cos \Phi_{2}-1}
\end{gathered}
$$

Comparando las expresiones (3.32) y (3.33) con las (6.2) y (6.3) se identifican las ecuaciones que proporcionan las fuerzas de corte:

$$
\begin{aligned}
& F_{x c}=\frac{D}{8 \cdot \tan \beta} \cdot\left(-K_{t c} \cdot \cos 2 \Phi_{2}+K_{t c}-2 K_{r c} \cdot \Phi_{2}-K_{r c} \cdot \sin 2 \Phi_{2}\right)(6.20) \\
& F_{y c}=\frac{D}{8 \cdot \tan \beta} \cdot\left(2 K_{t c} \cdot \Phi_{2}+K_{t c} \cdot \sin 2 \Phi_{2}-K_{r c} \cdot \cos 2 \Phi_{2}+K_{r c}\right)
\end{aligned}
$$

Despejando los coeficientes de fuerza de estas expresiones:

$$
\begin{gathered}
K_{r c}=-\frac{4 \cdot \tan \beta}{D} \cdot \frac{F_{x c} \cdot\left(2 \Phi_{2}+\sin 2 \Phi_{2}\right)+F_{y c} \cdot\left(\cos 2 \Phi_{2}-1\right)}{1-\cos 2 \Phi_{2}+2 \Phi_{2}{ }^{2}+2 \Phi_{2} \cdot \sin 2 \Phi_{2}} \\
K_{t c}=-\frac{F_{x c} \cdot \frac{8 \cdot \tan \beta}{D}+K_{r c} \cdot\left(2 \Phi_{2}+\sin 2 \Phi_{2}\right)}{\cos 2 \Phi_{2}-1}
\end{gathered}
$$

\subsection{Ensayo de fuerza variable}

Cuando no se dispone de suficiente longitud de filo como para que la profundidad axial de corte sea la requerida en la expresión (6.1), se recurre a un corte con profundidad menor para obtener una señal que aunque no es constante sí tiene un alto componente de señal continua, lo cual permite igualar este valor con el valor medio que se tendría en condiciones ideales, es decir, si no hubiese desviación excéntrica. 
Las fuerzas correspondientes al fresado en condiciones ideales se pueden obtener a partir de las expresiones (3.18)-(3.25) y (3.30)-(3.37). En este caso la profundidad axial será un valor genérico $a_{p}$. Dado que en este caso la fuerza del proceso no es constante habrá que obtener el valor medio de la fuerza en las diferentes fases del corte. Por tanto:

$$
\bar{F}=\frac{1}{\pi} \cdot \sum_{i=1}^{N} \int_{F_{i}} F \cdot d \alpha
$$

Donde $F_{i}$ es cada una de las fases en que se divide el periodo de corte de un diente. En la figura 6.1 se representa la superficie de corte desarrollada y las líneas correspondientes a los dientes.

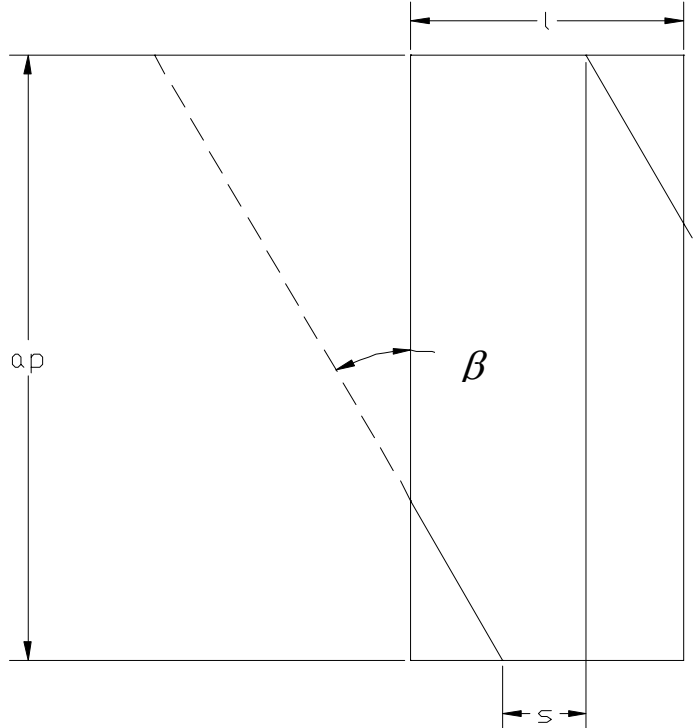

Figura 6.1. Esquema de la zona de corte

Según ese esquema habrá 4 fases en el corte:

i) Desde la entrada del diente en la zona de corte hasta la completa salida del siguiente

ii) Desde el final de la etapa anterior hasta que la punta del diente sale de la zona del corte

iii) Desde el final de la etapa anterior hasta que el extremo superior del diente entra en la zona de corte 
iv) Desde el final de la etapa anterior hasta que entra el siguiente diente en la zona de corte

Para caracterizar estas zonas se deberán localizar los puntos críticos del recorrido del diente. Es importante conocer la distancia s entre el punto superior implicado en el corte de un diente y el punto inferior del siguiente, que se expresará:

$$
s=\pi \cdot \frac{D}{2}-a_{p} \cdot \tan \beta(6.25)
$$

El ángulo $\sigma$ correspondiente a esta distancia será:

$$
\sigma=\pi-\frac{2 a_{p} \cdot \tan \beta}{D}
$$

Por otro lado, el ángulo $\delta$ que ocupa cada diente es:

$$
\delta=\frac{2 \cdot a_{p} \cdot \tan \beta}{D}
$$

Por tanto:

$$
\sigma=\pi-\delta
$$

Si la profundidad radial de corte se designa como $a_{e}$, la distancia $l$ vendrá dada por la siguiente expresión, de acuerdo al esquema de la Figura 4:

$$
l=\frac{D}{2} \cdot \arccos \left(1-\frac{2 a_{e}}{D}\right)
$$

Se indican a continuación los ángulos que cubren las distintas etapas y el tipo de fase al que corresponde de acuerdo a la clasificación realizada en el capítulo 3.

i) $\left[0, \Phi_{2}-\sigma\right]$, fase $\mathrm{A}$ en diente $1 \mathrm{y}$ fase $\mathrm{C}$ en diente 2 
ii) $\left[\Phi_{2}-\sigma, \Phi_{2}\right]$, fase A

iii) $\left[\Phi_{2}, \delta\right]$, fase $\mathrm{B}$

iv) $[\delta, \pi]$, fase $\mathrm{C}$

Introduciendo estos datos en la expresión (6.24) se obtiene:

$$
\bar{F}=\frac{1}{\pi} \cdot\left[\int_{0}^{\Phi_{2}-\sigma}\left(F_{A 1}+F_{C 2}\right) \cdot d \alpha+\int_{\Phi_{2}-\sigma}^{\Phi_{2}} F_{A} \cdot d \alpha+\int_{\Phi_{2}}^{\delta} F_{B} \cdot d \alpha+\int_{\delta}^{\pi} F_{C} \cdot d \alpha\right]
$$

Dado que los dientes 1 y 2 están separados $180^{\circ}$ el primer término se puede expresar en función del ángulo de posición $\alpha$ :

$$
\begin{aligned}
& \bar{F}=\frac{1}{\pi} \cdot \int_{0}^{\Phi_{2}-\sigma}\left[F_{A}(\alpha)+F_{C}(\alpha+\pi)\right] \cdot d \alpha+\frac{1}{\pi} \cdot \int_{\Phi_{2}-\sigma}^{\Phi_{2}} F_{A} \cdot d \alpha+ \\
& \frac{1}{\pi} \cdot \int_{\Phi_{2}}^{\delta} F_{B} \cdot d \alpha+\frac{1}{\pi} \cdot \int_{\delta}^{\pi} F_{C} \cdot d \alpha
\end{aligned}
$$

Agrupando integrales se obtiene:

$$
\bar{F}=\frac{1}{\pi} \cdot\left(\int_{0}^{\Phi_{2}} F_{A} \cdot d \alpha+\int_{\Phi_{2}}^{\delta} F_{B} \cdot d \alpha+\int_{\delta}^{\Phi_{2}-\sigma+\pi} F_{C} \cdot d \alpha\right)
$$

Particularizando para cada dirección se obtiene:

$$
\begin{aligned}
& \overline{F_{x}}=\frac{1}{\pi} \cdot\left(\int_{0}^{\Phi_{2}} F_{x A} \cdot d \alpha+\int_{\Phi_{2}}^{\delta} F_{x B} \cdot d \alpha+\int_{\delta}^{\Phi_{2}+\delta} F_{x C} \cdot d \alpha\right) \\
& \overline{F_{y}}=\frac{1}{\pi} \cdot\left(\int_{0}^{\Phi_{2}} F_{y A} \cdot d \alpha+\int_{\Phi_{2}}^{\delta} F_{y B} \cdot d \alpha+\int_{\delta}^{\Phi_{2}+\delta} F_{y C} \cdot d \alpha\right)
\end{aligned}
$$

Sustituyendo en estas expresiones las fórmulas de las fuerzas en sus diferentes fases dadas en el capítulo 3 se obtienen las expresiones completas de la fuerza promedio en cada dirección según la modalidad de fresado. Para ello supondremos fresado tipo II, ya que es el caso más habitual en estos ensayos dado que la profundidad axial de corte es un valor elevado con respecto a la profundidad radial: 


\subsubsection{Fresado en oposición}

Aplicando el modelo de fresado ideal a las expresiones anteriores y realizando las operaciones se obtienen las fuerzas medias:

$$
\begin{gathered}
\overline{F_{x}}=\frac{\delta}{\pi} \cdot \frac{D}{2 \cdot \tan \beta} \cdot\left[\begin{array}{l}
K_{t e} \cdot \sin \Phi_{2}-K_{r e} \cdot \cos \Phi_{2}+K_{r e}+ \\
\frac{f_{z}}{4} \cdot\left(-K_{t c} \cdot \cos 2 \Phi_{2}+K_{t c}+2 K_{r c} \cdot \Phi_{2}-K_{r c} \cdot \sin 2 \Phi_{2}\right)
\end{array}\right] \\
\overline{F_{y}}=\frac{\delta}{\pi} \cdot \frac{D}{2 \cdot \tan \beta} \cdot\left[\begin{array}{l}
K_{t e} \cdot \cos \Phi_{2}-K_{t e}+K_{r e} \cdot \sin \Phi_{2}- \\
\frac{f_{z}}{4} \cdot\left(2 K_{t c} \cdot \Phi_{2}-K_{t c} \cdot \sin 2 \Phi_{2}-K_{r c} \cdot \cos 2 \Phi_{2}+K_{r c}\right)
\end{array}\right]
\end{gathered}
$$

Se puede observar que estas expresiones coinciden con las de la fase B del fresado tipo II multiplicadas por el factor $\delta / \pi$. Esto es consistente con el hecho de que entre el corte de dos dientes consecutivos existe una distancia angular $\pi-\delta$ y por tanto, la fracción de periodo de corte frente al caso de fuerza constante es precisamente $\delta / \pi$. Si las condiciones fueran de fuerza constante el parámetro $\delta$ coincidiría $\pi$ y las expresiones coincidirían con las que se dedujeron para ese tipo de corte.

De forma análoga a como se hizo entonces, se pueden identificar las fuerzas de filo y de corte utilizando las expresiones (6.2) y (6.3):

$$
\begin{gathered}
\overline{F_{x e}}=\frac{\delta}{\pi} \cdot \frac{D}{2 \cdot \tan \beta} \cdot\left(K_{t e} \cdot \sin \Phi_{2}-K_{r e} \cdot \cos \Phi_{2}+K_{r e}\right) \\
\overline{F_{y e}}=\frac{\delta}{\pi} \cdot \frac{D}{2 \cdot \tan \beta} \cdot\left(K_{t e} \cdot \cos \Phi_{2}-K_{t e}+K_{r e} \cdot \sin \Phi_{2}\right) \\
\overline{F_{x c}}=\frac{\delta}{\pi} \cdot \frac{D}{8 \cdot \tan \beta} \cdot\left(-K_{t c} \cdot \cos 2 \Phi_{2}+K_{t c}+2 K_{r c} \cdot \Phi_{2}-K_{r c} \cdot \sin 2 \Phi_{2}\right) \\
\overline{F_{y c}}=\frac{\delta}{\pi} \cdot \frac{D}{8 \cdot \tan \beta} \cdot\left(2 K_{t c} \cdot \Phi_{2}-K_{t c} \cdot \sin 2 \Phi_{2}-K_{r c} \cdot \cos 2 \Phi_{2}+K_{r c}\right)
\end{gathered}
$$


De aquí se pueden obtener los coeficientes de las fuerzas en función de las fuerzas promedio, como se hizo para el fresado con fuerza constante:

$$
\begin{gathered}
K_{t e}=\frac{\pi}{\delta} \cdot \frac{\tan \beta}{D} \cdot \frac{\overline{F_{x e}} \cdot \sin \Phi_{2}+\overline{F_{y e}} \cdot\left(\cos \Phi_{2}-1\right)}{1-\cos \Phi_{2}} \\
K_{r e}=\frac{K_{t e} \cdot \sin \Phi_{2}-\overline{F_{x e}} \cdot \frac{\pi}{\delta} \cdot \frac{2 \cdot \tan \beta}{D}}{\cos \Phi_{2}-1} \\
K_{r c} \frac{\pi}{\delta} \cdot \frac{8 \cdot \tan \beta}{D} \cdot \frac{\overline{F_{x c}} \cdot\left(2 \Phi_{2}-\sin 2 \Phi_{2}\right)+\overline{F_{y c}} \cdot\left(\cos 2 \Phi_{2}-1\right)}{2+4 \Phi_{2}{ }^{2}-4 \Phi_{2} \cdot \sin 2 \Phi_{2}-2 \cos 2 \Phi_{2}} \\
K_{t c}=\frac{K_{r c} \cdot\left(2 \Phi_{2}-\sin 2 \Phi_{2}\right)+\overline{F_{x c}} \cdot \frac{\pi}{\delta} \cdot \frac{8 \cdot \tan \beta}{D}}{1-\cos 2 \Phi_{2}}
\end{gathered}
$$

\subsubsection{Fresado en concordancia}

De la misma manera que en el apartado anterior se aplican las fuerzas del modelo ideal a las expresiones (7.33) y (7.34) para obtener las fuerzas medias:

$$
\begin{gathered}
\overline{F_{x}}=\frac{\delta}{\pi} \cdot \frac{D}{2 \cdot \tan \beta} \cdot\left[\begin{array}{l}
-K_{t e} \cdot \cos \Phi_{2}+K_{t e}-K_{r e} \cdot \sin \Phi_{2}+ \\
\frac{f_{z}}{4} \cdot\left(-K_{t c} \cdot \cos 2 \Phi_{2}+K_{t c}-2 K_{r c} \cdot \Phi_{2}-K_{r c} \cdot \sin 2 \Phi_{2}\right)
\end{array}\right] \\
\overline{F_{y}}=\frac{\delta}{\pi} \cdot \frac{D}{2 \cdot \tan \beta} \cdot\left[\begin{array}{l}
K_{t e} \cdot \sin \Phi_{2}-K_{r e} \cdot \cos \Phi_{2}+K_{r e}+ \\
\frac{f_{z}}{4} \cdot\left(2 K_{t c} \cdot \Phi_{2}+K_{t c} \cdot \sin 2 \Phi_{2}-K_{r c} \cdot \cos 2 \Phi_{2}+K_{r c}\right)
\end{array}\right]
\end{gathered}
$$

Identificando las fuerzas del filo y las de corte se obtiene: 


$$
\begin{gathered}
\overline{F_{x e}}=\frac{\delta}{\pi} \cdot \frac{D}{2 \cdot \tan \beta} \cdot\left(-K_{t e} \cdot \cos \Phi_{2}+K_{t e}-K_{r e} \cdot \sin \Phi_{2}\right) \\
\overline{F_{y e}}=\frac{\delta}{\pi} \cdot \frac{D}{2 \cdot \tan \beta} \cdot\left(K_{t e} \cdot \sin \Phi_{2}-K_{r e} \cdot \cos \Phi_{2}+K_{r e}\right) \\
\overline{F_{x c}}=\frac{\delta}{\pi} \cdot \frac{D}{8 \cdot \tan \beta} \cdot\left(-K_{t c} \cdot \cos 2 \Phi_{2}+K_{t c}-2 K_{r c} \cdot \Phi_{2}-K_{r c} \cdot \sin 2 \Phi_{2}\right) \\
\overline{F_{y c}}=\frac{\delta}{\pi} \cdot \frac{D}{8 \cdot \tan \beta} \cdot\left(2 K_{t c} \cdot \Phi_{2}+K_{t c} \cdot \sin 2 \Phi_{2}-K_{r c} \cdot \cos 2 \Phi_{2}+K_{r c}\right)
\end{gathered}
$$

De estas expresiones se pueden obtener los coeficientes de fuerza:

$$
\begin{gathered}
K_{r e}=-\frac{\pi}{\delta} \cdot \frac{\tan \beta}{D} \cdot \frac{\overline{F_{x e}} \cdot \sin \Phi_{2}+\overline{F_{y e}} \cdot\left(\cos \Phi_{2}-1\right)}{1-\cos \Phi_{2}} \\
K_{t e}=-\frac{\overline{F_{x e}} \cdot \frac{\pi}{\delta} \cdot \frac{2 \cdot \tan \beta}{D}+K_{r e} \cdot \sin \Phi_{2}}{\cos \Phi_{2}-1} \\
K_{r c}=-\frac{\pi}{\delta} \cdot \frac{4 \cdot \tan \beta}{D} \cdot \frac{\overline{F_{x c}} \cdot\left(2 \Phi_{2}+\sin 2 \Phi_{2}\right)+\overline{F_{y c}} \cdot\left(\cos 2 \Phi_{2}-1\right)}{1-\cos 2 \Phi_{2}+2 \Phi_{2}{ }^{2}+2 \Phi_{2} \cdot \sin 2 \Phi_{2}} \\
K_{t c}=-\frac{\frac{\pi}{F_{x c}} \cdot \frac{\pi}{\delta} \cdot \frac{8 \cdot \tan \beta}{D}+K_{r c} \cdot\left(2 \Phi_{2}+\sin 2 \Phi_{2}\right)}{\cos 2 \Phi_{2}-1}
\end{gathered}
$$

\subsection{Medición de la desviación excéntrica de herramienta}

La desviación excéntrica de la herramienta viene determinada por dos parámetros: la magnitud de la desviación $\rho$ y su ángulo $\lambda$, según se indica en el capítulo 3 al establecer el modelo de desviación excéntrica. 
El parámetro $\rho$ puede ser medido directamente mediante un reloj comparador en contacto con la herramienta mientras ésta es girada manualmente. Para ello se registrará la diferencia entre las dos medidas extremas del reloj y este valor constituirá la distancia máxima entre las posiciones extremas de la herramienta. Dicha medida proporcionará el doble del parámetro $\rho$, según se deduce del modelo adoptado. Esta expresión establece un ángulo $\lambda$ entre la posición de la desviación máxima y la del filo 1. Como las mediciones realizadas por el reloj comparador se realizan sobre toda el perímetro del mango, el ángulo $\lambda$ irá variando entre 0 y $2 \pi$ y el radio tendrá, según la expresión (3.39), los siguientes valores extremos:

$$
\begin{aligned}
& r_{\max }=\frac{D}{2}+\rho \\
& r_{\min }=\frac{D}{2}-\rho
\end{aligned}
$$

La medida del reloj comparador será la diferencia entre ambos valores y por tanto:

$$
\rho=\frac{\Delta r}{2}
$$

El ángulo $\lambda$ deberá ser medido mediante una escala angular graduada situada en el cabezal de la máquina, sobre la cual se registrará el ángulo que es preciso girar la herramienta para que pase de la posición de alineación entre la punta del reloj comparador y el filo 1 a la posición de máxima desviación de la herramienta.

\subsection{Medición del radio de filo de la herramienta}

El radio de filo de la herramienta es un parámetro necesario para caracterizar el efecto tamaño, según se indicó en los capítulos 4 y 5 . Este valor establecerá el comienzo de corte del filo, según la expresión (4.46).

Para determinar su valor es preciso tomar imágenes ampliadas de la punta de las fresas y tomar medidas sobre el perfil del filo para obtener el radio mediante estudio 
geométrico. En las figura 6.2 y 6.3 se muestra imágenes de fresas DIXI de $0.6 \mathrm{~mm}$ de diámetro con distinto radio de filo debido al desgaste.

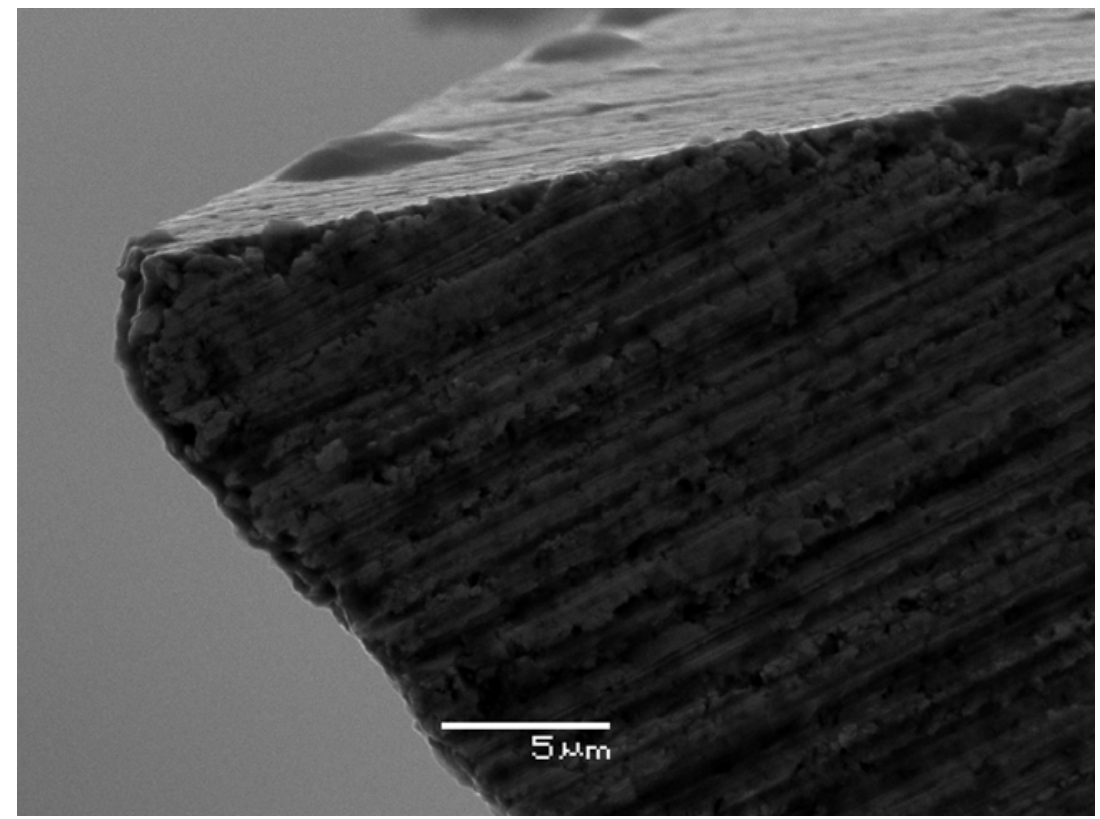

Figura 6.2. Imagen del filo de una fresa DIXI de $0.6 \mathrm{~mm}$ de diámetro nueva

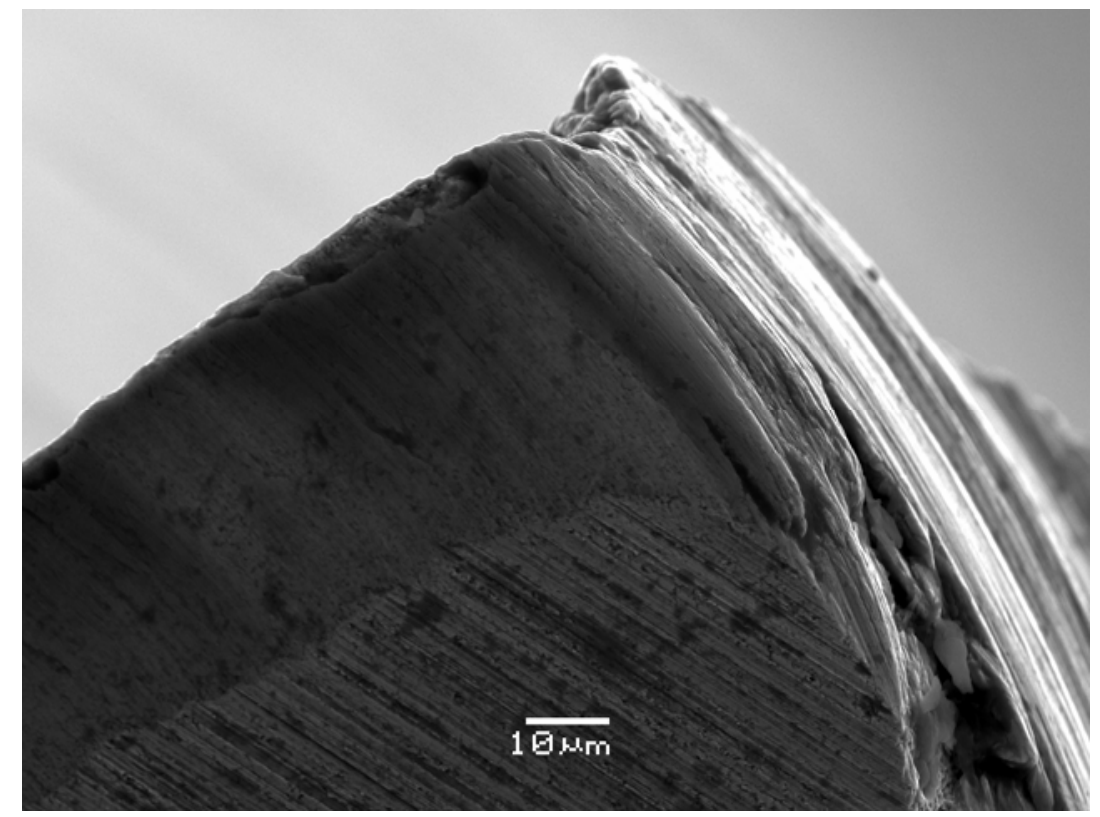

Figura 6.3. Imagen del filo de una fresa DIXI de $0.6 \mathrm{~mm}$ de diámetro desgastada

En las figuras 6.4 y 6.5 se muestran imágenes de fresas Sandvik de $0.6 \mathrm{~mm}$ de diámetro con distintos radios de filo debido al diferente desgaste. 


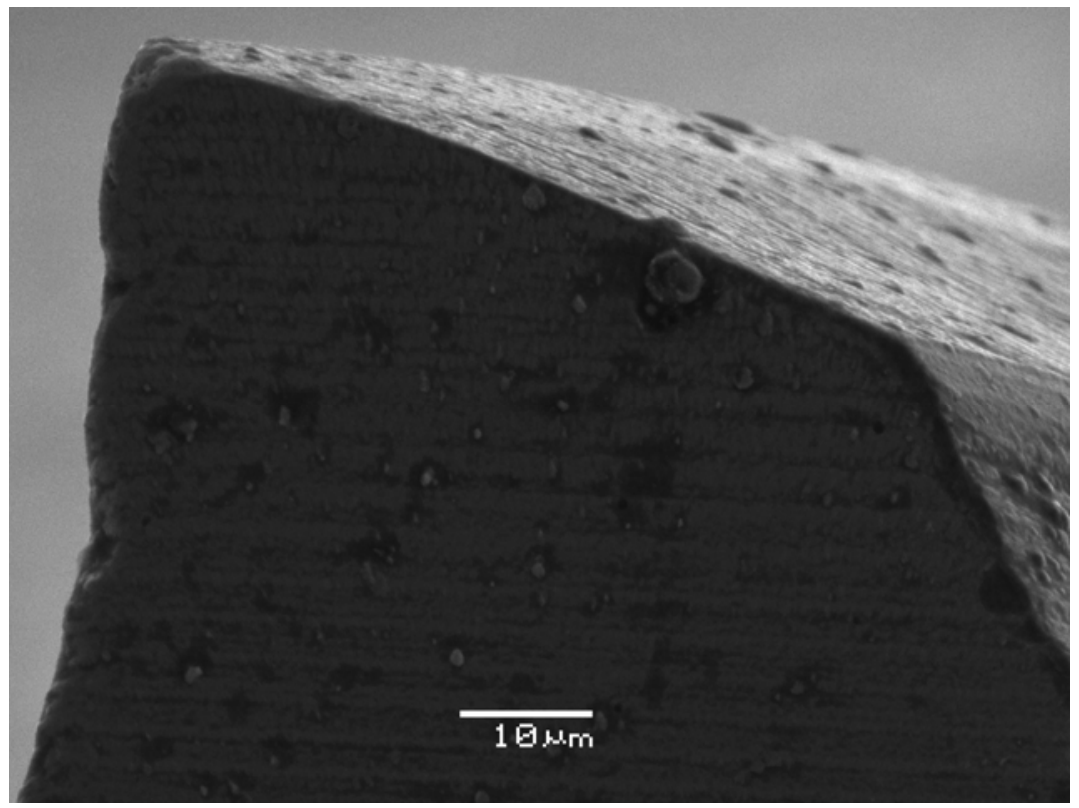

Figura 6.4. Imagen del filo de una fresa Sandvik de $0.6 \mathrm{~mm}$ de diámetro nueva

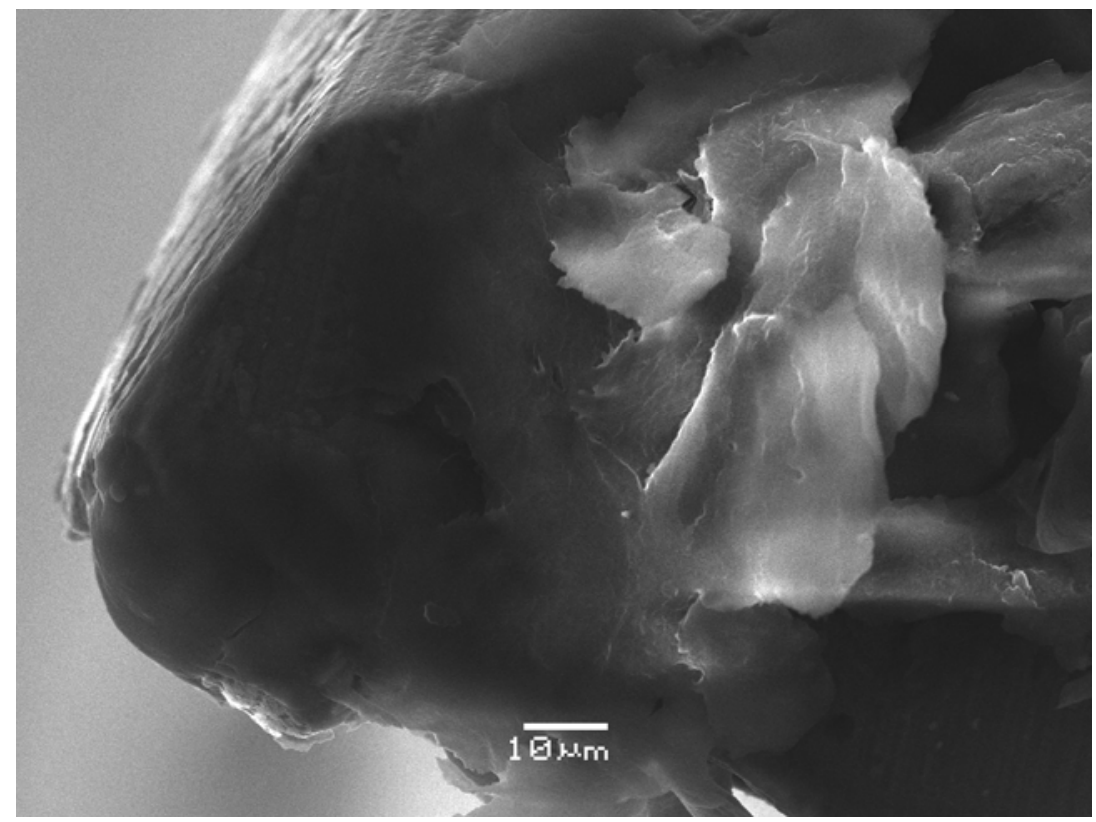

Figura 6.5. Imagen del filo de una fresa Sandvik de $0.6 \mathrm{~mm}$ de diámetro desgastada

Se puede observar que el redondeo del filo es muy superior en las fresas desgastadas que en las nuevas, por lo que habrá que tener en cuenta esta circunstancia a la hora de realizar la predicción de las fuerzas mediante el modelo. Se requerirá utilizar un radio de filo en el modelo adaptado al grado de desgaste que tengan las fresas. 
Sobre estas imágenes se pueden tomar medidas mediante la librería de herramientas para imágenes del programa MATLAB. Esta herramienta mide número de píxeles entre dos puntos. Estas medidas se pueden comparar con la referencia de distancia indicada en la imagen para obtener la medida real. Las medidas consistirán en 3 puntos tomados sobre el perfil del filo, que constituirán un triángulo, cuyos lados se denominan $u, v \mathrm{y} w$, según se indica en la figura 6.6.

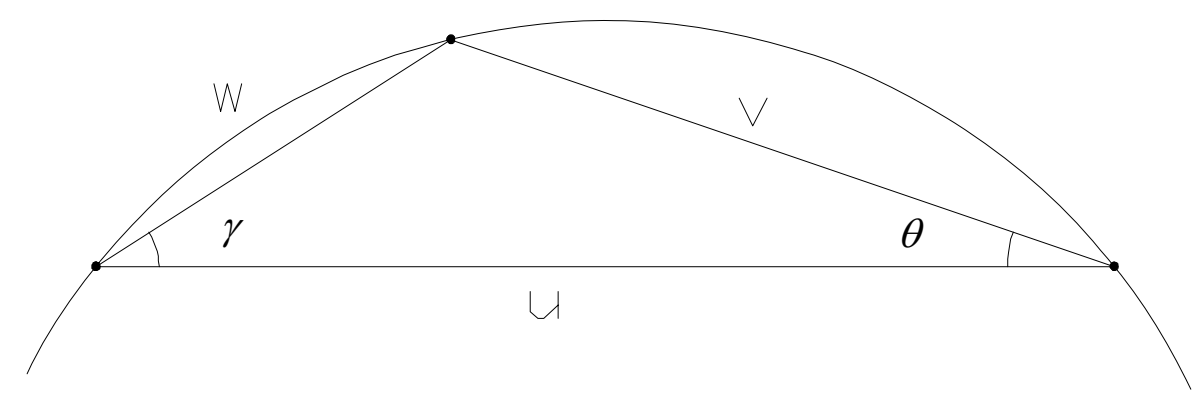

Figura 6.6. Esquema de los puntos tomados sobre el perfil del filo

En función de dichas distancias y de los ángulos $\gamma$ y $\theta$ indicados en la figura 7.6 se deduce el radio de la circunferencia que pasa por los tres puntos del filo, que coincidirá con el radio del filo:

$$
r_{\varepsilon}=\sqrt{\left(\frac{2 u-v \cdot \cos \theta-w \cdot \cos \gamma}{2 \sin \gamma+2 \cdot \cos \gamma \cdot \tan \theta}\right)^{2}+\left(\frac{w}{2}\right)^{2}}
$$

\subsection{Medición de la rigidez de la herramienta}

La constante $M$ puede determinarse experimentalmente para cada herramienta usada en el proceso. De acuerdo a la expresión (3.58) esta constante depende de la longitud de herramienta que sobresale del husillo, del material y la forma de la herramienta y de la distancia de la punta a la resultante de las fuerzas, que depende a su vez de la profundidad axial de corte. Por tanto, para cada combinación de condiciones del proceso existirá un valor distinto de $M$. 
El método para determinar el valor de $M$ consiste en situar la herramienta junto a la pieza de tal manera que la punta esté por debajo de la superficie de la pieza una distancia igual a la mitad de la profundidad axial de corte del ensayo al que se refiera la medición. De esta manera, se podrá determinar la relación entre fuerza y desplazamiento según la expresión (3.59), ya que la fuerza distribuida correspondiente al corte puede suponerse concentrada en la mitad de la profundidad de corte.

Después la herramienta se desplaza por medio del control CNC de la máquina hasta que toque la pieza. A partir de este punto se seguirá desplazando la herramienta de tal manera que se doblará bajo la acción de la fuerza que el borde de la pieza ejerce sobre ella. En el dinamómetro se detectará una fuerza que será registrada junto con el desplazamiento medido por el control $\mathrm{CNC}$ en varias posiciones. Dado que la fuerza será proporcional al desplazamiento, estos valores formarán una línea recta al ser representados en una gráfica. Debido a que los valores no estarán perfectamente alineados debido a errores en las medidas, será preciso aplicar la técnica de la regresión lineal para obtener la relación.

En la tabla 6.1 se indican los valores de fuerza y desplazamiento tomados para una fresa de $0.4 \mathrm{~mm}$ de diámetro. La Figura 10 muestra la gráfica que representa dichos valores, junto con su recta de regresión. El coeficiente de correlación de esta regresión fue 0.9998, lo cual indica el buen ajuste que tienen los datos respecto a su modelo teórico. La constante de proporcionalidad que aporta la regresión es 83.9 , que será el valor de $M$ para este caso concreto.

\begin{tabular}{|c|c|}
\hline Coordenada $\boldsymbol{x}(\mathbf{m m})$ & Fuerza en $\boldsymbol{x} \mathbf{( N )}$ \\
\hline$-0,0800$ & 1,22 \\
\hline$-0,0700$ & 2,14 \\
\hline$-0,0600$ & 2,98 \\
\hline$-0,0500$ & 3,77 \\
\hline$-0,0400$ & 4,62 \\
\hline$-0,0300$ & 5,45 \\
\hline
\end{tabular}

Tabla 6.1. Desplazamientos y fuerzas en el ensayo de deflexión de herramienta 
En la figura 6.7 se muestran los valores experimentales de la fuerza medida por el dinamómetro frente a los valores del desplazamiento establecido por el control numérico de la máquina. Se puede apreciar que son valores alineados y que, por tanto, se verifica el carácter proporcional de la deflexión de la herramienta con respecto a la fuerza aplicada.

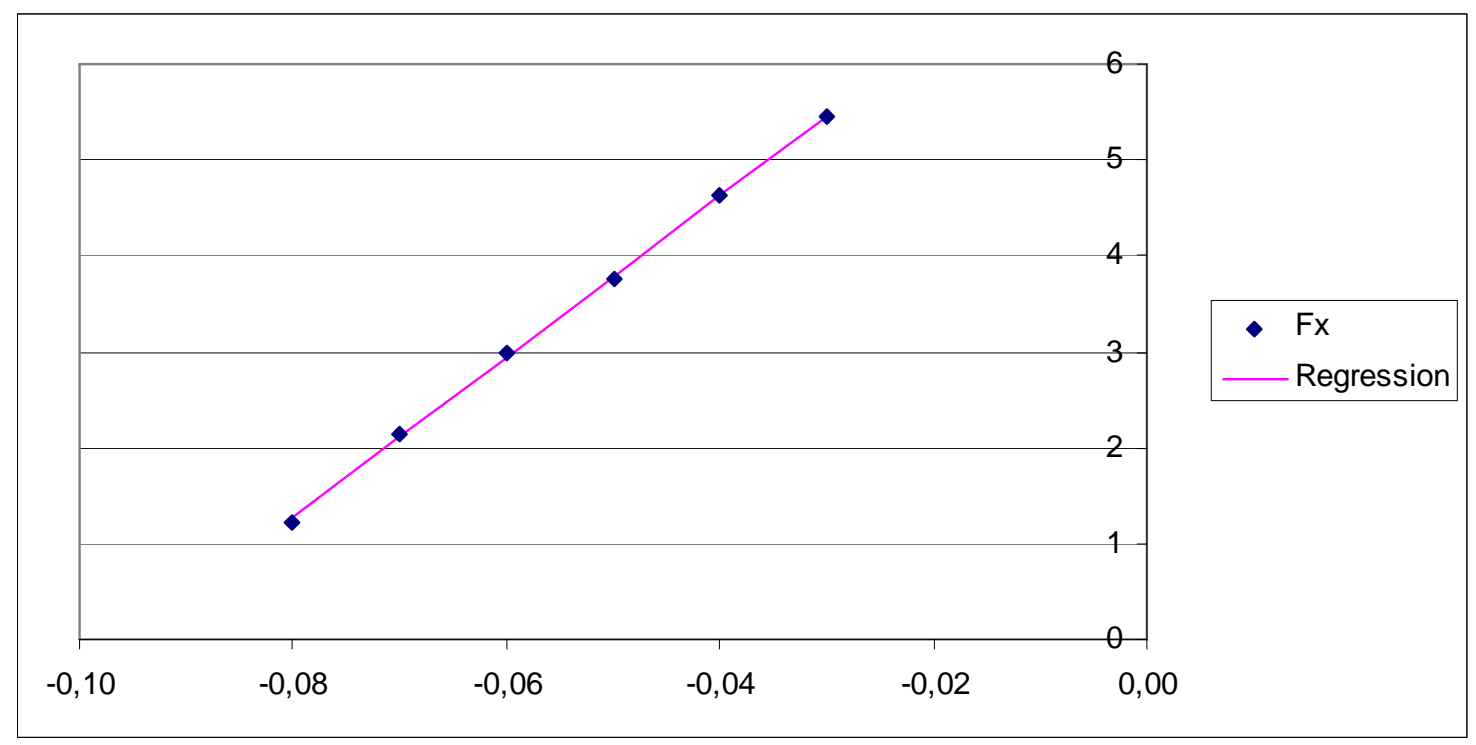

Figura 6.7. Gráfica Fuerza-Desplazamiento en el ensayo de deflexión de herramienta 
ANÁLISIS DE LAS VIBRACIONES DEL SISTEMA

\subsection{Determinación de la frecuencia natural del sistema}

Como se explicó en el capítulo 2 la dinámica del sistema se puede esquematizar de modo sencillo mediante una masa, un resorte elástico y un amortiguador viscoso. Este sistema tendrá una frecuencia natural de vibración que será aquélla a la que oscile el sistema cuando se le separe de su posición de equilibrio y se le deje evolucionar libremente. Para medir experimentalmente esta frecuencia se seguirá el procedimiento de golpear la pieza de trabajo y observar las oscilaciones posteriores. Se golpeará en la cara lateral para medir las oscilaciones en el eje $x$ y en la cara anterior para medirlas en el eje $y$.

Dado que las aceleraciones de la pieza oscilante se reflejarán en forma de fuerzas en la sujeción se podrá medir fácilmente la frecuencia de vibración de la pieza de trabajo observando las oscilaciones que experimenta la fuerza del dinamómetro al que está sujeta. Esta frecuencia natural será la que determine las condiciones de retemblado, que tendrán lugar cuando se someta al sistema a vibraciones forzadas a esa frecuencia.

En el proceso se utilizarán dos configuraciones distintas de la máquina dado que para cada rango de velocidad se empleará un eje giratorio distinto. Dado que el rango general de trabajo es 20000 - 120000 r.p.m. se deberá usar el eje de velocidad intermedia (6000 - 40000 r.p.m.) y el rápido (80000 - 160000 r.p.m.). Por tanto, será necesario medir las frecuencias naturales del sistema con cada eje.

En las figuras 7.1 y 7.2 se muestra la oscilación en las direcciones $x$ e $y$ que con el eje rápido experimenta el dinamómetro al que está sujeta la pieza cuando ésta es golpeada. El material de la pieza es aluminio 7075. 


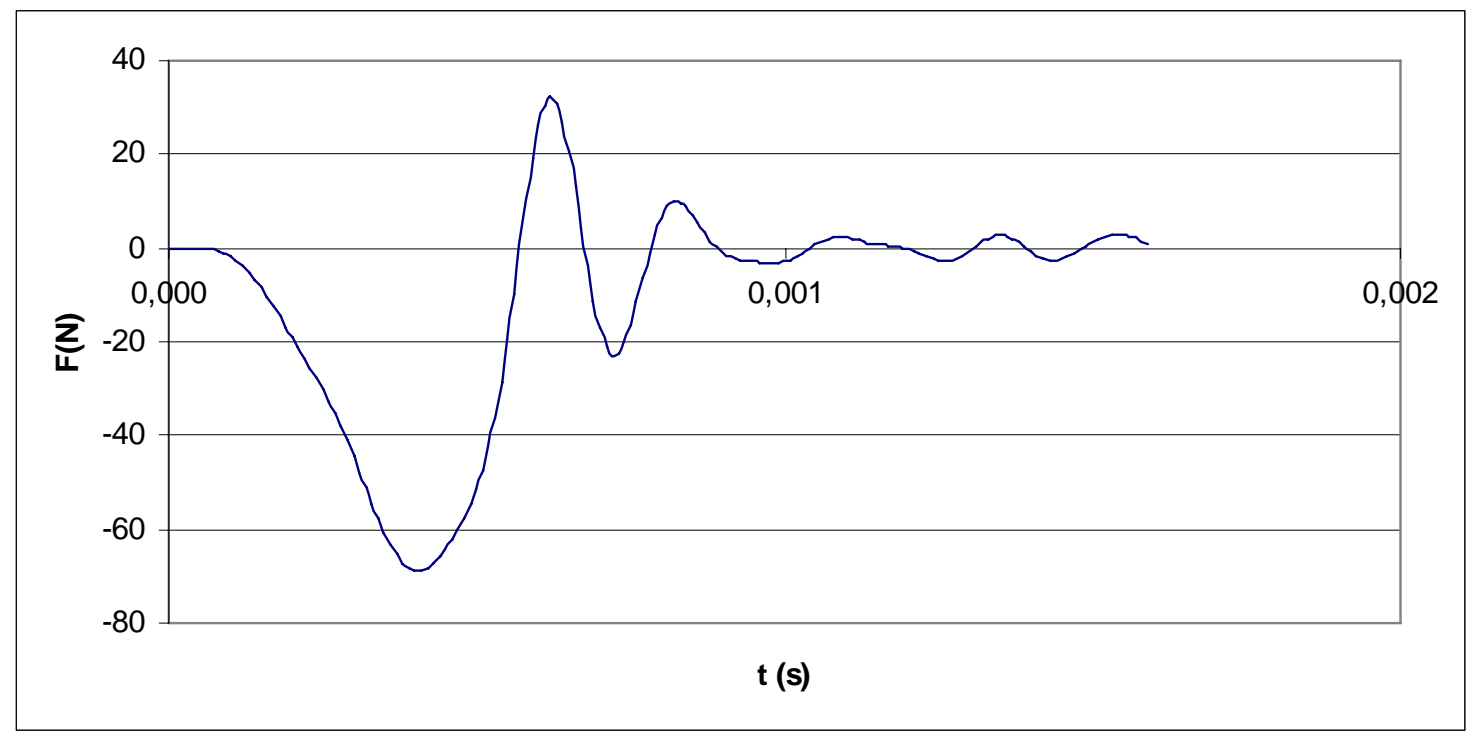

Figura 7.1. Fuerza en $x$ en oscilación libre con el eje rápido

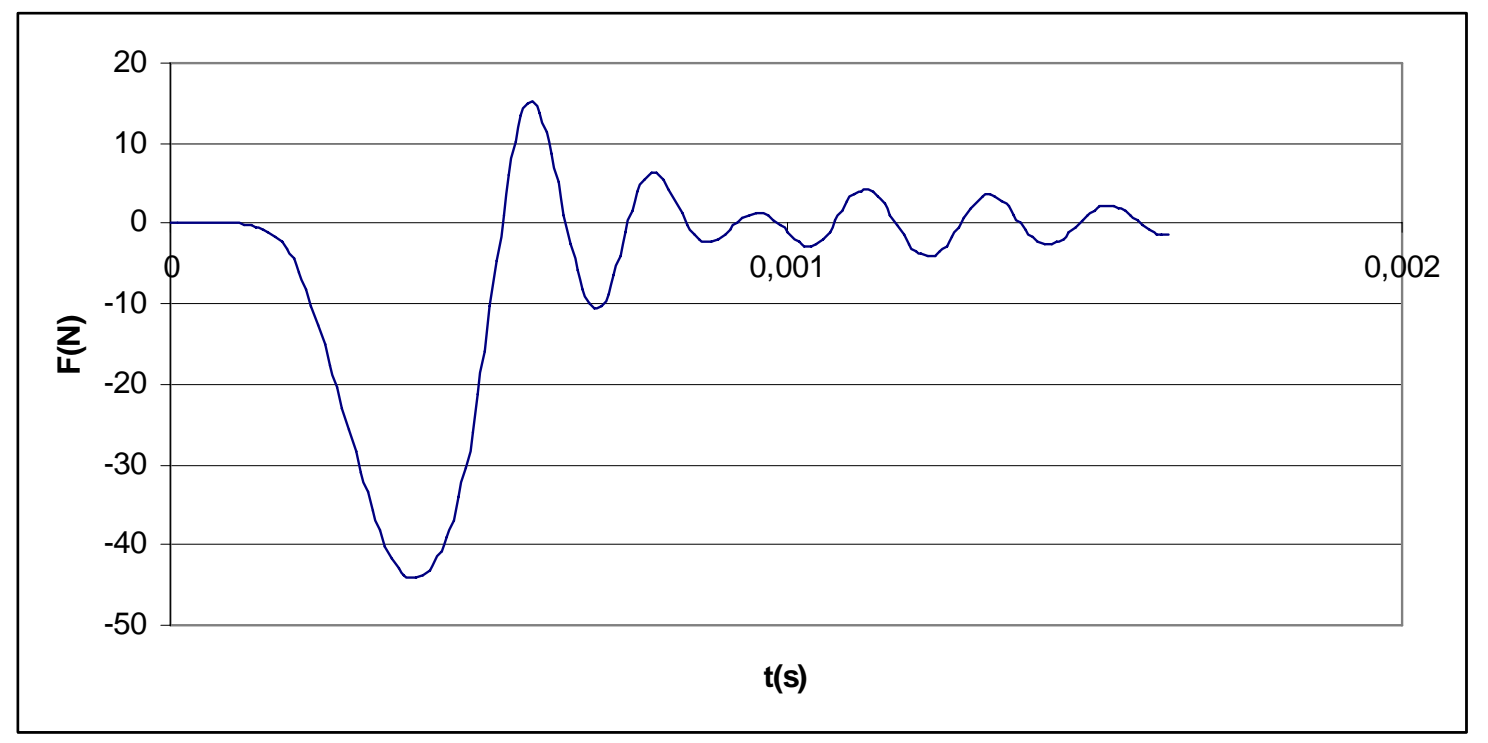

Figura 7.2. Fuerza en y en oscilación libre con el eje rápido

En estas gráficas se observa una señal de vibración amortiguada que no es totalmente senoidal, por lo que se puede deducir que el sistema es multimodal y tiene varias frecuencias naturales de vibración. Se determinará simplemente una de ellas para hacer el ensayo de retemblado. Midiendo la distancia entre los dos primeros picos de ambas gráficas se puede determinar la frecuencia natural más baja, que resulta:

$$
f_{x}=3125 \mathrm{~Hz}
$$




$$
f_{y}=3448 \mathrm{~Hz}
$$

Estas frecuencias son relativamente altas, dado que la frecuencia habitual de giro del eje es sensiblemente inferior a estos valores. No obstante, dado que la fuerza de fresado es una señal oscilatoria no senoidal, su transformada de Fourier presentará picos a frecuencias muy superiores a la fundamental. Si la frecuencia de alguno de estos picos coincide con la frecuencia natural de la pieza en alguno de sus ejes puede ocurrir el fenómeno de la resonancia, que generará unas vibraciones de magnitud elevada que se superponen a la señal del proceso.

En las figuras 7.3 y 7.4 se muestran los resultados de vibraciones libres del sistema con el eje de velocidad intermedia. En ellas se observa más claramente que en el caso anterior que la vibración natural está compuesta por señales de diferentes frecuencias, dado que el sistema es multimodal [9]. Si se observa la distancia entre los dos primeros picos se determina que las frecuencias naturales son:

$$
\begin{aligned}
& f_{x}=3846 \mathrm{~Hz} \\
& f_{y}=4545 \mathrm{~Hz}
\end{aligned}
$$

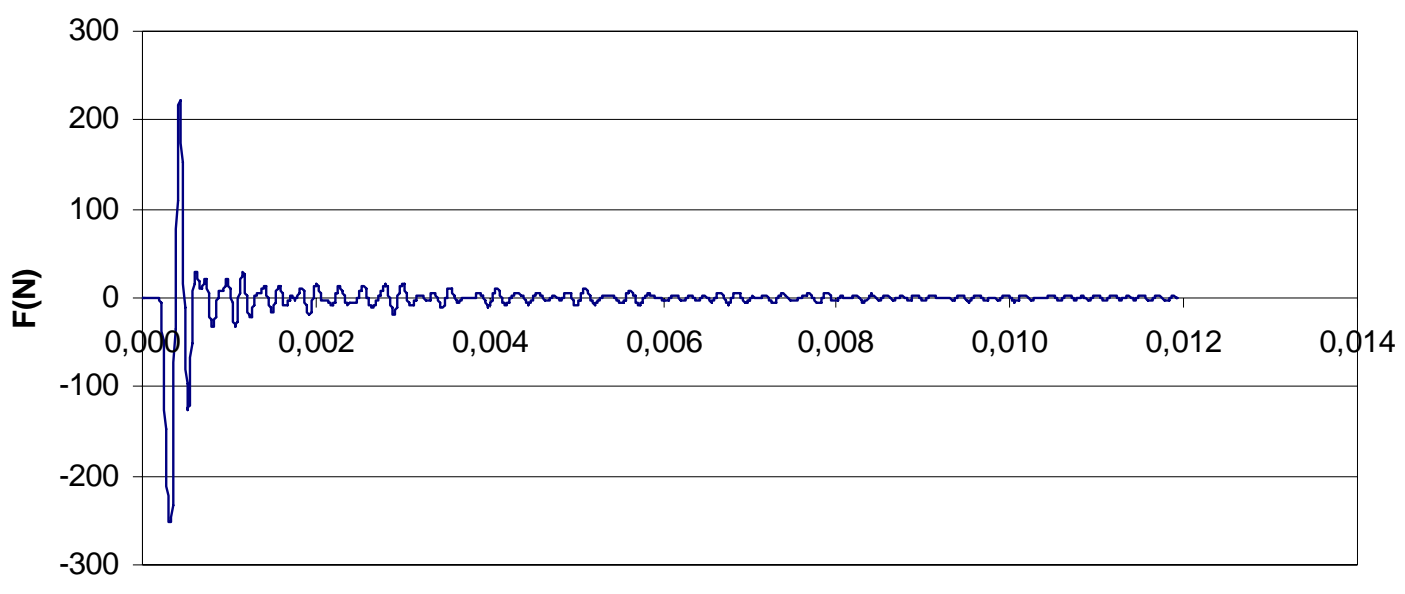

$\mathrm{t}(\mathbf{s})$

Figura 7.3. Fuerza en $x$ en oscilación libre con el eje intermedio 


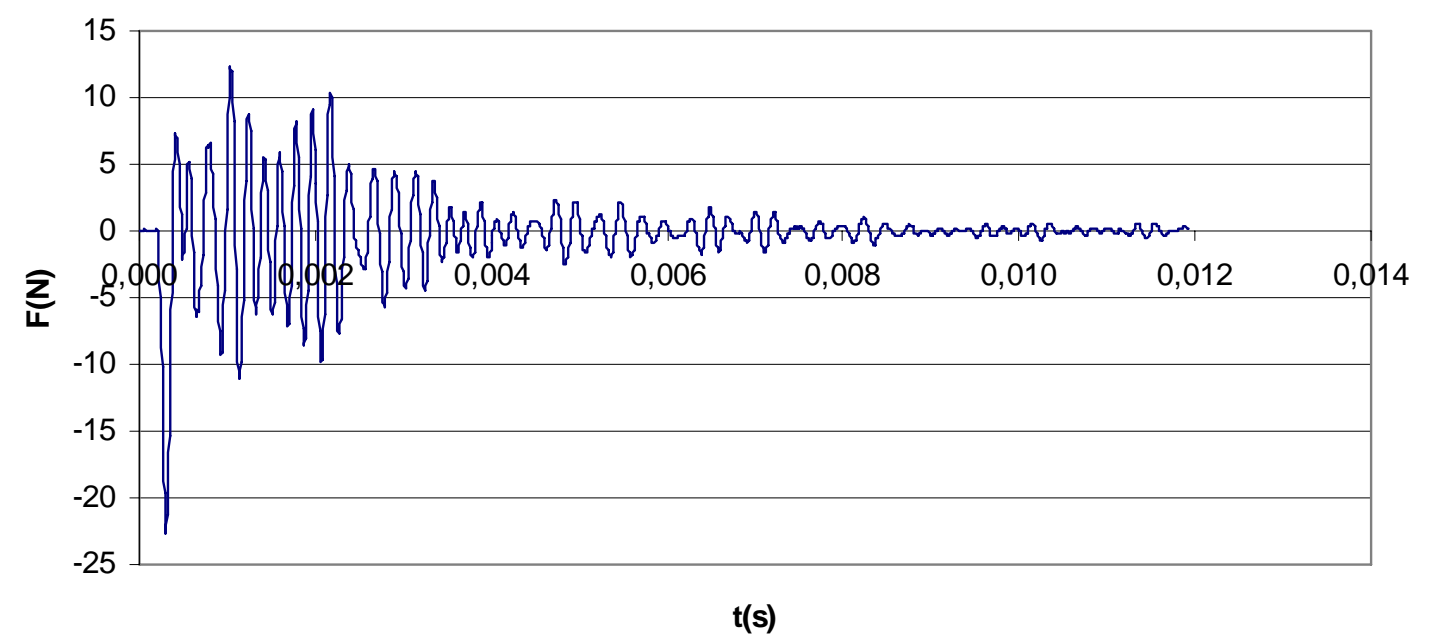

Figura 7.4. Fuerza en y en oscilación libre con el eje intermedio

Sin embargo, si se mide la distancia entre picos cuando la señal ya está muy amortiguada (0.006 s aproximadamente) las frecuencias que se obtienen son las siguientes:

$$
\begin{aligned}
& f_{x}=5263 \mathrm{~Hz} \\
& f_{y}=5556 \mathrm{~Hz}
\end{aligned}
$$

Lo cual significa que los dos primeros valores corresponden a las frecuencias del primer modo de vibración y los dos siguientes a las del segundo modo.

\subsection{Trabajo en condiciones de retemblado}

De acuerdo con algunos estudios [42], el retemblado se dará cuando la frecuencia fundamental de la señal de fuerza sea igual a alguna de las frecuencias naturales de vibración del sistema. La frecuencia fundamental de la señal de fuerza corresponderá al doble de la frecuencia de giro del husillo, que será la de paso de los dientes. Por tanto:

$$
f_{r}=2 \cdot \frac{n_{r}}{60}
$$


En el caso de la pieza de aluminio antes mencionada, se pueden obtener las velocidades de giro críticas a partir de las frecuencias naturales de vibración determinadas anteriormente:

$$
\begin{aligned}
& n_{r 1}=93750 \text { r.p.m. } \\
& n_{r 2}=103440 \text { r.p.m. }
\end{aligned}
$$

Para comprobar la existencia de retemblado a estas velocidades se realizó un ensayo de fresado frontal con una fresa de $0.4 \mathrm{~mm}$ de diámetro con las siguientes condiciones: $n=$ 93750 r.p.m. (118 m/min), $a_{p}=0.15 \mathrm{~mm}, a_{e}=0.2 \mathrm{~mm}, f_{z}=0.003 \mathrm{~mm}$, fresado en oposición. La señal de fuerzas obtenida se muestra en la figura 7.5.

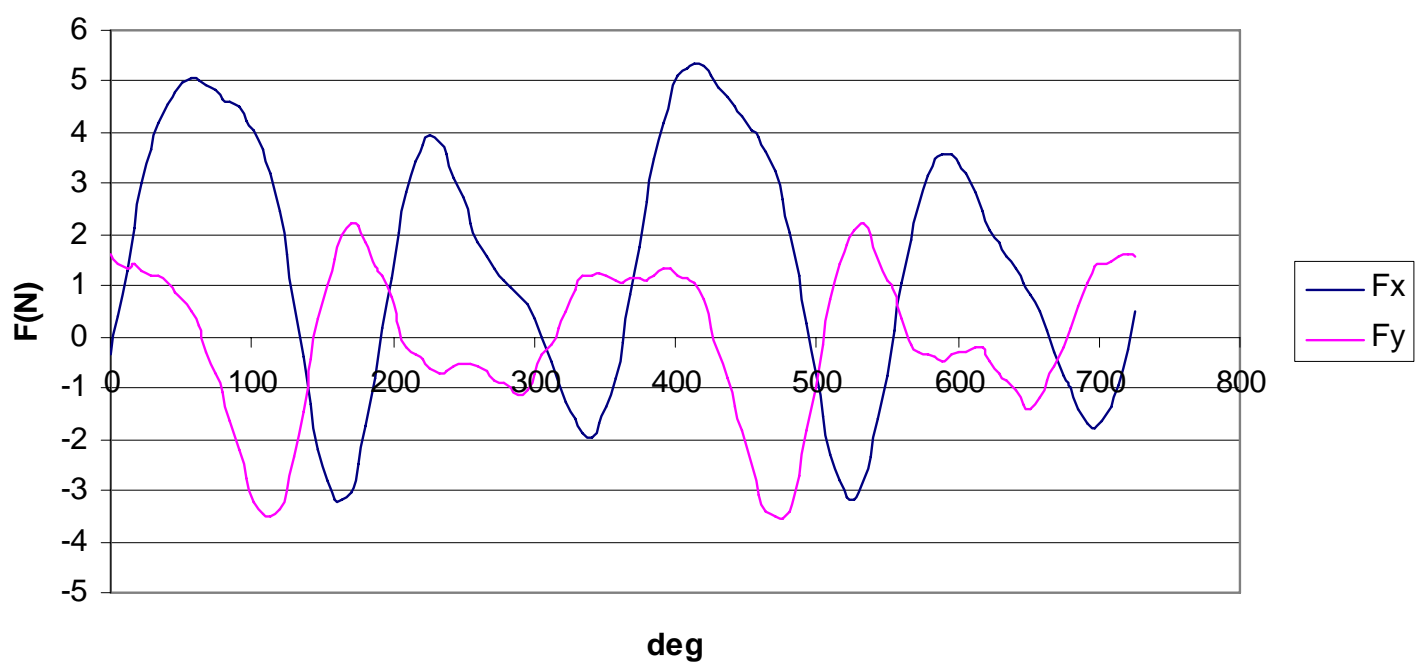

Figura 7.5. Fuerzas del proceso en condiciones de retemblado

Se puede observar en la figura que la señal de fuerzas difiere mucho de la evolución que cabría esperar. No hay periodos de pasos por 0 en ninguna de las dos señales cuando debería haberlos, dado que es un perfilado en el que la profundidad radial de corte corresponde con la mitad del diámetro de la fresa, por lo que cada filo debería estar cortando únicamente la mitad del tiempo. Por otro lado, no debería haber valores negativos de la fuerza en $x$ según el modelo propuesto, $y$ sin embargo aparecen con claridad periodos de fuerza negativa en esa dirección. Todo ello es debido a la vibración 
de la pieza amplificada por la acción de las fuerzas de corte en la misma frecuencia, que hace que se registre fuerza aun cuando la herramienta no está cortando.

En la figura 7.6 aparece el espectro de la señal de fuerza en el eje $x$. En él se ve claramente la predominancia del pico correspondiente a la frecuencia natural de vibración del sistema, que en este caso coincide con la frecuencia de paso de los dientes $(3125 \mathrm{~Hz})$. La vibración a la frecuencia de giro de la herramienta se corresponde con el primer pico que aparece, aproximadamente a $1500 \mathrm{~Hz}$. Este pico es debido al desequilibrio entre los dientes, dado que corresponde a una variación de la señal al ritmo de una vez por vuelta. Si no hubiese desviación excéntrica de la herramienta no aparecería este pico porque la señal que producen ambos dientes sería igual y únicamente habría repetición de valores al ritmo de paso de los dientes. Este desequilibrio se refleja en la diferencia de alturas de los picos correspondientes a distintos dientes en la gráfica de las fuerzas.

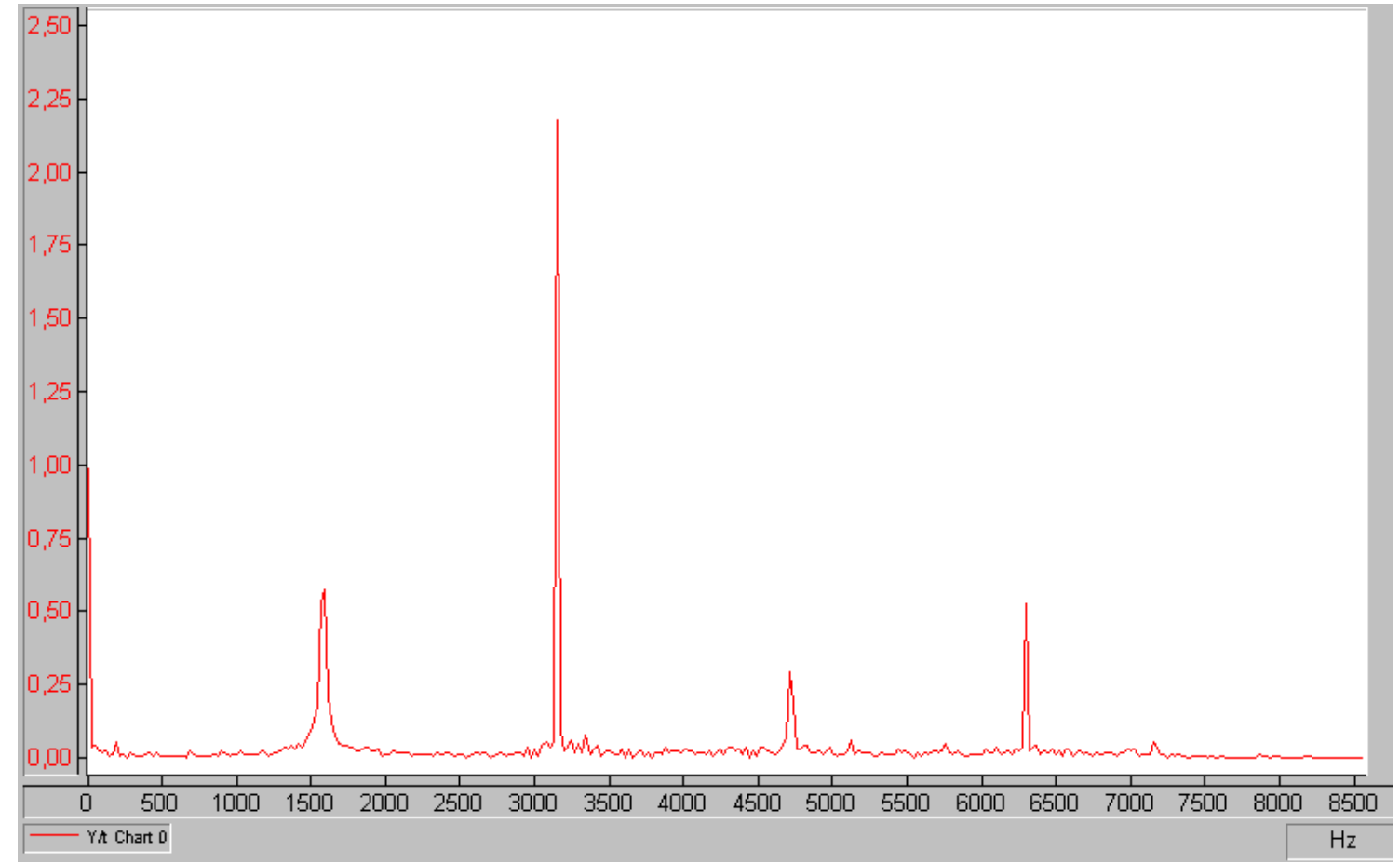

Figura 7.6. Transformada de Fourier de la señal de fuerza en el eje $x$

Se observan también los armónicos de la señal del primer pico a múltiplos enteros de dicha frecuencia. Aunque en general, esta señal y sus armónicos son de valor inferior a la señal principal, en este caso son todavía de menor cuantía relativa que en casos de no 
retemblado, debido a que la vibración a la frecuencia natural del sistema hace que se amplifique la señal a la frecuencia de paso de los dientes.

La distorsión que introduce este fenómeno del retemblado hace que las fuerzas no se ajusten al modelo y por tanto constituya un límite a la predicción de fuerzas del proceso mediante el modelo propuesto.

\subsection{Trabajo en condiciones de no retemblado}

Cuando se trabaja a una velocidad de giro distinta de los valores críticos determinados en el apartado anterior se obtiene una señal de fuerza no distorsionada completamente por el retemblado, sino sólo ligeramente afectada por la vibración natural del sistema, que se traduce en una oscilación de frecuencia superior superpuesta a la fuerza de corte en la gráfica de fuerzas. En el caso de no retemblado estas vibraciones se pueden eliminar mediante filtrado.

A continuación se presentan los resultados de un ensayo realizado a una frecuencia de giro notablemente inferior a la frecuencia de resonancia pero manteniendo el resto de condiciones usadas en el ensayo de retemblado. La velocidad de giro del ensayo fue de 23873 r.p.m., que corresponde a una velocidad de corte de $30 \mathrm{~m} / \mathrm{min}$. En la figura $7.7 \mathrm{se}$ muestran las señales de las fuerzas del proceso para este ensayo. Se puede observar que la señal se corresponde sustancialmente con el modelo propuesto, dado que existen pasos por 0 y los signos de las fuerzas se ajustan a los previstos por el modelo para el fresado en oposición, es decir, positivo para la fuerza en $x$ y alternante para la fuerza en y. Se aprecia también la influencia de la desviación excéntrica de la herramienta, que hace que los picos correspondientes a distintos dientes tengan distinta altura, lo cual permite predecir que en el espectro de la señal existirá un pico a la frecuencia de giro de la herramienta, es decir, a $398 \mathrm{~Hz}$.

Por otro lado, vemos que los valores extremos de las fuerzas son sustancialmente menores que los que aparecían en condiciones de retemblado, debido a que no existe la amplificación que se daba en esas condiciones. 
Se observa además una vibración de frecuencia superior superpuesta a la señal principal, que será debida, lógicamente, a la vibración natural del sistema. Si se mide la distancia entre dos picos consecutivos de la vibración superpuesta a la fuerza en $x$ se determina que su frecuencia es $5263 \mathrm{~Hz}$, que es precisamente una de las frecuencias naturales de vibración del sistema en el eje $x$.

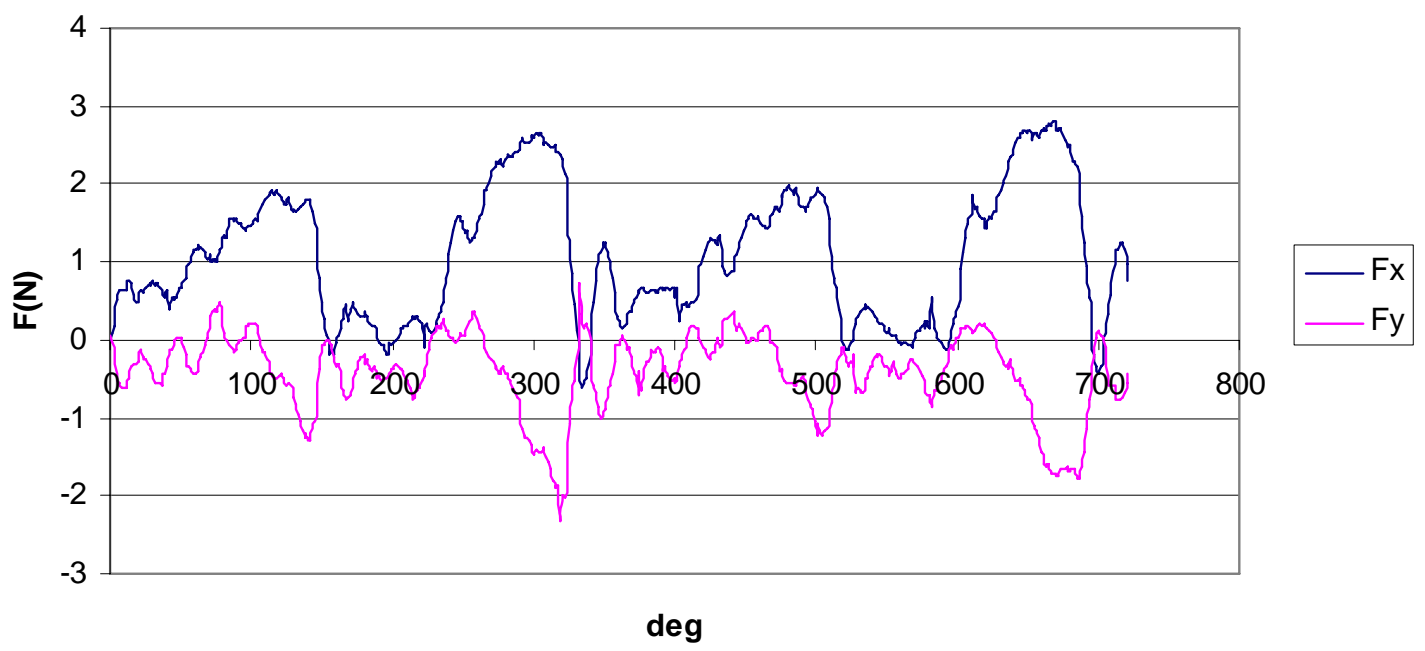

Figura 7.7. Fuerzas del proceso en condiciones de no retemblado. $D=0.4 \mathrm{~mm}, n=$ 23873 r.p.m., $a_{p}=0.15 \mathrm{~mm}, a_{e}=0.2 \mathrm{~mm}, f_{z}=0.003 \mathrm{~mm}$, fresado en oposición

En la figura 7.8 se muestra el espectro de la señal de fuerza en $x$. Se observa claramente el pico principal a la frecuencia de paso de los dientes. Como se indicó más arriba aparece un pico a la frecuencia de giro de la herramienta debido al desequilibrio entre los dientes debido a la desviación excéntrica de la herramienta. Junto a estos picos principales aparecen muchos otros que son armónicos de los primeros, por lo que se dan a múltiplos enteros de la frecuencia principal, es decir, a 1200,1600, $2000 \mathrm{~Hz}$, etc. Las alturas de estos picos son decrecientes con la frecuencia, como es habitual. Sin embargo, se aprecian dos picos que no siguen esta tendencia, situados en 3800 y 5400 $\mathrm{Hz}$ respectivamente. Estos valores corresponden aproximadamente a dos de las frecuencias naturales de vibración del sistema, por lo que se deduce que son debidos a la oscilación libre del sistema, superpuesta a la señal del proceso. 


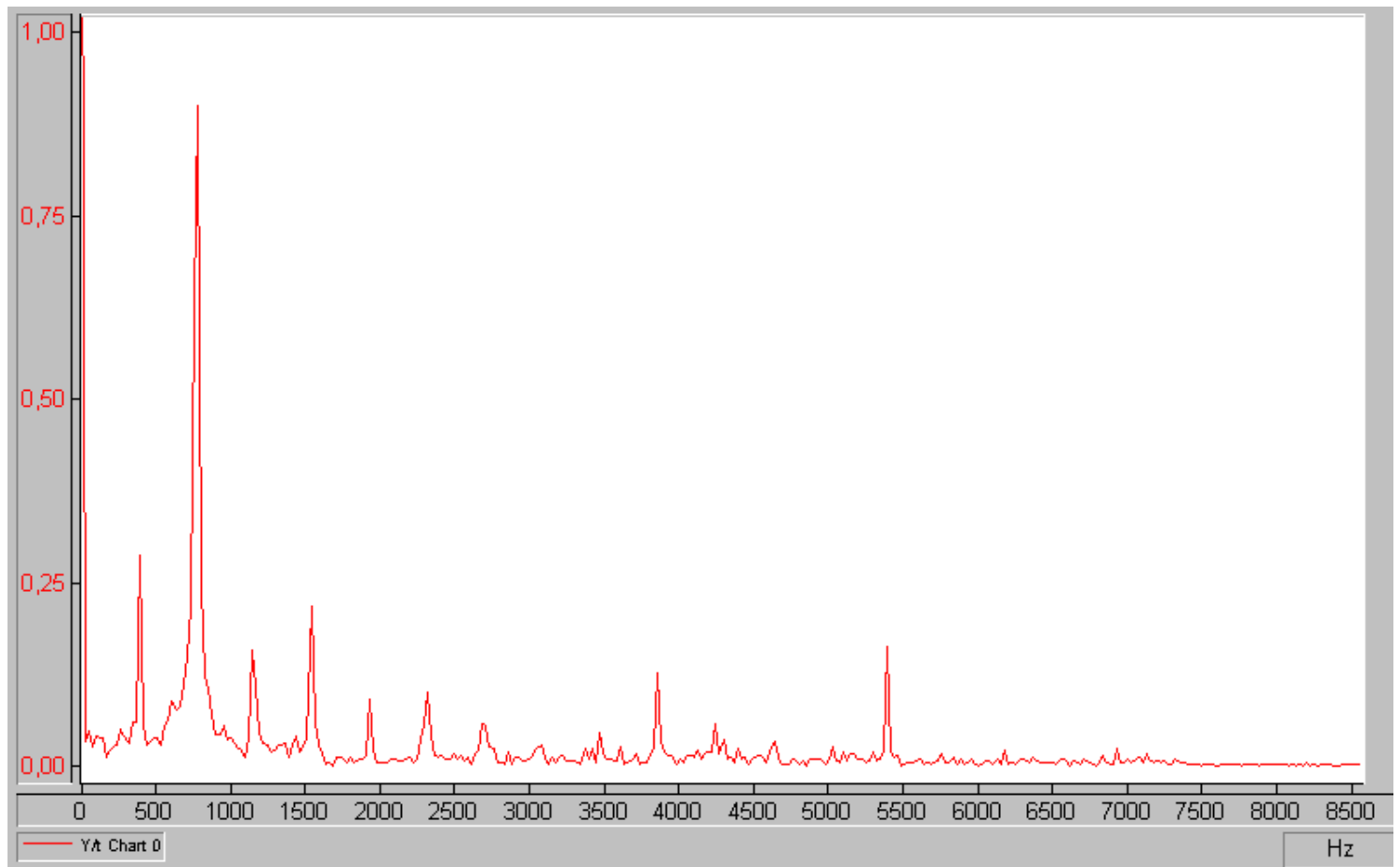

Figura 7.8. Transformada de Fourier de la señal de fuerza en el eje $x . D=0.4 \mathrm{~mm}, n=$ 23873 r.p.m., $a_{p}=0.15 \mathrm{~mm}, a_{e}=0.2 \mathrm{~mm}, f_{z}=0.003 \mathrm{~mm}$, fresado en oposición

En la figura 7.9 se muestran las señales de fuerza obtenidas en el fresado en concordancia, con las mismas condiciones que el ensayo anterior.

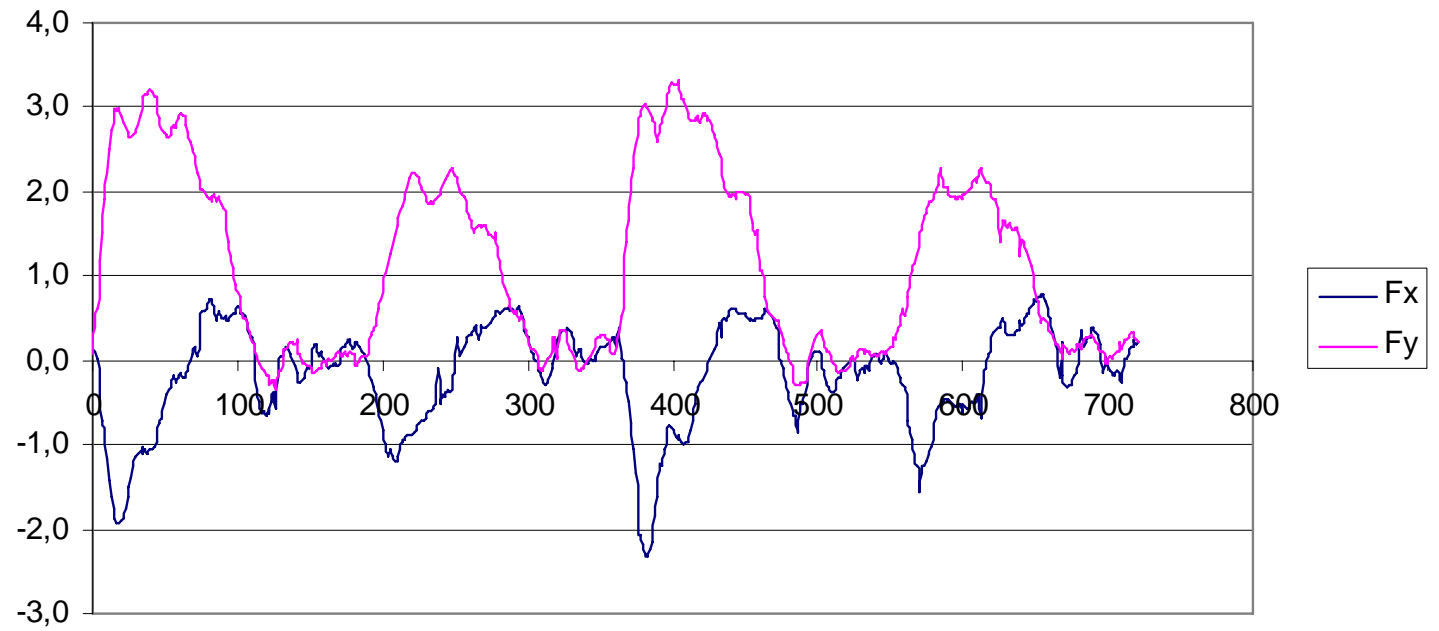

Figura 7.9. Fuerzas del proceso en condiciones de no retemblado. $D=0.4 \mathrm{~mm}, n=$ 23873 r.p.m., $a_{p}=0.15 \mathrm{~mm}, a_{e}=0.2 \mathrm{~mm}, f_{z}=0.003 \mathrm{~mm}$, fresado en concordancia 
En estas señales también se aprecia la influencia de las vibraciones naturales, ya que hay una oscilación superpuesta de la misma frecuencia que la que aparecía en el ensayo mostrado en la figura 7.7.

En la figura 7.10 se muestra el resultado de la transformada de Fourier de la señal de fuerza en el eje $x$ del ensayo en concordancia.

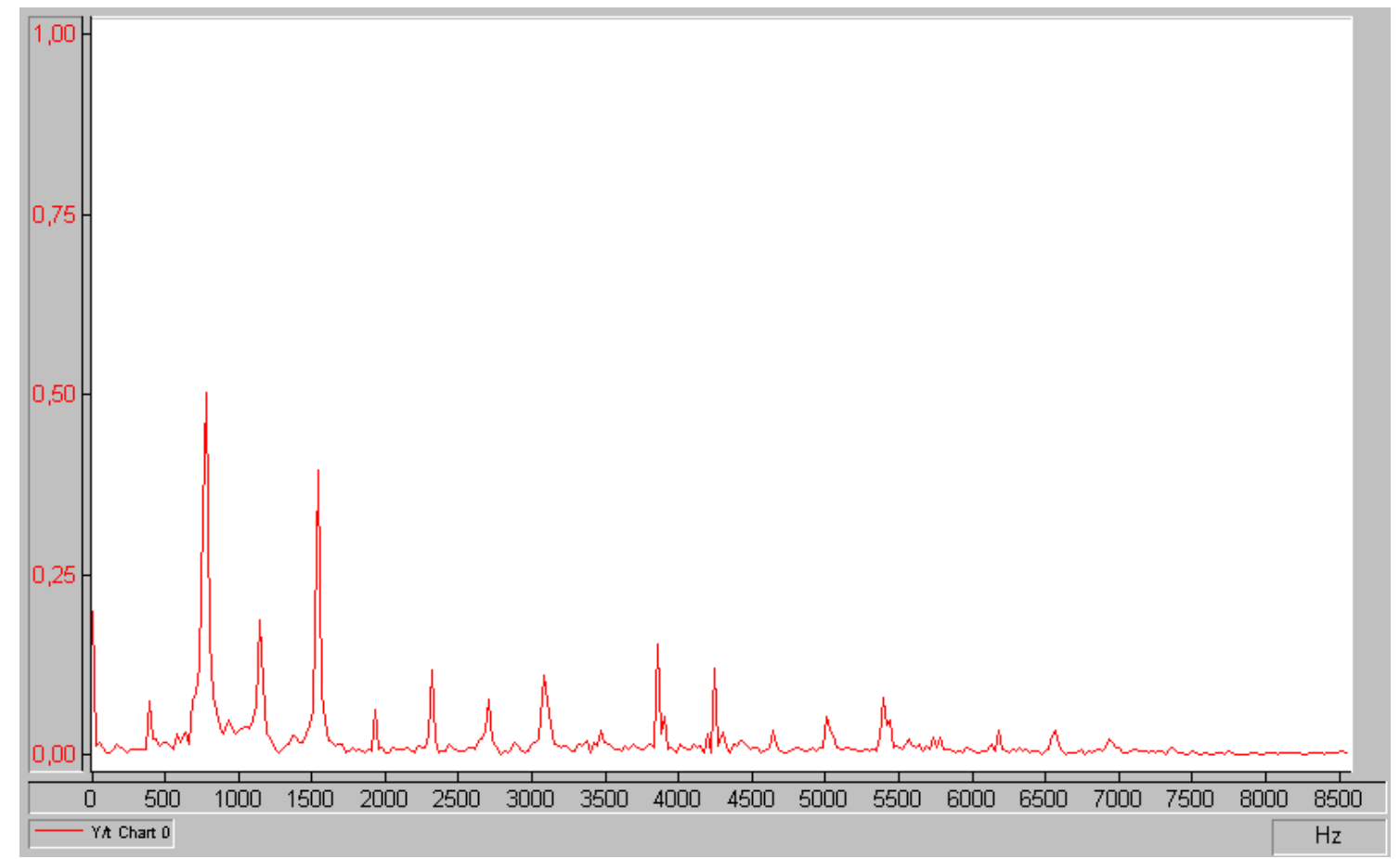

Figura 7.10. Transformada de Fourier de la señal de fuerza en el eje $x . D=0.4 \mathrm{~mm}, n=$ 23873 r.p.m., $a_{p}=0.15 \mathrm{~mm}, a_{e}=0.2 \mathrm{~mm}, f_{z}=0.003 \mathrm{~mm}$, fresado en concordancia

En esta gráfica también se aprecian 2 picos significativos a $3800 \mathrm{~Hz}$ y $5400 \mathrm{~Hz}$, al igual que en el espectro de la señal del fresado en oposición. A diferencia de lo que ocurría en aquélla señal, existen 2 picos principales, en lugar de uno. Esto es debido a que la forma de la primera señal se puede asimilar a una señal senoidal con algunos periodos intermedios de paso por cero, mientras que en la del fresado en concordancia se puede asimilar dos señales senoidales superpuestas, de distintas amplitudes.

\subsection{Filtrado de la señal de fuerzas}


Con el objeto de poder contrastar mejor las señales experimentales de las fuerzas con las simulaciones procedentes del modelo es conveniente eliminar las vibraciones naturales del sistema, ya que no proceden del proceso de fresado, sino del comportamiento dinámico del sistema.

\subsubsection{Filtro paso bajo}

Para ello existen múltiples opciones, entre las cuales está el uso de un filtro paso-bajo aplicado a la señal de fuerzas para atenuar las componentes de la señal que tengan frecuencia superior a un valor determinado. Según se desprende del espectro de la figura 7.8 este valor debería ser $2000 \mathrm{~Hz}$, ya que los armónicos de la señal principal que hay por encima son de muy escaso peso y no se distorsiona demasiado la señal si se suprimen, consiguiendo eliminar a cambio las vibraciones naturales a las frecuencias de 3800 y 5400 Hz, según se ha señalado en el apartado anterior.

En la figura 7.11 se muestra la señal de fuerzas en el fresado en oposición filtrada mediante un filtro paso-bajo a $2000 \mathrm{~Hz}$. Se observa que las señales son más limpias porque se ha eliminado el ruido procedente de las vibraciones naturales. Ello hará que se asemeje más a las señales de predicción obtenidas por el modelo.

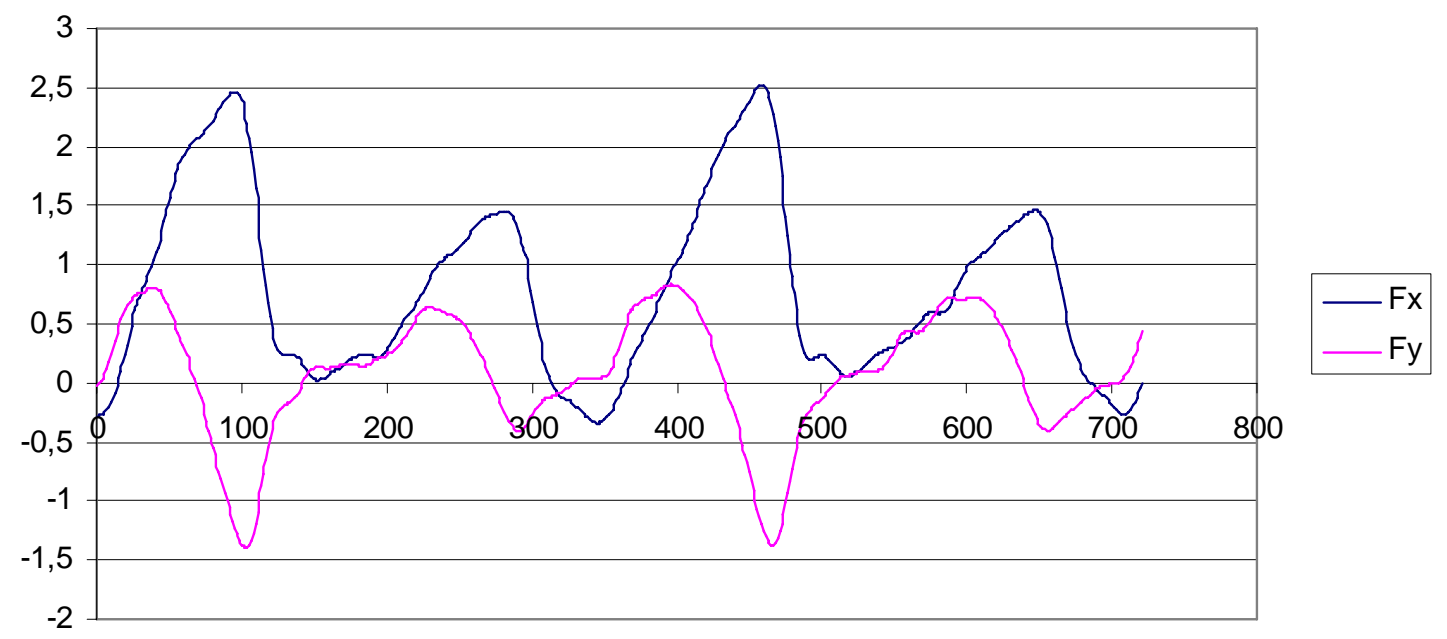

Figura 7.11. Señales de fuerzas filtradas a $2000 \mathrm{~Hz} . \mathrm{D}=0.4 \mathrm{~mm}, n=23873$ r.p.m., $a_{p}=$ $0.15 \mathrm{~mm}, a_{e}=0.2 \mathrm{~mm}, f_{z}=0.003 \mathrm{~mm}$, fresado en oposición 
En la figura 7.12 se muestra el espectro de la señal de fuerza en $x$ filtrada. Se observa que los picos que aparecen por encima de $2000 \mathrm{~Hz}$ han quedado atenuados. Se advierte que el pico de $5400 \mathrm{~Hz}$, correspondiente a las vibraciones naturales del sistema ha pasado a ser de importancia muy pequeña con respecto a la señal principal, debido a que es de frecuencia muy superior a la de corte del filtro y el efecto del filtrado es mayor a medida que crece la frecuencia.

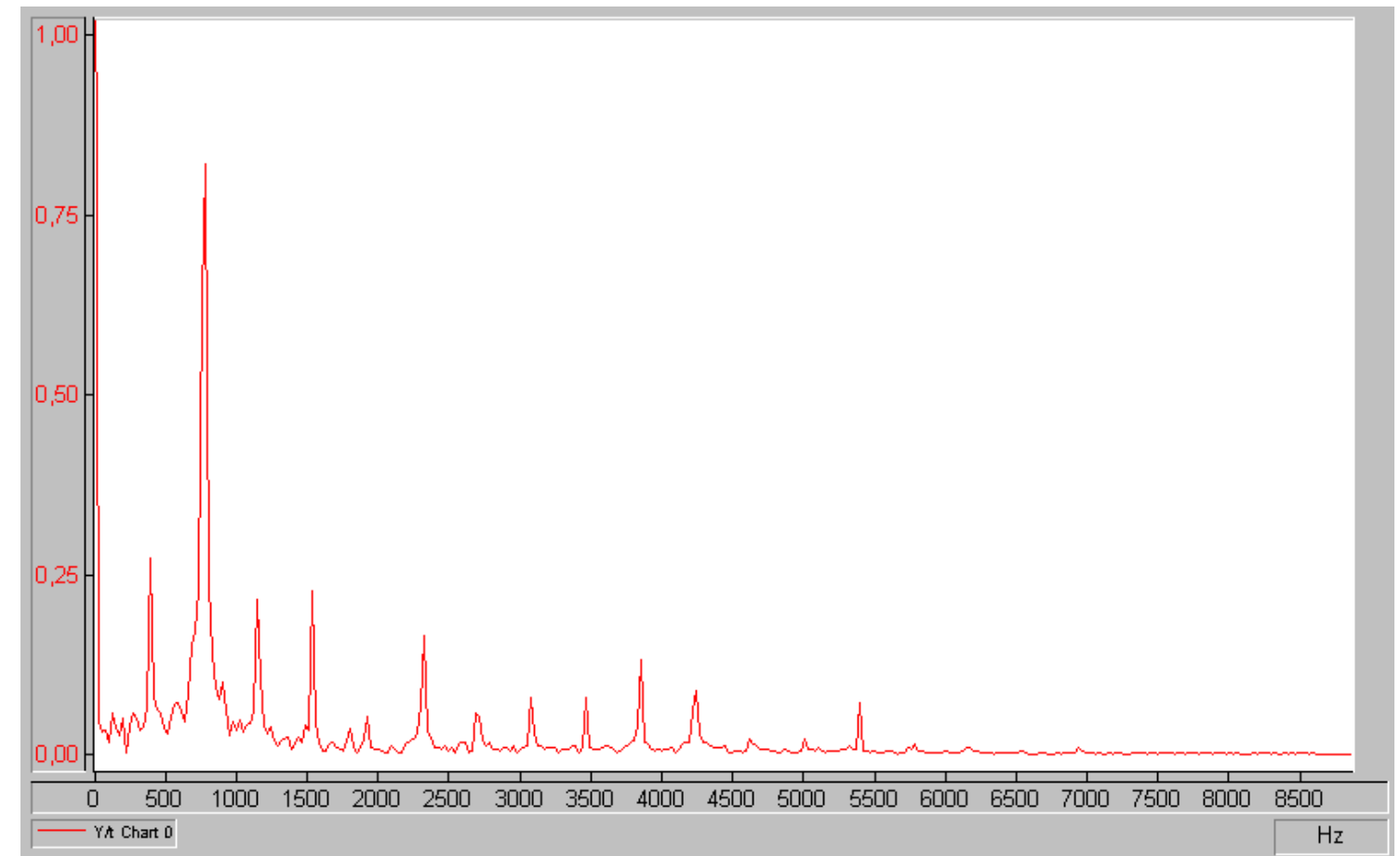

Figura 7.12. Espectro de la señal de fuerza en $x$ filtrada a $2000 \mathrm{~Hz} . D=0.4 \mathrm{~mm}, n=$ 23873 r.p.m., $a_{p}=0.15 \mathrm{~mm}, a_{e}=0.2 \mathrm{~mm}, f_{\mathrm{z}}=0.003 \mathrm{~mm}$, fresado en oposición

En las figuras 7.13 y 7.14 se representa la señal de fuerzas filtrada y el espectro de la fuerza en $x$ para el fresado en concordancia. 


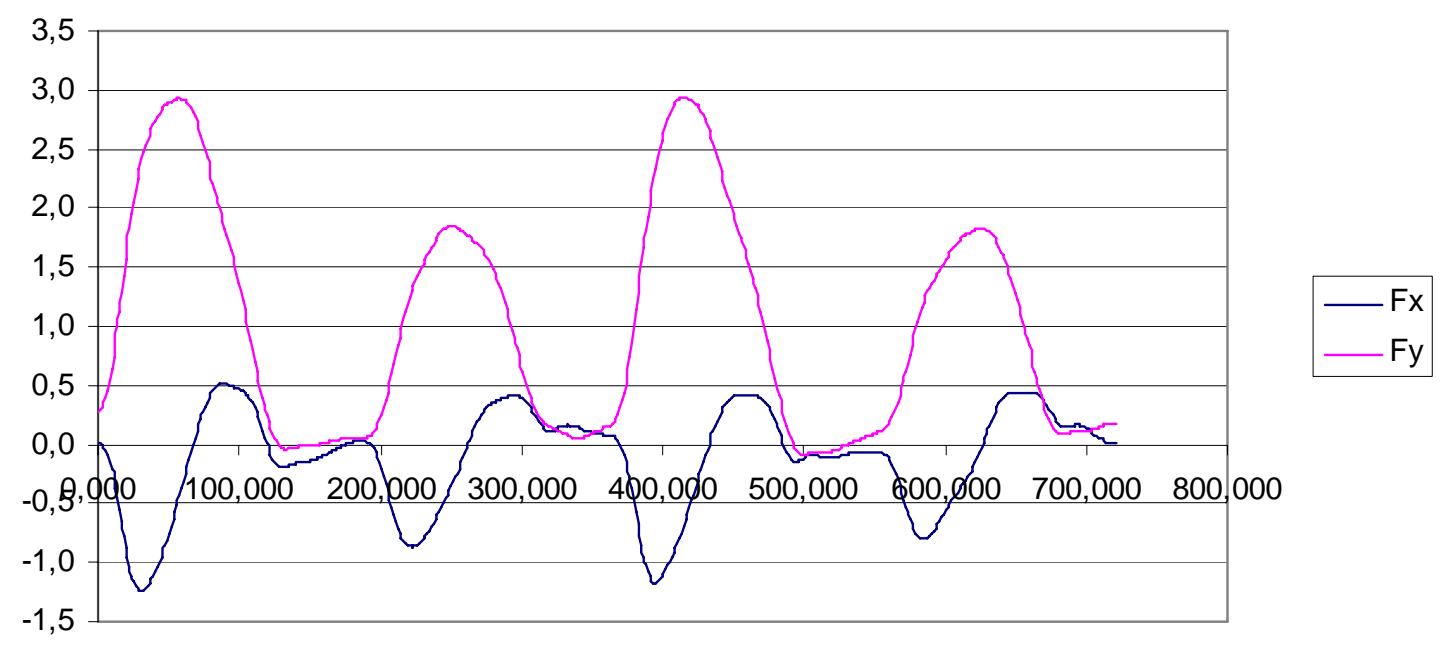

Figura 7.13. Señales de fuerzas filtradas a $2000 \mathrm{~Hz} . \mathrm{D}=0.4 \mathrm{~mm}, n=23873$ r.p.m., $a_{p}=$ $0.15 \mathrm{~mm}, a_{e}=0.2 \mathrm{~mm}, f_{z}=0.003 \mathrm{~mm}$, fresado en concordancia

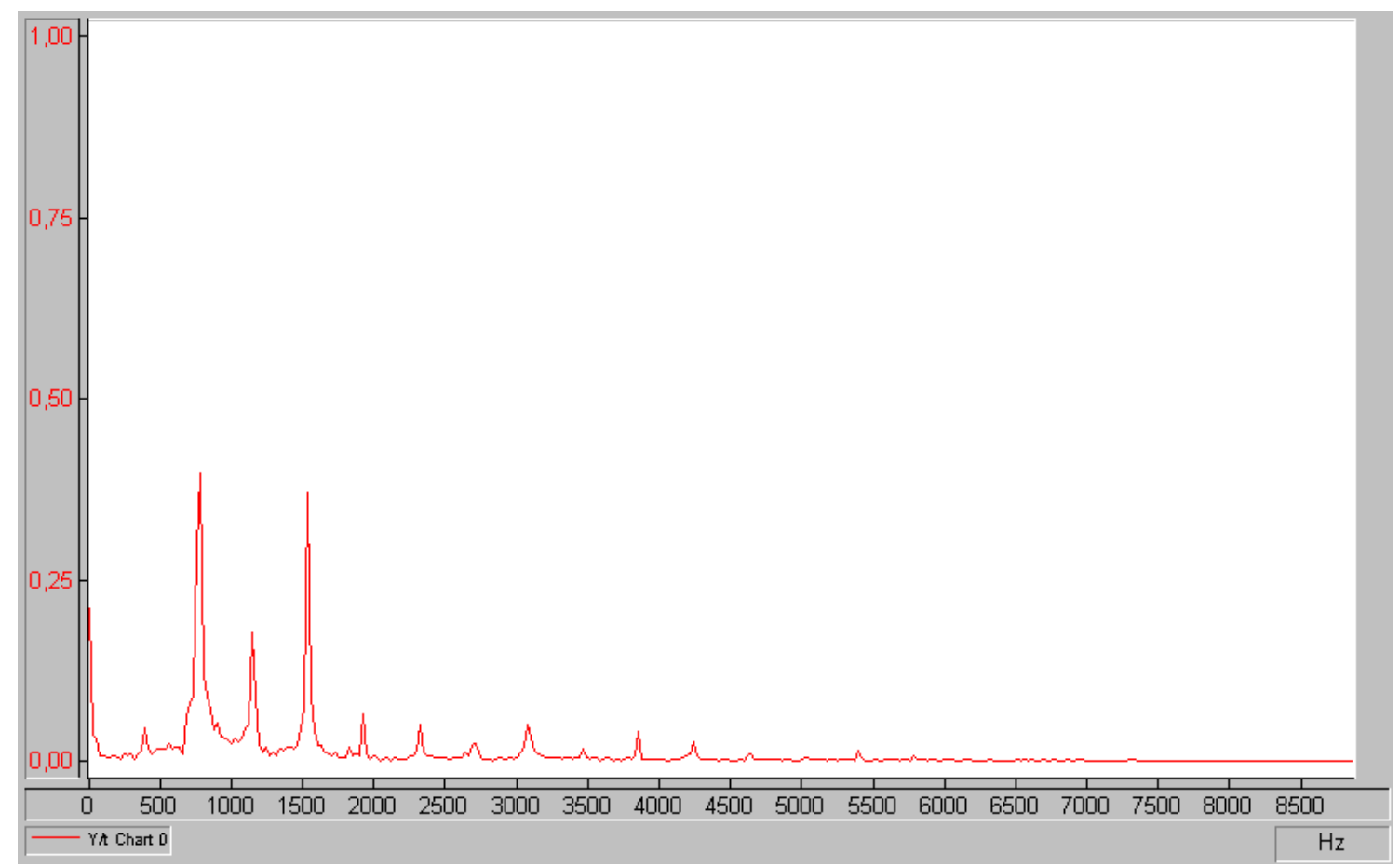

Figura 7.14. Espectro de la señal de fuerza en $x$ filtrada a $2000 \mathrm{~Hz} . D=0.4 \mathrm{~mm}, n=$ 23873 r.p.m., $a_{p}=0.15 \mathrm{~mm}, a_{e}=0.2 \mathrm{~mm}, f_{z}=0.003 \mathrm{~mm}$, fresado en concordancia

En este espectro se observa que los picos de 3800 y $4300 \mathrm{~Hz}$ existentes en la señal original han quedado atenuados en gran medida y por eso la señal es mucho más suave. 


\subsubsection{Métodos alternativos de suavizado}

En general la utilización de un filtro paso-bajo conlleva una pérdida de información, ya que se están eliminando todos los armónicos de la señal que están por encima de la frecuencia de corte. Se ha observado en la figura 7.10 que estos armónicos tienen una amplitud sensiblemente inferior al pico de frecuencia principal, pero si se eliminan todos ellos se causará una distorsión significativa a la señal en su conjunto.

De esa manera se podría pensar en la aplicación de métodos alternativos para el suavizado de la señal: un filtro Kalman o un filtro inverso, dado que son sistemas habituales para la eliminación de ruido en señales de diversos tipos.

El filtro Kalman podría ser una opción adecuada para eliminar el ruido sin distorsionar la señal, ya que este proceso realiza un estudio estadístico del valor de la señal a lo largo del tiempo, proporcionando una comparación entre el valor medido y el valor esperado [109]. De esta manera, podría descontarse el ruido del valor medido sin perjudicar la señal real.

Sin embargo, su uso está limitado a las funciones definidas recursivamente, mediante expresiones como la siguiente:

$$
X_{t}=A \cdot X_{t-1}+w_{t-1}
$$

A esta función suele ir asociada la correspondiente a las medidas de la señal:

$$
Z_{t}=H \cdot X_{1}+v_{t}
$$

Esta forma de definir la función no se puede adaptar a la predicción de las fuerzas ni a su medida en el proceso de microfresado, ya que cada valor no depende del valor anterior sino de la posición de la herramienta y su superficie de contacto con la pieza.

Otro método en el que se podría pensar es la técnica del filtrado inverso, ya que es un buen modo de eliminar rasgos no deseados de una señal producidos por una 
determinada distorsión mediante la aplicación de la función de transferencia inversa de dicha distorsión a la señal [110]. El proceso se puede representar mediante el esquema de la figura 7.15 .

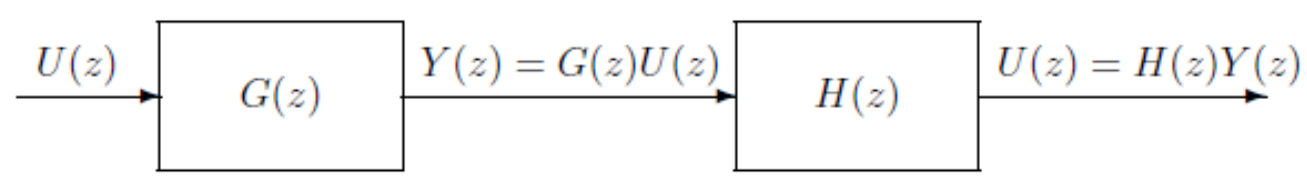

Figura 7.15. Esquema de aplicación del filtrado inverso

Si las vibraciones naturales del sistema se asimilasen a un bloque adicional que introduce variación en la señal y cuya función de transferencia fuera $H(z)$, se podría introducir a la salida del diagrama representado en la figura 7.15 un bloque cuya función de transferencia fuera el valor inverso, que vendría dado por la expresión:

$$
G(z)=\frac{1}{H(z)}(7.4)
$$

En el caso de la señal de fuerzas del microfresado la distorsión procede del comportamiento dinámico del sistema herramienta-pieza, cuya función de transferencia se desconoce por falta de equipamiento adecuado, aunque la medición de los parámetros dinámicos del sistema es el objeto de una futura línea de investigación que se llevará a cabo para desarrollar un modelo dinámico de fuerzas en el microfresado.

Se ha de recurrir, por tanto, a otra técnica de filtrado para adaptar las medidas experimentales del proceso al modelo estático desarrollado en el presente trabajo.

Otra posible solución de filtrado de la señal es la implementación de un filtro de banda eliminada o "notch-filter", que elimina sólo un estrecho rango de frecuencias en el espectro dejando sin cambios el resto. De esta manera, se podría retirar de la señal sólo la componente de vibraciones naturales del sistema obteniéndose como resultado la señal pura correspondiente al proceso de corte. 
El modo de usar esta técnica es hacer que la señal pase por dos filtros en paralelo: un filtro paso-alto cuya frecuencia de corte sea ligeramente superior a la que se pretende eliminar y un filtro paso-bajo con frecuencia de corte ligeramente inferior a la que se intenta suprimir. El diagrama correspondiente a esta técnica se muestra en la figura 7.16.

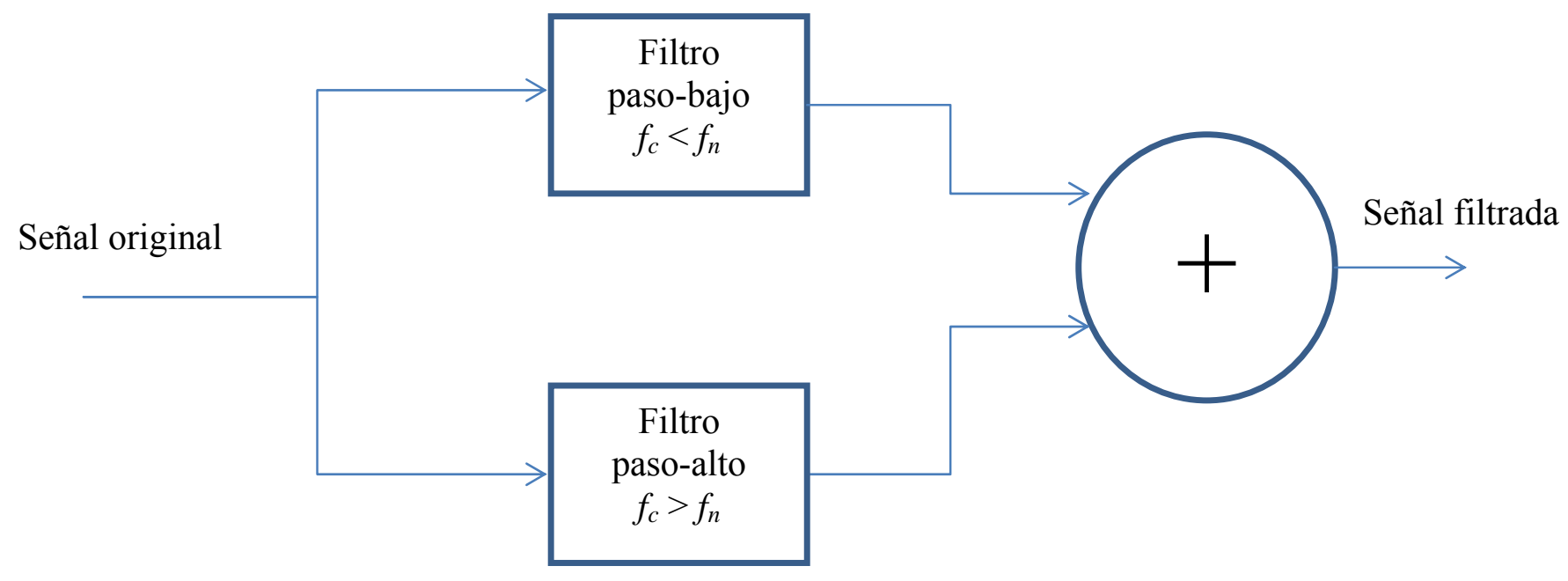

Figura 7.16. Esquema del filtro de banda eliminada

Este método es útil para reducir las vibraciones pero no consigue eliminarlas totalmente, dado que la frecuencia de corte de los filtros se debe mantener muy cercana a la frecuencia natural de vibración del sistema para no distorsionar el resto de la señal. Como los filtros atenúan escasamente los picos de frecuencia cercana a la frecuencia de corte, la reducción de las vibraciones con esta técnica no es muy drástica.

Otra manera de eliminar las vibraciones naturales de la señal sería suprimir el pico correspondiente a ellas en el espectro de la Transformada de Fourier de la señal y posteriormente realizar la Transformada Inversa. Este método es conceptualmente sencillo pero difícil de llevar a la práctica porque se necesita cambiar el valor de la Transformada de Fourier correspondiente a la frecuencia natural por otro valor y no es fácil deducir este valor de tal manera que no se distorsione la señal original.

Una forma alternativa de reducir las vibraciones naturales significativamente es el método del valor promedio. Consiste en tomar varios ciclos de la señal y obtener el valor medio. Estos ciclos incluyen a su vez las fuerzas correspondientes a varias vueltas 
de la herramienta. Al obtener el valor medio se elimina el "ruido" provocado por las vibraciones, ya que su frecuencia no es un múltiplo entero de la frecuencia principal de la señal y por tanto presentarán un valor aleatorio en cada ciclo, consiguiéndose así la eliminación de las señales que no tengan la misma frecuencia que la señal principal.

Existe una dificultad de orden práctico con este método y proviene del hecho de que la señal se mide mediante un sistema de adquisición digital con una frecuencia de muestreo determinada. Si la frecuencia principal de la señal fuera un múltiplo entero de la frecuencia de muestreo los valores muestrales en cada ciclo estarían en la misma posición relativa respecto al inicio del ciclo, ya que todos los valores de los distintos ciclos estarían desplazados un periodo, que sería múltiplo entero de la frecuencia de muestreo, de tal manera que se podrían "superponer" los valores de un ciclo con los de otro sin variar su posición temporal. Dado que en general no se da este caso, los valores muestrales de los diferentes ciclos no corresponderán a la misma posición relativa. Para resolver este problema se ha de realizar una interpolación de los valores muestrales a posiciones temporales determinadas dentro del periodo. De esa manera se podrá obtener el valor medio de las muestras de distintos ciclos correspondientes a la misma posición temporal relativa al inicio del ciclo.

Éste es el método que se ha utilizado en este trabajo para la eliminación de las vibraciones naturales del sistema por ser sencillo de aplicar y proporcionar buenos resultados.

En las gráficas de las figuras 7.17 y 7.18 se puede apreciar el efecto que ejerce el método del valor promedio sobre una señal de fuerzas tomada del proceso con vibraciones. 


\section{Señal original}

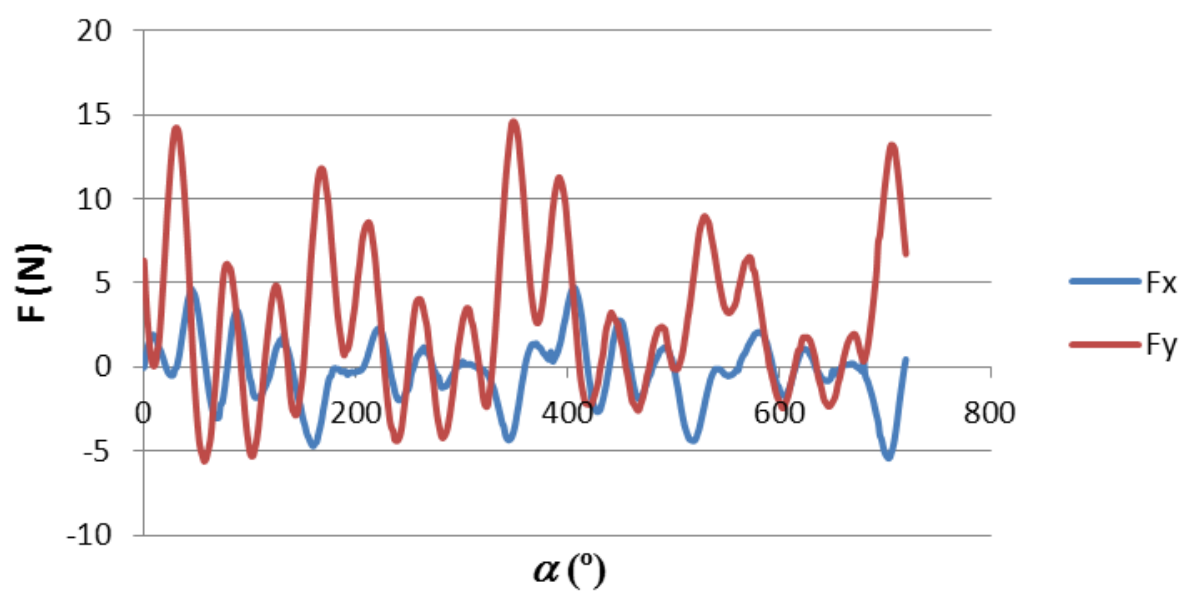

Figura 8.17. Señal original del proceso

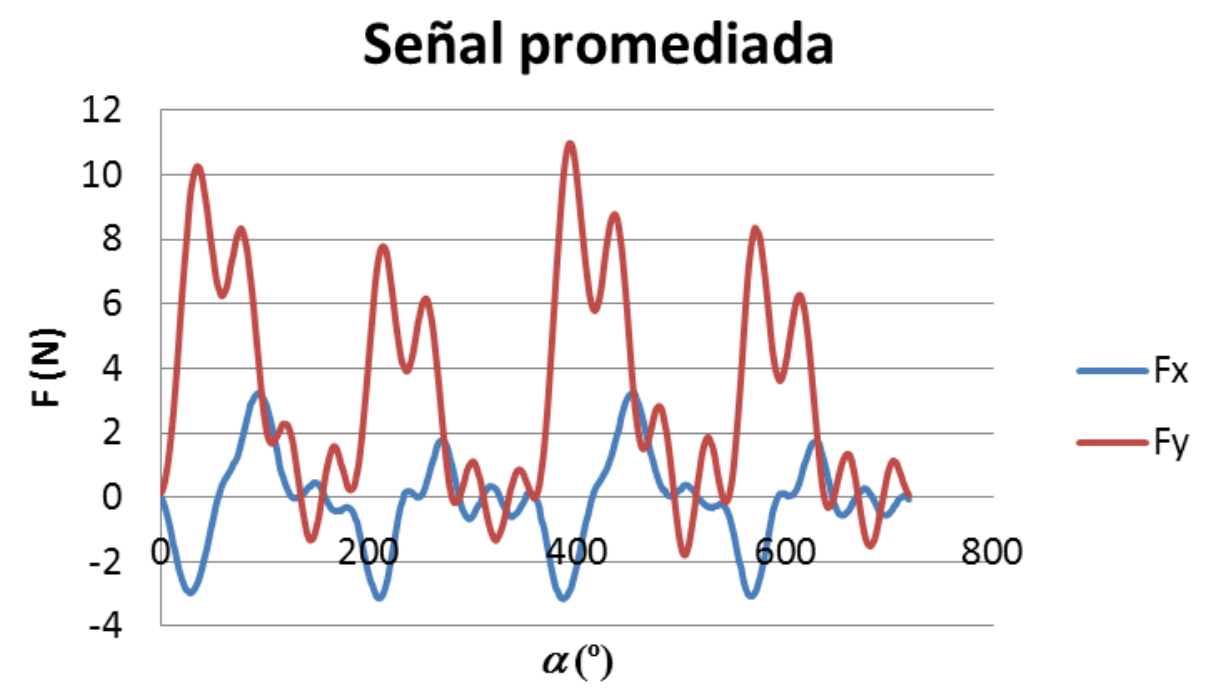

Figura 7.18. Señal de fuerzas promediada

A continuación se mostrarán otros resultados experimentales a los que se ha aplicado este método. El buen grado de ajuste que se observa entre el modelo predictivo de fuerzas y las medidas promediadas es otra razón que avala la utilización de este método de filtrado.

Para evaluar el efecto de las vibraciones puede ser útil también la comparación de la señal de fuerza original con la simulación procedente del modelo, ya que en ella no habrá vibraciones naturales, mientras que en la señal promediada sí existen, aunque 
atenuadas. Esta comparación con el modelo se realizará con las señales originales y las filtradas para apreciar la diferencia. En las figuras 7.19 y 7.20 se muestran las señales de fuerzas para el ensayo de fresado en oposición descrito en el capítulo 3 junto con las predicciones del modelo.

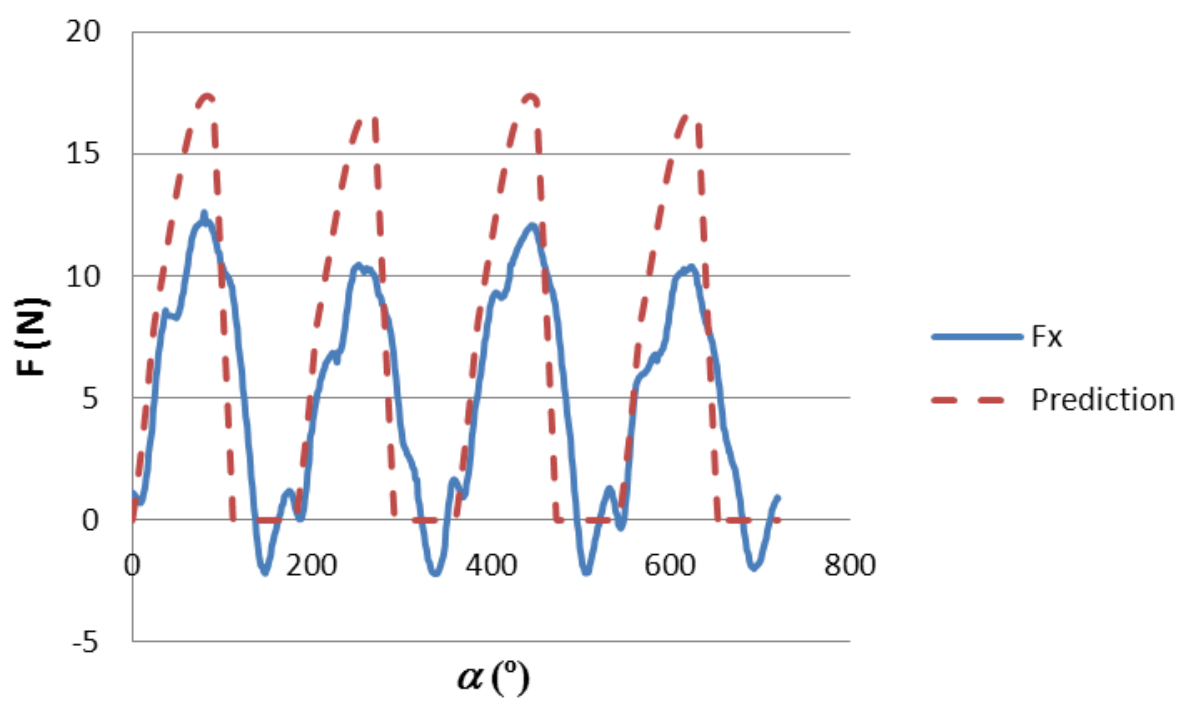

Figura 7.19. Comparación de la señal de fuerza en $x$ con la simulación para el fresado en oposición

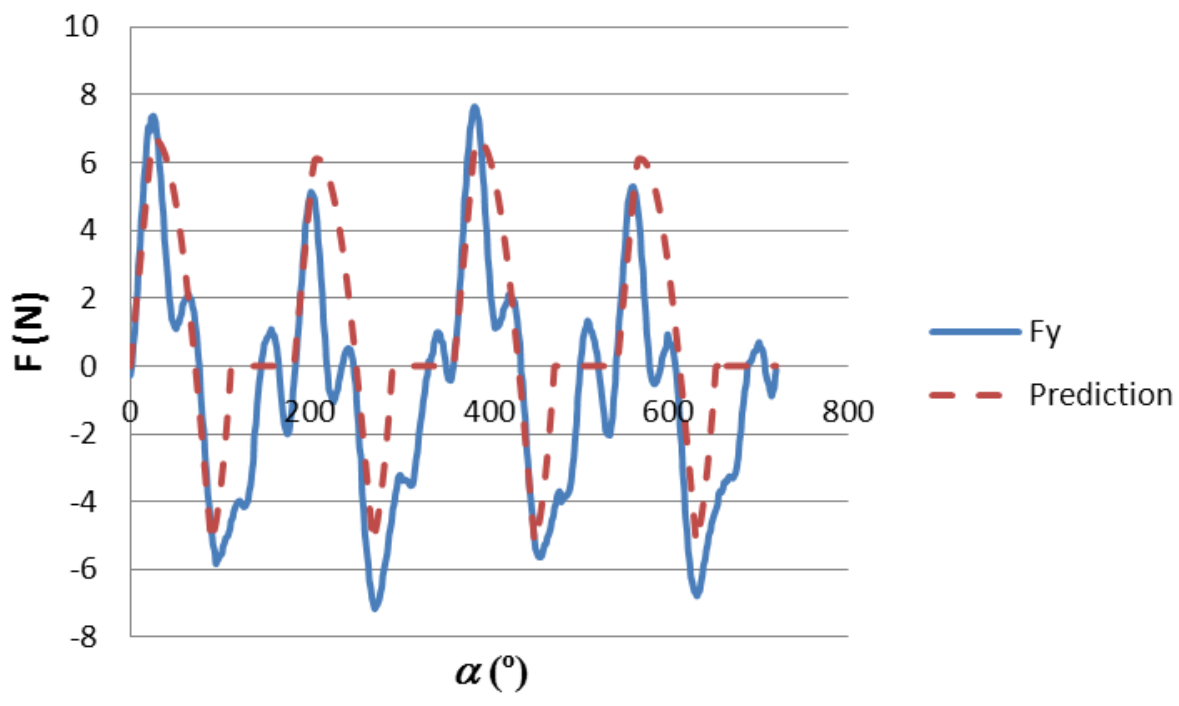

Figura 7.20. Comparación de la señal de fuerza en $y$ con la simulación para el fresado en oposición 
Se puede observar que las vibraciones distorsionan en gran medida la señal y provocan falta de ajuste con la predicción. Las vibraciones se aprecian especialmente en los periodos de paso por 0 , en los que la señal experimental corresponde a la oscilación natural de la pieza, mientras que en la señal del modelo el valor será nulo. En esos periodos se observan picos de magnitud similar a la de la señal original, por lo que el ajuste con el modelo es particularmente deficiente.

Aplicando el filtrado visto en el apartado anterior la similitud entre ambas señales es mucho mayor. En las figuras 7.21 y 7.22 se muestran las gráficas de las señales de fuerzas experimentales y simuladas del ensayo descrito en el apartado 3.

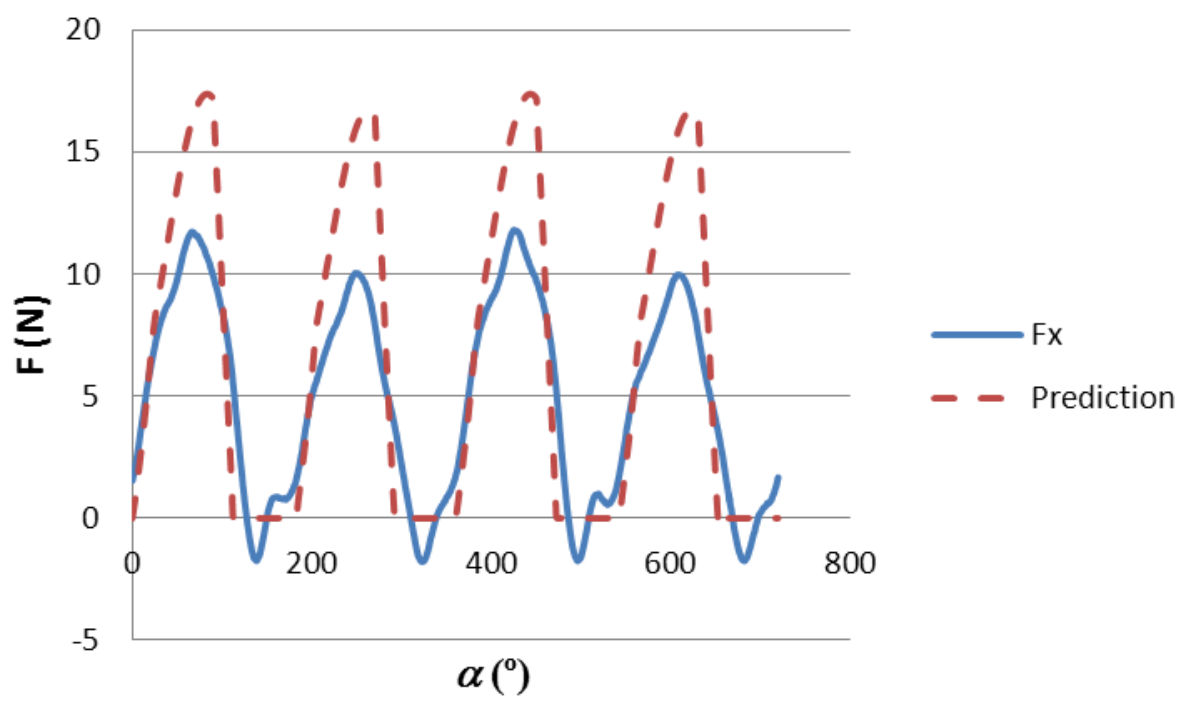

Figura 7.21. Comparación de la señal de fuerza en $x$ filtrada con la simulación para el fresado en oposición 


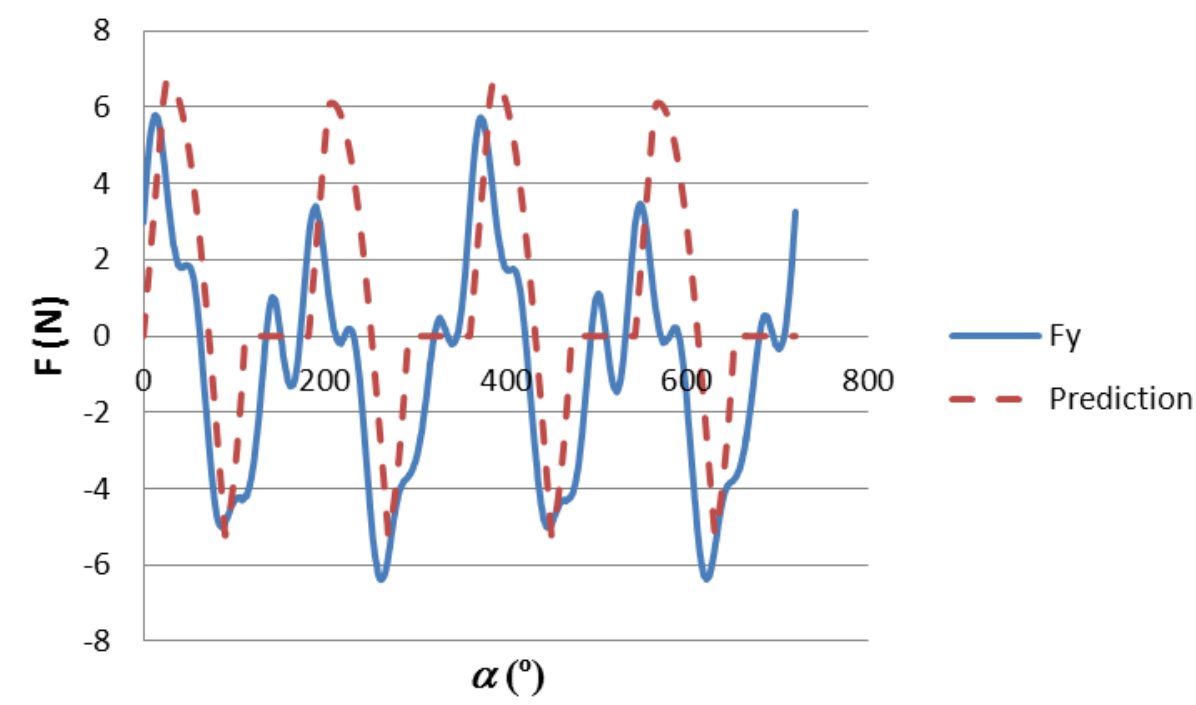

Figura 7.22. Comparación de la señal de fuerza en y filtrada con la simulación para el fresado en oposición

En estas gráficas se aprecia cualitativamente un mejor ajuste entre la predicción y la señal experimental, aunque sigue habiendo diferencias sensibles debido a que las vibraciones no se han eliminado totalmente.

En las figuras 7.23-7.26 se realiza la comparación entre señales experimentales y simuladas para fresado en concordancia. En este caso también se aprecia un mejor ajuste cualitativo con las señales filtradas. 


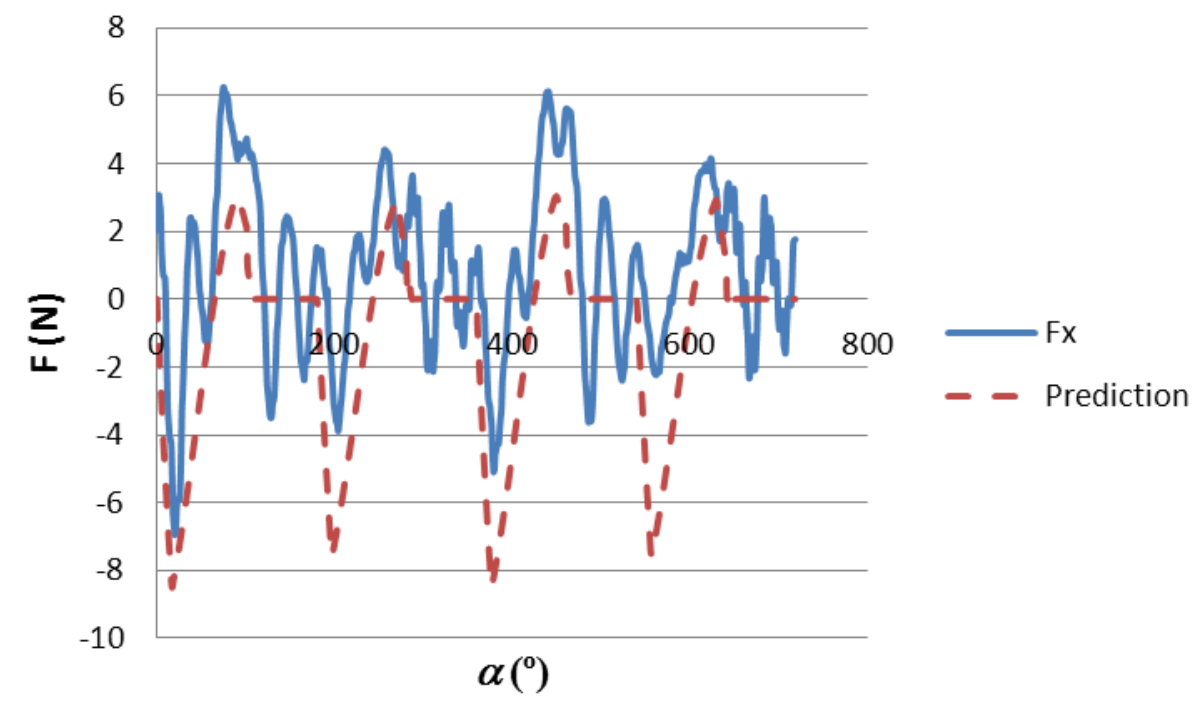

Figura 7.23. Comparación de la señal de fuerza en $x$ con la simulación para el fresado en concordancia

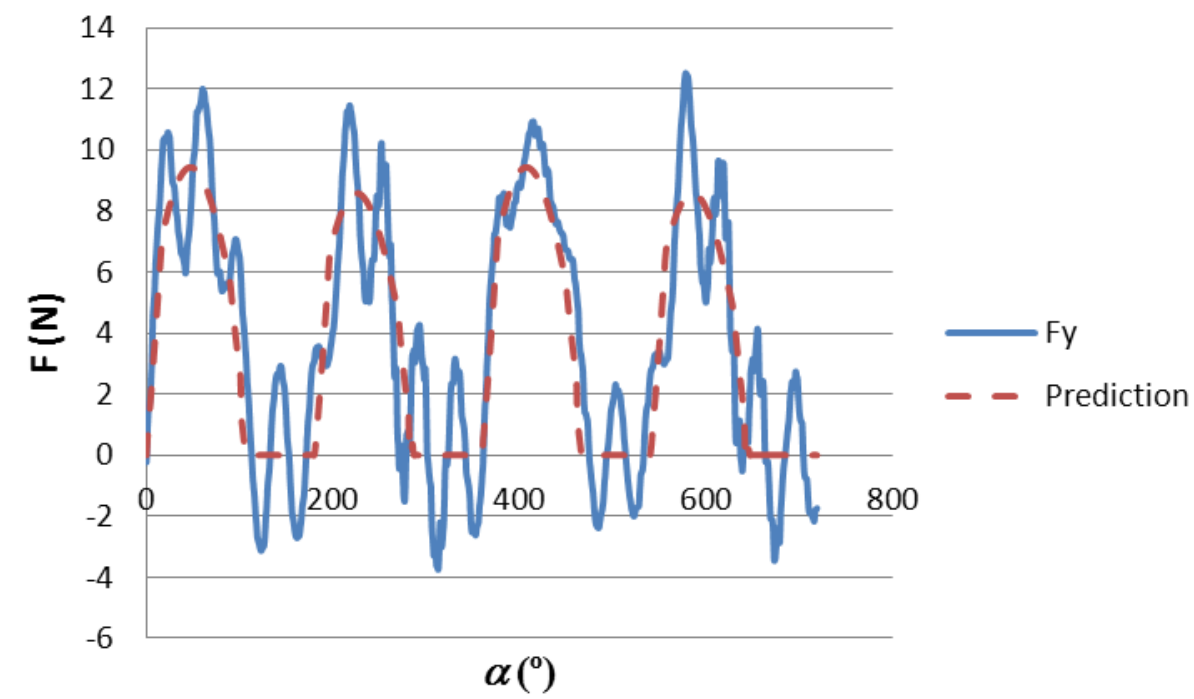

Figura 7.24. Comparación de la señal de fuerza en $y$ con la simulación para el fresado en concordancia 


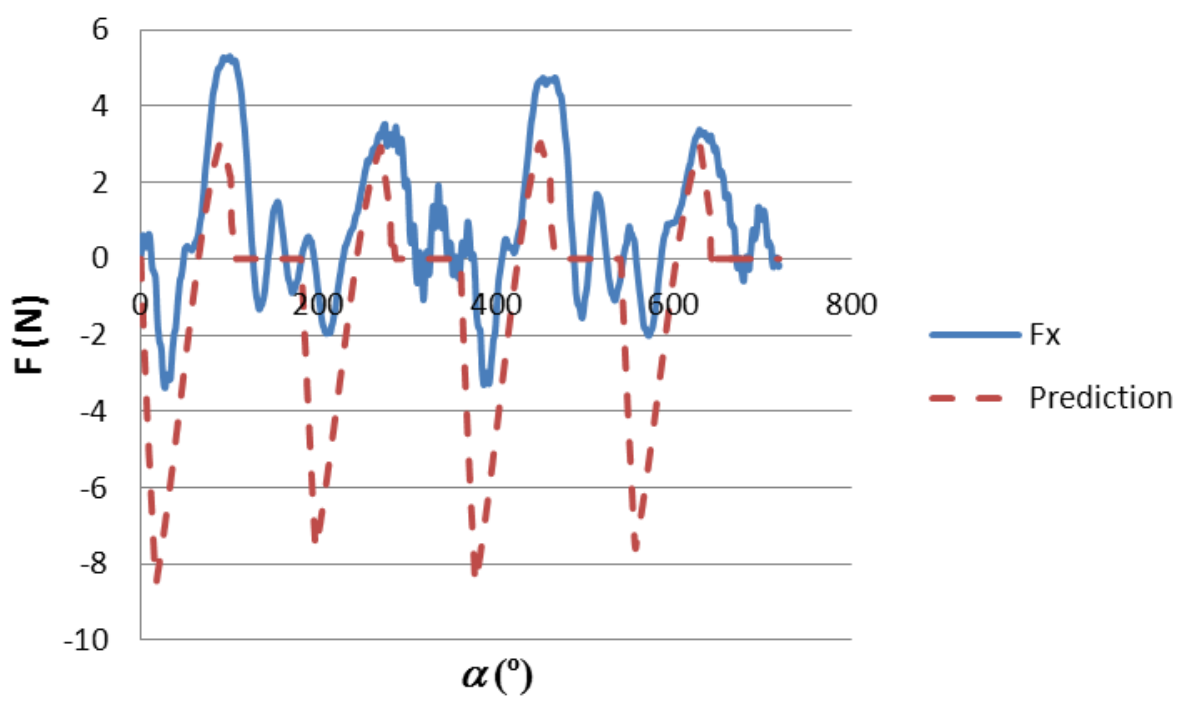

Figura 7.25. Comparación de la señal de fuerza en $x$ promediada con la simulación para el fresado en concordancia

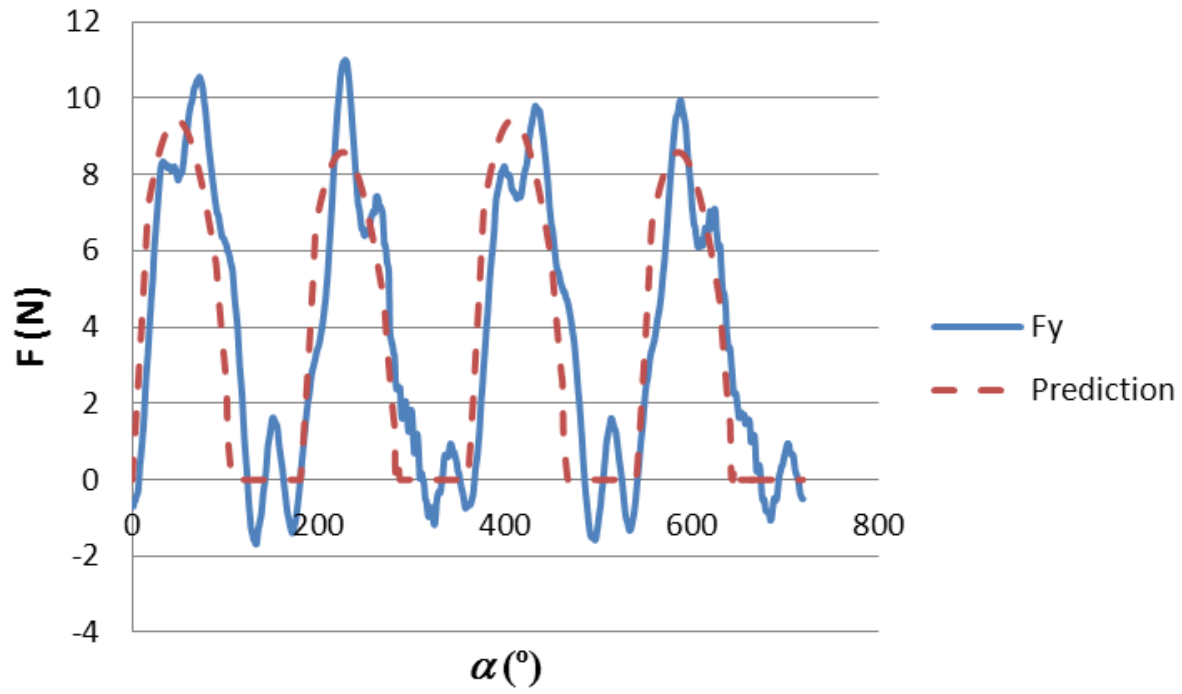

Figura 7.26. Comparación de la señal de fuerza en y promediada con la simulación para el fresado en concordancia 


\section{APLICACIONES DEL MODELO}

\subsection{Monitorización del proceso}

El proceso de microfresado es muy sensible a los cambios en condiciones de corte, ya que el desgaste de la herramienta depende mucho de estas condiciones, especialmente la velocidad de corte, y a su vez un aumento rápido del desgaste provoca un aumento drástico en las fuerzas de corte e incluso la rotura de la herramienta en pocos segundos [94].

A partir de una buena correlación entre el desgaste de herramienta y las fuerzas de corte, la monitorización de dichas fuerzas puede ser un método muy adecuado para la estimación del desgaste de herramienta y consecuentemente un medio para evitar la rotura súbita, que provocaría un serio inconveniente en los tiempos y rendimiento de la fabricación.

En esta técnica el modelo analítico de fuerzas tendría gran relevancia, ya que permitiría realizar una correcta correlación entre el valor de las fuerzas y el desgaste, utilizando como índices los coeficientes de las fuerzas, generados en la fase de determinación de parámetros, según se explica en el capítulo 6 .

\subsection{Control Adaptativo}

\subsubsection{Descripción del sistema}

El Control Adaptativo es una aplicación especialmente idónea del Modelo Predictivo de Fuerzas, ya que éste es una pieza clave del bucle de realimentación, que permite obtener los parámetros óptimos de control de manera sencilla y fiable. Este tipo de control es muy útil en procesos de microfresado cuando las condiciones son cambiantes, es decir, cuando varían los parámetros de la profundidad axial o radial a lo largo del proceso, como suele ser habitual en piezas de geometría complicada. 
En el microfresado existen diversas restricciones físicas al proceso. Algunas de estas restricciones son debidas a la máquina herramienta, como el par máximo o la potencia máxima que proporciona el husillo. Otras restricciones son debidas a la herramienta o a la pieza. Como se ha señalado en el apartado anterior, se debe evitar un valor excesivo de espesor de viruta para que la fuerza que ejerce la herramienta no supere la resistencia a la rotura. En este apartado se describirá la manera de llevar a cabo el control adaptativo de un proceso de microfresado para mantener la fuerza de corte por debajo de un valor de seguridad, actuando sobre el avance por diente para influir sobre el espesor de viruta.

En la figura 8.1 se muestra un diagrama de bloques sencillo que esquematiza el control del proceso. La entrada al sistema es la referencia o valor máximo deseado para la fuerza. Las fuerzas reales que tienen lugar en el proceso se miden mediante un dinamómetro con suficiente frecuencia de muestreo, del tipo descrito en el capítulo 1, con el cual se registran datos de las fuerzas cada 3 ó $5^{\circ}$ de giro del husillo, con los que se evalúa el pico de la fuerza de corte en cada periodo de paso de diente. Cuando la fresa tiene muchos dientes es aconsejable utilizar el valor máximo de las fuerzas por cada vuelta del husillo, de lo contrario la desviación excéntrica de la herramienta hará que los picos de fuerza oscilen de un diente a otro y que el valor resultante del avance por diente que genera el control sea oscilatorio, lo cual no es deseable a efectos de durabilidad de la máquina. Por tanto, obtenido el pico de la fuerza para cada vuelta de husillo $\left(F_{P}(k)\right)$, se resta del valor de referencia de la fuerza $\left(F_{r}(k)\right)$. 


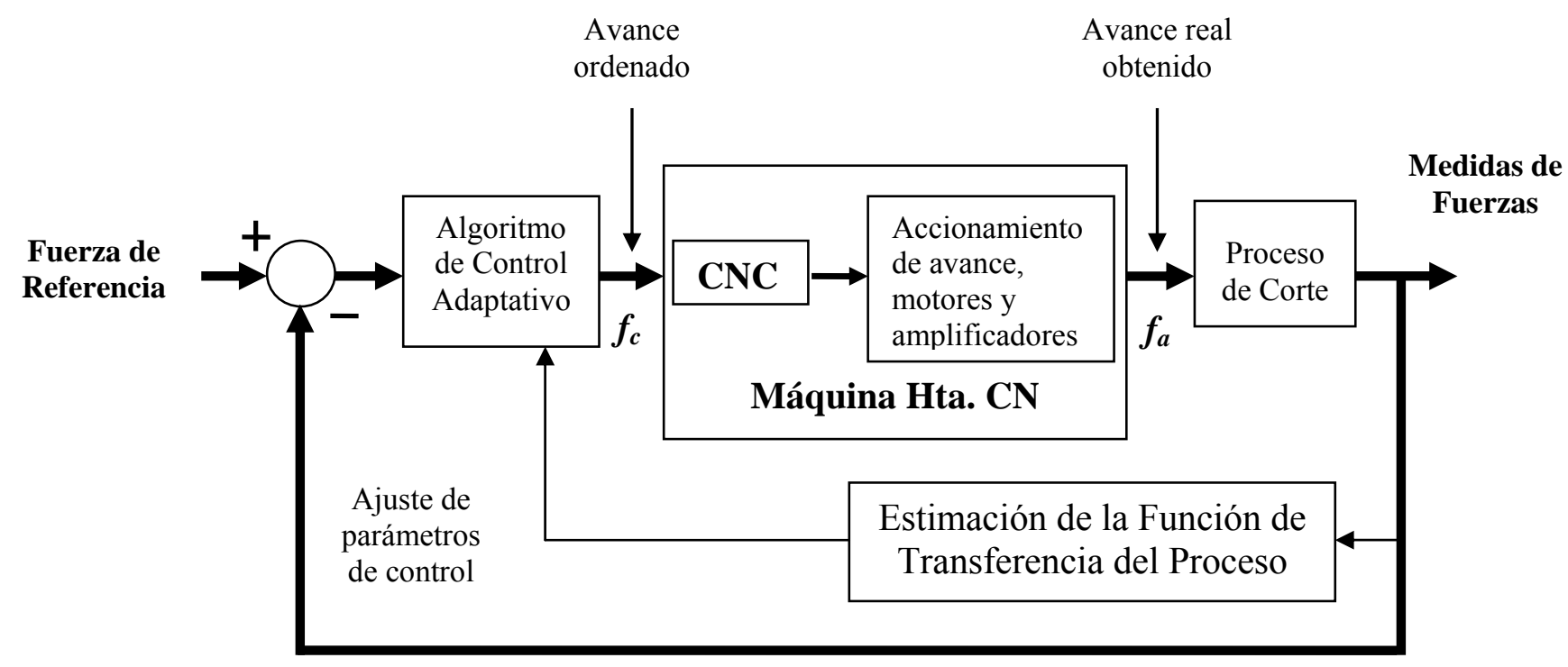

Figura 8.1. Esquema del control adaptativo empleado para limitar la fuerza de corte

El algoritmo de control adaptativo determina un nuevo valor del avance para minimizar el error en la fuerza. El avance obtenido $\left(f_{c}(k)\right)$ se envía a la unidad de control numérico, que tiene su propio bucle de control digital de posición, ejecutado a intervalos de tiempo relativamente pequeños $(0.1 \mathrm{~ms})$. El control numérico envía la señal de tensión correspondiente a los accionamientos de los motores de la máquina, que desplazarán la mesa a una velocidad real $f_{a}$. Los servomecanismos de la máquina están ajustados normalmente para ser sobreamortiguados, por lo que pueden ser considerados como un sistema dinámico de primer orden. Un valor típico de la constante de tiempo de estos sistemas es $0.1 \mathrm{~ms}$, pero podría ser mucho menor en máquinas de alta velocidad. El proceso de corte detecta el cambio en el espesor de viruta después de por lo menos el periodo correspondiente a un paso de diente, y por tanto, el valor máximo de la fuerza experimentará el cambio tras ese periodo. Si el proceso de corte es sin retemblado puede ser considerado también como un sistema de primer orden con una constante de tiempo al menos de un paso de diente, aunque siempre menor que una vuelta de husillo. Cuando hay cambios en la profundidad axial o radial de corte la fuerza máxima de corte variará a lo largo del recorrido de la herramienta, lo cual significa que los parámetros que caracterizan el sistema del proceso de corte varían. El conjunto control numérico, accionamientos de la máquina y proceso de corte puede aproximarse mediante un sistema de segundo orden con función de transferencia $G_{c}(s)$. La fuerza máxima se 
evalúa a partir de las mediciones de fuerzas del proceso y este valor es enviado a un algoritmo de identificación de proceso, en el que se estiman los parámetros digitales que caracterizan el sistema a medida que la herramienta recorre los orificios, ranuras y otras zonas de la pieza. Estos coeficientes se obtienen mediante comparación entre el avance por diente introducido y el valor máximo de fuerza medido. Los coeficientes se utilizarán para actualizar los parámetros del control adaptativo en cada intervalo. Dado que los parámetros del control se ajustan automáticamente según los cambios en la geometría de corte se dice que el sistema de control es adaptativo. El control proporciona un valor del avance que influye en el espesor de viruta cortado y éste a su vez en la fuerza máxima de corte ejercida por la herramienta. El bucle de control adaptativo asegura que esta fuerza sea razonablemente cercana al valor de referencia prefijado, y a su vez, este valor se establecerá con un cierto margen de seguridad por debajo de la fuerza de rotura de la herramienta. A continuación se describirán los pasos del diseño de un controlador mediante la localización de polos.

\subsubsection{Función de Transferencia Discreta del sistema de control}

Como se indicó en el apartado anterior los accionamientos de la máquina pueden aproximarse por un sistema continuo de primer orden:

$$
G_{m}(s)=\frac{f_{a}(s)}{f_{c}(s)}=\frac{1}{\tau_{m} s+1}
$$

Donde $f_{a}$ y $f_{c}$ son respectivamente la entrada y la salida en el sistema de la velocidad de avance en $\mathrm{mm} / \mathrm{s}$. El avance por diente vendrá dado por la siguiente expresión:

$$
h=\frac{f_{c}}{Z \cdot n}
$$

Donde $Z$ es el número de dientes de la fresa y $n$ la velocidad de giro del husillo en rev/s. Dado que la fuerza no varía instantáneamente cuando tiene lugar el cambio de avance, se trata de un sistema, como se ha indicado anteriormente. Este sistema puede ser aproximado mediante una función de transferencia de primer orden: 


$$
G_{p}(s)=\frac{F_{p}(s)}{f_{a}(s)}=\frac{K_{c} \cdot a \cdot b\left(\phi_{e n}, \phi_{e x}, N\right)}{Z \cdot n} \cdot \frac{1}{\tau_{c} s+1}
$$

Donde $K_{c}\left(\mathrm{~N} / \mathrm{mm}^{2}\right), a(\mathrm{~mm})$ y $b\left(\phi_{e n}, \phi_{e x}, Z\right)$ son el coeficiente de fuerza, la profundidad axial de corte y la función de inmersión en el corte respectivamente. La función $b$ no tiene unidades y varía entre 0 y $Z$ en función de los ángulos de entrada y salida y del número de dientes implicados en el corte. Tanto la profundidad axial de corte como la función de inmersión son parámetros variables a lo largo del recorrido de la herramienta y dependen de la geometría de la pieza.

Si apareciese el fenómeno del retemblado el proceso se volvería inestable produciendo oscilaciones de gran amplitud en las fuerzas de corte. En ese caso el proceso se asimilaría a un sistema no lineal de alto orden y no podría ser controlado mediante el avance con un control adaptativo. El control de procesos con retemblado ha de tratarse, por tanto, separadamente y, por tanto, se deberá desactivar el control adaptativo cuando aparezca retemblado en el proceso.

Aunque la dinámica de la máquina no varía con el tiempo es más práctico considerar el conjunto máquina-proceso como variable. De esta manera, la función de transferencia del conjunto puede expresarse de la siguiente manera:

$$
G_{c}(s)=\frac{1}{\tau_{m} s+1} \cdot \frac{K_{c} \cdot a \cdot b}{N \cdot n \cdot\left(\tau_{c} s+1\right)}=\frac{K_{p}}{\left(\tau_{m} s+1\right) \cdot\left(\tau_{c} s+1\right)}
$$

Donde $K_{p}(\mathrm{~N} / \mathrm{mm} / \mathrm{s})$ es la ganancia del proceso y responde a la expresión:

$$
K_{p}=\frac{K_{c} \cdot a \cdot b}{Z \cdot n}
$$

En realidad $\tau_{c}$ puede variar debido a las deflexiones del conjunto herramienta-pieza, que a su vez afectarían al espesor de viruta. Sin embargo, este parámetro se considerará constante en una primera aproximación al estudio de un algoritmo de control adaptativo. Dado que el proceso se controla mediante un periodo de muestreo $T$, la función de transferencia en $z$ equivalente será: 


$$
G_{c}(z)=\frac{F_{p}(k)}{f_{c}(k)}=\left(1-z^{-1}\right) \cdot z \frac{G_{c}(s)}{s}=\frac{b_{0} z+b_{1}}{z^{2}+a_{1} z+a_{2}}
$$

Donde $k$ es el índice de vueltas del husillo, $z$ el operador de adelanto y los parámetros se pueden expresar de la siguiente manera:

$$
\begin{aligned}
& b_{0}=K_{p} \cdot \frac{\tau_{m}\left(1-e^{-T / \tau_{m}}\right)-\tau_{c}\left(1-e^{-T / \tau_{c}}\right)}{\tau_{m}-\tau_{c}} \\
& b_{1}=K_{p} \cdot \frac{\tau_{c}\left(1-e^{-T / \tau_{c}}\right) e^{-T / \tau_{m}}-\tau_{m}\left(1-e^{-T / \tau_{m}}\right) e^{-T / \tau_{c}}}{\tau_{m}-\tau_{c}} \\
& a_{1}=-\left(e^{-T / \tau_{m}}+e^{-T / \tau_{c}}\right) \\
& a_{2}=e^{-T\left(1 / \tau_{c}+1 / \tau_{m}\right)}
\end{aligned}
$$




\section{REFERENCIAS BIBLIOGRÁFICAS}

[1] E. Brousseau, S. Dimov, D. Pham, Some recent advances in multi-material microand nano-manufacturing, The International Journal of Advanced Manufacturing Technology. 47 (2010) 161-180.

[2] N. Taniguchi, Current Status in, and Future Trends of, Ultraprecision Machining and Ultrafine Materials Processing, CIRP Ann. Manuf. Technol. 32 (1983) 573-582.

[3] T. Masaki, K. Kawata, T. Masuzawa, Micro electro-discharge machining and its applications, Proceedings. IEEE Micro Electro Mechanical Systems-An Investigation of Micro Structures, Sensors, Actuators, Machines. (1990) 21-26.

[4] K. Nakamoto, T. Ishida, N. Kitamura, Y. Takeuchi, Fabrication of microinducer by 5-axis control ultraprecision micromilling, CIRP Ann. Manuf. Technol. In Press, Corrected Proof (2011) 407-410.

[5] C.L. Kuo, T. Masuzawa, M. Fujino, High-precision micronozzle fabrication, (1992) 116-121.

[6] S. Kalpakjian, S.R. Schmid, Manufacturing Engineering and Technology, 5th ed., Pearson. Prentice Hall, 1992.

[7] G. Boothroyd, W. Knight, Fundamental of Machining and Machine Tools Marcel Dekker, 2nd ed., , 1989.

[8] S. Coromat, Mecanizado Moderno: Manual Práctico, Sandvik Coromat, 1994.

[9] T.L. Schmitz, K.S. Smith, Machining Dynamics: Frequency Response to Improved Productivity, Springer Verlag, 2008.

[10] S. Bouzid, N. Ben Salah, J.L. Lebrun, Influence of machining by finishing milling on surface characteristics, Int. J. Mach. Tools Manuf. 41 (2001) 443-450. 
[11] M.P. Groover, Fundamentals of Modern Manufacturing: Materials Processes, and Systems, Wiley-India, 2007.

[12] G. Boothroyd, P. Dewhurst, W. Knight, Product Design for Manufacture and Assembly, New York: Marcel Dekker, Inc, 1994.

[13] L. Esteban, J. María, Tecnología Mecánica y Metrotecnia, Donostiarra, San Sebastián, 1984.

[14] M. Martellotti, An analysis of the milling process, part II-Down milling, Trans ASME. 67 (1945) 233-251.

[15] F. Koenigsberger, A. Sabberwal, An investigation into the cutting force pulsations during milling operations, International Journal of Machine Tool Design and Research. 1 (1961) 15-33.

[16] A. Sabberwal, Chip section and cutting force during the milling operation, Annals of the CIRP. 10 (1961) 197-203.

[17] G. Byrne, D. Dornfeld, B. Denkena, Advancing Cutting Technology, CIRP Ann. Manuf. Technol. 52 (2003) 483-507.

[18] F. Klocke, G. Eisenblätter, Dry Cutting, CIRP Ann. Manuf. Technol. 46 (1997) 519-526.

[19] U. Heisel, A. Feinauer, Dynamic Influence on Workpiece Quality in High Speed Milling, CIRP Ann. Manuf. Technol. 48 (1999) 321-324.

[20] H.K. Tönshoff, I. Inasaki, Sensors Applications, Volume 1, Sensors in Manufacturing, Wiley-VCH, April 2001. 1 (2001).

[21] G. Byrne, D. Dornfeld, I. Inasaki, G. Ketteler, W. König, R. Teti, Tool Condition Monitoring (TCM) - The Status of Research and Industrial Application, CIRP Ann. Manuf. Technol. 44 (1995) 541-567.

[22] H. Tönshoff, H.G. Wobker, M. Hartmann, P. Kilsmark, The future of monitoring, VDI BERICHTE. 1179 (1995) 347-347. 
[23] F. Klocke, M. Reuber, Process monitoring in sculptured surface milling, Production Engineering, Research and Development. 7 (2000) 1-4.

[24] F. Klocke, H. Kratz, Process monitoring in mould and die milling with accelerometer signals, Production Engineering, Research and Development. 8 (2001) $45-48$.

[25] Y. Altintas, Manufacturing Automation: Metal Cutting Mechanics, Machine Tool Vibrations, and CNC Design, Cambridge University Press, 2000.

[26] M.C. Shaw, Metal Cutting Principles, Clarendon press, 1986.

[27] M.E. Merchant, Mechanics of the metal cutting process. I. Orthogonal cutting and a type 2 chip, J. Appl. Phys. 16 (1945) 267-275.

[28] F. Taylor, On the art of metal cutting, Trans.ASME. 28 (1907) 31-350.

[29] G. Yucesan, Y. Altintas, Improved modelling of cutting force coefficients in peripheral milling, Int. J. Mach. Tools Manuf. 34 (1994) 473-487.

[30] E. Armarego, A generic mechanics of cutting approach to predictive technological performance modeling of the wide spectrum of machining operations, Mach. Sci. Technol. 2 (1998) 191-211.

[31] E.J.A. Armarego, H. Zhao, Predictive Force Models for Point-Thinned and Circular Centre Edge Twist Drill Designs, CIRP Ann. Manuf. Technol. 45 (1996) 6570.

[32] J. Kahles, Machinability data requirements for advanced machining systems, CIRP Ann. 36 (1987) 523-529.

[33] W.A. Kline, R.E. DeVor, J.R. Lindberg, The prediction of cutting forces in end milling with application to cornering cuts, International Journal of Machine Tool Design and Research. 22 (1982) 7-22. 
[34] J.W. Sutherland, R.E. DeVor, Improved Method for Cutting Force and Surface Error Prediction in Flexible End Milling Systems, Journal of engineering for industry. 108 (1986) 269-279.

[35] P. Tlusty J and MacNeil, Dynamics of cutting forces in end milling, CIRP Ann. 24 (1975) 21-25.

[36] E.J.A. Armarego, N.P. Deshpande, Computerized End-Milling Force Predictions with Cutting Models Allowing for Eccentricity and Cutter Deflections, CIRP Ann. Manuf. Technol. 40 (1991) 25-29.

[37] R.E. DeVor, W. Kline, W.J. Zdeblick, A mechanistic model for the force system in end milling with application to machining airframe structures, 8th North American Manufacturing Research Conference. 8 (1980) 297-303.

[38] N. Zlatin, M. Field, V.A. Tipnis, R.C. Garrison, J.D. Christopher, Establishment of Production Machinability Data, DTIC Document, 1975.

[39] W.A. Kline, R.E. DeVor, The effect of runout on cutting geometry and forces in end milling, International Journal of Machine Tool Design and Research. 23 (1983) 123-140.

[40] H.J. Fu, R. DeVor, S. Kapoor, A mechanistic model for the prediction of the force system in face milling operations, Journal of engineering for industry. 106 (1984).

[41] E. Budak, Y. Altintas, E.J.A. Armarego, Prediction of milling force coefficients from orthogonal cutting data, J. Manuf. Sci. Eng. Trans. ASME. 118 (1996) 216-224.

[42] S. Smith, J. Tlusty, An overview of modeling and simulation of the milling process, Journal of engineering for industry. 113 (1991) 169-175.

[43] General Electric Company, Milling Handbook of High-Efficiency Metal Cutting, Carboloy Systems Department, 1980.

[44] J. Tlusty, Machine Dynamics. Handbook of High-Speed Machining Technology, R. I. King, ed., Chapman and Hall, New York, 1985. 
[45] W. Kline, R. DeVor, I. Shareef, The prediction of surface accuracy in end milling, ASME Journal of Engineering for Industry. 104 (1982) 272-278.

[46] T. Babin, J. Lee, J. Sutherland, S. Kapoor, A model for end milled surface topography, (1985) 362-368.

[47] J. Tlusty, F. Ismail, Special aspects of chatter in milling, Trans.ASME. 105 (1983) p24.

[48] J. Sutherland, R. DeVor, A Dynamic Model for the Cutting Force System in the End Milling Process, Sensors and Controls for Manufacturing. 33 (1988) 53-62.

[49] S.G. Kapoor, R.E. DeVor, R. Zhu, R. Gajjela, G. Parakkal, D. Smithey, Development of mechanistic models for the prediction of machining performance: Model building methodology, Mach. Sci. Technol. 2 (1998) 213-238.

[50] E.J.A. Armarego, J. Wang, N.P. Deshpande, Computer-Aided Predictive Cutting Model for Forces in Face Milling Allowing for Tooth Run-Out, CIRP Ann. Manuf. Technol. 44 (1995) 43-48.

[51] Y. Altintaş, P. Lee, A General Mechanics and Dynamics Model for Helical End Mills, CIRP Ann. Manuf. Technol. 45 (1996) 59-64.

[52] D. Dornfeld, S. Min, Y. Takeuchi, Recent Advances in Mechanical Micromachining, CIRP Ann. Manuf. Technol. 55 (2006) 745-768.

[53] M. Movahhedy, M.S. Gadala, Y. Altintas, Simulation of the orthogonal metal cutting process using an arbitrary Lagrangian-Eulerian finite-element method, J. Mater. Process. Technol. 103 (2000) 267-275.

[54] E. Ceretti, P. Fallböhmer, W. Wu, T. Altan, Application of 2D FEM to chip formation in orthogonal cutting, J. Mater. Process. Technol. 59 (1996) 169-180.

[55] T. Özel, E. Zeren, Finite element modeling of stresses induced by high speed machining with round edge cutting tools, (2005). 
[56] P. Rai-Choudhury, Handbook of Microlithography, Micromachining, and Microfabrication, Inspec/Iee, 1997.

[57] M. Takács, B. Verö, I. Mészáros, Micromilling of metallic materials, J. Mater. Process. Technol. 138 (2003) 152-155.

[58] C. Friedrich, P. Coane, J. Goettert, N. Gopinathin, Direct fabrication of deep x-ray lithography masks by micromechanical milling, Precis Eng. 22 (1998) 164-173.

[59] C.R. Friedrich, M.J. Vasile, Development of the micromilling process for highaspect-ratio microstructures, Microelectromechanical Systems, Journal of. 5 (1996) 3338.

[60] C. Friedrich, P. Coane, M. Vasile, Micromilling development and applications for microfabrication, Microelectronic engineering. 35 (1997) 367-372.

[61] H. Weule, V. Hüntrup, H. Tritschler, Micro-Cutting of Steel to Meet New Requirements in Miniaturization, CIRP Ann. Manuf. Technol. 50 (2001) 61-64.

[62] D. Spath, V. Hüntrup, Micro-milling of Steel for Mold Manufacturing - Influences of Material, Tools and Process parameters, 1st International EUSPEN conference proceedings. 1 (1999) 203-206.

[63] S. Shimada, N. Ikawa, H. Tanaka, J. Uchikoshi, Structure of Micromachined Surface Simulated by Molecular Dynamics Analysis, CIRP Ann. Manuf. Technol. 43 (1994) 51-54.

[64] G. Bissacco, H.N. Hansen, J. Slunsky, Modelling the cutting edge radius size effect for force prediction in micro milling, CIRP Ann. Manuf. Technol. 57 (2008) 113-116.

[65] W.Y. Bao, I.N. Tansel, Modeling micro-end-milling operations. Part I: analytical cutting force model, Int. J. Mach. Tools Manuf. 40 (2000) 2155-2173.

[66] P.K. Basuray, B.K. Misra, G.K. Lal, Transition from ploughing to cutting during machining with blunt tools, Wear. 43 (1977) 341-349. 
[67] M. Malekian, S. Park, Investigation of micro milling forces for aluminum, Transactions of SME-NAMRI. 35 (2007) 417-424.

[68] W.Y. Bao, I.N. Tansel, Modeling micro-end-milling operations. Part II: tool runout, Int. J. Mach. Tools Manuf. 40 (2000) 2175-2192.

[69] W.Y. Bao, I.N. Tansel, Modeling micro-end-milling operations. Part III: influence of tool wear, Int. J. Mach. Tools Manuf. 40 (2000) 2193-2211.

[70] T. Özel , X. Liu, A. Dhanorker, Modelling and Simulation of Micro-Milling Process, 4th International Conference and Exhibition on Design and Production of Machines and Dies/Molds. (2007).

[71] H. Pérez, A. Vizán, J.C. Hernandez, M. Guzmán, Estimation of cutting forces in micromilling through the determination of specific cutting pressures, J. Mater. Process. Technol. 190 (2007) 18-22.

[72] G. Newby, S. Venkatachalam, S.Y. Liang, Empirical analysis of cutting force constants in micro-end-milling operations, J. Mater. Process. Technol. 192-193 (2007) 41-47.

[73] Y. Altintas, D. Montgomery, Mechanism of cutting force and surface generation in dynamic milling, ASME Journal of Engineering for Industry. 113 (1991) 160-168.

[74] G.F. Micheletti, T.L. Doménech, Mecanizado Por Arranque De Viruta: Tecnología Mecánica, Blume, 1980.

[75] J. Tlusty, M. Polacek, Beispiele der behandlung der selbsterregten Schwingung der Werkzeugmaschinen FoKoMa, (1957).

[76] S. Tobias, W. Fishwick, Theory of Regenerative Machine Tool Chatter, , 1958.

[77] J. Tlusty, Analysis of the state of research in cutting dynamics, Annals of the CIRP. 27 (1978) 583-589.

[78] E.I. Rivin, Tooling Structure: Interface between Cutting Edge and Machine Tool, CIRP Ann. Manuf. Technol. 49 (2000) 591-643. 
[79] Y. Altintas, M. Weck, Chatter Stability of Metal Cutting and Grinding, CIRP Ann. Manuf. Technol. 53 (2004) 619-642.

[80] Y. Altintaş, E. Budak, Analytical Prediction of Stability Lobes in Milling, CIRP Ann. Manuf. Technol. 44 (1995) 357-362.

[81] J. Tlusty, Manufacturing Processes and Equipment, Prentice Hall, 1999.

[82] R. Kegg, T.R. Sisson, Troubleshooting Chatter by Improving Cutting Conditions, ASME, Technical Publication. MR 68-615 (1968).

[83] R.L. Kegg, Cutting Dynamics in Machine Tool Chatter, Journal of Engineering for Industry. 87 (1965) 464-470.

[84] F. Koenigsberger, J. Tlusty, Machine Tool Structures, Stability Against Chatter, Pergamon Press, New York, 1970.

[85] J. Peters, P. Vanherck, Theory and Practice of Fluid Dampers in Machine Tools, 57 (1969) 69.

[86] S. Smith, T.P. Jacobs, J. Halley, The Effect of Drawbar Force on Metal Removal Rate in Milling, CIRP Ann. Manuf. Technol. 48 (1999) 293-296.

[87] Y. Altintas, C. Brecher, M. Weck, S. Witt, Virtual Machine Tool, CIRP Ann. Manuf. Technol. 54 (2005) 115-138.

[88] O. Maeda, Y. Cao, Y. Altintas, Expert spindle design system, Int. J. Mach. Tools Manuf. 45 (2005) 537-548.

[89] F. Rodríguez, M. López, Control adaptativo y robusto, Universidad de Sevilla, España. (1996).

[90] Y. Altintas, F. Sassani, F. Ordubadi, Design and analysis of adaptive controllers for milling process, CSME. 14 (1990) 17-25.

[91] F. Rasmussen, Principal developments in the adaptive control of machine tools, Journal of Dynamic Systems, Measurement, and Control. 105 (1983) 107. 
[92] B. Beadle, J. Bollinger, Computer adaptive control of a machine tool, Annals of the CIRP. 16 (1971) 61-65.

[93] J. Tlusty, M. Elbestawi, Analysis of Transient in an Adaptive Control Servomechanism for Milling with Constant Force, Journal of engineering for industry. 99 (1977).

[94] I. Tansel, O. Rodriguez, M. Trujillo, E. Paz, W. Li, Micro-end-milling-I. Wear and breakage, Int. J. Mach. Tools Manuf. 38 (1998) 1419-1436.

[95] J. Tlusty, M. Elbestawi, Constraints in adaptive control with flexible end mills, Annals of the CIRP. 28 (1979) 253-255.

[96] I. Tansel, A. Nedbouyan, M. Trujillo, B. Tansel, Micro-end-milling-II. Extending tool life with a Smart Workpiece Holder (SWH), Int. J. Mach. Tools Manuf. 38 (1998) $1437-1448$.

[97] I.N. Tansel, T.T. Arkan, W.Y. Bao, N. Mahendrakar, B. Shisler, D. Smith, M. McCool, Tool wear estimation in micro-machining.: Part I: tool usage-cutting force relationship, Int. J. Mach. Tools Manuf. 40 (2000) 599-608.

[98] I. Tansel, M. Trujillo, A. Nedbouyan, C. Velez, W. Bao, T.T. Arkan, B. Tansel, Micro-end-milling-III. Wear estimation and tool breakage detection using acoustic emission signals, Int. J. Mach. Tools Manuf. 38 (1998) 1449-1466.

[99] S. Das, A. Chattopadhyay, A. Murthy, Force parameters for on-line tool wear estimation: a neural network approach, Neural Networks. 9 (1996) 1639-1645.

[100] H. Takeshita, I. Inasaki, Monitoring of milling process with an acoustic emission sensor, Jap. Soc. Precision Eng. 59 (1993) 269-269.

[101] I. Nur Tansel, Identification of the prefailure phase in microdrilling operations using multiple sensors, Int. J. Mach. Tools Manuf. 34 (1994) 351-364.

[102] M.T. Zaman, A.S. Kumar, M. Rahman, S. Sreeram, A three-dimensional analytical cutting force model for micro end milling operation, Int. J. Mach. Tools Manuf. 46 (2006) 353-366. 
[103] M. Wan, W. Zhang, Systematic study on cutting force modelling methods for peripheral milling, Int. J. Mach. Tools Manuf. 49 (2009) 424-432.

[104] H. Zheng, X. Li, Y. Wong, A. Nee, Theoretical modelling and simulation of cutting forces in face milling with cutter runout, Int. J. Mach. Tools Manuf. 39 (1999) 2003-2018.

[105] E.J.A. Armarego, N.P. Deshpande, Computerized Predictive Cutting Models for Forces in End-Milling Including Eccentricity Effects, CIRP Ann. Manuf. Technol. 38 (1989) 45-49.

[106] P. Rodriguez, H. Perez, J. Labarga, A. Vizan, Research on Tool Life in MicroEnd-Milling as reltaed to workpiece quality criteria, Proceedings of the ETC. (2008).

[107] W.A. Nash, Schaum's Outline of Theory and Problems of Strength of Materials, Schaum's Outline Series, 1998.

[108] C. Li, X. Lai, H. Li, J. Ni, Modeling of three-dimensional cutting forces in microend-milling, J Micromech Microengineering. 17 (2007) 671-678.

[109] R.E. Kalman, R.S. Bucy, New results in linear filtering and prediction theory, Journal of Basic Engineering. 83 (1961) 95.

[110] J. Lindqvist-Gauffin, Inverse filtering. Instrumentation and techniques, Speech Transmission Laboratory Quarterly Progress and Status Report. 5 (1964) 1-4. 